\title{
Service Oriented Approach to \\ High Performance Scientific Computing
}

\author{
Jaison Paul Mulerikkal
}

February 2013

A thesis submitted for the degree of Doctor of Philosophy of the Australian National University

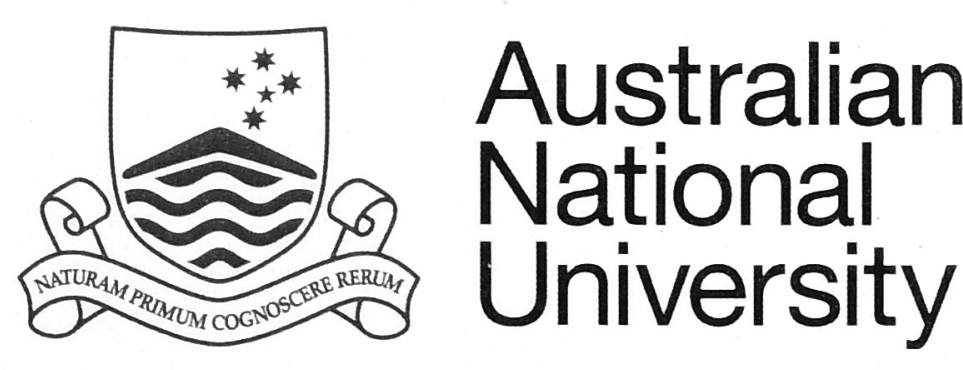



Dedicated to Lalson Paul Mulerikkal, my late beloved brother and to those who struggle to reconcile reason with the Divine 


\section{Declaration}

The work in this thesis is my own except where otherwise stated.

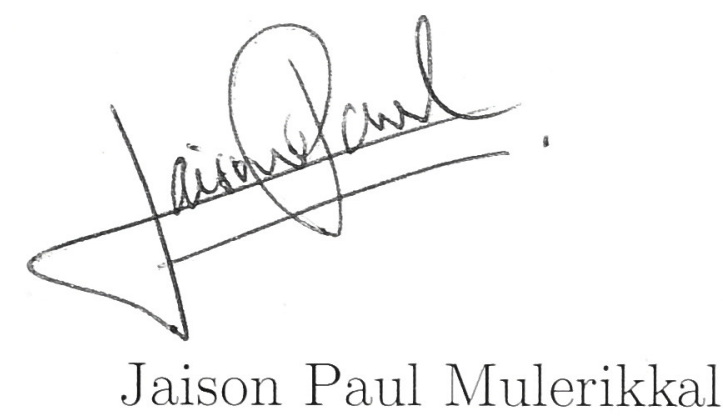




\section{Acknowledgements}

Heartfelt thanks to Dr Peter Strazdins for his proactive and persistent guidance throughout the project as the chief supervisor and gratefully acknowledge the contributions of Dr Henry Gardner and Dr Alistair Rendell as members of the supervisory panel.

Special thanks to my colleagues and friends - Danny Robson, Muhammad Atif, Jia Cai, Josh Milthrope and Teena Thaliath for their support and warm friendship that helped me to complete this work. I am also happy to acknowledge the valuable assistance provided by Boby Jose Thekkanath in cloud enabling the project and Peter Sharman for his diligent proof reading efforts.

I thank Australian Research Council (ARC) for its support to this PhD research through Linkage Grant LP0669762. I would also like to thank Platform Computing (Now IBM-Platform) for their industry partnership with this project through a formal agreement with ANU. 


\section{Related Publications}

- J. Mulerikkal, P. Strazdins, B. J. Thekkanath, Enabling SOA approach for High Performance Cloud Computing using an Efficient Data Service, in Proceedings of IEEE Asia Pacific Cloud Computing Congress, Shenzhen, China: IEEE, November 2012.

- J. Mulerikkal and P. Strazdins, Efficient data service design for a soa approach to scientific computing, in Proceedings of the Tenth IASTED International Conference on Parallel and Distributed Computing and Networks (PDCN 2011), Innsbruck: ACTA Press, February 2011, pp. 46-53.

- J. Mulerikkal and P. Strazdins, An SOA approach to high performance scientific computing - early experiences, in Proceedings of IEEE International Conference on High Performance Computing 2010 (HiPC 2010), Goa, India: IEEE, December 2010, pp. 1-10.

- J. Mulerikkal and P. Strazdins, Service oriented approach to high performance scientific computing, in Proceedings of the Seventh IEEE International Symposium on Cluster Computing and the Grid (CCGRID 2010), Melbourne: IEEE, June 2010, pp. 820-825.

- The ANU-SOAM is released as open-source product under BSD licence. It is available at: http://cs.anu.edu.au/ Jaison. Mulerikkal 


\section{Abstract}

Service Oriented Architectures (SOA) are used in business circles to harness the distributed resources of an enterprise, with relative ease and convenience. SOA is perceived to deliver programmability, scalability and efficiency under heterogeneity. The scientific community is always aspired to have a similar convenient approach to solve "not so embarrassingly parallel" scientific problems with expected levels of high performance, especially in heterogeneous conditions. One of the major challenges in parallelizing scientific algorithms is their apparent interdependency of tasks (atomic units of parallel works) which results in too fine granularity. The computational advantages in parallelizing those scientific algorithms can be overshadowed by costs involved in communications between non-optimal fine-grained tasks, in a SOA environment.

The aim of this $\mathrm{PhD}$ research is to overcome these challenges and to empower scientists and researchers with SOA tools to develop high performance scientific applications with relative ease that can perform well under heterogeneous environments. The research has produced a scalable and heterogeneity-oblivious SOA middleware - ANU-SOAM. It implements a popular enterprise SOA middleware API (IBM-Platform Symphony API) and thus ensures programmability. It offers better performance under heterogeneous conditions by implementing load balancing and scheduling techniques. Along with its compute services it provides a Data Service which helps application programmers to develop codes that can effectively circumvent the interdependency of tasks and thereby reduce communications to ensure high performance outcomes. The Data Service achieves this by allowing data to be stored, accessed, modified and synchronized (using add, get, put and sync functionalities) at host and compute nodes according to the application logic. It is also observed that the programming model supported by the Data Service can help ANU-SOAM applications to access compute resources in a "Cloud IaaS" over high latency networks (like the Internet) with much lower overheads compared to the conventional SOA programming models. 



\section{Contents}

Acknowledgements vii

Related Publications $\quad$ ix

Abstract $\quad$ xi

Notation xix

1 Introduction 1

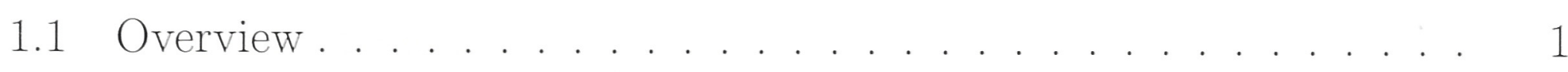

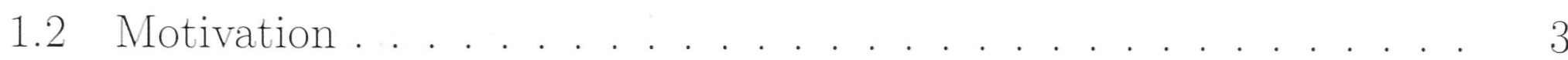

1.2.1 Convenience of SOA Approach to Scientific Application Programmers ........................... 3

1.2.2 High Performance Capabilities to Not so Embarrassingly Parallel Scientific Problems . . . . . . . . . . . . . 4

1.2.3 Efficiency for Scientific Applications on Heterogeneous and Cloud Environments . . . . . . . . . . . . . . 4

1.3 Scope of the Thesis . . . . . . . . . . . . . . . . . 4

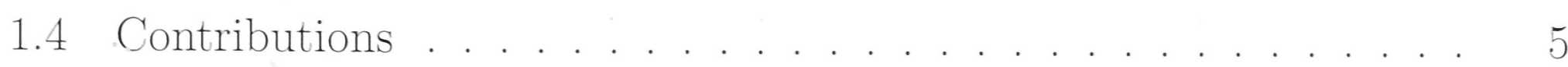

1.5 Thesis Organization . . . . . . . . . . . . . . . . . 6

2 Background 7

2.1 Classifications of Scientific Problems . . . . . . . . . . . 7

2.1.1 Coarse-grained Parallel Problems . . . . . . . . . . . . 8

2.1.2 Medium-grained Parallel Problems . . . . . . . . . . . . 9

2.1.3 Fine-grained Parallel Problems . . . . . . . . . . . . . 10

2.2 Different Approaches to HPSC . . . . . . . . . . . . . . . . . 10

2.2.1 Service Oriented Architecture ... . . . . . . . . . 10

2.2 .2 Grid Approach . . . . . . . . . . . . . . 16 
2.2.3 Other Approaches . . . . . . . . . . . . . . . . . . 19

2.3 Convergence in Cloud Computing . . . . . . . . . . . . . . . . 20

2.3.1 Definitions and Classifications ... . . . . . . . . 22

2.3.2 Cloud Computing Software Platforms . . . . . . . . . . . . 24

2.3.3 Grid and Cloud Computing . . . . . . . . . . 25

2.3.4 SOA and Cloud Computing . . . . . . . . . . 25

2.3.5 Cloud Computing for HPSC . . . . . . . . . . . . 26

2.4 Case Study: Platform Symphony . . . . . . . . . . . . . . . . . 27

2.4.1 Architecture . . . . . . . . . . . . . . . 28

2.4.2 Strengths and Challenges. . . . . . . . . . . . 29

2.5 Chapter Conclusions . . . . . . . . . . . . . . . . . 30

3 Related Work 31

3.1 Message Passing Systems . . . . . . . . . . . . . . . . . . . 31

3.1.1 Message Passing Interface . . . . . . . . . . . . . . . . 31

3.1.2 EasyGrid . . . . . . . . . . . . . . . . 32

3.1.3 HeteroMPI . . . . . . . . . . . . . . . . 33

3.1 .4 MPI PCM Library . . . . . . . . . . . . . . . . . 34

3.2 Shared Memory Paradigms . . . . . . . . . . . . . . . . 35

3.2 .1 OpenMP . . . . . . . . . . . . . . . . . . 35

3.2 .2 Global Arrays . . . . . . . . . . . . . . . . . . 36

3.3 Distributed File System Services . . . . . . . . . . . . . . . . . . 37

3.3.1 Google File System . . . . . . . . . . . . . . . . . 38

3.3.2 Hadoop Distributed File System . . . . . . . . . . . . 38

3.4 Chapter Conclusions . . . . . . . . . . . . . . . 38

4 Design and Implementation 41

4.1 Architecture . . . . . . . . . . . . . . . . . 41

4.1.1 Design Requirements . . . . . . . . . . . . . . . 42

4.1.2 Client Module . . . . . . . . . . . . . . . . . 43

4.1 .3 Service Module . . . . . . . . . . . . . . . . . . 44

4.1.4 Resource Manager Module . . . . . . . . . . . . . . . . . . 44

4.1.5 Messages, Tasks and Tasklets . . . . . . . . . . . . 46

4.1.6 Data Service Extension . . . . . . . . . . . . . . . . . . . 46

4.2 Application Development . . . . . . . . . . . . . . . 53

4.2.1 Conventional SOA Approach . . . . . . . . . . . 53

4.2.2 ANU-SOAM Approach . . . . . . . . . . . . 57 
4.3 Application Deployment . . . . . . . . . . . . . . . . . . . 59

4.4 ANU-SOAM on Cloud . . . . . . . . . . . . . . . . . . 61

4.4.1 A PaaS for Scientific Computing . . . . . . . . . . . 61

4.4.2 Enable Real time Applications on Cloud . . . . . . . . . . 61

4.4.3 A Testbed for IBM-Platform Symphony Compatible Cloud Application Development and Integration . . . . . . . . 63

4.5 Chapter Conclusions ... . . . . . . . . . . . 63

5 Programming Examples $\quad 65$

5.1 N Body Solver . . . . . . . . . . . . . . . . . . 65

5.1.1 Algorithm . . . . . . . . . . . . . . . 65

5.1 .2 Development Features . . . . . . . . . . . . . . . 66

5.1.3 Application Programming . . . . . . . . . . . . . 67

5.2 Heat Transfer Equation Solver . . . . . . . . . . . . . . . . . . 68

5.2.1 Algorithm . . . . . . . . . . . . . . . 68

5.2.2 Development Features . . . . . . . . . . . . . . . . 71

5.2.3 Application Programming . . . . . . . . . . . . . . 71

5.3 Conjugate Gradient . . . . . . . . . . . . . . . . . . . 73

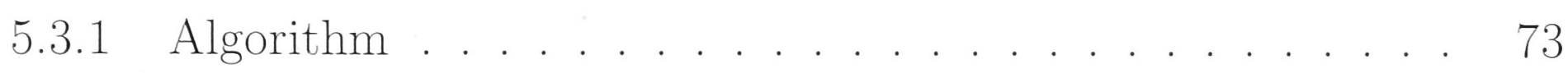

5.3 .2 Development Features . . . . . . . . . . . . . 74

5.3.3 Application Programming . . . . . . . . . . . . . . 77

5.4 Cholesky Factorization . . . . . . . . . . . . . . . . . . . 78

5.4.1 Algorithm . . . . . . . . . . . . . . . . . 78

5.4 .2 Development Features . . . . . . . . . . . . . . . 78

5.4.3 Application Programming . . . . . . . . . . . . . . 80

5.5 Chapter Conclusions . . . . . . . . . . . . . . . . 81

6 Evaluation $\quad 83$

6.1 Homogeneous Environment . . . . . . . . . . . . . . . . . . . . 84

6.1.1 Homogeneous Experimental Environment. . . . . . . . . . 84

6.1.2 Performance and Speedup . . . . . . . . . . . . . . 84

6.1.3 Performance Comparison Against Conventional SOA Programming Model . . . . . . . . . . . . . . . . 92

6.1.4 Performance Comparison Against a Native MPI Application 95

6.1.5 Effects of Varying Task Numbers . . . . . . . . . . . . . . 97

6.2 Heterogeneous Environment . . . . . . . . . . . . . . . . . . . 99

6.2.1 Heterogeneous Experimental Environment . . . . . . . . . 99 
6.2.2 Performance Analysis of Load Balancing Efforts . . . . . . 100

6.3 Cloud Environment . . . . . . . . . . . . . . . . . . 107

6.3.1 Cloud Experimental Environment . . . . . . . . . . . . 107

6.3.2 Performance within Cloud . . . . . . . . . . . . . . 110

6.3.3 Performance by Accessing Cloud over the Internet . . . . . 110

6.4 Chapter Conclusions . . . . . . . . . . . . . . . 112

7 Conclusions \& Future Work 115

7.1 Conclusions . . . . . . . . . . . . . . . . . . 115

7.1.1 High Performance SOA for Scientific Computing . . . . . . 115

7.1.2 Efficiency under Heterogeneous Conditions . . . . . . . . . 116

7.1.3 Cloud Compatibility . . . . . . . . . . . . . . 117

7.1.4 Programmability for Scientific Application Development . 117

7.2 Future Work . . . . . . . . . . . . . . . . . . . . . 117

7.2.1 Fault Tolerance . . . . . . . . . . . . . . . . . . 117

7.2.2 Dynamic Load balancing . . . . . . . . . . . . . . . . . . 118

7.2.3 Support for Simultaneous Multiple Applications . . . . . . 118

\section{Bibliography}

A API

A.1 Client API . . . . . . . . . . . . . . . . . . . . . 135

A.1.1 SoamFactory Class Reference . . . . . . . . . . . 135

A.1.2 SessionCreationAttributes Class Reference . . . . . . . . 135

A.1.3 ConnectionPtr Class Reference . . . . . . . . . . 136

A.1.4 SessionPtr Class Reference . . . . . . . . . . . . 136

A.1.5 TaskSubmissionAttributes Class Reference . . . . . . . . 138

A.1.6 TaskInputHandlePtr Class Reference . . . . . . . . . . 138

A.1.7 TaskOutputHandlePtr Class Reference . . . . . . . . . 138

A.1.8 EnumItemsPtr Class Reference . . . . . . . . . . . . 139

A.2 Service API . . . . . . . . . . . . . . . . . . . . . . . . . 139

A.2.1 ServiceContainer Class Reference . . . . . . . . . . . 139

A.2.2 SessionContextPtr Class Reference . . . . . . . . . . . 140

A.2.3 Task Class Reference . . . . . . . . . . . . . . . 143

A.3 Message API . . . . . . . . . . . . . . . . . . . . . 143

A.3.1 Message Class Reference . . . . . . . . . . . . . 143

A.3.2 InputStreamPtr Class Reference . . . . . . . . . . . . 143

A.3.3 OutputStreamPtr Class Reference. . . . . . . . . . . 144 
B Code Snippets 145

B.1 Client Module . . . . . . . . . . . . . . . . . . . 145

B.1.1 Session Creation . . . . . . . . . . . . . . . 145

B.1.2 Message Creation . . . . . . . . . . . . . . . . 146

B.1.3 Task Communication . . . . . . . . . . . . . . . . 147

B.2 Service Module . . . . . . . . . . . . . . . . . . . . . 148

B.3 Data Service Module . . . . . . . . . . . . . . . . . 148

$\begin{array}{ll}\text { C Algorithms } & 151\end{array}$ 


\section{Notation}

\begin{tabular}{|c|c|}
\hline $\mathrm{SOA}$ & Service Oriented Architecture \\
\hline $\mathrm{HPC}$ & High Performance Computing \\
\hline HPSC & High Performance Scientific Computing \\
\hline ANU-SOAM & ANU Service Oriented Architecture Middleware \\
\hline API & Application Programming Interface \\
\hline $\mathrm{SDK}$ & Software Development Kit \\
\hline SI & Service Instance \\
\hline $\mathrm{RM}$ & Resource Manager \\
\hline NBS & N Body (Problem) Solver \\
\hline HTS & Heat Transfer (Equation) Solver \\
\hline CGS & Conjugate Gradient Solver \\
\hline CFS & (Left Looking) Cholesky Factorization Solver \\
\hline MPI & Message Passing Interface \\
\hline IaaS & Infrastructure as a Service \\
\hline Paas & Platform as a Service \\
\hline Saas & Software as a Service \\
\hline
\end{tabular}





\section{List of Figures}

1.1 History of Computing: Hegelian Cycles . . . . . . . . . . . . . 2

2.1 The SOA Model . . . . . . . . . . . . . . . . . . . . 11

2.2 SOA Main Concepts . . . . . . . . . . . . . . . . . . . . 13

2.3 Cloud Computing Stack: Based on Service Model . . . . . . . . . 23

2.4 Platform Symphony Architecture . . . . . . . . . . . . . . . 28

4.1 ANU-SOAM Architecture . . . . . . . . . . . . . . . . . 42

4.2 Class Diagram: Client Module . . . . . . . . . . . . . . . . . 43

4.3 Class Diagram: Service Module . . . . . . . . . . . . . . . . . . 44

4.4 Load Balancing Using 'weighted round-robin' . . . . . . . . . . . 45

4.5 Deferred put Action in a SI (for Data Consistency) . . . . . . . . 49

4.6 Common Data 2D Indexing . . . . . . . . . . . . . . . . 52

4.7 Sequence Diagram: Conventional SOA Approach . . . . . . . . . 54

4.8 Sequence Diagram: Advanced ANU-SOAM Approach . . . . . . . 58

4.9 ANU-SOAM Deployment . . . . . . . . . . . . . . . . . 60

4.10. ANU-SOAM in the Cloud Computing Stack . . . . . . . . . . 62

5.1 NBS Sample Simulation . . . . . . . . . . . . . . . 67

5.2 HTS - Grid Breakup . . . . . . . . . . . . . . . . . 69

5.3 HTS Data Grid Indexing - 1 . . . . . . . . . . . . . . 70

5.4 HTS Data Grid Indexing - 2 . . . . . . . . . . . . . . . 70

5.5 CFS Algorithm Explained . . . . . . . . . . . . . . 80

6.1 NBS: Performance and Speedup . . . . . . . . . . . . . . . 85

6.2 HTS: Performance and Speedup . . . . . . . . . . . . . . 86

6.3 CGS - Version 1\&2: Performance and Speedup . . . . . . . . . . 88

6.4 CFS (With Full Matrix Access): Performance . . . . . . . . . . . 90

6.5 CFS (With Partial Matrix Access): Performance and Speedup . . 91 
6.6 ANU-SOAM vs SOA/Symphony Programming Versions: NBS . . 93

6.7 ANU-SOAM vs SOA/Symphony Programming Versions: CGS . . 94

6.8 ANU-SOAM vs MPI Native (Gropp) Application: NBS . . . . . . 96

6.9 MPI (Gropp) NBS: IPM Profile . . . . . . . . . . . . . . . . . . . 96

6.10 NBS and CGS - Varying the Number of Tasks . . . . . . . . . . . 97

6.11 HTS - Varying the Number of Tasks . . . . . . . . . . . . . . . . 98

6.12 ANU CSIT Cluster: Heterogeneous Environment . . . . . . . . . 99

6.13 ANU CSIT Cluster: Relative Performance of Machines . . . . . . 100

6.14 NBS on Heterogeneous Environment . . . . . . . . . . . . . . . 101

6.15 NBS Scaling on Heterogeneous Environment . . . . . . . . . . . . 101

6.16 NBS on Heterogeneous Environment: Performance Comparison . 102

6.17 HTS on Heterogeneous Environment . . . . . . . . . . . . . . . 103

6.18 HTS Scaling on Heterogeneous Environment . . . . . . . . . . . . 104

6.19 CGS on Heterogeneous Environment . . . . . . . . . . . . . . . 105

6.20 CGS Scaling on Heterogeneous Environment . . . . . . . . . . . 105

6.21 CFS on Heterogeneous Enviornment . . . . . . . . . . . . . 106

6.22 ANU-SOAM: Cloud over the Internet . . . . . . . . . . . . . . . . 108

6.23 NBS SOAM \& Symphony Versions within the Rackspace Cloud . 109

6.24 NBS SOAM Version within Cloud \& over the Internet . . . . . . . 110

6.25 NBS Symphony Version within Cloud \& over the Internet . . . . . 111

6.26 NBS SOAM \& Symphony Versions over the Internet . . . . . . . . 112 


\section{Chapter 1}

\section{Introduction}

\subsection{Overview}

German philosopher Georg Wilhelm Friedrich Hegel explained philosophy of history as a dialectic between thesis, anti-thesis and resulting synthesis [1]. Hegal suggests that in history, there could emerge a theory first and it could be confronted by an opposing theory. The dialectic between these opposing theories may find a compromise or consensus by assimilating the main aspects of both, in the due course of time. If we look carefully at the history of computing, especially High Performance Computing (HPC), we could see these Hegelian cycles of thesis and anti-thesis resulting in a synthesis.

It was in 1936 at Cambridge University that Alan Turing conceived the principle of the modern computers and Konrad Zuse, in Berlin, built his first electromachanical computer - Z1 [2]. By the beginning of 1970s Seymour Cray - the father of super-computing - has translated Turing's theoretical device into high performance monolithic machines like the Cray $1 \& 2$ [3]. That was the first thesis - high speed computing with monolithic machines. Later on, with the arrival of cheap commodity computing hardware, the theoretical possibility of achieving the same or comparable speeds by harnessing the distributed computing capabilities of commodity hardware was explored with great success. That was the anti-thesis where the computing paradigm took a turn to promote distributed and parallel hardware architecture [4]. Enterprises as well as research circles embraced this new anti-thesis by setting up on-premise cluster computing facilities using relatively cheaper commodity hardware.

Later developments mostly happened in software architecture, built on distributed and parallel hardware architecture as its base. Many research organiza- 


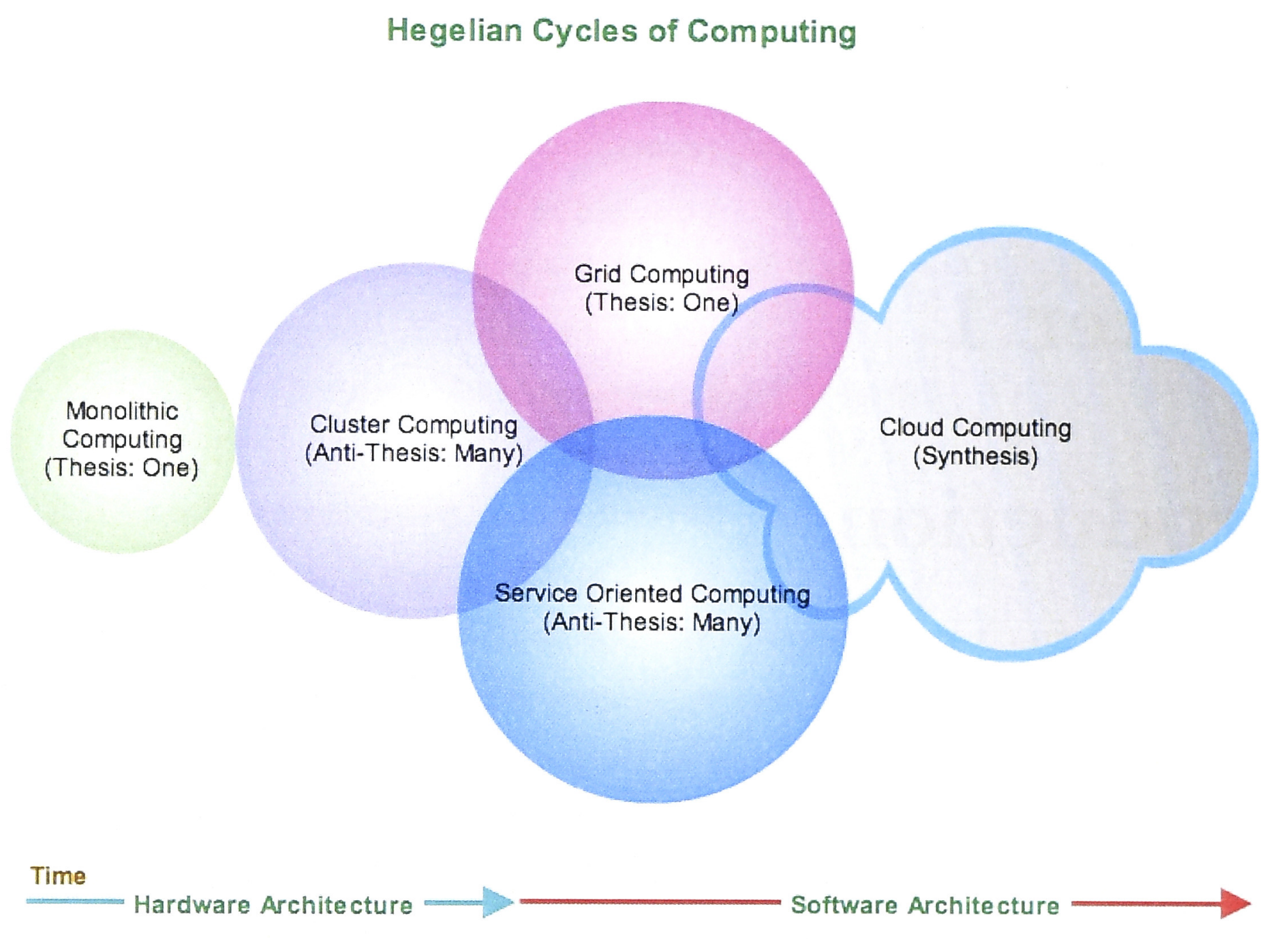

Figure 1.1: History of Computing: Hegelian Cycles

tions with common or similar interests naturally thought of sharing their cluster resources to achieve common goals. This new thesis in software architecture resulted in orchestrating logistically distributed private clusters together to give a logical monolithic appearance to their customers. Grid computing was a result of that new thesis, especially in academic and research circles. Because of the differences in business models and goals, the grid architecture was not a natural choice for enterprises. However to optimize the under-utilized computing resources within an organization a new software approach emerged among enterprise circles, called - Service Oriented Architecture (SOA) (in some cases it was called desktop-grid). The SOA model lies in breaking up a big chunk of otherwise serial computation into smaller atomic units called services that can be parallelized over commodity hardware computational nodes. SOA shared close links to grid computing and vice versa. SOA promoted a distributed software approach that was based on the idea of assembling application components into an interplay between services[5] which were generally operating system or language agnostic. It was an anti-thesis to grid computing because it moved away from the software architecture proposed by the academics to suggest a more fluid architecture. 
From these Hegelian cycles of thesis and anti-thesis in hardware and software designs, recently emerged a new synthesis - cloud computing, where SOA and grid - for that matter enterprise and research communities - coming together around distributed as well as orchestrated hardware and software platforms (refer to Figure 1.1). The most powerful feature of cloud computing is its perceived ability to transfer computing as a 5th utility after water, electricity, gas, and telephony.

High performance scientific computing problems were mainly seeking solutions within grid computing, in recent times. However the convenience and effectiveness of SOA has always eluded scientists and researchers to try solutions within the SOA model. There are still no credible solutions, especially to deal with "not so embarrassingly parallel" scientific problems within SOA environment. There is a genuine need to reconcile this scenario not only to propose a SOA solution to these scientific problems but also to seek new solutions within the cloud computing paradigm which is a synthesis of SOA, grid and many other technologies. In this context, this thesis presents a vision and a model of a service oriented approach to high performance scientific computing which can fit very well within the emerging cloud computing paradigm.

\subsection{Motivation}

\subsubsection{Convenience of SOA Approach to Scientific Appli- cation Programmers}

Scientists and researchers are not necessarily computer programmers. But, they are always at the receiving end of needing high performance computing resources to solve various scientific problems. HPC computing platforms provided them with high performance computing resources, but with the cost of high complexity in developing (coding) those applications. On the other hand, enterprise SOA middlewares like Platform Symphony have worked well for financial applications and its general architecture and API are very well accepted even by amateur programmers [6]. Moreover, the innate fault-tolerance capabilities of SOA model make it desirable for HPSC applications. The scientific community feels the need of a similar SOA infrastructure at their disposal which will be as convenient and effective as enterprise SOA solutions. 


\subsubsection{High Performance Capabilities to Not so Embar- rassingly Parallel Scientific Problems}

The data intensive and "embarrassingly parallel" scientific problems have found credible solutions within SOA and grid systems (refer to section 2.1.1). But, because of the increased granularity from inter-dependency of underlying processes and the resulting overheads, efficient SOA solutions to "not so embarrassingly parallel" scientific problems are very rare. This issue is very well reported in [7] with a block-based square matrix multiplication experiment. This problem can be acute if the consumer (client) tries to access compute services on a 'SOA or cloud' service over a busy or slow network - like the Internet. There is a genuine need for a SOA solution that addresses challenges in "not so embarrassingly parallel" scientific problems with high performance outcomes. (Further discussions are in section 2.1)

\subsubsection{Efficiency for Scientific Applications on Heteroge- neous and Cloud Environments}

Message passing paradigms such as MPI (Message Passing Interface) produce high performance outcomes for many scientific applications. But they are mostly ignorant of the underlying hardware systems and are equipped with very few tools to deal with heterogeneous conditions, even if they are aware of it. SOA is innately equipped to deal with heterogeneous environments [8]. This is because the number of tasks in SOA applications can be greater than the number of compute nodes, which leaves us an opportunity to distribute them among the nodes according to their relative performance (refer to section2.2.1). This property of SOA gives us an opportunity to improve the efficiency of scientific applications in heterogeneous conditions with the help of proper load balancing and scheduling techniques.

\subsection{Scope of the Thesis}

The discussion in section 2.1 briefly explains the wider spectrum of scientific computing. This discussion also sheds light into the fact that even though many grid solutions exist for coarse-grained scientific problems, efficient solutions to "not so embarrassingly parallel" scientific problems are very rare. There is a need to find solutions to deal with "not so embarrassingly parallel" scientific 
problems with relative ease and convenience to the application programmers. So, the scope of this thesis is finely calibrated to address high performance solution to medium-grained scientific problems using SOA architecture. This definition of scope also acknowledges that SOA may not be a viable model to deal with fine-grained parallel problems because of the intrinsic limitations of the SOA model which involves certain level of communication costs associated with it and computational advantages in parallelizing fine-grained tasks cannot overcome this.

\subsection{Contributions}

This thesis presents an efficient high performance SOA paradigm to solve mediumgrained and communication intensive scientific problems using a SOA middleware that can perform well on homogeneous, heterogeneous and cloud environments.

The thesis:

- provides a convenient SOA programming environment för scientific application programmers by developing a service oriented architecture middleware - ANU-SOAM - for high performance scientific computing with convenient APIs (that of Platform Symphony), libraries, configuration files and command line tools.

- addresses the problems arising from finer granularity of "not so embarrassingly parallel" scientific problems in SOA using a Data Service extension to give high performance outcomes.

- improves performance of ANU-SOAM for HPSC under heterogeneous conditions using suitable load balancing and scheduling techniques.

- presents ANU-SOAM solutions for four major scientific kernels and applications - N Body Solver (NBS), Heat Transfer Equation Solver(HTS), Conjugate Gradient Solver (CGS) and Cholesky Factorization Solver (CFS) and evaluates its scalability and performance under homogeneous, heterogeneous and cloud test conditions.

- demonstrates the cloud compatibility of ANU-SOAM and the ability of its programming model to better access cloud resources over Internet compared to conventional SOA models. 


\subsection{Thesis Organization}

The rest of this thesis is organized as follows: Chapter 2 provides necessary background to understand this thesis well and Chapter 3 discusses the related works in this area. Chapter 4 presents the design and the developmental features of ANU-SOAM - the middleware developed as past of this thesis work. Chapter 5 consists of the discussion of 4 diverse scientific kernels/applications - Conjugate Gradient, Cholesky Factorization, N Body Solver and 2D Heat Equation developed using ANU-SOAM API and running on the middleware to evaluate it. Chapter 6 provides a performance evaluation of ANU-SOAM and with some performance comparisons with comparable programming tools, and its scope in the emerging cloud computing systems. The thesis ends with conclusions and suggestions for future work. 


\section{Chapter 2}

\section{Background}

This chapter gives an introduction to the world of scientific kernels and applications that rely on high performance computing to find solutions. It also presents important classifications of those scientific problems to better understand the scope of this thesis. This chapter further gives an account of different approaches and architectures that are used to address these problems in academic as well as in enterprise circles. It also gives an account of how these two different streams found synergy in converging their philosophies to give rise to the cloud computing paradigm.

\subsection{Classifications of Scientific Problems}

HPC scientific problems can generally be classified into data intensive or computation intensive problems. Data intensive problems are those which demand huge data transfer compared to their computational efforts. Examples for data intensive scientific applications are some problems in High Energy Physics (HEP) [9][10][11], where terabytes or petabytes of data produced by tools like Large Hadron Collider has to be analyzed to produce results [11]. Computation intensive applications are the ones that demand large computational effort, even if the input data is very small. Many linear algebraic problems are computational intensive problems. There are also problems like genomics protein structure prediction and structural genomics [12] in bioinformatics, which are both data and computationally intensive.

One of the other important classification of scientific problems in HPC - which is more relevant to this thesis - is based on the concept of granularity. The modern HPC technique is to divide huge processing blocks of computation into 
smaller ones to parallelize them. In parallel computing, periods of computation are separated by periods of communication. Here granularity means a measure of the ratio of computation to communication. Based on the granularity of those parallel atomic units - usually called tasks, scientific applications are classified into:

- Coarse-grained parallel problems

- Medium-grained parallel problems

- Fine-grained parallel problems

Application processes that have large amount of computation between communications generally produce good speedups. They have a high computation to communication ratio and are called coarse-grained but "embarrassingly parallel" applications. If granularity increases beyond the optimum level, the applications will have low computation to communication ratio. If we cannot decouple communication costs associated with increased granularity in those applications, communication overheads may outperform computational advantages of parallelizing the application in a distributed environment. Those applications can further be classified into medium-grained and fine-grained parallel applications, depending upon the fineness of granularity of the tasks.

\subsubsection{Coarse-grained Parallel Problems}

Coarse-grained but "embarrassingly parallel" applications are data intensive in nature. Examples are scientific problems in earth observation [13][14], climate studies [15] and High Energy Physics (HEP) [9][10][11].

\section{Applications in High Energy Physics}

HEP applications are data intensive in nature and they allow large amount of computational work between data transfer and communications. [11] discusses several real world HEP applications using grid tools with data and functional parallelism. It explains the use Large Hadron Collider Computing Grid [16][17] developed in CERN for the scientific community [18]. This grid provides a system for batch processing for HEP applications through a large number of computers connected through Internet. Article [10] investigates the use of an e-Science Grid in solving subtle differences between matter and anti-matter by continuously colliding bunches of high-energy electrons and positrons. The European Data Grid 
is used to manage data intensive HEP applications as described in [19][13][20]. [14] discusses grid middleware services that support data intensive world-wide distributed experiments in HEP using the International Data Grid service.

\section{Earth Observation and Climate Studies problems}

Article [15] describes Earth System Grid prototype that support interactive analysis of replicated data to simulate climate models. The Data Grid project is also looking into Earth Observation Science applications that require large computational power and access large data files distributed over a geographically distributed archive [13][14].

\section{Applicatons in Bioinformatics}

[13] and [14] describe how the Data Grid can process huge data acquired by automated gene sequencers and other experimental techniques to derive biological functions of genes [21]. [22] discusses a grid computing platform for kidney and human physiome modeling that provides experimental scientists with access to computational simulations and human and animal kidney research knowledge databases hosted in separate laboratories around the world.

\subsubsection{Medium-grained Parallel Problems}

The naive N Body problem Solver (NBS) in physics [23][24] is a classical example of a medium-grained but communication-intensive scientific application. NBS is central to the problems involving celestial bodies. It has got implications in nuclear and molecular physics as well. EasyGrid developed by Universidade Federal Fluminense (UFF), Brazil [23] has proposed an application to tackle NBS in Physics. [24] also proposes a solution of a Three-Body Problem in Quantum Mechanics on parallel computers. The Heat Transfer Equation (HTS) is another example of a medium-grained but communication intensive application.

Scientific kernels like the Conjugate Gradient Solver (CGS) for solving linear systems [25] and Cholesky Factorization (CFS) are also present medium-grained but communication intensive solutions. CGS [25] is used to solve linear systems in structural engineering and can be considered to be a medium-grained parallel application. Articles [26] and [27] shed light into the use of CGS in structural analysis. An implementation of solving parallel finite element structural analysis for a dam structure using CGS on a distributed parallel environment is discussed 
in article [28]. Many problems in life sciences can also be solved using mediumgrained but computation-intensive applications.

\subsubsection{Fine-grained Parallel Problems}

The parallel LINPACK benchmark and problems in Computational Fluid Dynamics [29] are examples of fine-grained applications. Very few attempts are made to make these applications HPC enabled using SOA parallel programming models because of the high interdependency of their parallel tasks and the resulting high communication overheads that may grind down their performance.

\subsection{Different Approaches to HPSC}

In finding solutions to above scientific problems, whether it is a simulation in the formulation of new science [30], or computation of nontrivial mathematical, chemical or biological equations, [31][32][15][33][11], scientists wanted uniquely different approaches to satisfy their high performance computing needs. As a result, there emerged different approaches to HPSC.

Different flavours of grid computing dominated the research and academic circles whereas enterprise and business world led the innovations in service oriented computing platforms. There were other contributing approaches like common component architecture and data-flow programming models which finally led to a convergence of technologies. Even though we cannot confine these approaches into water-tight compartments, this section tries to present separate and distinctive accounts of SOA, grid and the other contributing approaches towards HPSC.

Standard approaches to HPSC also include message passing tools like MPI (Message Passing Interface) and its different flavours and shared and distributed memory paradigms like OpenMP. A detailed discussion of these are given in the Related Works - chapter 4.

\subsubsection{Service Oriented Architecture}

SOA is a programming paradigm based on the idea of composing applications by invoking network-available services to accomplish some tasks, mainly adopted by enterprise circles [5]. This distributed computing approach suggests breaking up of otherwise serial computations into smaller atomic units that can be parallelized over commodity hardware computational nodes. Those atomic units 


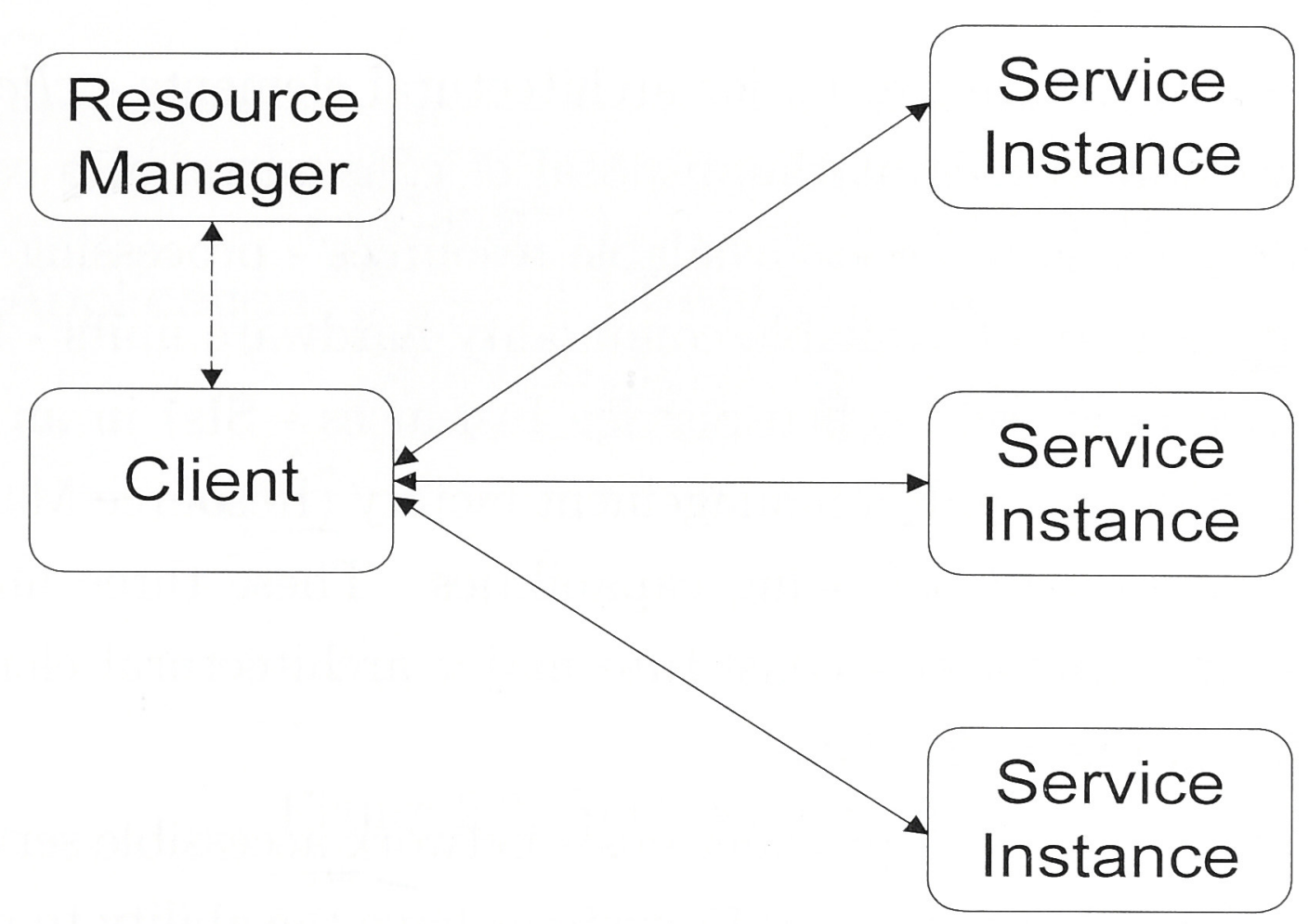

Figure 2.1: The SOA Model

of computation are called services and are considered as the building blocks for applications [34].

[5] defines services as "autonomous, platform-independent entities that can be described, published, discovered, and loosely coupled in novel ways" and "can be assembled to create much bigger and complex applications" [35]. The challenges to run those applications which consist of a collection of sub-applications in a consistent and predictable manner in a distributed environment [36] are met with the help of proper load balancing and scheduling techniques.

SOA can empower an organization with a flexible infrastructure and processing environment by provisioning these services and providing a robust foundation for leveraging these services [5]. It is intended to provide an organic software environment for organizations which support rapid, low-cost and easy composition of distributed applications [5] [8]. The emergence of SOA has been supported by message passing systems such as web services and has its roots in component oriented architecture as discussed in section 2.2.3. Web services popularized SOA and enabled compute resources of an enterprise to interact with each other to perform complex activities using simple http connections over Internet or intranet [34]. 


\section{Architecture}

In the SOA model, there are two major architectural elements - clients and services [37]. Services are placed at the disposal of consumers, also called clients. The client should be able to access available resources - processing power, storage capacity and memory of available commodity hardware units - by initiating instances of service processes (called Service Instances - SIs) in an orderly and effective fashion. This demands a management facility (Resource Manager - RM) with a scheduling and load balancing capabilities. These three units - Client, Service and Resource Manager - constitute major architectural elements of the SOA model (refer to Figure 2.1)[38].

In a typical SOA scenario, a provider hosts network accessible service modules and defines the service description. SOA systems have the ability to publish these service descriptions effectively. The client or the consumer discovers these services with the help of a registry service. This RM module is responsible for resource allocation and load balancing for each application and its runtime needs. Using this mechanism the client and the service nodes must be able to orchestrate conversations to conduct long-lived flexible transactions [38].

\section{Main Concepts and Interactions within SOA}

A consumer in SOA is a client process that is requesting services from compute nodes for an application. The client and service are pieces of software that run on hardware nodes as process instances. A client process can access a number of service instances (SIs). A request of a client to SIs for a particular service along with its input data is called a task. A group of tasks that share common characteristics, such as being part of a single application, constitute a session. An SI can accept infinite number of tasks within a session (refer to Figure 2.2 for a pictorial depiction of these concepts).

Generation of Tasks: Many applications, especially the scientific ones, progress in steps. These steps iteratively process the data and perform the computations to produce final results. Each iteration in this process can be called a generation within the life of the application. There will be a set of tasks that share similar characteristics, doing similar jobs within each generation of those applications. That group of tasks within an generation is called a generation of tasks. 


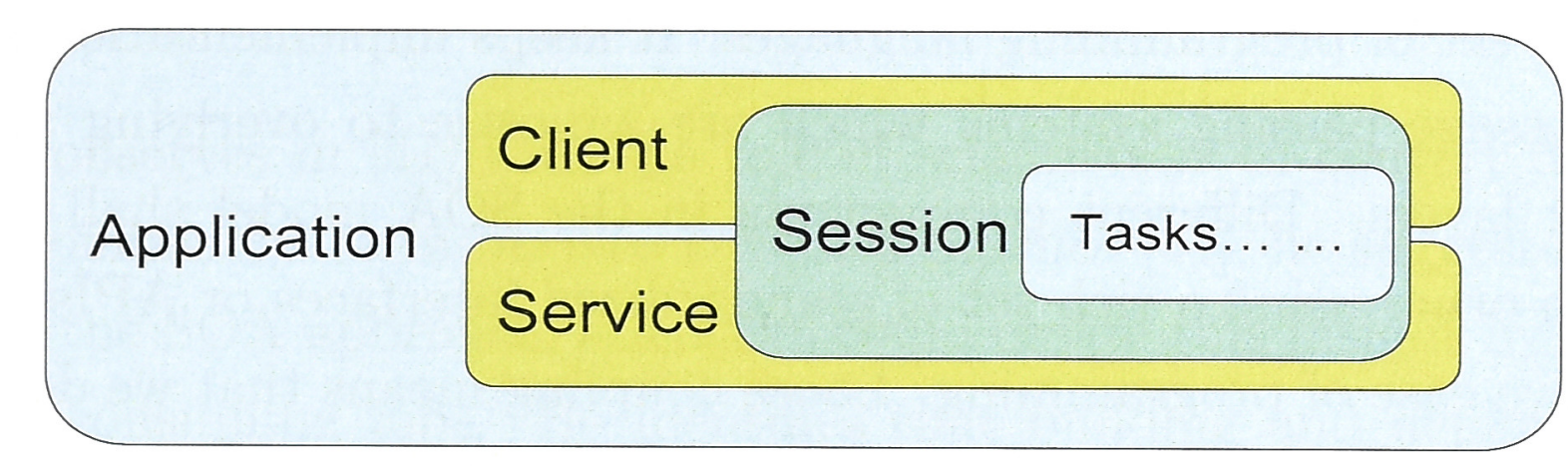

Figure 2.2: SOA Main Concepts

Task Granularity: The time taken to complete a task at the service process is called task granularity. The task granularity is heavily depended on the work load associated with the task. For the same application, using the same algorithm, the task granularity may vary for different executions depending upon the data size given for processing. For example, the task granularity of a Matrix Multiplier application using a matrix of order 2000 will be lesser than that of a matrix of order 4000 .

Interactions: The Resource Manager is supposed to have the knowledge of available resources at any one time. With this knowledge it creates and manages a session where it distributes tasks or batches of tasks to available SIs by following a scheduling and load balancing policy. Once the resources are set by Resource Manager for an application and that information is passed on to the client process, the client process can directly communicate to the relevant SIs. The SIs implement the application logic designed by the programmer by accepting tasks sent from one or more clients, performing computations and then sending back the results to the client process. The task results are collected at the client process to produce final outcome. These actions constitute the compute services of conventional SOA middleware[39, 40].

\section{Characteristics of SOA}

Service oriented architecture is built on concepts such as inter-operability, loose coupling, programmability, flexible configurability, fault tolerance, scalability and quality control [8][5]. 
Interoperability, Programmability and Loose Coupling In order to keep the freedom of different implementers, SOA application development is usually open to large set of programming languages. It keeps implementation neutrality by using message passing systems which are agnostic to overlying application and network layers. Different components in the SOA model shall be able to interact each other using a rich set of standardized interfaces or APIs [41] which ensure relative ease of programming. Loose coupling means that we don't expect a rigid consistency across the various components of SOA. However, a high-level contractual relationship that makes a system-level consistency has to be ensured [38].

Performance Under Heterogeneity and Fault Tolerance The SOA approach is said to be naturally heterogeneity-oblivious. SOA relies on generating more tasks than SIs and service nodes to ensure efficiency under heterogeneous conditions. The Resource Manager can then batch the requests for independent tasks from client process(es), according to the availability and capacity of service nodes and SIs on them. It thus provides a very simple solution to the otherwise difficult problem of dealing with heterogeneity using algorithmic techniques [42].

The key to the fault-tolerance capabilities of SOA lies in the ability of its service processes to persist long enough to detect faults when some of them are terminated for some reason. Once the fault is detected, they could kick start a corrective procedure to heal, abandon, restart or duplicate the faulty process from the history log data [38]. Any failure at the lower level of the hierarchy can be detected by the higher level of management entity. If the higher level process terminates for some reason, that will be detected by the lower level of processes and, after a consensus, one of them could assume the role of the higher entity.

Scalability and Quality of Service The SOA approach presupposes large numbers of networked commodity hardware working together to deliver efficient performance and a large consumer base that uses them. This implies that SOA systems should be able to scale well over a large number of nodes and for a large number of consumers. Service Level Agreements (SLA) are enforced in contracts to ensure Quality of Service (QoS) in almost every service area of businesses. In order to be compatible with business practices and to ensure consistency in quality, SOA should be able to support a SLA based quality control system. This involves quantifying the services offered by service providers and proper pricing of services that are consumed by the consumers[43]. 


\section{SOA in HPSC}

Ian Foster observes in [41] that the role of scientists is to convert data into insight. By coining the term Service Oriented Science [41], he suggested that with the help of the SOA approach, scientists could create this insight by automating many time-consuming jobs. He identifies that building and deploying services for a scientific domain require three things - the domain-specific content, the domain-independent software functions and physical resources, of which the last two - functions and resources - can be handed off to specific providers, leaving the first area - domain-specific scientific content - to the scientists, which is their expertise. Domain-independent software functions can be provided by SOA for scientific computing. SOA can also simplify the programming efforts of the scientific community to generate domain-specific content by making available powerful tools to create scientific processes as services over a network.

But not all scientific computing needs SOA. Sriram Krishnan et.al. [44] observes that "understanding how to leverage these developments to actually design and build a $(S O A)$ system remains more of an art than a science." If the requirements are relatively well understood and stable and if the set of resources are fixed or a single interface to the applications is sufficient for the user community, they observe that it is sufficient to go for a traditional approach rather than SOA for HPSC.

Nevertheless, the interest to use SOA to run scientific applications is growing $[34,45]$. It is generally understood that SOA has the potential to empower scientists and researchers to seek solutions to many unique problems they face in their scientific domains. SOA empowers them to design custom built applications for their specific needs, access distributed resources and orchestrate the whole process in a fault-tolerant and efficient manner [46]. [34] suggests mapping of MPI interconnects to web services to enable SOA for scientific computing. Even though the SOA implementation for scientific applications needs more maturity, the Linked Environments for Atmospheric Discovery (LEAD) project, as explained in [36] and [38], gives us an insight into how a scientific use-case can be addressed using a service oriented approach. Some of the enterprise SOA middleware like Platform Symphony try to use the SOA approach to solve scientific problems that need high performance outputs [47][10]. 


\subsubsection{Grid Approach}

The history of grid computing is closely linked with the abundance of cheap commodity computing hardware in the research labs and university houses. Software architectures have emerged to exploit those new possibilities by sand-boxing the complexity of computation as small parallel units called meta-computing [48]. Later on it was evolved into grid computing [49][50]. It is a distributed computing model which orchestrates distributed computing resources of different academic and research institutions to work like a single, unified computing system [51]. This has empowered scientists and researchers not only to harness relatively cheap computing facilities from around the globe [11] but also to share scientific instruments and resources like sensors in thermodynamic studies [52], DNA data repositories in bio-informatics [53] or even the Large Hadron Collider at CERN [9], without geographic boundaries.

\section{Grid Middleware}

"The Globus Alliance" initiated by Argonne National Laboratory remained as the pioneering and most successful venture in providing grid infrastructure for research community grids [54]. An outline of a service oriented grid system has been dealt with in detail by Ian Foster and Carl Kesselman as early as in 1997 [48]. They developed Globus grid toolkit - an open source but not vertically integrated software toolkit. Its idea is to provide a "bag of services" which can be used by developers to create their own grid systems.

At the same time, there were a few other serious attempts to bring about scalable and stable grid system tools. Examples are Legion, which was developed by the University of Virginia [55] and Unicore - an effort of the German Ministry for Education and Research in co-operation with ZAM, Deutscher [56]. Legion is an object-based grid middleware system. It is structured as a system of distributed objects operated on by active processes that communicate using a uniform remote method invocation service. Unicore has a three-tier architecture consisting of Client, Gateway, and multiple instances of Network Job Supervisors (NJS) and Target System Interface (TSI).

One of the recent entry in to grid system middleware system was Gridbus (recently rebranded as Cloudbus) by Melbourne University [54] which aims at the commoditisation of grid services with the idea of a computational economy. Gridbus is not a vertically constructed grid middleware system, that is it does not provide all functionalities of a grid system by default. Many of its components 
are built on existing grid tools. For example, the Gridbus brokerage system is built on Globus tools and has employed a Unicore adapter [54]. One of its offshoots - Aneka middleware - provides a service oriented platform for desktop grid computing (for enterprises, where PCs are brought together to give high performance results) using the .NET platform.

There were also efforts to use message passing systems such as MPI as an alternative to provide efficient grid-enabled applications. A serious attempt in this regard is that of EasyGrid developed at Universidade Federal Fluminense (UFF), Nitero'i, Brazil [23].

It is interesting to note that many of these have made successful transitions to become cloud computing tools in very recent times as discussed in section 2.3.

\section{Classifications}

Grids can be classified on the basis of their nature and purpose. In line with the classification of scientific applications into data intensive or computation intensive applications (as mentioned in section 2.1), grids can also be classified into computational grids or data grids. Computational grids are computation intensive grid systems and examples are NASA IPGrid for aerodynamic studies and NSF TeraGrid for e-Science [15]. Data grids are data intensive grid systems which need to transfer or replicate huge amounts of data from one node to another in a distributed fashion. An example would be European Grid at CERN which deals with the large amounts of data coming out of Large Hadron Lab [51][13][9]. However, data grids always incorporate the core functions of computational grids. In other words, all data grids are also computational grids but not all computational grids are data grids.

Even if the purpose and nature of these grid systems varies, there are some core elements which are common to all grid systems. Those core components of a grid system form the grid-middleware to provide a grid fabric for specific grid applications.

\section{Architecture}

Contemporary grid infrastructure is inspired either by SOA or Common Component Architecture (CCA). Accordingly, there is a necessity to insulate the parallelized atomic unit of work - tasks - to perform independently on resource nodes. These tasks have to be managed in different levels with the help of Resource Manager, to perfectly orchestrate the work-flow. Suitable scheduling techniques 
and runtime system performance evaluations are to be employed at the resource manager level to ensure efficiency in grid environments. All these interactions have to be done in a secure and fault-tolerant fashion. In an enterprise grid, the services should be able to conform to a minimum SLA and there should be means to quantify those services. These core components in relation with existing grid infrastructure are discussed below.

Resource Management Resource management means efficient management of available resources (processor time, memory, network, storage, etc.) of available resources in the grid environment to give the best possible results.

Globus Resource Allocation Manager (GRAM) within the resource management package of the Globus grid toolkit provides remote execution capability and reports status for the course of the execution. In Unicore, resource management is done with the help of Network Job Supervisors (NJS) that process Abstract Job Objects (AJO) with the help of Target System Interfaces (TSI). AJOs are Java classes that encapsulate jobs in an abstract form within Unicore semantics. Melbourne University's Gridbus project uses a Gridbus Broker to manage grid resources and is built on basic functionalities provided by Globus. They have also introduced a Unicore adapter for Gridbus Broker. Gridbus also provides Libra a cluster economy and resource allocation system that guarantees a certain share of the system resources to a user job. This will enable the job to be completed by a user specified deadline if it has got enough monetary budget for it [54]. A stand alone grid resource management system, Nimrod-G, has been developed by University of Monash, Australia. This grid broker provides economics-driven access to low-level grid services using its four key components - a task-farming engine, a scheduler, a dispatcher, and agents [57], [51]. Based on SOA principles, it provides mechanisms to implement QoS parameters based on an SLA and allows its run time trade-off to delay or accelerate computational rates for varying prices.

Information Services Information services take an important role in grid computing because grid operates in a highly heterogeneous environment and the resource nodes are not completely dedicated nodes for grid use alone. The network parameters may change at runtime according to network traffic. The availability of a node and efficiency of running tasks on a nodes may vary according to the load on the computer node caused by other programs or applications. This scenario demands better monitoring of resources - network, memory, computational 
speed and storage - to provide an efficient grid implementation.

The Globus component of information services is called Monitoring and Discovery Service (MDS) [58]. MDS has a three-tier structure - Information Providers (IPs) at the bottom, Grid Resource Information Service (GRIS) which is a singleton and a Grid Information Index Service (GIIS) that records resource information provided by other GRISs and GIISs.

Legion is structured as a system of distributed "objects". All hardware and software resources in a Legion are represented as Legion objects. Legion uses Context Objects to map context names and associated status information about resource nodes for the benefit of runtime scheduling and load balancing [54][58].

Security, Authentication and Fault Tolerance Resilient security measures are needed for reliable functioning of any grid systems. Globus provides Grid Security Infrastructure (GSI) for authentication of grid users and secure communication. It is built with a combination of SSL (Secured Socket Layer), PKI (Public Key Infrastructure) and X.509 Certificate Architecture (CA). In GSI, Grid CAs issue certificates that are to be trusted and verified by the remote nodes to allow communication[54] [55].[59] introduces SHARP - a framework for secure resource management for networked computing platforms. SHARP provides security in the form of tickets and leases. Since it has to work with system without global trust, it tries to mitigate the risk by allowing access to slices of resources to limited period of times [59]. Grid infrastructures generally provide a user ID and password based security system for authentication of its users. They are also supposed to provide a reliable and fault tolerant infrastructure with no single point of failure.

QoS and SLA Maintenance With the evolution of enterprise grid and the emergence of the idea of computational economy, QoS and SLA started to take centre stage in grid computing[54]. This is achieved with the help of runtime information about resources' statuses and dynamic scheduling changes based on the information provided by information services. [43] suggests a workflow process managed QoS maintenance system for enterprise grids. 


\subsubsection{Other Approaches}

\section{Common Component Architecture}

The Common Component Architecture (CCA) forum proposed a component based model over an object oriented model [60]. The basic principle is to encapsulate units of functionality in a black-box that can be composed together to form applications as components. The CCA then provides interfaces for communication between those components. Those abstract interfaces are called ports in CCA. Important ports are standardised by the CCA Forum [61][35].

Since a component is independent in itself and can communicate with other components through component interfaces, this model assures component-level and programming language level interoperability [62]. Frameworks are envisaged to manage CCA components to create applications. They provide a set of standard services that are available to all components with the help of an Applicaton Programming Interface (API). The API supports interaction between components and various builders for functions such as notifying components that they have been added to a scenario or deleted from it, redirecting interactions between components, or notifying a component failure. Earlier adaptations of the CCA are found in software frameworks such as JEE and CORBA [63][35]. The CCA model is successfully used in computational hydrodynamics [62], quantum chemistry, combustion modelling and climate modelling [61].

\section{Dataflow Programming Model}

The Dataflow programming model abstracts the process of computation as a dataflow graph consisting of vertices and directed edges. This model has been implemented in Melbourne Universitiy's Aneka [7]. This model is implemented through three key services: 1) a Registry Service that maintains location information of vertex data 2) a Dataflow Graph service that maintains the data dependency graph for each job and 3) a Scheduling service that distributes tasks to available resource nodes or workers[7]. The vertex data helps the scheduler in dataflow model to distribute work to the workers. The vertex consists two entities: the data created during the computation or the initial input data and the execution module to generate the corresponding vertex data. The directed edges indicate dependencies between the vertices. The scheduler will be responsible for monitoring the status of each worker, dispatching ready tasks to suitable workers and tracking the progress of each job according to the data dependency graph. 


\subsection{Convergence in Cloud Computing}

The complementary nature of SOA and grid computing has been identified by Global Grid Forum (GGF), the oldest and largest organization that represents both the scientific and industrial community in defining the standards for Grid technology [36]. It has proposed standards for a service oriented grid through the Open Grid Service Architecture (OGSA). OGSA standards have been used by GGF to develop the most commonly used open-source software toolkit for building grids - the Globus Toolkit [49]. This convergence have been taken further forward by the arrival of the cloud computing paradigm.

The emergence of large and medium sized data centers powered with cheap but powerful intra-network connectivity and also connected to the outside world with a relatively fast Internet provides the infrastructure rationale for the cloud computing paradigm. Initially these data-center were built for various enterprise and business applications and services. The best example is that of Amazon data centers which were built to support the online publishing business. But the unused compute cycles and hardware capacity of those data centers warranted better use. Moreover, those data centers can be built on commodity storage and networking hardware. It makes it easy to sell these resources on a cheaper pay-asyou-go model relying on statistical multiplexing among large number of customers relying on economies of scale [64] [65]. On the technology front, even though virtualization technology was around for a long time, it was getting mature enough to support multiple hardware-agnostic operating system (OS) installations on the same physical machine. Internet connectivity using optical cables and satellite transmissions created a perfect setup to envisage logistically distant compute resources to be virtually available to customers whenever and wherever it is needed. Service oriented enterprise computing has already succeeded in transforming business workflows into manageable service modules with the help of Web 2.0 and web services technology [66].

The cloud computing paradigm emerges as a synthesis to hardware and software dialectics in computing history (refer to Figure 1.1) for it adopts a model which conceives everything as services. At the same time those services transcend the boundaries of any single enterprise or entity built around physically together resources - data centres - with a mix of commodity and specialized server hardware, networked together using distributed and parallel computing principles. Even though the enterprise community is more enthusiastic about it, both enterprise and research circles seem to embrace it [66][67][68][69]. It has also almost 
certain prospects of changing computing as a utility after water, electricity, gas, and telephony [70].

\subsubsection{Definitions and Classifications}

\section{Definitions}

Within the research community, important advancements has been made by Ian Foster et. al at Argonne National Laboratory [65], Rajakumar Buyya et. al at Melbourne University [70] and Armburst et. al at Berkley University [66] [67], who present similar but distinctive definitions to cloud computing. The National Institute of Standards and Technology (NIST) in the USA has made a successful attempt to define cloud computing for the business community towards legal and compliance purposes[71]. These definitions give us substantial insight and a level of clarity into the evolving nature of cloud computing.

From the definitions in above works, it is fair to conclude that cloud computing is a form of parallel and distributed system which uses virtualization techniques to orchestrate large storage, memory and network resources of data centers or similar resources as a unified unit but with apparent elasticity and available ondemand to customers. It relies on the economies of scale to provide infrastructure, platform or application services without upfront cost to customers over a network or Internet with minimal management, running and maintenance costs.

\section{Classification Based on Service Model}

The general consensus is to classify cloud computing paradigms based on their service model as Infrastructure as a Service (IaaS), Platform as a Service (Paas) and Software as a Service(SaaS) [71][70][65]. The present cloud computing stack according to this classification is given in Figure 2.3.

IaaS This is the layer with lowest level of abstraction. It gives consumers the ability to provision storage, network and other basic computing infrastructure with limited control over its hardware and networking components (e.g.: using some parameters that can be adjusted using a web interface). However the consumers are not responsible for managing and maintaining those resources, which is taken care of by the cloud provider. The broad spectrum of services that come under this category consists of HaaS (Hardware as a Service), DaaS (Data Storage as a Service) or CaaS (Communication as a Service) [64][70]. 


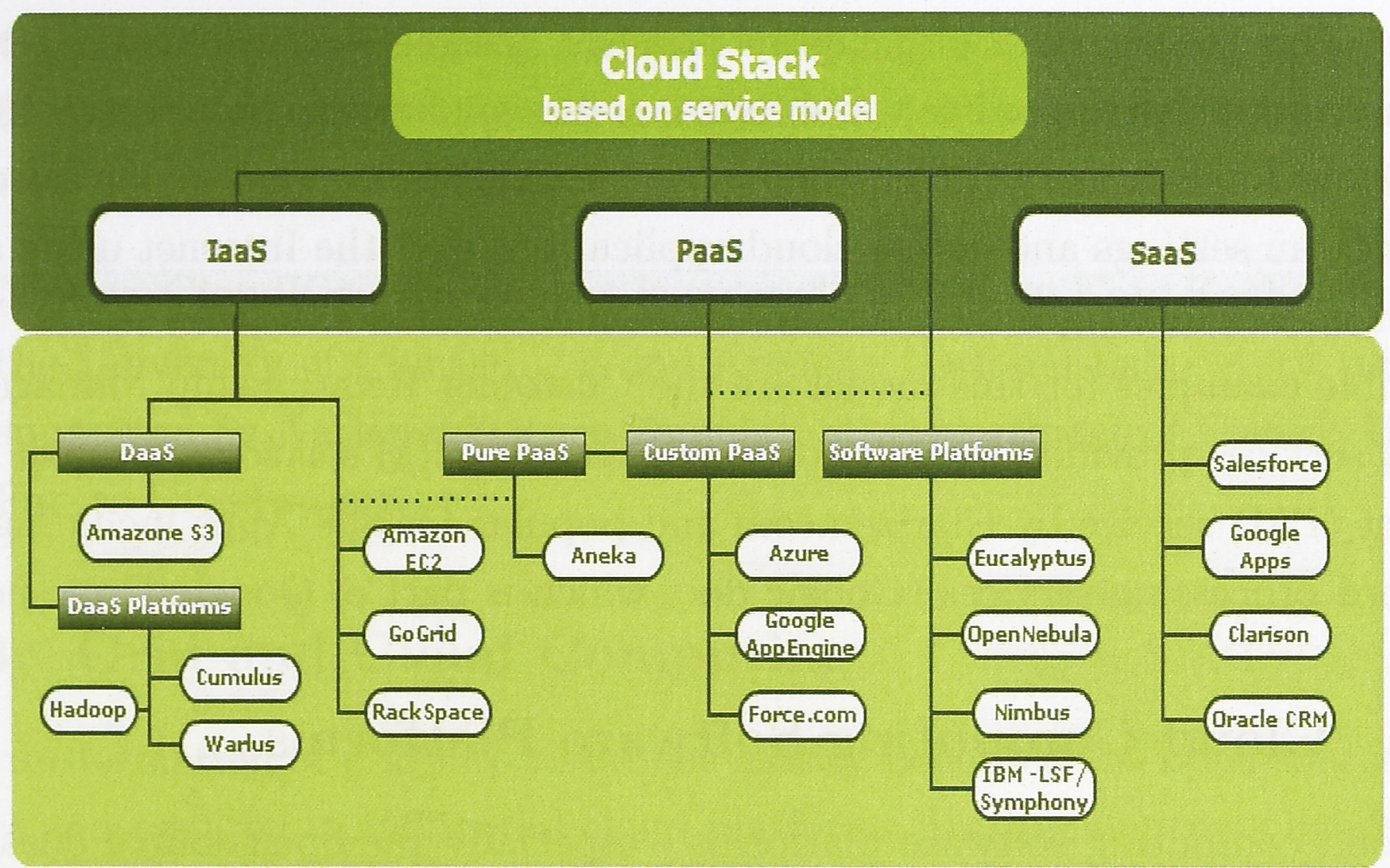

Figure 2.3: Cloud Computing Stack: Based on Service Model

The major providers in this area are Amazon S3 and EC2, GoGrid, RackSpace, etc. Amazon S3 (Simple Storage Service) provides just storage services whereas EC2 (Elastic Computing 2) gives live but virtualised machines which can be spawned from the default disk images of popular OS kernels and its flavours. GoGrid provide services similar to EC2 with some additional features like provision to buy load balancers for GoGrid cloud clusters. The data file system platform - Hadoop on Cloud or Yahoos Pig-services can also fall into this category.

PaaS This layer provides a higher level of abstraction where the cloud provider provides not only the infrastructure but also the basic platform to run cloud applications on the top of that infrastructure. There can also be pure PaaS providers who reply on other IaaS providers to set-up their platform. The platform could involve operating systems, custom libraries, support for computing languages, middleware and other tools provided by the cloud provider. The customers may have some control over the application-hosting environment but not with its underlying infrastructure.

The major PaaS providers at the moment are Microsoft with its . Net based Azure platform, Google AppEngine, Force.com with its Cloud Software Environment Layer services and relatively small and unique domain platforms like ManjaraSofts Aneka. 
SaaS This is the layer with highest level of abstraction on cloud. Here the customers are only exposed to the software or the application that sits on the top of IaaS and PaaS layers. The customers are agnostic of the underlying hardware and platform settings and access cloud applications over the Internet using a web browser or a light-weight interface application on their desktops.

Visible examples for this model are the Customer Relationship Management (CRM) services provided by Salesforce and Oracle CRM services, Project Management (PM) service by Clarizen.com and popular Google Apps including mail and word processing services (Google doc, which is part of Google Drive now).

\subsubsection{Cloud Computing Software Platforms}

The cloud computing software platforms - especially the open-source ones - deserve special mention because they enable viable private, community and hybrid models for small to medium enterprises and research/academic organizations.

The big players in cloud computing like Amazon, Microsoft, Google, IBM etc implement their own enterprise cloud software and publish a set of tools like SDK, APIs, GUI, web interface, etc for their customers to access and work on their cloud infrastructure. But the actual code remains proprietary, which restricts others to implement, customise or modify their own cloud infrastructure. The only option remains is to buy or lease those proprietary softwares and to accept technical support from its owners to set-up, customise and run a cloud infrastructure. Microsoft Private Cloud, IBM Cloud Service Provider Platform, Platform Computing LSF and Symphony, ManjaraSofts Aneka [72, 73] are examples of propriety Cloud computing software platforms that can be used to implement a private or hybrid cloud infrastructure. Almost all of the above platforms have made their transformation from SOA or enterprise grid middleware products to cloud computing products.

On the other hand, we have couple of open-source cloud software platforms that have enough maturity, sophistication and convenience to provide reasonably good cloud infrastructure for interested parties. Most of them have developed in research circles as a natural transformation from grid computing platforms to cloud computing platforms. Important and noteworthy of them are [68]:

- Eucalyptus: It was developed at the Computer Science Department at the University of California, Santa Barbara as part of Virtual Grid Application Development Software Project (VGrADS) funded by the National Science Foundation. 
- OpenNebula: OpenNebula was first established as a research project back in 2005 by Ignacio M. Llorente and Rubn S. Montero at the Distributed Systems Architecture Research Group at Universidad Complutense de Madrid.

- Nimbus CloudKit: The project is created and led by Kate Keahey et al. at the University of Chicago [74] and Argonne National Laboratory, and it is supported by National Science Foundation, Department of Energy, US and Future Grid project.

\subsubsection{Grid and Cloud Computing}

The transformation of grid into cloud has mostly benefited the open-source community and resulted in influential cloud platforms like Eucalyptus, OpenNebula and Nimbus. Very few grid initiatives have made a move from open-source to proprietary cloud products. The most important of these is Aneka Cloud Platform by ManjaraSoft - a start-up from Melbourne University, which made its move from Gridbus project to Cloudbus project. In another development, C12G Labs - an offshoot from OpenNebula project - is distributing OpenNebula Pro a commercially supported variant of OpenNebula. OpenNebula itself has evolved from Globus grid project. Eucalyptus is successful in providing an Amazon compatible API, by with it has taken a special place as an-open source on-premise implementation of Amazon EC2 cloud infrastructure[68][75].

\subsubsection{SOA and Cloud Computing}

The enterprise community has contributed to cloud computing through its SOAdriven research and products while the research and academic community has contributed though its grid computing efforts to cloud computing. The very concept of computing modules as services has deep roots in the philosophy of SOA. But in the cloud, the scope and extent of service is broadened to a new level [76]. Mladen A. Vouk et.al acknowledges that the "powerful underlying and enabling concept (of cloud computing) is computing through service oriented architectures (SOA) [77]. Web Services and Web 2.0 were the two technologies that drove the SOA and Ian Foster et al points out that the "cloud more tends to do what Web 2.0 was able to do. The cloud computing sits on Service Oriented Architecture, according to Ian Foster as he described it in Figure 1 of the article [65]. In demonstrating the relationship of cloud to SOA, Lamia Youseff et al notice that, "cloud applications can be composed as a service from other cloud services 
offered by other cloud systems, using the concepts of SOA" [64]. In explaining the Aneka Cloud platform, Rajkumar Buyya et.al suggest, "the (cloud) framework is based on an extensible and service oriented architecture that simplifies the deployment of clouds [73].

\subsubsection{Cloud Computing for HPSC}

The growth of cloud computing in the recent years have prompted the research community to experiment the feasibility of HPSC on cloud platforms and also to propose new middlewares to deal with cloud HPSC scenarios.

Tomasz Wiktor Wlodarczyk et. al. in [78] presents a survey of motivations and recent attempts in research circles to use cloud computing for high performance results. It suggests that "motivations are mostly two fold. First, focusing on lowering the cost of resources and second, improving the ease of access to resources". The research paper [79] discusses the deployment, performance and cost efficiency issues of HPC applications on the three major cloud IaaS - Amazon EC2, Azure and Rackspace - using NAS parallel benchmarks and compared them against the performance on a cluster. They found that "even though all clouds present performance issues" when more than 4 nodes are used "Azure has a better performance than the other clouds and the cluster, with EC2 and Rackspace having a slightly worse performance than the cluster". They conclude that "Our analysis showed that the cloud can provide a viable platform for running HPC applications, despite some disadvantages in the deployment." Alexandru Iosup et. al. published performance analysis of Many-Task Computing based scientific workloads on cloud infrastructures in [80]. They used well-known microbenchmarks on four Selected Cloud IaaS: Amazon EC2, GoGrid, ElasticHosts, and Mosso and results showed that "an order of magnitude better performance is needed for clouds to be useful for daily scientific computing." However they found that "clouds are reasonably cheap for scientific computing, if the usage and funding scenarios allow it" and they are "a viable alternative for short deadlines".

The SciCloud project at the University of Tartu, Estonia proposes a distributed computing framework, Stratus, which is designed to support complex iterative scientific computing algorithms in the cloud [81]. The novelty of Stratus is that it uses Bulk Synchronous Parallel (BSP) model of strict barrier synchronisation than the conventional MPI or MapReduce models to deal with those algorithms. Stratus architecture consists of a Cluster Manager (CM) and number of Task Managers (TM). The CM start, divide and maintain tasks and allocate 
resources in the form of TM instances in the cloud IaaS. TMs execute tasks/subtasks and manage communication between themselves at synchronisation points. The research paper provides results of implementing Conjugate Gradient algorithm using BSP model on Stratus and compares it against MPJ Express and mpiJava MPI communication libraries for JAVA. The framework is still in a prototype state and they plan to build their own BSP core library in JAVA for the future.

e-Cloud project at the Universidad de los Andes, Colombia aims to provide scientific SaaS solutions on cloud IaaS [82]. It is designed as a scientific PaaS on cloud which manages the on-demand provisioning and configuration of computing instances, storage, application management, job scheduling and monitoring, and data management. The users access their private workspace using e-Cloud Web Portal using a username/password credentials. Users can create Virtual Clusters (VC) which are managed by e-Cloud Resource Manager(RM). The RM executed the actions on VC on a public IaaS, using e-Cloud API. They have implemented first version of e-Cloud on Amazon EC2 and demonstrated that BLAST algorithm can run on it economically, even though it may suffer from performance issues.

University of Electronic Science and Technology of China, Chengdu, China ventures to design CloudDragon, a scientific computing Cloud platform based on OpenNebula [83]. They use Swift scientific workflow management system (SWFMS) as the Cloud gateway service to help coordinate and automate scientific analysis and discovery using a Cloud Resource Manager(CRM). The CRM accepts resource requests from SWFMS, and interfaces with OpenNebula (via APIs) and dynamically provision virtual clusters to the workflow service. They demonstrate the functionality of this architecture without specific reference to it performance results.

\subsection{Case Study: Platform Symphony}

The PhD thesis of Songnian Zhou at UC Berkley in 1987 - "Performance Studies of Dynamic Load Balancing in Distributed Systems" [84] - became a catalyst for him to establish Platform Computing in 1993, a provider for enterprise level HPC grid products. Platform started with LSF - a static batch workload manager of HPC clusters in 1992 [85]. In 2001 Platform added Symphony as a dynamic workload management tool which was then transformed into Service Oriented Application Middleware (SOAM) capable of grid-enabling of mission-critical fi- 


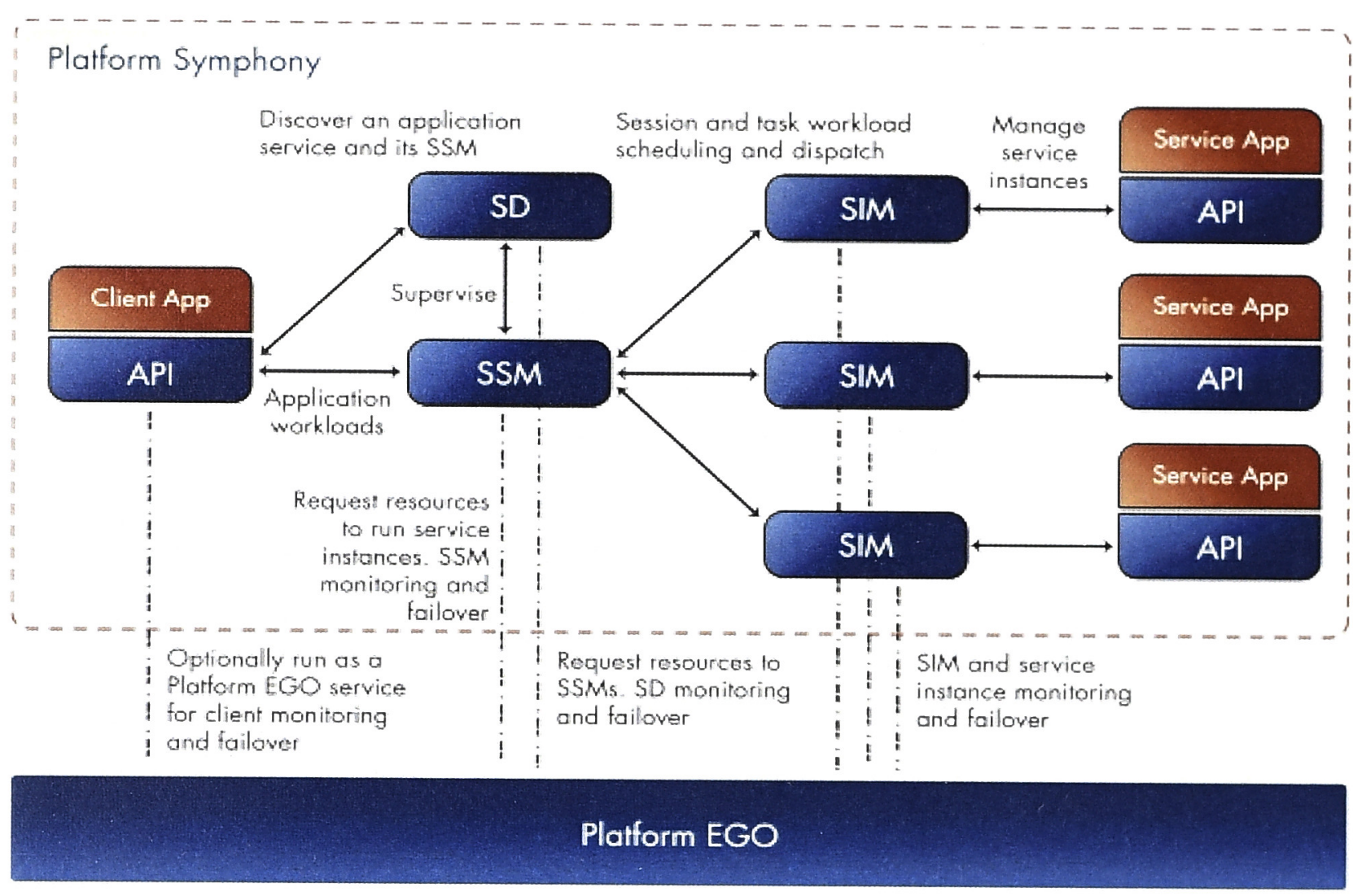

Figure 2.4: Platform Symphony Architecture

nancial applications. Based on the service oriented architecture, it enables an enterprise to run business applications (for example, Monte Carlo simulations for banks) on their compute resources.

\subsubsection{Architecture}

Platform Symphony implements a service oriented architecture where the application logic is encapsulated into one or more software programs called services and can be accessed by client specific application logic. Client instances access the Service Instances (SIs) through tasks with input and/or output messages within a session.

Platform Symphony would accelerate computations within an application by distributing them in parallel to nodes on a grid where these computations would have previously executed sequentially. Techniques for parallelizing applications involve finding computations that take place inside loops and altering application logic slightly so that they can be parallelized. Another common technique is to provide data segmentation and allowing different tasks to process subsets of input data concurrently [86].

The architecture of Platform Symphony consists of client and service application modules and a Resource Management and Scheduling regime consist of Service Session Manager (SSM), Session Director (SD) and Service Instance Manager (SIM). A graphic representation of these core SOA concepts are given in Figure 
2.4 as given in the Platform Computing document [6]. The SSM is responsible for scheduling the service oriented workloads that consist of sessions, services, and tasks. There is one SSM per application responsible for scheduling sessions and tasks from the client(s). The SD is responsible for supervising SSMs and directing clients to their designated SSM. The SIMs which run on each compute host will start SIs and handles interactions between SIs and the SM [86][39].

\subsubsection{Strengths and Challenges}

\section{Strengths}

Platform Symphony is one of the few enterprise middlewares that has been proven convenient enough to application programmers in the financial sector [86]. It also provides a user-friendly application development SDK and API necessary to develop these applications [40]. Moreover, its impact quotient-has been increased by its recent (January 2012) acquisition by IBM to make it as part of its HPC Cloud Suite.

It provides a Common Data service, which avoids sending information needed by each task repeatedly to a service instance. Symphony 5.1 supports sending only a single copy of Common Data to each multi-core compute node, even if multiple SIs run on the same node. It improves the memory and bandwidth efficiency.

Platform Symphony assures fault tolerance with multiple layers system management regime consisting of Symphony Session Managers (SSM), Session Directors (SD) and Service Instance Managers (SIM). Any failure at the lower level of the hierarchy will be detected by the higher level of management entity and will be either healed or restarted. If the higher level manager is terminated for some reason, that will be detected by the lower managers and after a consensus among them, one of them assumes the role of the higher manager [39]. The innovative scheduling and load balancing techniques employed in the Symphony broker also ensures efficiency under heterogeneous environments [6].

There are opportunities for Platform Symphony in cloud computing, which was evident in IBM making it as a part of its cloud strategy [87]. Like many other SOA middlewares, it could sit at the PaaS level in the cloud computing stack. 


\section{Challenges}

Platform Symphony is successful in providing high performance results with high CPU efficiency, high task throughput for large message sizes with optimum task runtimes [88], which is the typical scenario in financial applications. However, the efficiency drops considerably as the task granularity falls beyond optimum levels, because of the communication overheads [88]. There were attempts to use Platform Symphony for scientific applications like BLAST [47]. Efforts were taken as part of this PhD research to test the adaptability of Platform Symphony for scientific applications using a Matrix Vector Multiplier (MVM) application. Preliminary experiments conducted at the Department Computer Science, ANU labs signalled that the Platform Symphony MVM performance decreased considerably beyond the optimum task-time range of $0.05-0.2$ seconds, which can be attributed to task startup and the communication overheads. This was reported in my previous paper [89].

\subsection{Chapter Conclusions}

The above discussions reveal that the high performance computing needs of enterprise and academic circles are addressed by SOA and grid computing, respectively. The academic community appreciates the convenience and effectiveness of SOA model and was successful in adopting SOA principles into many of its academic grid systems. However, SOA's scope and usefulness remained limited mostly to "embarrassingly parallel" scientific applications or kernels. The communication and other overheads restricted "not so embarrassingly parallel" scientific applications to be efficient on SOA platforms. There is a genuine need and scope to come up with solutions to address these issues of SOA to avail its many benefits to academic and research world. The case study of Platform Symphony shows that a SOA middleware based on its architecture could emerge as a solution to address these challenges, if it can produce HPC results. This solution could also help find $\mathrm{HPC}$ solutions for scientific applications in the emerging cloud computing platforms. This PhD research introduces ANU-SOAM - a middleware implementing the architecture and APIs of Platform Symphony, with a Data Service extension to address these performance challenges. 


\section{Chapter 3}

\section{Related Work}

The prospect of high performance computing for scientific applications has opened up a wide range of architectural and technological innovations. The core challenges were to enable efficient communications within the infrastructure and to make tasks independent of each other to ensure optimum granularity of task loads and to sandbox them as services. Many have tried to address these issues from different angles. These innovations consist of efficient message passing interfaces, shared memory tools and distributed file systems.

\subsection{Message Passing Systems}

\subsubsection{Message Passing Interface}

The Message Passing Interface (MPI) has been identified as the standard programming tool for parallel programming. It was initiated by William Gropp et. al of Argonne Labs and is a language independent communication specification standard for parallel programming [90]. MPI-II supports remote memory access [42] to certain levels and can also serve as a viable message passing backbone for grid and distributed computing. It implements both point-to-point communications and collective communications (for example: scatter-gather). The MPI uses the notion of 'communicators' and 'groups' to decide which collection of processes may communicate with each other. It provides both blocking and non-blocking send/receive operations and supports most of the major data types and allows grouping message types together as derived data types.

Usually, MPI applications are not autonomic applications, which means they are agnostic and non-responsive to the varying capacities of the underlying re- 
sources they use. For a homogeneous cluster this model should be efficient enough. However, for a heterogeneous system like a grid for scientific computing, the efficiency and availability of resource nodes may vary from time to time. Varying network strength and network traffic behaviours will also affect the efficiency of applications. The native MPI paradigm does not take care of these influential parameters in a complex heterogeneous system. MPI also does not provide default fault-tolerant mechanisms. In many cases, MPI programming using its APIs is too complex for ordinary scientists who are not professional programmers. Because of these reasons, [34] observes that "a way to deal with this is to move toward a Service Oriented Architecture (SOA)". ANU-SOAM tries to address these issues by providing a SOA infrastructure with its easy-to-use APIs and efficient load balancing techniques while keeping MPI as its communication backbone to take advantage of its high performance results.

\subsubsection{EasyGrid}

Efforts have been made at Universidade Federal Fluminense (UFF), Nitero'i, Brazil to make MPI system-aware to deal with its innate inefficiency towards heterogeneous systems. It resulted in the development of EasyGrid which is designed as an Application Management System (AMS) for the MPI library [91]. The EasyGrid AMS is built on the core middleware functionalities offered by the Globus Toolkit [92] and is embedded automatically into a user's parallel MPI application without modification of the original code. This approach reduces the coding effort of the application programmer by transferring those extra coding efforts to the developer of the middleware - who is supposed to be more competent to take that effort [93].

The AMS is made system-aware by introducing a Resource Management System (RMS) [23]. The RMS collects runtime information from nodes and then employs a hybrid scheduling policy for resource allocation [94]. The hybrid scheduling policy combines the benefits of static and dynamic scheduling. The static scheduler makes use of some sophisticated heuristics. A performance model of the parallel application is represented by directed acyclic graph (DAG) where nodes denote tasks and edges denote communications. With this initial information, an initial scheduling schema is prepared. At runtime, the dynamic sub-scheduling system makes runtime scheduling decisions on the basis of runtime feedback [91]. The dynamic scheduler fine-tunes the initial static schedule by moving tasks assigned to one node to another or rescheduling communications altogether. This 
hybrid model is designed to improve the performance of each application in a heterogeneous environment. This has been experimented and positive results have been recorded in [95], [96] and [91].

The core RMS elements are discussed in [96] and [23]. Each EasyGrid RMS is a three level hierarchical management system consisting a Global Manager (GM), Site Managers (SM) and Host Managers (HM). The Global Manager (GM) is a single entity at the top level and supervises all resource sites in the grid. The Site Managers (SM) at each grid site are responsible for the allocation of resources to application processes. The Host Managers (HM) are responsible for scheduling, creation and execution of the application processes associated with each compute host. Articles [95] and [93] give us an extensive account of the scheduling policies of AMS.

The creativity of EasyGrid is its ability to provide a self-scheduling policy for native MPI applications without extra coding effort. This is achieved by the introduction of RMS which employs a hybrid scheduling policy based on runtime information from resource nodes. The hybrid scheduling techniques implemented by EasyGrid are done directly on top of a generic MPI applications but without the SOA model and an extended Data Service as envisaged in ANU-SOAM. This makes it easier for EasyGrid to implement load balancing scheduling schemas because it does not need to deal with data consistency and integrity challenges raised by a data service associated with each service instance. ANU-SOAM tries to provide similar load balancing capabilities but also keeps Data Service capabilities alive. In that sense, the load balancing and scheduling efforts of ANU-SOAM is unique and superior to that of EasyGrid scheduling efforts. It is also not clear that EasyGrid can adequately address the problems associated with granularity of tasks and interdependency of tasks found in scientific applications.

\subsubsection{HeteroMPI}

The project HeteroMPI (HMPI) is aimed at providing an extension of MPI for programming high-performance computations on heterogeneous networks of computers [97]. It was initiated by the Department of Computer Science at University College Dublin, National University of Ireland. It allows application programmers to describe a performance model of the implemented applications using performance model definition language. The information includes number of processes, volume of computation to be performed by communication groups in the MPI application, data volume to be transferred, order of execution, etc. This informa- 
tion will be used to automate the process of optimal selection of communication groups in MPI that execute heterogeneous algorithms faster than any other group [42], [98]. Recently, they have optimised generic collective MPI communications (for example, MPI_Bcast). HMPI does it by differentiating the message size threshold of collective calls into small, medium and large and then tweaking their implementation to avoid MPI_Gather time escalations and MPI_Scatter leaps in the execution of collective calls [99].

ANU-SOAM is developed on the top of an MPI infrastructure layer. Therefore any improvement to the underlying MPI infrastructure will only benefit ANUSOAM implementation. The creativity of ANU-SOAM lies in the independence of its service tasks to that of that of service instances, which allows more than one task per SI. While HMPI tries to solve the problem of heterogeneity by grouping the right compute processes as communication groups, ANU-SOAM tries to achieve it by sending weighted and proportionate volume of tasks (work load) to each process according to its strength/capacity. ANU-SOAM can theoretically refine the performance advantage provided by HMPI using ANU-SOAM's scheduling and load balancing features.

\subsubsection{MPI PCM Library}

According to [100], "malleability is the ability to dynamically change the data size and number of computational entities in an application". Using the correct level of granularity is the key to ensure best performance of applications, especially scientific applications. Too coarse or too fine task granularity may result in negative performance of applications. Both [101] and [100] discuss a PCM (Process Checkpointing and Migration) library that will help MPI applications to dynamically reconfigure their task size to the optimum during runtime. This is achieved by saving and restoring the current state of processes and using that information to split or merge those processes at checkpoints to avail a malleable property to MPI applications. PCM uses MPI wrapper classes PCM_Store, PCM_Load, PCM_Merge and PCM_Status for this. There is also a reconfiguration strategy for MPI applications at runtime but it is a bit different from what is envisaged in EasyGrid. In EasyGrid, the reconfiguration is done with the help of scheduling policies according to the runtime information gathered from compute nodes. PCM reconfigures the granularity of message size by creating or merging computing processes.

Attempts to make MPI malleable help to deal with gramularity by allowing the 
application to change its task (data) size dynamically. However if the application is bound by its logic to have finer grained task data, in PCM programming approach of split or merge, that interdependency may compel the application task data not to be malleable beyond certain level. The ANU-SOAM Data Service addresses this issue to some extent by empowering the application programmer to reduce the interdependency of tasks by allowing the data to be modified by SIs (without reflecting those changes for a generation of tasks, with the deferred put functionality) and enabling this updated data to be used for the next generation of tasks (using sync functionality). This approach is theoretically capable of reducing interdependency between task data elements.

\subsection{Shared Memory Paradigms}

A possible solution to deal with finer grained and communication intensive applications is to provide a data fabric around the client and compute nodes of the SOA model. The data fabric refers to memory system where data can be stored, identified, accessed and may be modified with the help of an indexing service. Generally a data fabric works with the concept of shared memory where compute nodes can keep session specific data in their local memory and can update and modify those data to a shared memory system. This data fabric shall ensure data consistency and integrity. There are tools like OpenMP and Global Arrays to work with SOA parallel applications to achieve these goals, which shares many characteristics of ANU-SOAM Data Service.

\subsubsection{OpenMP}

OpenMP is a portable, scalable API that gives programmers a simple and flexible interface for developing parallel applications [102][103]. The OpenMP API consists mainly of compiler directives, runtime library routines and environment variables. It is based on the creation of multiple threads in a shared address space using a fork-join model. These threads share common data by default but can also have private data [29].

In the scope of OpenMP specification document [104], the architects of OpenMP admit that OpenMP enables only user-directed explicit parallelization. This property gives full control over parallelization for the programmer but adversely affects the programmability of OpenMP applications. Moreover it is reported in [29] that OpenMP can deliver good parallel performance for smaller number of processors 
but may not be for larger number of processors. The directives of CSIRO Advanced Scientific Computing Lab also supports the above observation in relation to the use of OpenMP for parallel scientific computing [105].

However, efforts are made to cluster-enable OpenMP, based on software Distributed Shared Memory (sDSM) systems like Intel Cluster OpenMP (CLOMP). [106] suggests region based prefetch techniques for the performance improvement of sDSMs. [107] suggests a method of predicting the performance of CLOMP based on the number and types of page faults encountered in the process. This helps application programmers to develop codes that may reduce the number of page fault interrupts because page fault interrupts involve the non-trivial movement of large data between nodes in CLOMP. Commercial DSM products like ScaleMP also claim to scale well on multiple nodes [108]. However, cluster-enabled OpenMP suffers from scalability issues as number of nodes increase [107].

There are a also efforts to mix OpenMP with MPI to produce a hybrid systems which could produce more efficient and scalable shared memory parallel systems [109]. In these hybrid systems, optimization done by the programmers give better results than the automatic loop parallelisation provided by OpenMP, reports [110]. These approaches rely mostly on the programmer practises and performance tuning to produce efficiency, which adversely affects the programmability of OpenMP.

\subsubsection{Grlobal Arrays}

The Global Array (GA) toolkit is a library for writing parallel programs that use large arrays distributed across processing nodes and was developed at the Environmental Molecular Sciences Laboratory at Pacific Northwest National Laboratory [111]. In 1994, Jaroslaw Nieplocha et.al suggested that GA could "provide a portable interface through which each process in a MIMD (Multiple Instruction stream, Multiple Data stream) parallel program can independently, asynchronously, and efficiently access logical blocks of physically distributed matrices, with no need for explicit cooperation by other processes" [112].

GA offers two types of operations: collective operations and local operations. Collective operations require participation and synchronization of all processes. They include array creation, copying, and destruction. Local operations may be invoked independently by all processes. They include local access to array elements, and fetching and storing data to and from remote locations. Contrary to MPI, GA does not require cooperation between the sender and receiver to transfer 
data [113][114] but is compatible with MPI [111]. In Global Arrays, the programmer is free to use both the shared-memory and message-passing paradigms in the same program, and to take advantage of existing message-passing software libraries. [115] discusses PERSISTENT and PERSISTENT_RETURN modes of data transfer in GA which avoid unnecessary data transfers within a grid environment and supports asynchronous, coarse-grained parallel tasking (It was reported in my previous paper [116]).

GA distributes the data among compute nodes and provides an addressing system to represent them as a single logical block. GA can query where the data is, before starting a computation. As such it is also useful for data intensive computations, when data affinity can be used to direct computations to particular node(s) which needs specific data[113]. GA allows global arrays to be updated and accessed using get, put functionalities. However, GA put is not a deferred operations as in the Data Service extension of ANU-SOAM. GA put update data instantly in a sequential order. Moreover GA sync is only a barrier for all GA operations[114] and does not support the concept of generations of tasks as implemented in ANU-SOAM. GA does not provide native-support to handle heterogeneous environments, unless the programmer puts in place specific load balancing techniques to address it [117].

\subsection{Distributed File System Services}

In order to help data intensive applications, some data grids [118, 9, 19] make use of Distributed File Systems (DFS) like Google File System (GFS) [119] and Hadoop Distributed File System (HDFS) [120]. Their aim is to provide a file system to hold large volume of input data (to the degree of terabytes or petabytes) in a distributed commodity hardware environment by breaking them into smaller chunks and distributing them or even replicating them to support parallelization of an application. In those cases, DFSs serve the purpose of providing a distributed data space using full or partial replication of huge chunks of input data for computations. This approach is neither meant nor effective for computation intensive applications where tasks are inter-independent to each other within the same generation of tasks [121]. This is different to the Data Service extension envisaged in ANU-SOAM which primarily aims to provide a data space which can be updated and synchronized after each generation of tasks throughout the life of an application. 


\subsubsection{Google File System}

The GFS is a proprietary distributed file system developed by Google Inc. It aims to provide reliable access to data distributed along commodity hardware cluster nodes. The design of GFS has been driven by the fact that a component failure in a large commodity cluster is the norm rather than an exception. Therefore the GFS design give more importance to fault tolerance and automatic recovery[119]. GFS is designed to handle big files, because the major intent of GFS was to handle Google's search engine services. To simplify the design GFS assumes that the files once written are only read and often only sequentially, but they allow files to be appended with new data. The architecture of GFS consist of a single master and several chunkservers which are accessed by multiple clients [119].

\subsubsection{Hadoop Distributed File System}

The HDFS, which is also known as Hadoop Core, is an open source distributed file system designed to run on commodity hardware [120]. The main assumptions and objectives of HDFS resembles that of GFS. One of the main difference is that HDFS is designed more for batch processing rather than interactive use by users. That is, the emphasis is on high throughput of data access rather than low latency of data access. Like GFS, HDFS assumes to handle large data sets and anticipate non-trivial probability of component failure. It is based on a write-once-read-many access model for files. That is, it assumes a file, once created, written and closed, need not be changed. Unlike GFS, appending-writes are not incorporated in the present model of HDFS. HDFS has a master-slave architecture with a single Name Node and a number of Data Nodes [120].

\subsection{Chapter Conclusions}

Message passing systems, especially MPI, is considered to be the default tool for high performance scientific computing. There is large degree of complexity involved in programming MPI applications, which is beyond the scope of average scientists or researchers. There are no built in mechanisms to make MPI system-aware and it is found to be agnostic of the underlying systems especially in heterogeneous environments. This research work tries to overcome these limitations by adopting a SOA model with convenient APIs for scientific parallel computing with suitable load balancing mechanisms associated with it. 
The proposed Data Service extension is designed to provide a memory space for client and service instances to be accessed, modified and synchronized by tasks. It can be accessed using a global addressing system, similar to that of Global Arrays. The add, get, update, sync functions provided by many shared memory systems - including Global Arrays - are perfected in the Data Service extension of ANU-SOAM, for example with a deferred put as explained in section 4.1.6. The primary objective of the ANU-SOAM Data Service is to facilitate computation by providing an updateable memory skin around client and service instances rather than to provide large storage for data-intensive applications. This is property different to that envisaged in DFS services. 


\section{Chapter 4}

\section{Design and Implementation}

A middleware - ANU-SOAM - in the mould of SOA was designed and implemented to actualize an efficient high performance computing paradigm for medium grained scientific applications by keeping the core characteristics of programmability, scalability and efficiency under heterogeneous conditions.

The discussions on section 2.4 suggested that the architecture of Platform Symphony is suited to retain SOA characteristics. It was also noted that its API is convenient enough to ensure programmability and it is already in use in enterprise and financial circles with satisfactory ease of programming [6]. It was decided to use Platform Symphony architecture and to adopt its APIs to ensure these features to ANU-SOAM. This decision also helps already existing Platform Symphony applications to be easily ported to ANU-SOAM. In order to deal with scalability and high performance issues of Platform Symphony, a Data Service extension was conceived. It is also intended to reduce communications costs and to improve its performance. Proper load balancing and scheduling functionalities are added to ANU-SOAM to perform efficiently under heterogeneous conditions.

\subsection{Architecture}

Following the general SOA architecture as discussed in section 2.2.1, ANU-SOAM is designed to have 3 major modules - a client, service and resource manager. A Data Service extension is intended to improve performance and scalability of ANU-SOAM (Refer Figure 4.1). 


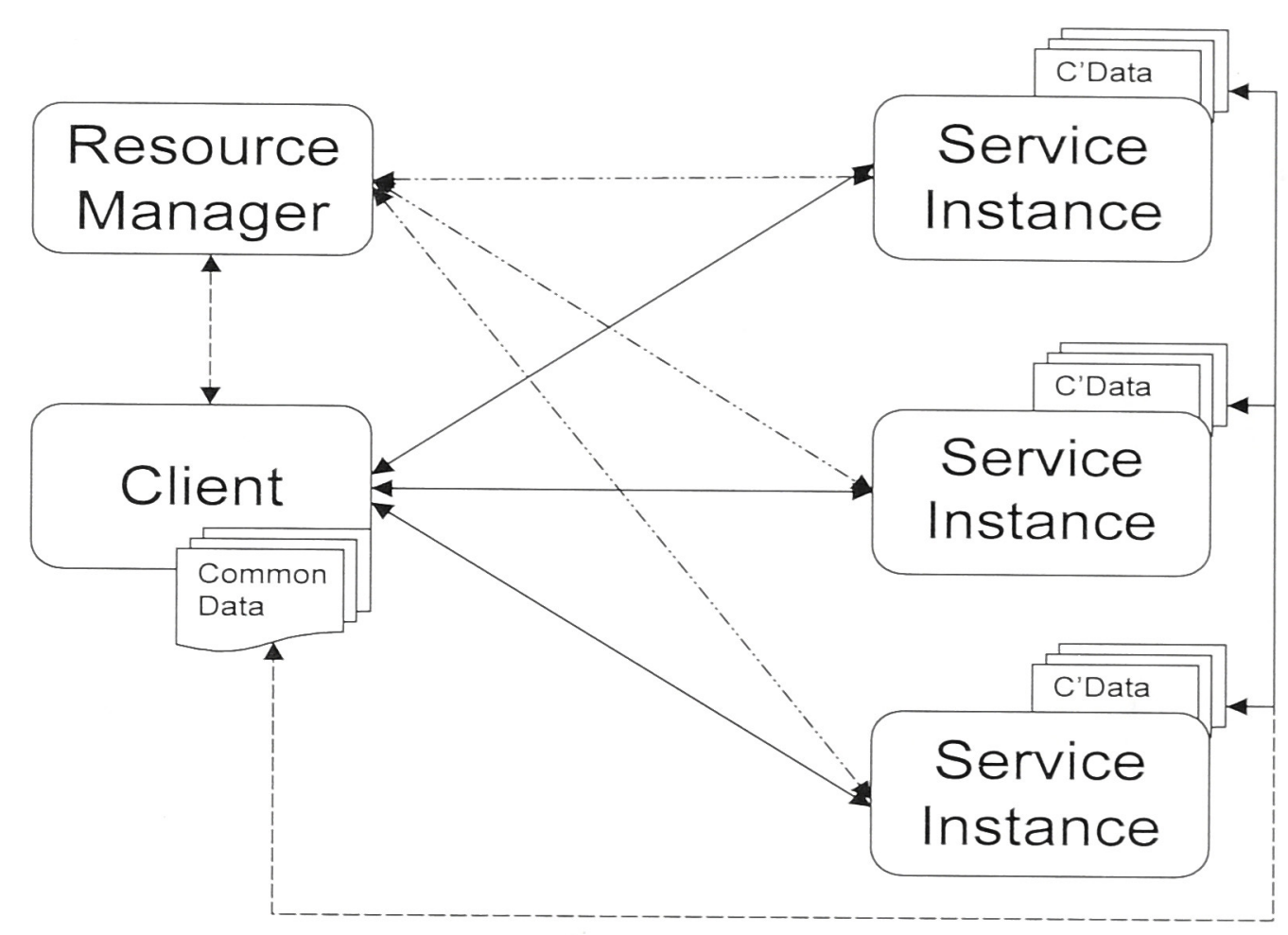

Figure 4.1: ANU-SOAM Architecture

\subsubsection{Design Requirements}

The client module shall initiate the client process and run on the hostnode and the service module shall initiate service processes (Service Instances) and run on compute nodes. The underlying complex work of initialising client and service instances, creating sessions, serializing and deserializing the messages and sending and receiving tasks shall be done by client and service processes supported by ANU-SOAM client and service libraries. The resource manager shall take care of dynamic load balancing and data affinity policies. ANU-SOAM will support only a single application at a time, because it is only an experimental version and therefore does not provide an application registry service as seen in Platform Symphony. ANU-SOAM application programmers shall be able to develop client and service application codes using its API (refer to Appendix A) to express the application logic and compile and deploy them to the client and compute nodes. Application programmers shall be able to make use of the Data Service using add, get, put and sync functionalities.

MPI as Communication Backbone As mentioned in section 2.4.2, the performance of Platform Symphony for scientific applications was too slow to be seriously considered for high performance outcomes. It was also reported in article [89] using experiments with Platform Symphony Matrix Vector Multiplier applications. The investigation of the source code of Platform Symphony (which 


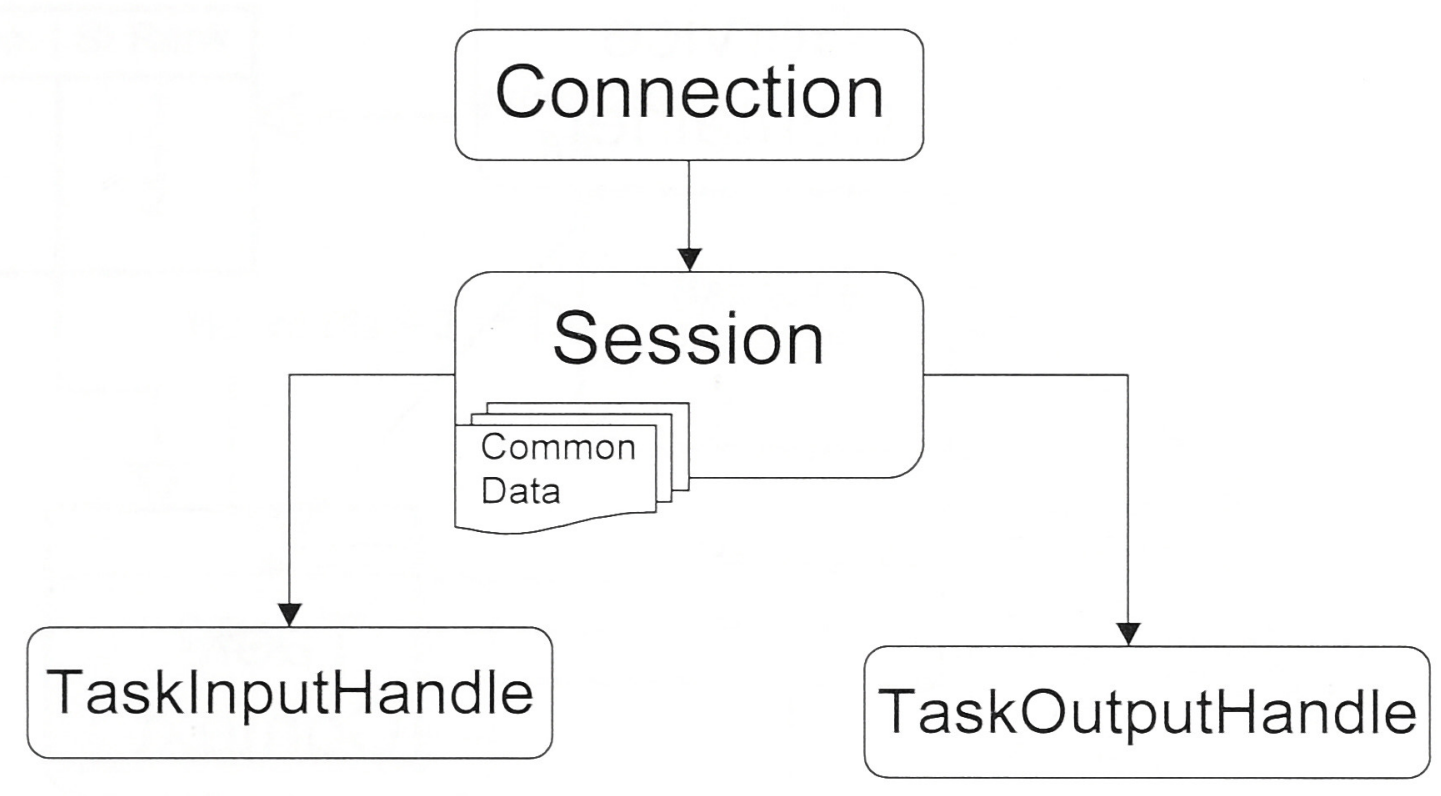

Figure 4.2: Class Diagram: Client Module

was received as part of a formal agreement between Platform Computing and ANU) found that it uses Adaptive Communication Environment (ACE) - "an object-oriented toolkit that implements fundamental design patterns for communication software" [122] - as its communication regime. It makes sense for Platform Computing to use ACE because it extends modularity and extensibility of the object-oriented programming model to its developers in the development and maintenance of Platform Symphony as an enterprise product. However this approach has not produced the expected high performance outcomes we expect in scientific computing. On the other hand, MPI is seen as the natural choice for parallel high performance application development [90][123]. On this presumption, it was decided that ANU-SOAM should be developed using MPI as its communication system.

\subsubsection{Client Module}

At the client, the process starts with MPI process creation by initializing SoamFactory class (refer source code snippet at Appendix B.1). The service processes are spawned dynamically using MPI_Comm_spawn technique available in MPIII. It provides an inter-communicator between client and the spawned service processes which enables the client and services to communicate with each other. A connection is established as an inter-communicator handle between client and service processes. This connection instance is made persistent throughout the life of the application as a session (refer source code snippet at Appendix B.2). A session 


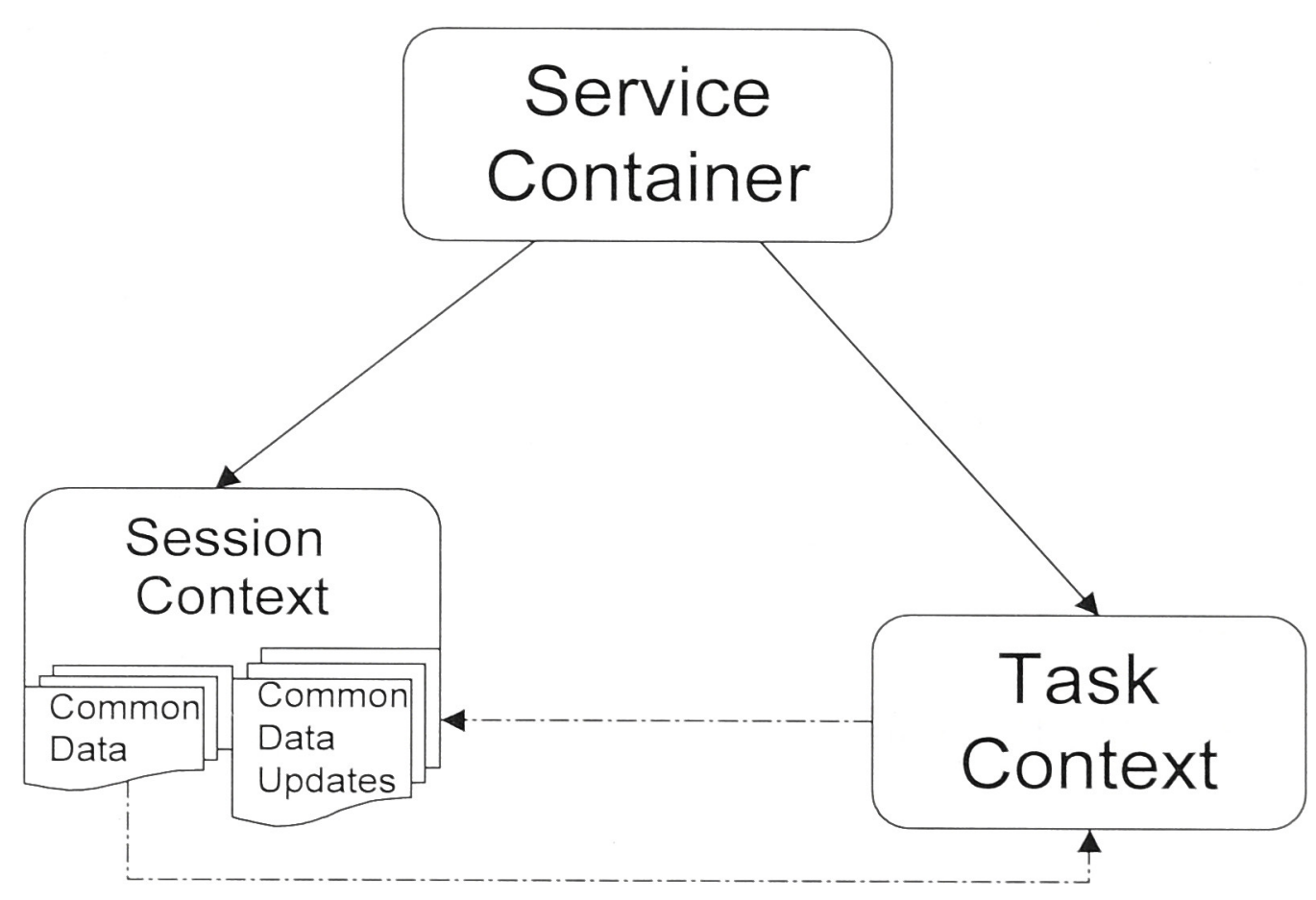

Figure 4.3: Class Diagram: Service Module

is defined by session creation attributes which are basically MPI_Comm_spawn attributes. There is provision to choose a default setting for these session creation attributes. The API also gives an option to modify some of those attributes after default settings are called (e.g: service name, maximum number of service instances, service executable path, etc). Task input and output handles send and receive task messages within a session. The client class diagram is given in Figure 4.2 .

\subsubsection{Service Module}

The service processes are created dynamically by the client using the MPI spawn function and are individually initiated by separate MPI_Init calls. The service processes then accept the parent communicator and merge to create an intercommunicator with the client to establish connection. On session creation, a service process is set in an infinite loop to receive tasks from client, till client completes that session (refer source code snippet B.6). At service nodes tasks are processed on a First In First Out (FIFO) basis. The service class diagram is given in Figure 4.3.

\subsubsection{Resource Manager Module}

ANU-SOAM implements static load balancing techniques with its Resource Manager (RM) module. The RM is integrated with the client process for design con- 


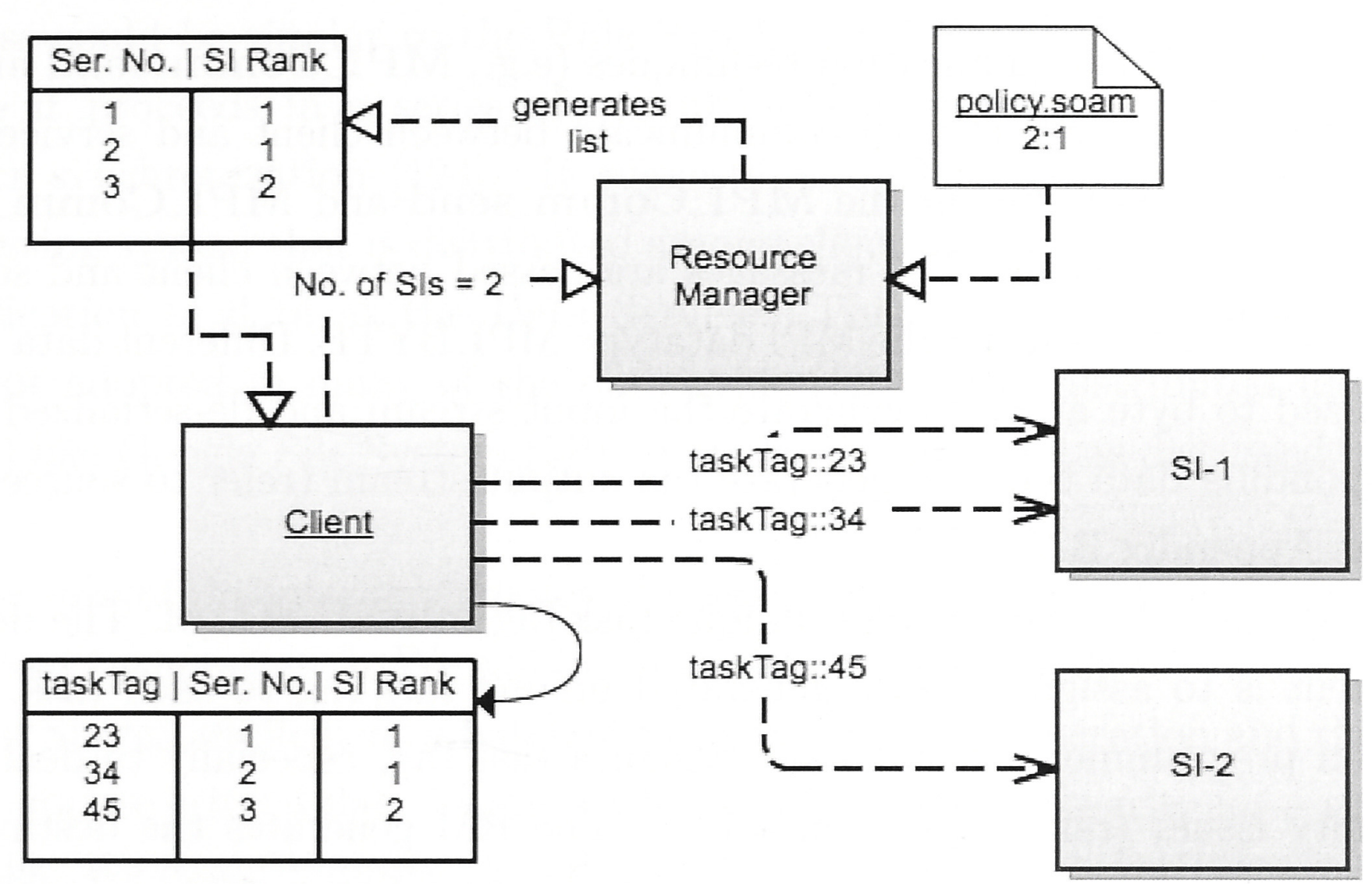

Figure 4.4: Load Balancing Using 'weighted round-robin'

venience. RM is to be supplied with the knowledge of available resource nodes - that is their hostnames and relative processing powers. The application programmer can choose resource allocation policies like, random, round-robin or weighted round-robin to distribute tasks among SIs (a compute node can host more than one SI). The default setting at RM is to send tasks to each of these nodes in a round-robin fashion. When load balancing is activated using weighted round-robin policy, the RM reads relative weights of these nodes from a file called 'policy.soam'. The RM gets the maximum number of SIs from the client application code. The RM generates a list with task (serial) numbers against that of SI (MPI) rank number, according to the weighted round-robin policy. This list is kept by the RM for the life of a session and made available to the client. The client self generates taskTags in case of fully replicated Common Data and associate them with the list from the RM. In case of non-replicated Common Data, the application programmer can designate taskTag to tasks to ensure data affinity and consistency (refer to section 4.1.6). A sample load balancing and scheduling dynamism is pictorially given in Figure 4.4. 


\subsubsection{Messages, Tasks and Tasklets}

Point-to-point MPI communication techniques (e.g., MPI_Comm_send and MPI_Comm_recv) are used to communicate between client and service processes. The message buffer in the MPI_Comm_send and MPI_Comm_recv are used as task messages. The messages are passed between client and service processes as byte arrays using the MPI datatype MPI_BYTE. Different data types are serialized to byte array to generate the input stream and de-serialized back to corresponding data types to generate the output stream (refer to source code snippet at Appendix B.3).

The tasks are identified using a unique taskTag in ANU-SOAM. The default arrangement is to assign an auto-generated unique taskTag to each task. The application programmer can manually assign a taskTag, especially to deal with data affinity issues (refer to section 4.1.6). The RM generates the destination node rank of a task using load balancing policy assigned to it. This arrangement enables ANU-SOAM to sent multiple tasks within a session to any SI. Once all input tasks are sent, the client waits for the output tasks from the service. On arrival of output tasks, the messages are stored into a linked list. Once all the expected outputs are back, the messages can be enumerated and populated onto client output message buffers to process the output data (refer to source code snippet at Appendix B.5).

A task may need to process different types of computations/procedures within the same task. In order to facilitate these different operations, tasklet concept is introduced in ANU-SOAM. Each tasklet within a task can be uniquely identified using a taskletTag (an integer) which can be associated with a task using TaskSubmissionAttributes object (refer to section 4.1.6). Each tasklet algorithm can separately be implemented using a switch at the onInvoke method at the service code.

\subsubsection{Data Service Extension}

The aim of the Data Service is to reduce direct communication between the host (client) and compute (service) nodes by providing a service around the client and compute nodes to store and manipulate data with the ease of a global addressing system. Within this Data Service, compute nodes can keep session specific data in their local memory and can get, update and modify that data, on a local level or on a global level. The global updates can be synchronized incrementally at some barrier points, based on the application's algorithm. The client process 
will be able to fetch this modified and synchronized data at any time. This process could be similar to the Bulk Synchronous Parallel computing technique where it proceeds in a series of concurrent computation, communication and barrier synchronization [124]. It suggests a local memory space and a global addressing system that is distributed among client and compute nodes to support modification of data at the Data Service. This is different to the data fabic concept adopted in many of the data grids [118] using distributed file systems (DFS) like Google File System [119] or Hadoop Core [120] as discussed in section 3.3 .

Because of the interdependency of tasks, many scientific applications progress in phases (generations). We can improve the efficiency of those scientific applications if SIs can implement a batch of tasks in the same generation and those tasks could update critical data locally without losing logical integrity for a generation of tasks. We could improve the efficiency further if SIs can start new generation of tasks on their own without communicating back to the client. These features are possible with the help of an extended Data Service.

\section{Common Data}

The ANU-SOAM Data Service is an extension of Common Data function within Platform Symphony. A data array initiated at the Data Service is called Common Data. In Platform Symphony, Common Data arrays cannot be modified. They remain as read-only data at SIs and are a part of SessionCreationAttributes. The Platform Symphony Common Data is persistent throughout the session and it reduces the need to send data common to all generations as separate task messages for all generations and thereby reduces data transfer overheads.

While retaining the Common Data function of Platform Symphony within SessionCreationAttributes, ANU-SOAM provides a Data Service which can hold Common Data that can be modified by tasks at SIs. This new Common Data with its added functionalities became ANU-SOAM's Data Service extension with new set of APIs (refer to Appendix A). This Common Data can either be initiated from the client process and then communicated/distributed to the SIs or can be just initiated at the SIs. It is then accessible at onSessionEnter or at onInvoke functions at service code as described in section 4.2.1. The Data Service can have many Common Data arrays identified by unique names. Refer to Figure 4.1 for a pictorial representation of the proposed architecture. 
Fully Replicated Common Data Some applications may require the whole data to be made available to all SIs for all generations of tasks (for example, NBS - refer to section 5.1). In that case the Common Data can be fully replicated to all SIs for the life of a session. Fully Replicated Common Data implements all four major functions - add, get, put and sync - and other variant functions.

Non-Replicated Common Data In some other cases, application logic may not suggest all SIs to have all the data, but only part(s) of it to do parallel jobs (for example, HTS - refer to section: 5.2 and CGS - refer to section 5.3). Full replication of Common Data can put strain on memory at compute nodes and reduce memory scalability, if the data is large. If the whole data is not needed for all the tasks, those partial data blocks (for example, sub-matrices) that are needed for the respective tasks can be made available to those tasks using non-replicated Common Data functionality. It will help reduce memory needs of the application by storing only the required part of the data in the Data Service for a particular task. The concept of non-replicated Common Data was first envisaged as Local Data and reported in [116], which has now been integrated into Common Data by providing a extended interface to the Data Service for programming convenience.

\section{Enabling Data Affinity}

In order to keep data integrity and logical consistency over generations of tasks, data affinity principles are applied to non-replicated Common Data. Non-replicated data in the Data Service is associated with a task, but not to an SI. This helps application programmer to ignore how the non-replicated Common Data is distributed to SIs, but to look logically on how it is linked to a task. That is, the application programmer need not know which SI or the compute node holds a particular partial data.

This is achieved by introducing a taskTag (an integer) attribute, which can be linked to a non-replicated Common Data and to tasks. When a non-replicated Common Data is declared, a taskTag can be associated with it by introducing it as a Common Data attribute as below:

addCommonData(name, dataType, numberOfElements, taskTag);

Now the application programmer can choose any task to use/reach that data by linking the same taskTag as a taskSubmissionAttribute like: 

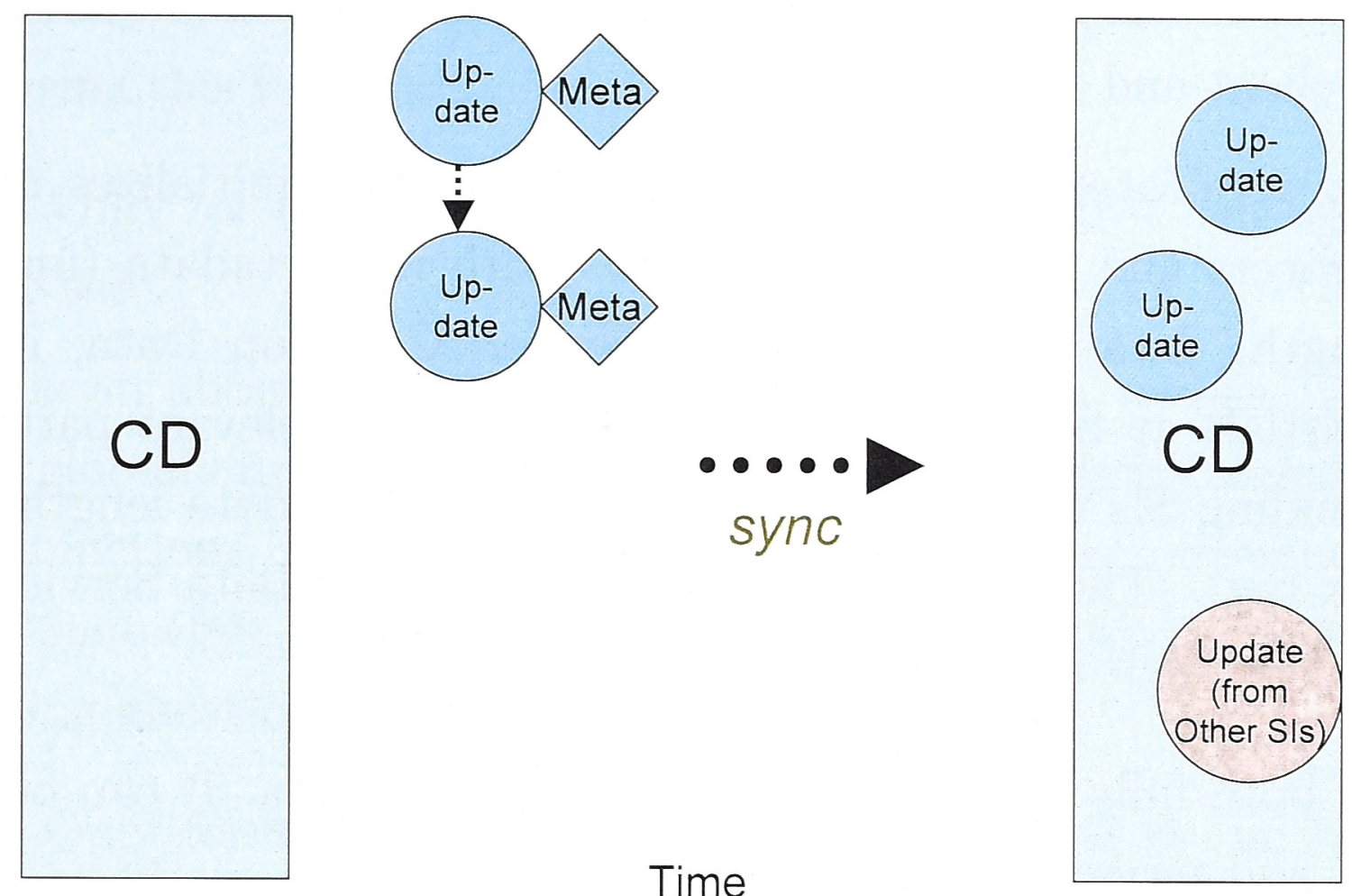

Figure 4.5: Deferred put Action in a SI (for Data Consistency)

$$
\begin{gathered}
\text { TaskSubmissionAttributes attrTask; } \\
\text { attrTask.setTaskTag(taskTag); } \\
\text { attrTask.setTaskletTag(taskletTag); } \\
\text { sendTaskInput(attrTask); - }
\end{gathered}
$$

With these two steps the non-replicated Common Data will be linked to a task. We can have more than one tasks that uses the same data by associating them with the same taskTag. Since this data is not replicated to all the SIs, the updates to non-replicated Common Data need not and cannot be synchronized globally.

\section{Common Data Functions}

Common Data has four major functions - add, get, put and sync-along with other variant functions like put_i, put_b, iSync and $i$ Get using APIs given in Appendix A.1, A.2. MPI collective operation -MPI_Comm_bcast - is used to distribute data from client to SIs in the add function. The sync and iSync functions are implemented using collective call - MPI_Comm_allgatherv - among the SIs using a separate communicator (refer to pseudo code snippet at Appendix B.7). Based on the replication policy, there are two types of Common Data - fully replicated Common Data and non-replicated Common Data. 
- add: For both fully and non-replicated Common Data and it can be used both at client and service sides.

For fully replicated Common Data, this function initializes data at the client process and distributes to all SIs with its metadata (unique name, data length, data type). In non-replicated Common Data, it initializes data (partial) at the client process and transfers relevant partial data to corresponding SIs with its metadata (unique name, data length, data type and task tag). These data arrays are listed in the Data Service, uniquely identified by their names.

- get: For both fully and non-replicated Common Data. It can be used both at client and service sides.

A Common Data array on the Data Service list is accessed and the data is copied to the memory space pointed by the application code at the client process or at SIs.

- put: Only for fully replicated Common Data and it can be used only at service side.

This function allows updates to fully replicated Common Data according to the application logic at SIs. This is a deferred action.

Keeping Data Consistency in put: In order to keep consistency of Common Data in Data Service for all tasks within a generation, put is implemented as a deferred action. That is, the updates to Common Data proposed by tasks are not written instantaneously to the Data Service. If that is done, other tasks within the same generation will be accessing corrupted data at Data Service for that generation. The updates are put into a list along with its metadata (all information regarding that update). These updates are retrieved from the list and then synchronized (sync) using the informations at the metadata at the end of all tasks in a generation and only at the request of the client process. Please refer to Figure 4.5 for the pictorial understanding of this process.

- put_i: For both fully and non-replicated Common Data and it can be used only at service side.

put_ $i$ is an abbreviation of put_instantaneous. It is a variant function of put which is implemented as an instantaneous update of the local copy of Common Data at the Data Service associated with each SI. This put cannot 
be globally synchronized. Non-replicated Common Data can be modified, only using this function.

- put_b: Only for fully replicated Common Data and it can be used only at service side.

put_b is an abbreviation of put_boundaries. It is a variant function of put which globally updates only the boundaries of the data block referred in the put_b function. The updated inner elements (if any) are ignored during a global sync after put_b. This is introduced to efficiently deal with algorithms involving ghost cells (for example, HTS).

- sync: Only for fully replicated Common Data and it can be called only from client side.

It synchronizes all present updates to Common Data among all SIs and then updates it back to the Data Service at the client process. The position of these updates in the Common Data are read from the metadata associated with them in the update list (generated by put). In case of put_b updates, the inner elements of the updated two dimensional data block is ignored while synchronizing.

- iSync: Only for fully replicated Common Data and it can be used only at service side.

It is a variant function of sync which synchronises Common Data among all SIs but is not updated back to the Data Service at the client process.

- iGet: For both fully and non-replicated Common Data and it can be used only at client side.

It is a variant function of get and goes hand in hand with the iSync call. It is used to get the latest synchronized data to the Data Service at the client process from SIs. It first synchronizes Common Data at the client process with that of the SIs and then make it available to the client process. It has to be used if iSync is used for synchronization of Common Data in previous iterations.

\section{Common Data Indexing}

Common Data can be a single dimensional or two dimensional array. In case of a single dimensional array it follows the $\mathrm{C}++$ indexing convention which starts 


\begin{tabular}{|l|l|l|l|l|l|l|l|}
\hline$(0,0)$ & $(0,1)$ & $(0,2)$ & $(0,3)$ & $(0,4)$ & $(0,5)$ & $(0,6)$ & $(0,7)$ \\
\hline$(1,0)$ & $(1,1)$ & $(1,2)$ & $(1,3)$ & $(1,4)$ & $(1,5)$ & $(1,6)$ & $(1,7)$ \\
\hline$(2,0)$ & $(2,1)$ & $(2,2)$ & $(2,3)$ & $(2,4)$ & $(2,5)$ & $(2,6)$ & $(2,7)$ \\
\hline$(3,0)$ & $(3,1)$ & $(3,2)$ & $(3,3)$ & $(3,4)$ & $(3,5)$ & $(3,6)$ & $(3,7)$ \\
\hline$(4,0)$ & $(4,1)$ & $(4,2)$ & $(4,3)$ & $(4,4)$ & $(4,5)$ & $(4,6)$ & $(4,7)$ \\
\hline$(5,0)$ & $(5,1)$ & $(5,2)$ & $(5,3)$ & $(5,4)$ & $(5,5)$ & $(5,6)$ & $(5,7)$ \\
\hline$(6,0)$ & $(6,1)$ & $(6,2)$ & $(6,3)$ & $(6,4)$ & $(6,5)$ & $(6,6)$ & $(6,7)$ \\
\hline$(7,0)$ & $(7,1)$ & $(7,2)$ & $(7,3)$ & $(7,4)$ & $(7,5)$ & $(7,6)$ & $(7,7)$ \\
\hline
\end{tabular}

Figure 4.6: Common Data 2D Indexing

from 0. For the convenience of application programmers, ANU-SOAM Common Data can also be assigned as a two dimensional array. This service is not available in Platform Symphony. ANU-SOAM follows indexing convention used by Global Arrays[114] as shown in Figure 4.6. There are computational costs involved in defining two dimensional Common Data array rather than single dimensional Common Data in the ANU-SOAM implementation. This limitation arises from the fact that MPI communications (like Recv/Send) can handle only one dimensional arrays, which is the communication tool of ANU-SOAM. So every two dimensional $\mathrm{C}++$ array declared as Common Data in the Data Service has to be converted to a one dimensional $\mathrm{C}++$ array to communicate between client and SIs or among SIs. This will have performance implications in the Common Data loading time (refer to section 6.1.2), Common Data accessing time (refer to section 6.1.2) and in the execution of sync function among the SIs.

Section 4.2.2 describes how programming models will change with the introduction of the Data Service from that of conventional SOA architecture. The Data Service will enable programmers to develop scientific applications with innovative algorithms. 


\subsection{Application Development}

With the help of ANU-SOAM APIs and configuration files programmers can develop client, service and message codes for ANU-SOAM applications in $\mathrm{C}++$ language. There are basically two approaches to do this - firstly, the conventional SOA approach and secondly, the advanced ANU-SOAM approach.

\subsubsection{Conventional SOA Approach}

The conventional SOA approach involves developing applications as interactions between client and service instances without the help of a Data Service. This method is used in Platform Symphony application development [125] and the client and service codes are developed with the help of APIs as described in [126]. The message class(es) can be defined outside client and service codes which are also supported by a set of APIs.

Since ANU-SOAM implements most of the Platform Symphony APIs (refer to Appendix A) and its architecture, the conventional style of programming is retained in ANU-SOAM similar to that of Platform Symphony. The conventional SOA programming steps are shown in the sequence diagram 4.7. The basic steps in developing a Platform Symphony client and service are given in [125] and are reproduced here with modifications to emphasis ANU-SOAM programming style.

\section{Developing a Client}

1. The client calls SoamFactory.initialize() to initialize the API.

2. The client calls SoamFactory.connect() to establish a connection Platform Symphony. In ANU-SOAM it is a stub, because it supports only a single application at a time in the experimental version.

3. The client create a session to group tasks using Connection.createSession(). An API call - SessionCreationAttributes - is used to define the special attributes of that session.

4. The method creates and returns a session object, which acts as a conduit through which the client can send input to its service.

5. The client creates an input message object and invokes Session.sendTaskInput() on the session object. This starts the chain of events that eventually sends the input to the service for processing. 


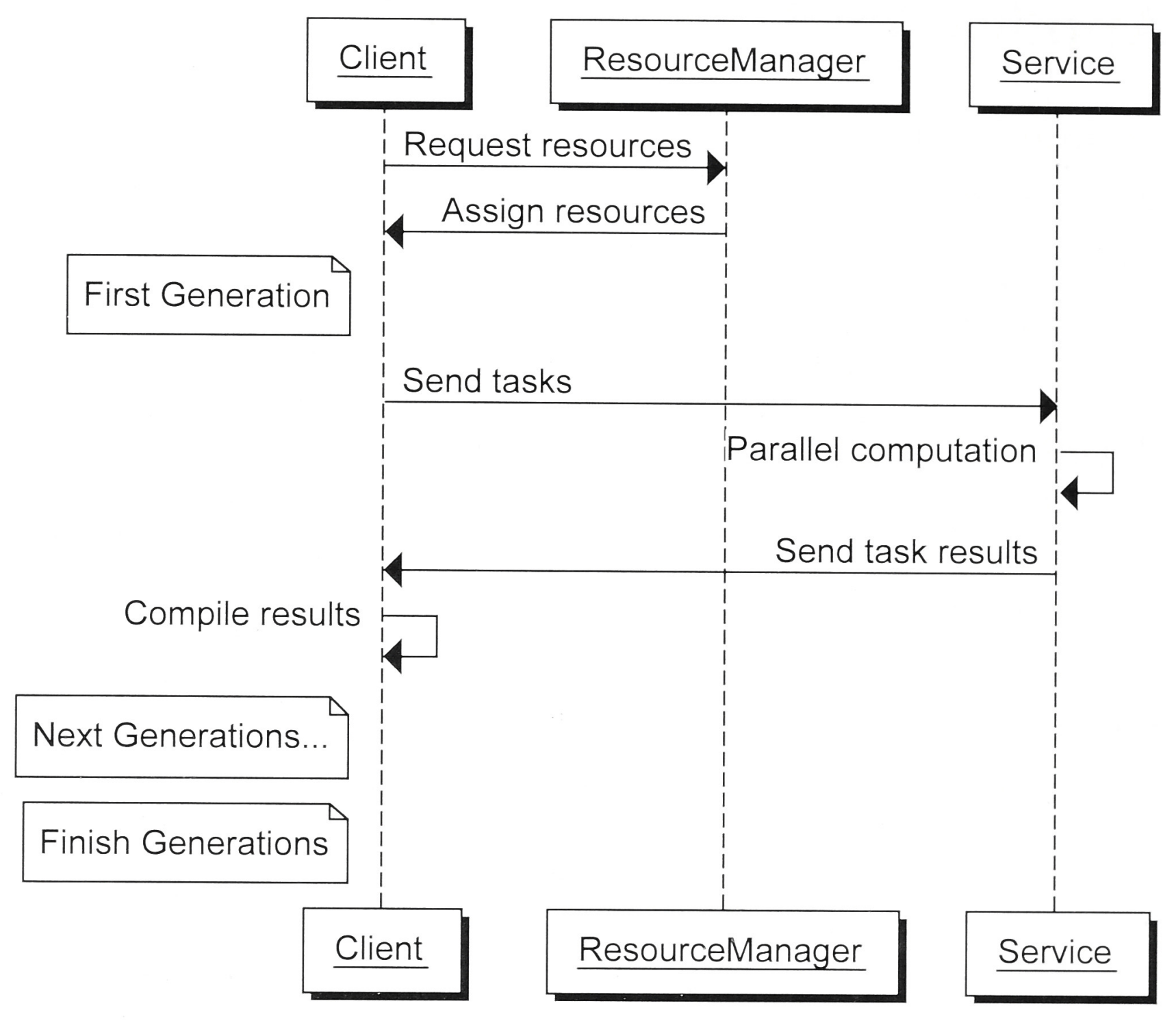

Figure 4.7: Sequence Diagram: Conventional SOA Approach

6. The call to sendTaskInput() causes an OutputStream to be internally created.

7. The session calls onSerialize(...) on the input message and passes the OutputStream to the method.

8. In the onSerialize(...) method, the input message writes itself to the provided OutputStream.

9. The byte array representation of the input message is sent to the middleware.

10. As a result of the initial sendTaskInput(...) call, the middleware returns aTaskInputHandle to the client, which contains an identifier that can help match the input to the output that will later return. At the same time, the middleware also sends the input message to the service for processing and obtains the output from the service invocation.

11. The client calls the Session.fetchTaskOutput(...) method on the session object. 
12. The middleware sends the output back to the client as a result of the fetchTaskOutput() method call.

13. The output is put into an EnumItems object, which is basically a list of outputs.

14. The client iterates over the EnumItems object to inspect each TaskOutputHandle. TheTaskOutputHandle is a container for the output from the Service.

15. Uninitialize with SoamFactory.uninitialize().

Session Creation Attributes: When creating a session, the application programmer can specify the session attributes using the SessionCreationAttributes object in ANU-SOAM:

- ServiceName: The name (char array) of the service is passed on to setSer$\operatorname{viceCmd}(\ldots)$.

- Number Service Instances: The maximum number (integer) of SIs (resources) intended to be used is declared at setNoOfChildren(...).

- Number of Tasks: The number (integer) of tasks to be called for each generation is set at setTaskDataAffinity $(. .$.

- Resource Manager Policy: There are provisions for three resource manager policies (char array): "random", "round-robin" or "weighted-rr" (weighted round robin) and can be set at setRMPolicy $(\ldots)$.

- Default Settings: setDefaultComm() will set default configuration as ServiceName = "service", Number Service Instances $=2$ and Resource Manager Policy = "round-robin".

- Common Data: ANU-SOAM retains Common Data implementation of Platform Symphony as part of SessionCreationAttributes through setCommonData(...) (message object), in addition to the new Common Data implementation within its Data Service. 
Task Creation Attributes: When creating tasks within a session, application programmer can specify the following task attributes using the TaskCreationAttributes object in ANU-SOAM.

- TaskTag: Allows to tag tasks within a generation to be identified with a unique number (int) using setTaskTag(...) call.

- TaskletTag: Allows tasks to have tasklets within them using setTaskletTag(...). It enables tasks to have multiple tasklets with same taskTag but different taskletTags.

- Message: Attaches a message (message object) to a task using setTaskIn$\operatorname{put}(\ldots)$.

\section{Developing a Service}

There are five core methods in the service code as explained below. The following indicates the structure of a service with all methods implemented, in the order that they need to be implemented.

1. onCreateService(): Optional. It is invoked just after it launches the service instance to initialize the service instance. In ANU-SOAM it is a stub.

2. onSessionEnter(): Optional. The middleware invokes onSessionEnter() on first use by a session. Session specific data/operations are assigned/done here.

3. onInvoke(): Required. The middleware invokes onInvoke() to compute a task. This method is called once for every task received at an SI. The method can be called in the same SI multiple times to compute multiple tasks in a session until the SI is assigned to another session. Tasklets are identified by taskletTags within this method.

4. onSessionLeave(): Optional. It is called once when a service instance is unassigned from a session to free up session specific data from memory.

5. onDestroyService(): Optional. It is called once when a service instance is ending its lifecycle to clean up a service. In ANU-SOAM it is a stub. 


\subsubsection{ANU-SOAM Approach}

The Data Service extension and its corresponding API calls will help application programmers to think differently to develop innovative algorithms to make applications faster on ANU-SOAM. The Data Service enables programmers to transfer more of the client code to service side (the compute side) with the help of add, get, put and sync functions of Common Data. This is achieved by enabling successive generations of tasks to be serially executed at the service side by allowing updating of Common Data at Data Service. The results (or updates) at SIs are not communicated back to the client only when necessary, but not after executing each and every tasks. This approach will improve the performance of the application in two ways:

1. by reducing data transfer between the client and service processes and

2. by increasing the apparent granularity of fine to medium grained applications to reach optimum granularity to benefit from parallelizing those application codes.

\section{Use Case}

A use-case for this scenario may be the $\mathrm{N}$ Body simulation of a planetary system. It may not be necessary to get each and every updated velocities and positions of all planets at every small increment of time $(\delta t)$ to generate a simulation. We can afford to get the results at fairly larger intervals, but still get acceptable resolution for simulations.

The sequence diagram shown in Figure 4.8 explains how the Data Service will change the way application programmers would develop new applications and a comparison of it with the conventional SOA programming can be made with the sequence diagram shown in Figure 4.8.

\section{Developing a Client}

Steps 1-4 of sub-section Developing Client at section 4.2.1 remain the same. Before step 5 (That is, before starting to send tasks), Common Data can be added from the client side. Steps 11-14 of section 4.2.1 describes the conventional way of getting tasks results from the service side. These steps will be replaced with sync/iSync/get/iGet in the advanced approach. Instead of getting back the task 


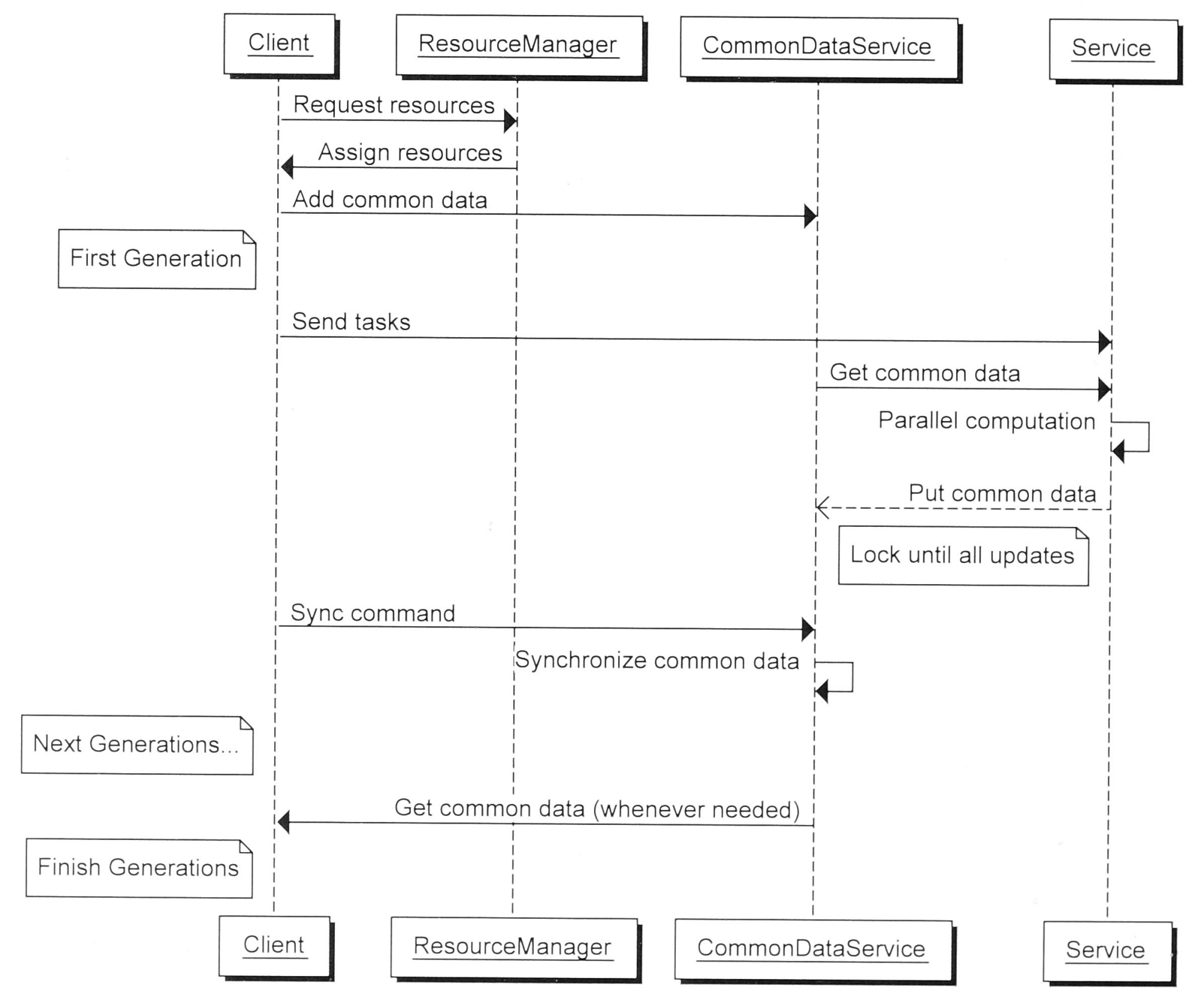

Figure 4.8: Sequence Diagram: Advanced ANU-SOAM Approach 
results, the client sends sync/iSync commands to the service side after each generation of tasks to update respective Common Data at the service side. Finally the client gets the required results by a get/iGet.

\section{Developing a Service}

All the steps described at sub-section Developing a Service at section 4.2.1 can be used in a similar way in the advanced version too. Common Data can be accessed (get) either by onSessionEnter() or by onInvoke(). Service instance specific Common Data can be added at onSessionEnter() of service code. This service instance specific Common Data will be available at onInvoke(), just as the Common Data declared at the client side made available at the service. This Common Data can also be accessed at the client by using iGet command at client side.

At onInvoke(), task messages are received and application logic is implemented. The results can be updated (put) to relevant Common Data by any task. However as explained before (refer to section 4.1.6), these results are not immediately reflected at the Data Service, and data consistency of Common Data for a particular generation of tasks is maintained. Instantaneous update of Common Data at an SI can be done using put_i functionality, if needed. put_b functionality allows global synchronization of the boundaries of a two dimensional Common Data. The other functionalities remain the same in this approach compared to the conventional approach.

\subsection{Application Deployment}

Client application code can be compiled using client and resource manager libraries. Service code is compiled using service libraries. A set of makefiles and scripts are provided to assist these purposes. These executables are deployed to the host and compute nodes respectively.

The client and service application codes can be developed by the application programmer, along with the message class codes. Client application code is compiled using client and resource manager libraries and service code is compiled using service libraries using standard GCC-MPI compilers. A set of makefiles and scripts are provided to assist these purposes. The Service Instance executables have to be deployed manually to all compute nodes. The deployment strategy is explained in Figure 4.9. 


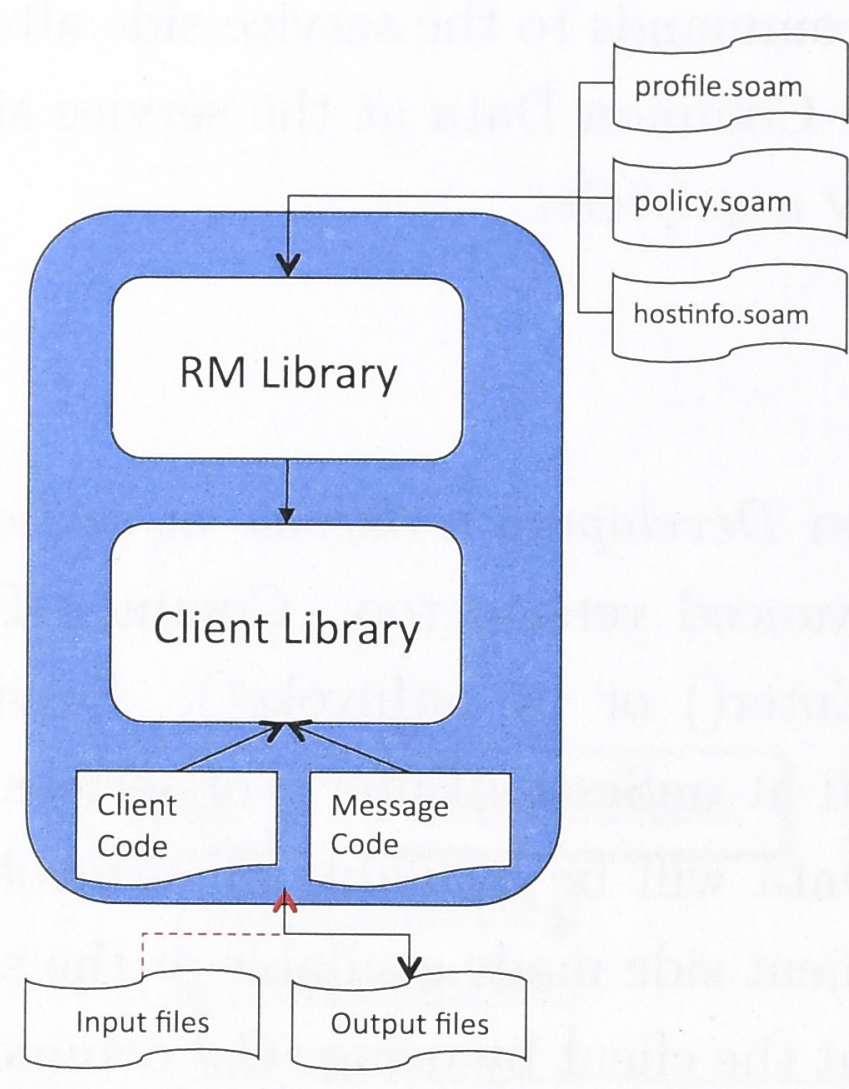

At host node

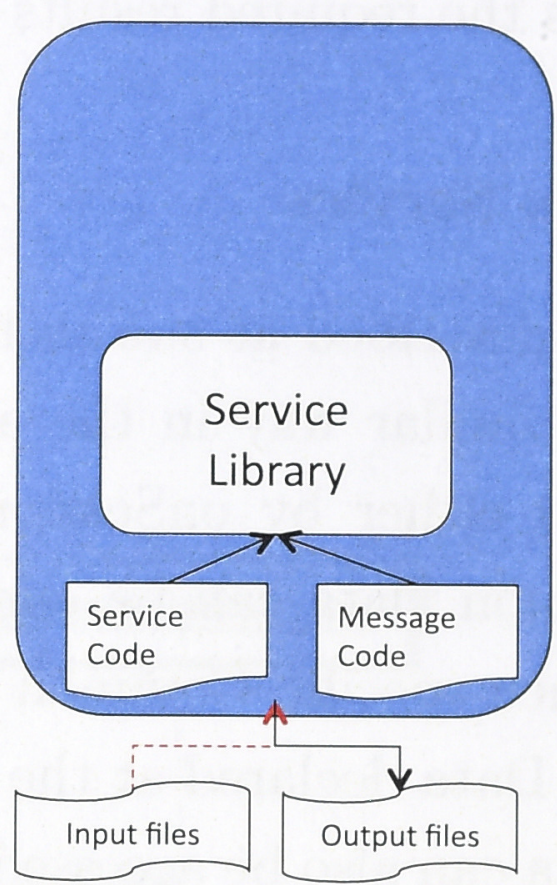

At compute nodes

Figure 4.9: ANU-SOAM Deployment

Using the standard MPI Command Line Interface (CLI), the client process can be initiated. It will run on the host node which implements the client application code. The service instance(s) on each compute node is initiated by the client process, as directed by the RM.

\section{Configuration Files}

There are three configuration files that the Resource Manager will look for. They are:

1. hostinfo: Consists of the hostnames of the resource nodes and the number of processes that can be hosted on each node separated by delimiters, comma and end-of-line respectively.

2. profile.soam: Consists of the path to the service executable.

3. policy.soam: The proportional weight (efficiency) of all compute nodes are provided in this file, separated by the semicolon delimiter. It is used when 'weighted round robin' is the resource management policy. 


\subsection{ANU-SOAM on Cloud}

ANU-SOAM has developed using $\mathrm{C}++$ and MPI with around 20,000 lines of codes and released as an open source product. It has the potential to claim a significant place in the cloud computing stack. It could function as a PaaS for scientific computing and provide a testbed for IBM-Platform Symphony compatible cloud applications. The ANU-SOAM deployment and experiments on a cloud IaaS as described in section 6.3 corroborate these assumptions.

\subsubsection{A PaaS for Scientific Computing}

As shown in Figure 4.10, ANU-SOAM comes under the category of a stand-alone PaaS installation in the cloud stack, like ManjaraSofts Aneka platform [72]. With the current configurations it can be deployed on an IaaS public cloud or a private cloud, which provides compatible machine and network configurations. With its APIs, libraries, deployment scripts and scheduling and load balancing support, it could enable customers deploy scientific applications on a IaaS in a private cloud.

In a cloud environment, the customers are agnostic of the network configurations and its overheads. This is a problem for scientific computing whose tasks are highly interdependent and communication intensive in nature. Lamia Youseff et. al in [64] points out that "ideally, scientific applications in the cloud should have the capability to dynamically adjust their communication-to-computation ratio in order to transparently endure the high variability in the cloud performance". ANU-SOAM is designed to reduce communications between its client-SI and SI-SI modules using its Data Service functions even without demanding a knowledge of it underlying network conditions and therefore better positioned to emerge as a suitable PaaS for scientific applications.

\subsubsection{Enable Real time Applications on Cloud}

At the moment SaaS cloud services are leaning towards supporting batch-processing and analytic applications which are generally embarrassingly parallel in nature. But there are applications that accept run-time inputs which affect further computations. These real time applications are considered to be out of the scope of cloud computing since they demand constant communications between the cloud infrastructure and the client over the Internet or slow networks, which will have negative performance impact on the applications. 


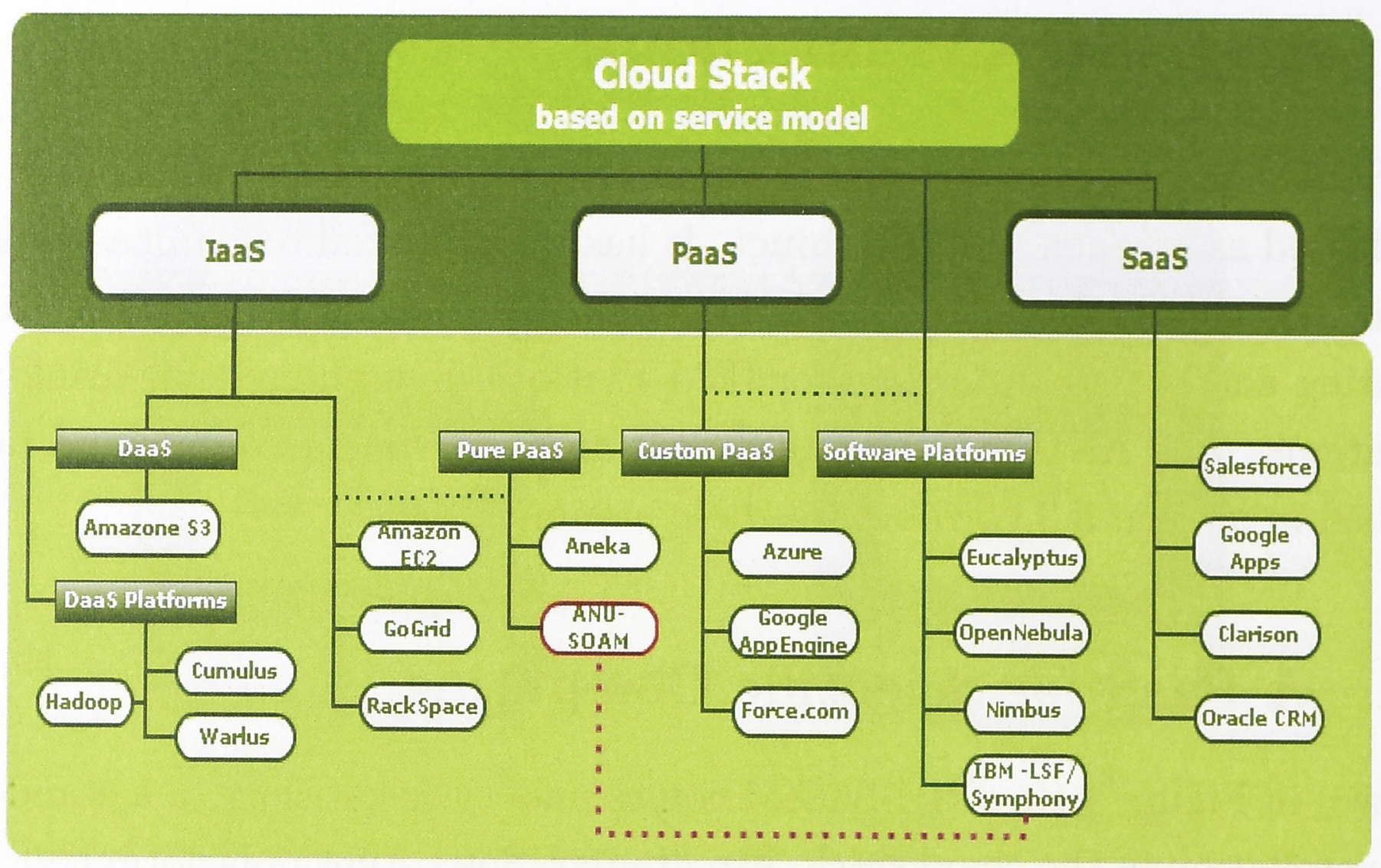

Figure 4.10: ANU-SOAM in the Cloud Computing Stack

A use case for such a scenario would be a real time simulation of N-body problem using cloud resources where new bodies join or leave the system from time to time or the velocity or the position of bodies are influenced by external forces very frequently. Another use case is a system to suggest various traffic routes to a moving vehicle based on its current progression using the vehicle's Geographic Positioning Systems (GPS) as sensors, especially in high performance situations like car racing, using cloud resources.

Even though these use cases are beyond the scope of cloud computing at present, [66] predicts the emergence of such cloud applications for the future which would "likely to have a piece that runs on clients and a piece that runs in the cloud". They further expect that "the cloud piece needs to both scale up rapidly as well as scale down, according to the need and intensity of the scenario." The Data Service extension of ANU-SOAM can enable applications to deal with such scenarios where programmers can develop smart algorithms, which can sits partially on the client side and mostly on the cloud side. The SIs will be communicating to the Data Service rather than to the client to move to the successive generations of tasks and thereby reducing communication overheads in talking to the client over the Internet frequently. 


\subsubsection{A Testbed for IBM-Platform Symphony Compati- ble Cloud Application Development and Integration}

One of the attractions of Eucalyptus cloud software platform is its compatibility with Amazon EC2. Since Eucalyptus implements Amazon APIs, it is used as a on-premise open-source test-bed for applications that are to be deployed commercially in EC2 [65]. ANU-SOAM has similar potential because, it implements Platform Symphony APIs for application development. With the recent acquisition of Platform Computing by IBM to make it part of IBM cloud project, ANU-SOAM can become an open-source on-premise implementation of the IBM cloud suite and contribute to its Smart Planet project worldwide.

\subsection{Chapter Conclusions}

This chapter presented a detailed account of the design and implementation of ANU-SOAM with its Data Service extension. The Data Service helps reduce communications and data transfers between client and SIs and differentiates it from other conventional SOA middlewares. The RM module allows scheduling and load balancing of tasks, which enables ANU-SOAM to respond effectively to heterogeneous environments. ANU-SOAM application development is supported by a set of APIs, which implements most of the Platform Symphony APIs and additional ones for Data Service functions. Client, resource manager and service libraries and a few configuration files help the compilation and deployment of applications in ANU-SOAM environment. ANU-SOAM could claim a significant place in the cloud stack and function as a PaaS for scientific application enabling real time applications on cloud. 


\section{Chapter 5}

\section{Programming Examples}

This chapter discusses different scientific kernels developed to evaluate the ANUSOAM middleware. Four major scientific kernels have been chosen to rigorously test the programmability, scalability and performance of ANU-SOAM under different scenarios. Each of these kernels have raised unique challenges and compelled ANU-SOAM to bring about new programming concepts and extend its APIs. Those four scientific kernels are:

1. N Body Solver

2. Conjugate Gradient Solver

3. Heat Transfer Equation Solver

4. Cholesky Factorization Solver (Left Looking)

\section{$5.1 \quad$ N Body Solver}

N Body is a classic problem in astrophysics and its counterpart - many body problem - is of vital importance in quantum mechanics[24]. A naive implementation of the N Body Solver (NBS) in astrophysics has $O\left(n^{2}\right)$ computation complexity [127]. A popular implementation of this algorithm is the simulation of planetary system with classic Newtonian laws of physics.

\subsubsection{Algorithm}

A naive NBS algorithm starts with a known set of values for the mass, velocity and position of bodies involved. The future positions and velocities of bodies can 
be predicted by iteratively moving forward in small - $\delta t$ - time increments. Basic algorithm of this naive NBS based on the derivation given in [128] is explained in Appendix C.6. This linear algorithm is parallelized and implemented using ANU-SOAM to evaluate its performance. The linear algorithm is split into client and service processes. Parallelization of algorithm involves assigning subset(s) of the $n$ bodies to each SI. SIs process these subsets as directed by each task from the client (refer to Algorithm 1).

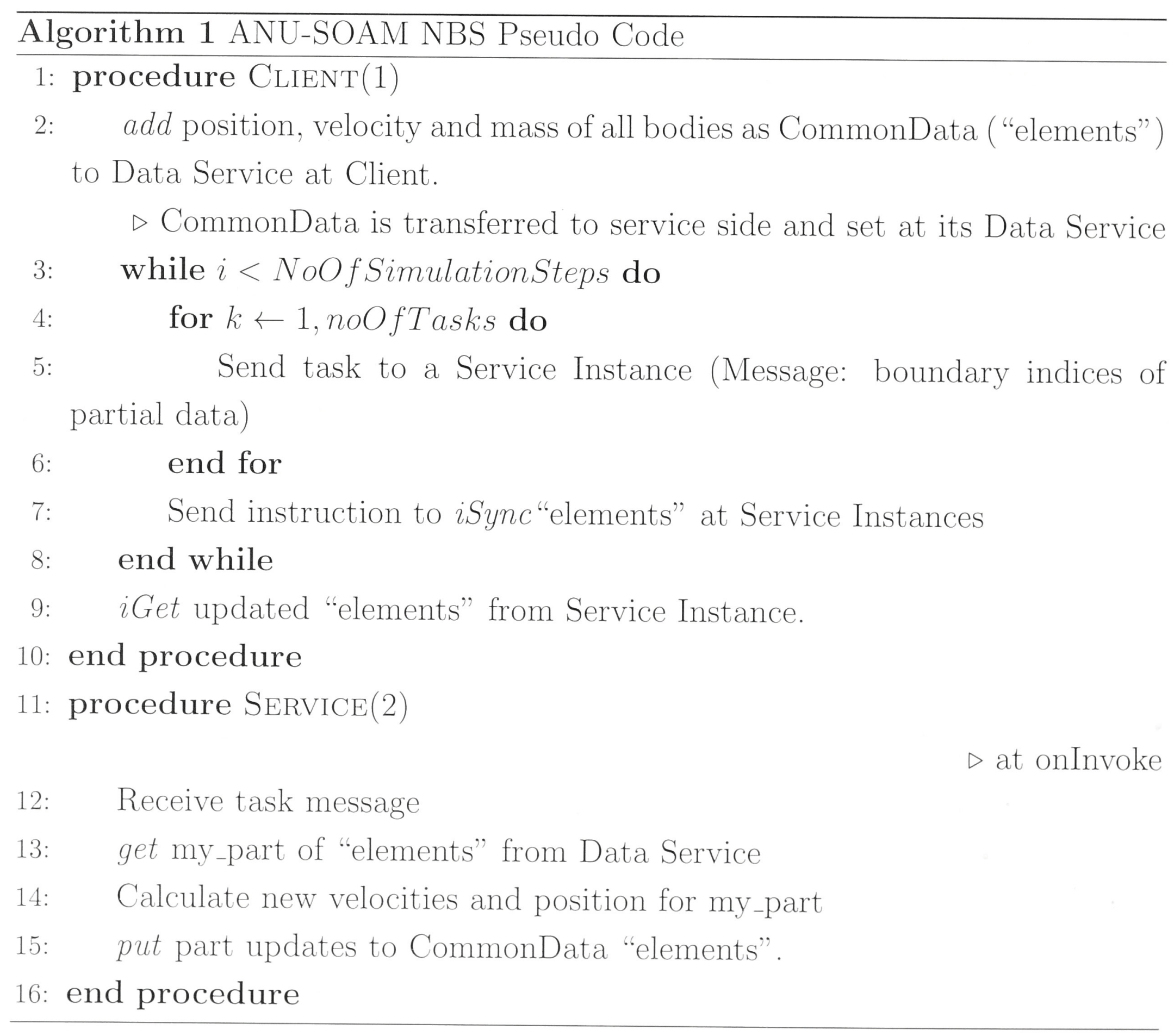

\subsubsection{Development Features}

\section{Generation of Tasks}

Since all bodies interact with each other in each time-step to produce updated values (new velocity and position) in this NBS algorithm, the algorithm will progress only in steps. These steps are called generations. 


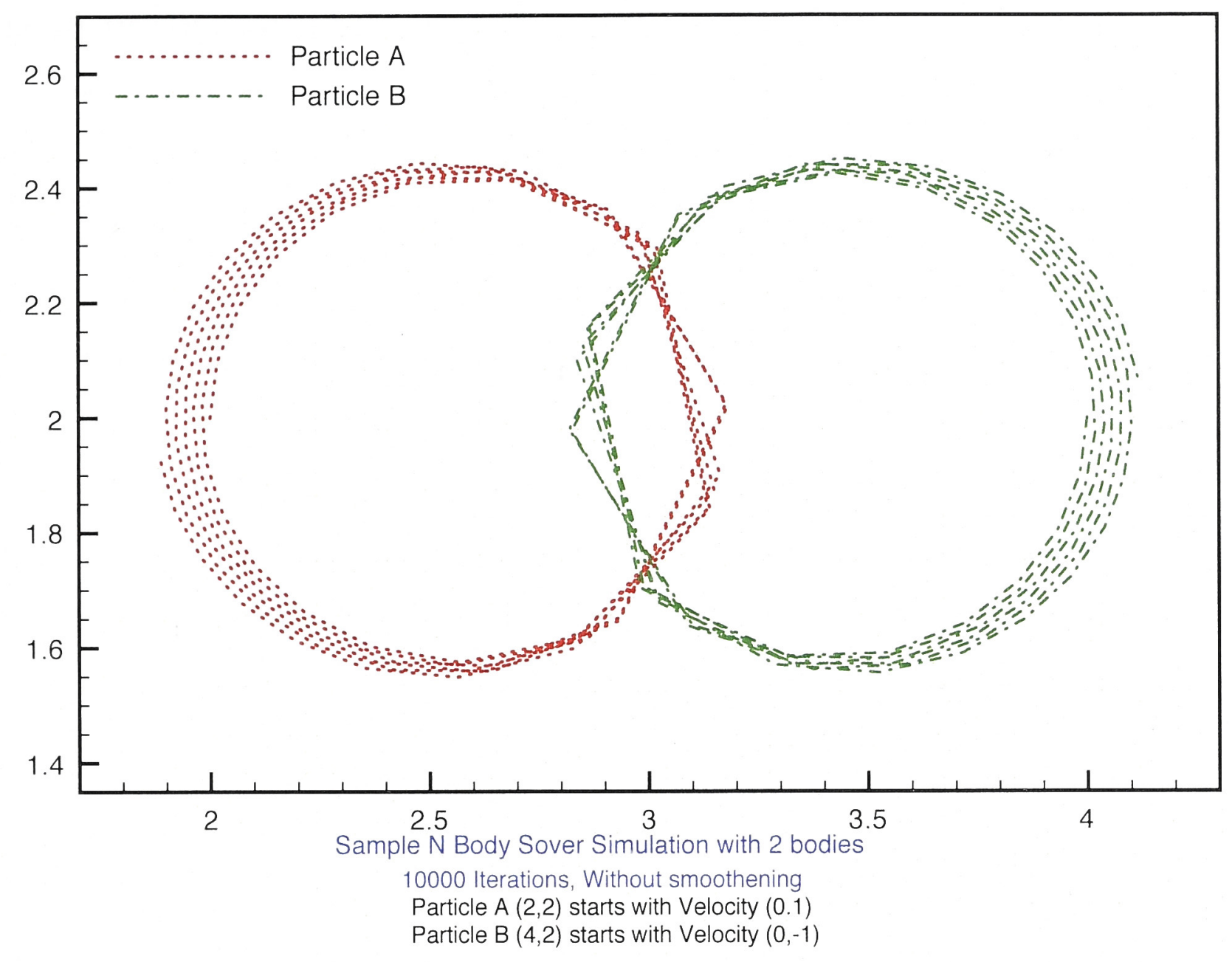

Figure 5.1: NBS Sample Simulation

\section{Use of Data Service}

The position, velocity and mass of a body are declared as a data structure. An array of these data structures are declared as a Common Data to be shared between client and service processes. This data remains the same for a generation of tasks in NBS. This property allows this Common Data to stay at SIs rather than communicating it back to the client after every generation. This allows the use of iSync rather than sync for most of the iterations in an implementation of NBS.

\subsubsection{Application Programming}

Client: The array of data structures consisting of the position, velocity and mass of all bodies is declared ( add) as Common Data. This will be available at the client and service processes. Task messages consist of boundaries of partial data to be processed at corresponding SIs. Once all task messages of a generation are despatched, iSync is called to synchronize the updated data at SIs. The process is repeated for the required number of iterations. For simulation (or any other) purposes, the updated values (position and velocity) can be accessed from the service side using the $i$ Get command, at any interval ( $i$ Get gets the data from any 
one of the SIs, but this process is kept hidden from the application programmer for convenience).

Service: An SI receives task messages by the onInvoke method and determines the boundaries of the partial job it has to execute. It then gets it partial data from the Data Service (which is declared as Common Data) and executes the partial parallel job. The updates are put into the Data Service (to the Common Data). It will be reflected only after the completion of all the tasks within a generation. This is achieved because Common Data put is a deferred action. The partial updates of tasks are synchronized among all SIs (but not transferred back to client) by an iSync command from the client. Whenever updated data is needed by the client, it uses an iGet command.

\section{Validation}

From Newton's law of energy we know that the total energy of a system shall remain the same for the system at any point of time. The algorithm is validated by calculating total energy at the beginning of the system and comparing it with same values at different iterations (a small margin of error is tolerated, because the naive NBS algorithm does not take care of smoothening effects etc). A sample simulation involving two bodies using the application is given in Figure 5.1.

\subsection{Heat Transfer Equation Solver}

When there exists a temperature gradient within a body, the Heat Transfer Equation describes the distribution of heat in a given region over time. The algorithm discussed in this section is a Heat Transfer equation Solver (HTS) in a twodimensional plane.

\subsubsection{Algorithm}

In HTS, the heat value (the temperature of an element) is dependent on the temperature of its neighbouring elements at any given time. The two dimensional heat matrix can be expressed as a cartesian grid. The change in temperature of each element of this grid (or matrix), with respect to the temperatures of its neighbouring elements is expressed in the algorithm given in Appendix C.8. This algorithm is derived from the sample codes explained in [129] and [130]. 


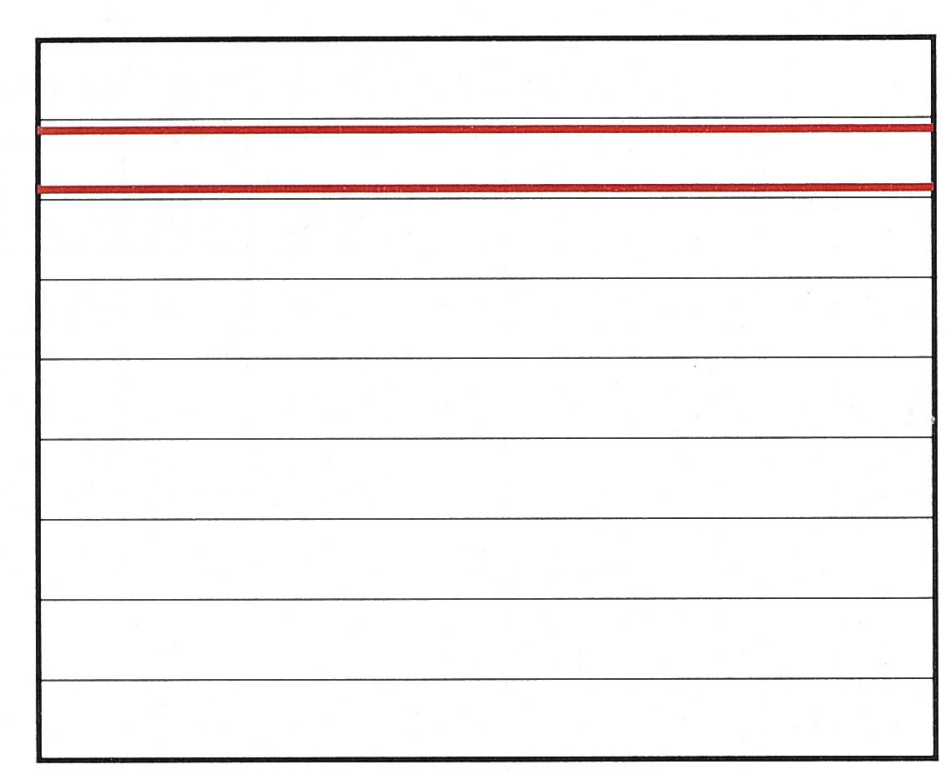

(a) as rows

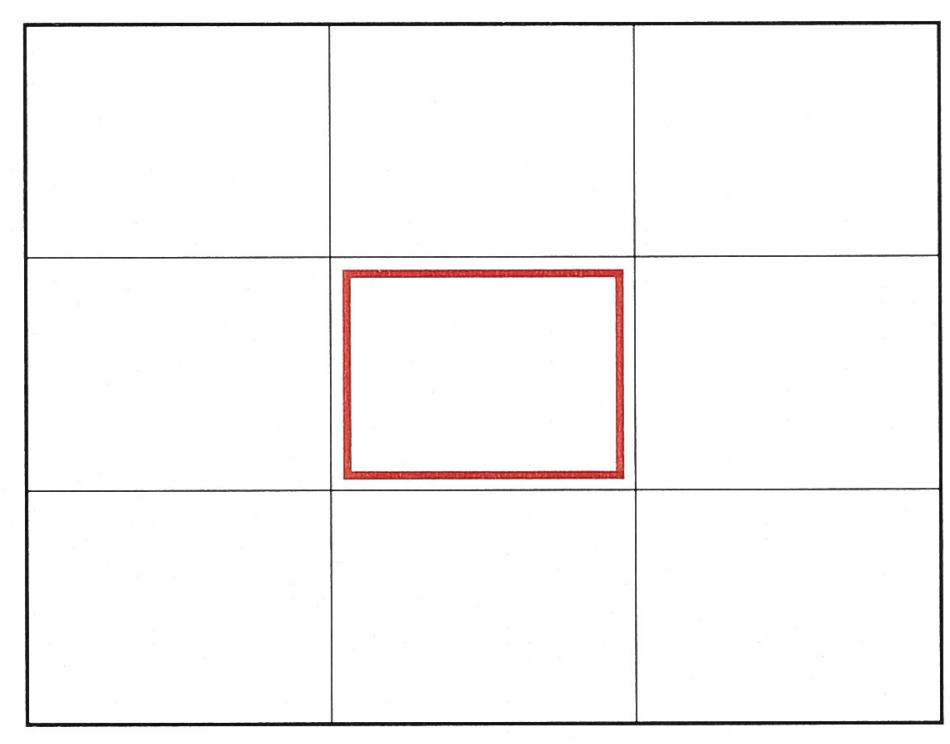

(b) as near-cube rectangles

Figure 5.2: HTS - Grid Breakup

The HTS algorithm raises a few challenges in parallelizing the algorithm using ANU-SOAM. Firstly, the matrix can be partitioned into sub-matrices for parallel processing. However, each sub-matrix needs to share only their borders with neighbouring sub-matrices in the HTS algorithm. This is because the inner elements of the sub-matrices (elements other than those on boundaries) need not interact with any of the elements in other sub-matrices. This property is unique to HTS, different to that of NBS.

Secondly, we had to make a choice to make on how the original data matrix is to be partitioned. It can either be partitioned along rows or as near-square rectangular blocks as given in Figure 5.2. As evident from the figures, partitioning as near-square rectangular blocks would give an advantage of the order of $4((\sqrt{(} p)-1) N / \sqrt{(} p)$ over partitioning over rows (where $p=$ perimeter and $N=$ the number of partitions) in terms of the amount of data to be synced using ANU-SOAM. As a result it was decided to divide the matrix into nearsquare rectangular sub-matrices for parallelization. The global indexing of the temperature matrix and the indexing for the partitions are given in Figure 5.3. ANU-SOAM pseudocode for HTS is given in Algorithm 2. 


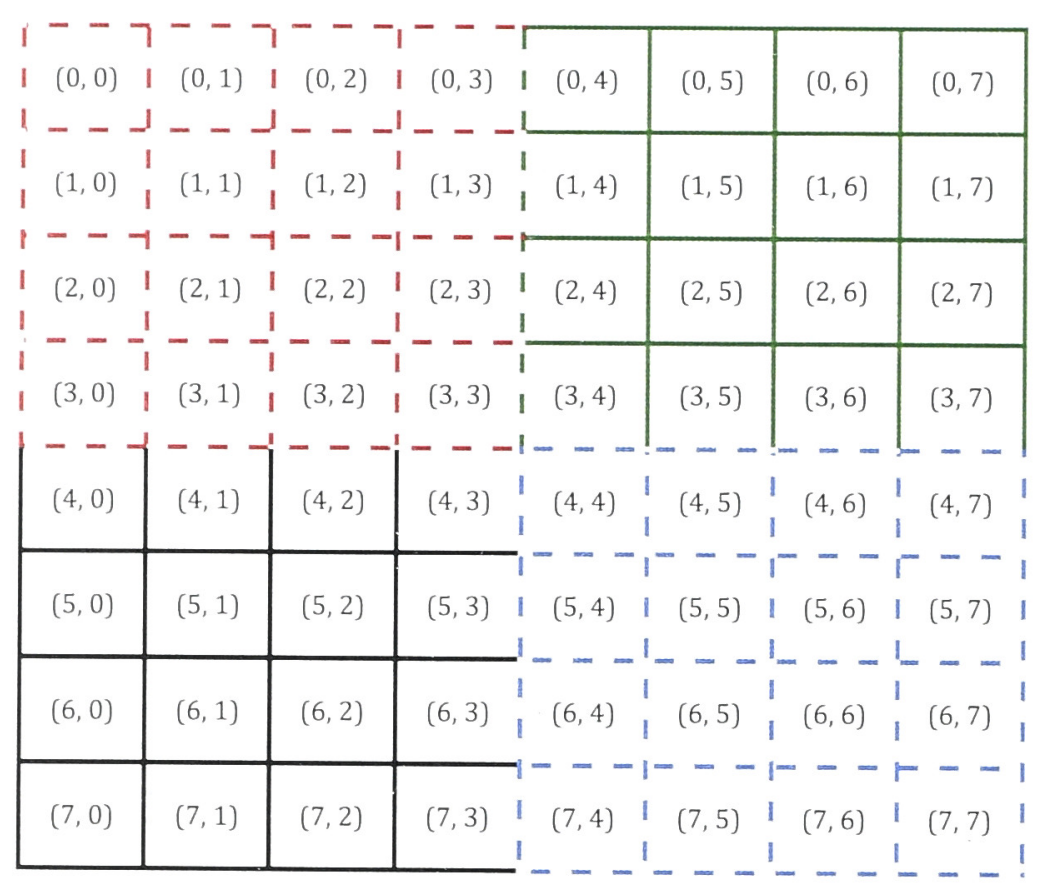

(a) Matrix Indexing

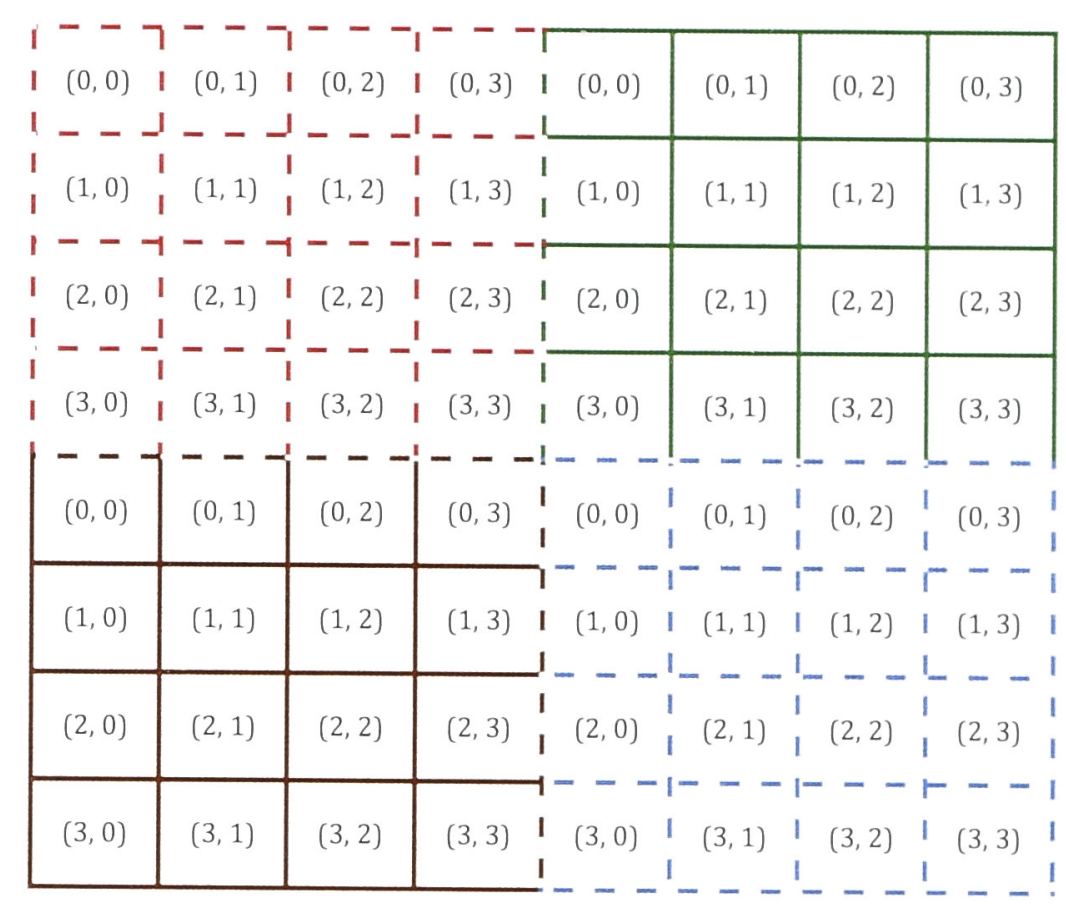

(b) Sub-matrix Indexing

Figure 5.3: HTS Data Grid Indexing - 1

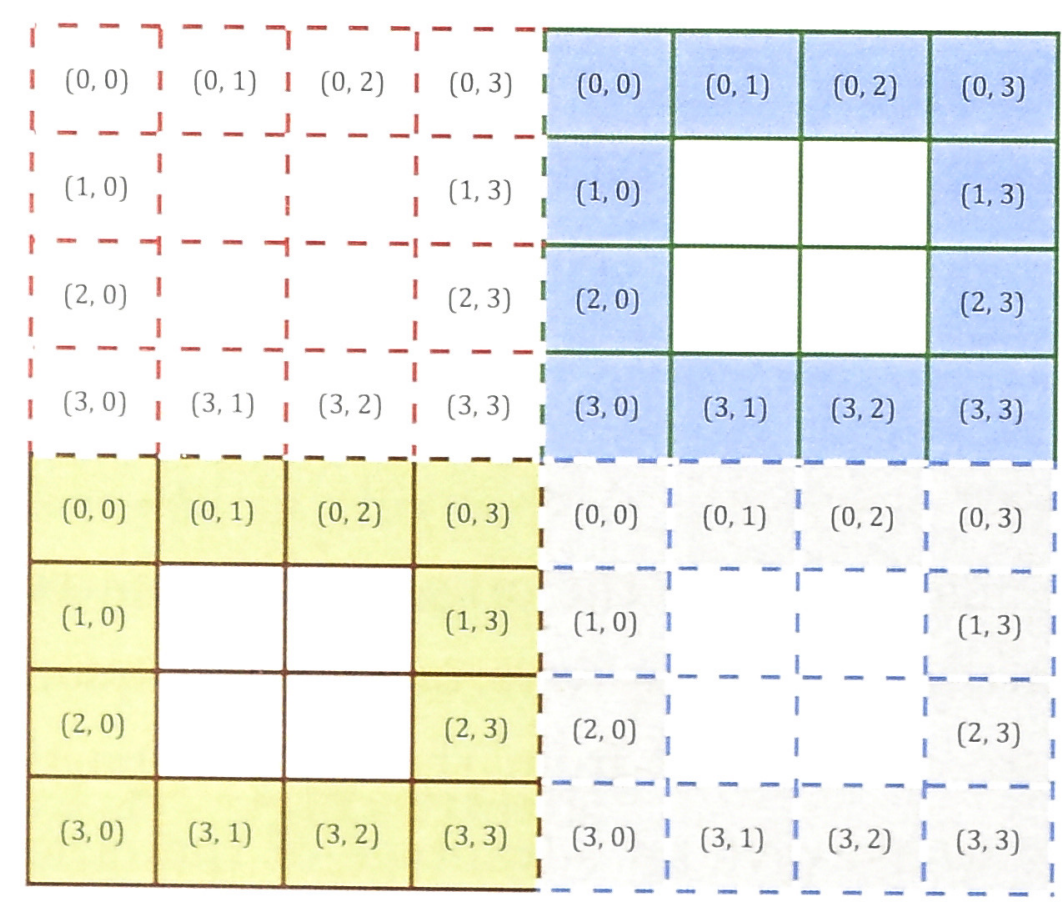

(a) Inner boundaries

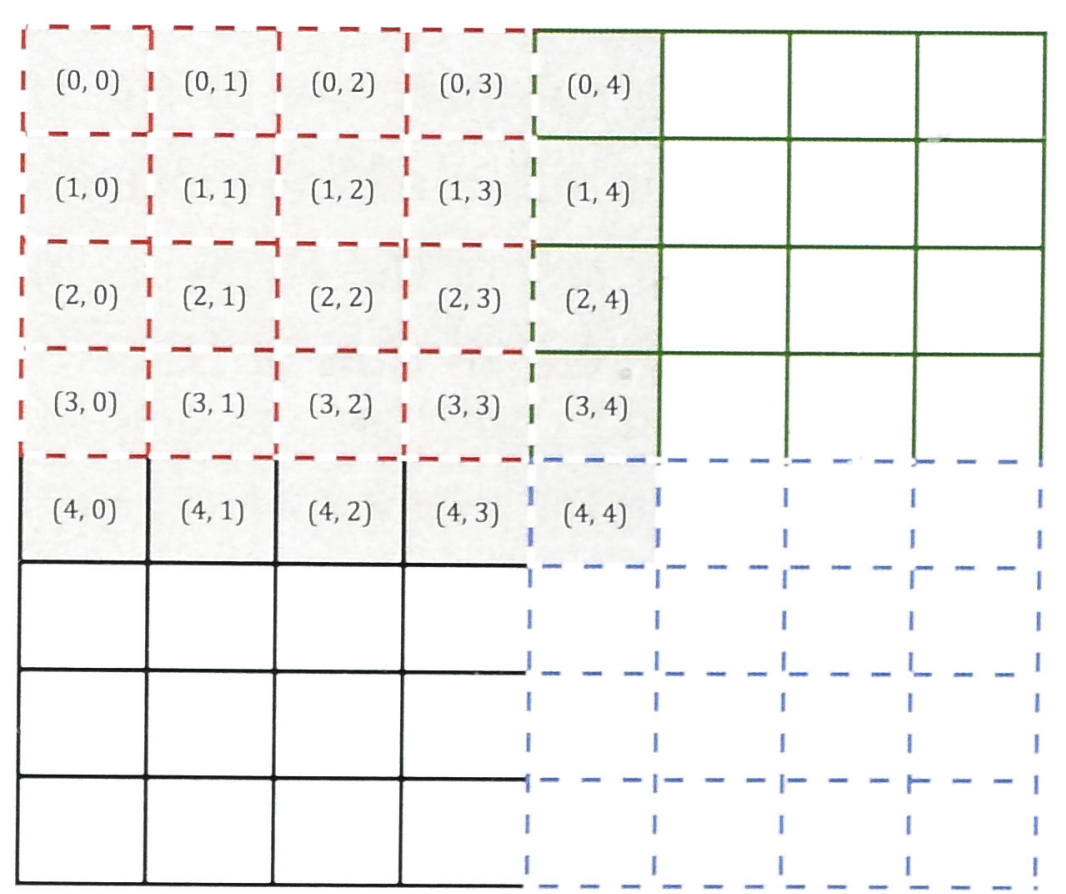

(b) Sub-matrix with ghost cells

Figure 5.4: HTS Data Grid Indexing - 2 


\subsubsection{Development Features}

\section{Use of $p u t_{-} b$ for Boundary Updates}

The special variant of put, put_b, is used to update the boundaries of sub-matrices. It will update only the four inner boundaries (boundary rows and columns) of the sub-matrix. These boundary elements are shown in Figure 5.4 (a). The put_b function will exclude the inner elements of the sub-matrix in the global sync. That is, the data communicated among SIs will be much smaller in this case. It makes the application more efficient and faster than if it uses the normal sync function. By synchronizing all inner boundaries, ghost cells for all sub-matrices are also updated.

\section{Use of $p u t_{-} i$ for Local Updates of Common Data}

Unlike NBS, all elements need not interact with all other elements in HTS. In other words, sub-matrix elements other than the boundaries can just be updated locally. put_i is used to locally update these elements within each SI.

\subsubsection{Application Programming}

Client: By adopting the algorithm in [129] the initial temperature is considered to be high in the middle of the grid and zero at the boundaries in ANUSOAM HTS implementation. The temperature at the boundaries are held at zero throughout the computation. The temperature matrix is declared (add) as a Common Data.

Boundary indices of parallelized sub-matrices and other parameters are set as part of the task message. These task messages are sent from client to SIs, and parallel computation is done and the results are updated (using put and put_b) in the Data Service. After each generation of tasks the boundaries of the sub-matrix are $i$ Synced. The updated temperature values are accessible at the client using iGet command.

Service: An SI receives a task message by the onInvoke method. From the task message it determines the boundaries of its sub-matrix and the partial computation is done by accessing its ghost cells as expressed in Figure 5.4 (b). The updated boundaries of sub-matrix (refer to Figure 5.4 (a)) are updated using $p u t \_b$ into the Data Service. The rest of the inner elements of the sub-matrix are 


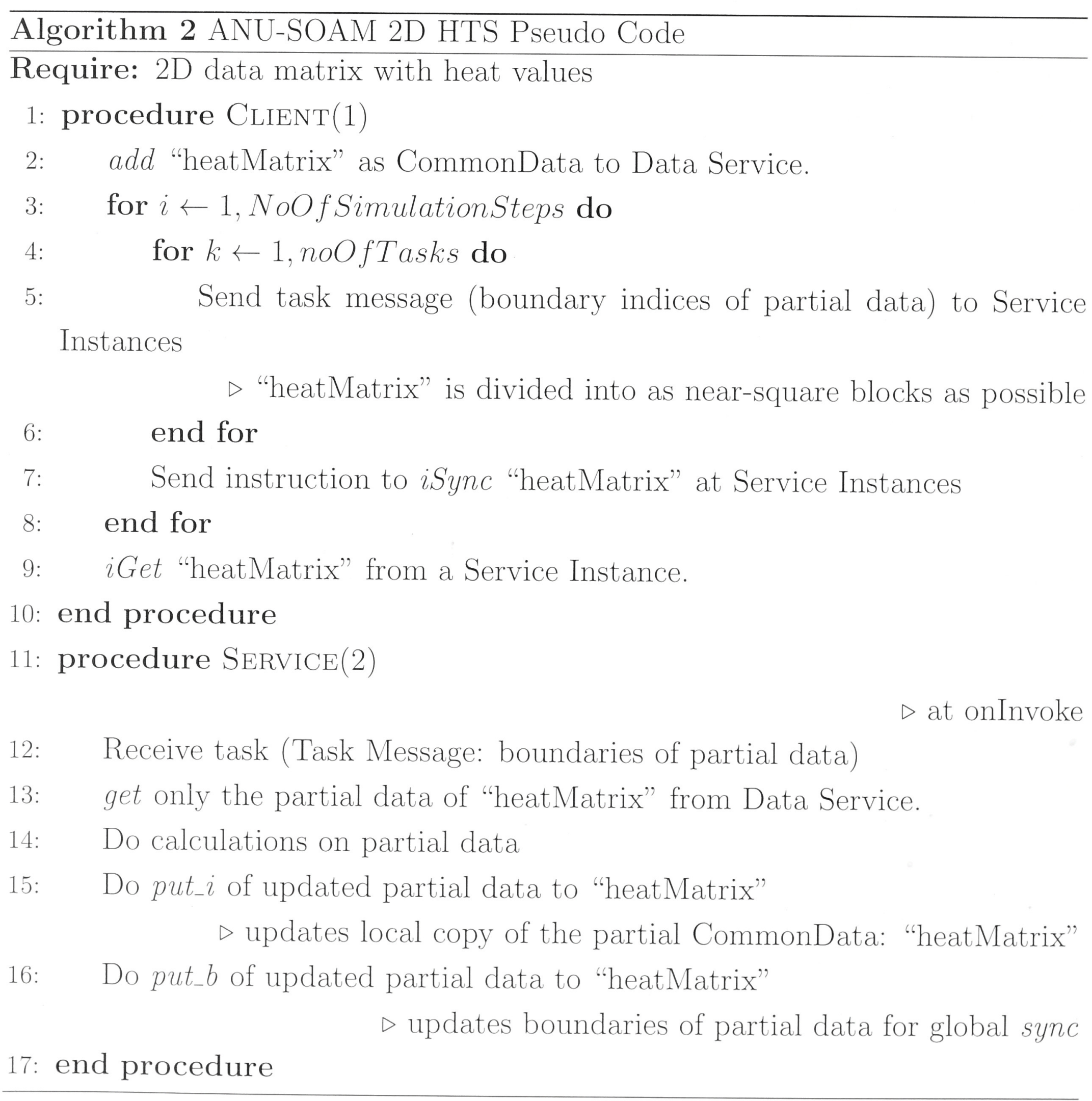


updated locally to Common Data using put_i. The boundary updates are globally synchronized to the Data Service after receiving the iSync command from the client, throughout all SIs.

\section{Validation}

As the heat transmission proceeds, the temperature along the matrix is supposed to get even. That is, the difference between the lowest and the highest temperature within the data matrix is expected to reduce consistently over successive iterations. The result matrix is checked to see that it is happening at regular intervals.

\subsection{Conjugate Gradient}

Conjugate gradient is a mathematical kernel used in many structural analysis problems in civil and mechanical engineering and to solve unconstrained optimization problems [28] [26]. Conjugate Gradient Solver (CGS) is considered to be a medium-grained but communication-intensive kernel.

\subsubsection{Algorithm}

The ANU-SOAM CGS algorithm is explained in my previous technical papers ( [89], [131]) and is reproduced here for the completion of this thesis.

The CGS is used to solve the linear equation: $Q * x-b=0$, where $Q$ is a given $N * N$ Positive Definitive Symmetric (PDS) matrix and $b$ is a given $N$ vector (Matrix $Q$ is PSD matrix when $Q=Q^{T}, x^{T} Q x>0$ for all $x !=0$ [132]). If the iteration number is $i, x_{i}$ is considered as the solution approximation, $d_{i}$ is termed as the search direction and $r_{i}$ is the residual. The technique is to perform iterations in the direction of $d_{i}$ till we find that the residual $r_{i}$ is small enough to be ignored to find the solution, $x_{i}$. Using the above logic, the iterations starts from an arbitrary initial guess of

$x_{0}=0$ and

$r_{0}=d_{0}=-Q * x_{0}+b$

Constants $\alpha$ and $\beta$ which are functions of $d_{i}, r_{i}$ and $Q$ determine the successive values of conjugacy constraints. This serial algorithm is further explained in PlanetMath.org [133] and presented in Appendix C.9.

This linear algorithm can be split into client and service processes in the 
ANU-SOAM implementation with varying degree of parallelization/complexity. This is because, the iterative process in CGS involves two main mathematical operations, namely,

1. Compute: $z=Q d_{i}$ (matrix-vector multiplication)

2. Reduce: $x_{i}, d_{i}$ (vector-vector operations) and Compute: $r_{i}$.

In the lowest level of parallelization, only the compute operation (matrixvector multiplication) is parallelized and all the reduction operations are done at the client side. This approach can be implemented using the conventional SOA or Platform Symphony approach, where the matrix is set as Common Data and the vector is sent as task messages to receive the partial Matrix-Vector multiplication task results at the client side. The pseudo code of this approach is given in Appendix C.10.

A higher level of parallelization can be achieved when both compute as well as reduction operations are parallelized. The ANU-SOAM client and service pseudo-codes of this highly parallelized CG algorithm are given in this section at Algorithm 3 and Algorithm 4.

\subsubsection{Development Features}

\section{Use of Non-replicated Two Dimensional Common Data}

Since the sub-matrices for partial computation of CGS are independent for computations from generations to generations, there is no need to fully replicate the matrix to all the SIs. This feature enables CGS to assign those sub-matrices as non-replicated two dimensional Common Data to respective SIs and linking them with corresponding tasks using a taskTag (refer to section 4.1.6).

\section{Use of onSessionEnter}

In CGS algorithm, the matrix (and thereby the non-replicated sub-matrices) remain the same throughout the iterations. This particular characteristic of CGS allows us to access the Common Data (sub-matrix) only once at the SI and to locally store it throughout the life of the process. The session specific method at service code, onSessionEnter allows us to implement it. This helps to reduce expensive Common Data accessing costs for successive iterations. 


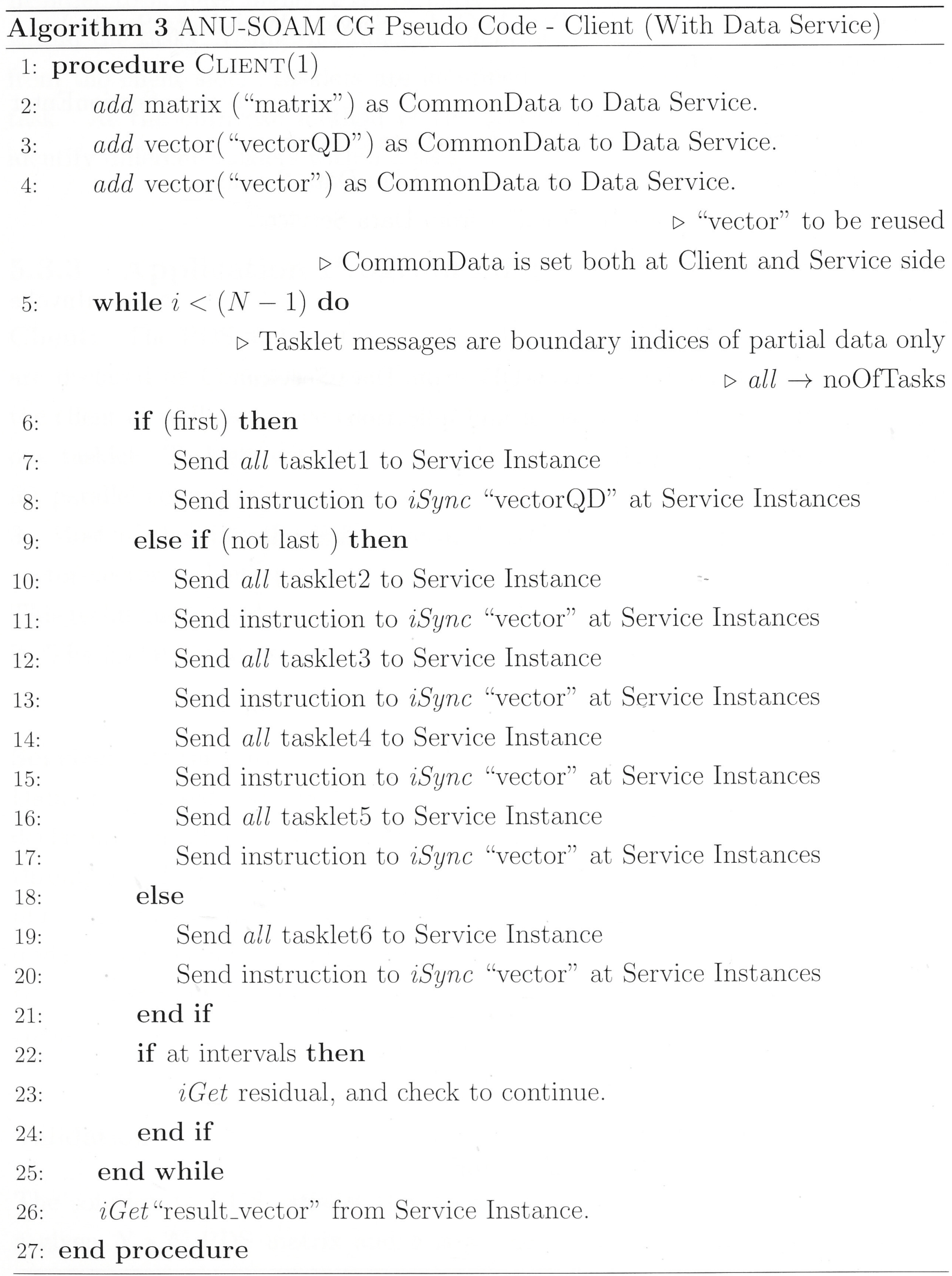




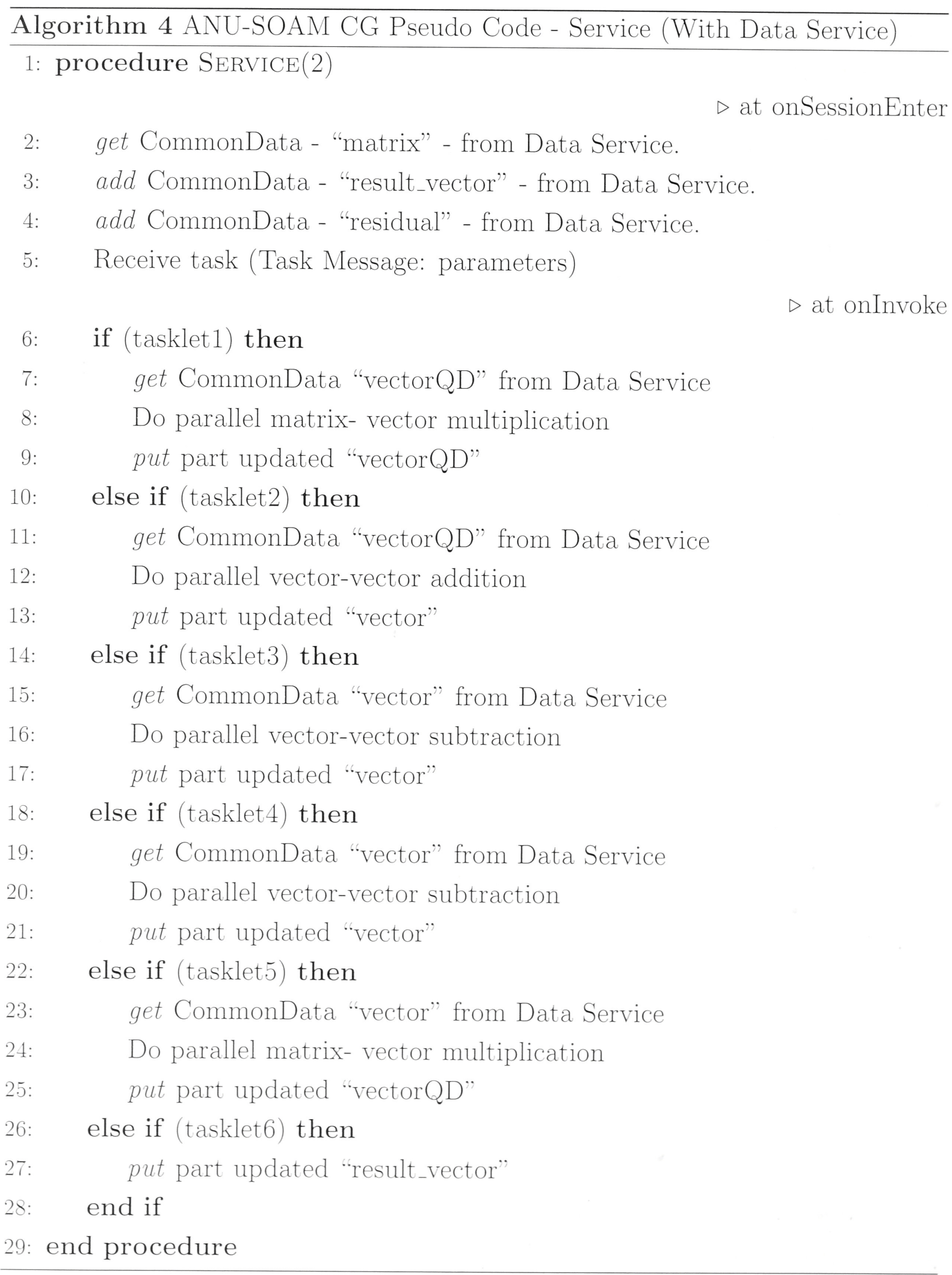




\section{Tasklets}

In order to achieve higher level of parallelization, the tasklets function is used within the tasks. Using this function, multiple tasklets can be called by a task from the client side. Tasklets are identified by a taskletTag associated with a task. At the onInvoke method of the service code a switch is introduced to identify different tasklets within a task.

\subsubsection{Application Programming}

Client: The PDS matrix, the initial vector and a buffer vector (to be reused) are declared as Common Data using add function. These will be available at the client as well as service processes. In this case, tasks can contain more than one tasklet. Tasklets with messages (boundary indices of sub-matrix) are sent for parallel computation at SIs as explained in pseudo code given in Algorithm 3. Most of the time the buffer vector is used to update the results of parallel vector-vector tasklet operations and iSynced after sending-each set of tasklets. This technique is used to reuse Common Data ("vector") rather than to duplicate it. The updated vector is accessible to the client using iGet command.

Service: At the initialization of an SI the sub-matrix (non-replicated Common Data) is accessed (get) and stored in the SI's local memory. This is done by declaring a memory holder in the method onSessionEnter, which is persistent throughout the life of a session. The SI receives the task at onInvoke and determines the tasklet from taskletTag and completes the partial computation. The updates are put into the Data Service to the relevant Common Data. The partial updates are synchronized throughout all SIs at the iSync command from the client.

\section{Validation}

The solution to CGS satisfies the linear equation: $Q * x-b=0$, where $Q$ is a given $N * N$ PDS matrix and $b$ is a given $\mathrm{N}$ vector. Find out the value of approximation vector $x_{i}$ and assign it to the left hand side above the equation. If the right hand side of the equation tends to zero, the solution approximation is considered acceptable. 


\subsection{Cholesky Factorization}

The Cholesky Factorization Solver (CFS) is a method for solving a linear system of equations. The CFS is commonly used to generate Monte Carlo simulations. CFS is used by mathematical applications that require safe tests for definiteness or to derive valid inequalities [134].

\subsubsection{Algorithm}

CFS factors a PDS matrix $A$ into the product of a lower triangular matrix and its transpose. It can be expressed as $A=L L^{T}$ [132]. There are right looking as well as left looking version of CFS. The left looking CFS is used to evaluate ANUSOAM as explained in [135] (in this thesis the term CFS refers to this left looking algorithm). The serial algorithm based on [135] is given in Appendix C.11. In this algorithm, there are two procedures - B2 and B1. Procedure-B2 is to update from columns to left to current column along row-wise (refer to Figure 5.5 (a)). Procedure-B1 is to scale the current column along column-wise (refer to Figure $5.5(\mathrm{~b}))$. Procedure-B1 includes two operations, the first one to find out $\sqrt{A[j][j]}$ and a second operation to calculate the rest of members in the same column under $A[j][j]$. Parallelizing left looking Cholesky algorithm in ANU-SOAM is achieved by doing computations on partial data (partitioned row-wise) for B2 and B1 at the service side. The pseudo code of this implementation is given in Algorithm 5 .

\subsubsection{Development Features}

\section{Changing Boundaries}

In all other kernels discussed in this chapter, the boundary indices of the sub-sets of Common Data to be processed using a particular task or tasklet (identified by a taskTag and taskletTag) remain the same for all the generations of tasks. But in CFS, as the computation progresses the boundary of the data under consideration for that particular generation changes. The Figure 5.5 (a) shows the boundaries of the matrix at iteration 3, for this particular example. This data is divided among 3 tasks as explained in the Figure using colour codes. But for the next generation, these boundaries will change for those 3 tasks. The whole matrix is declared as a Common Data and the boundaries of sub-matrices for each task is instructed from the client as task message to address this challenge. 


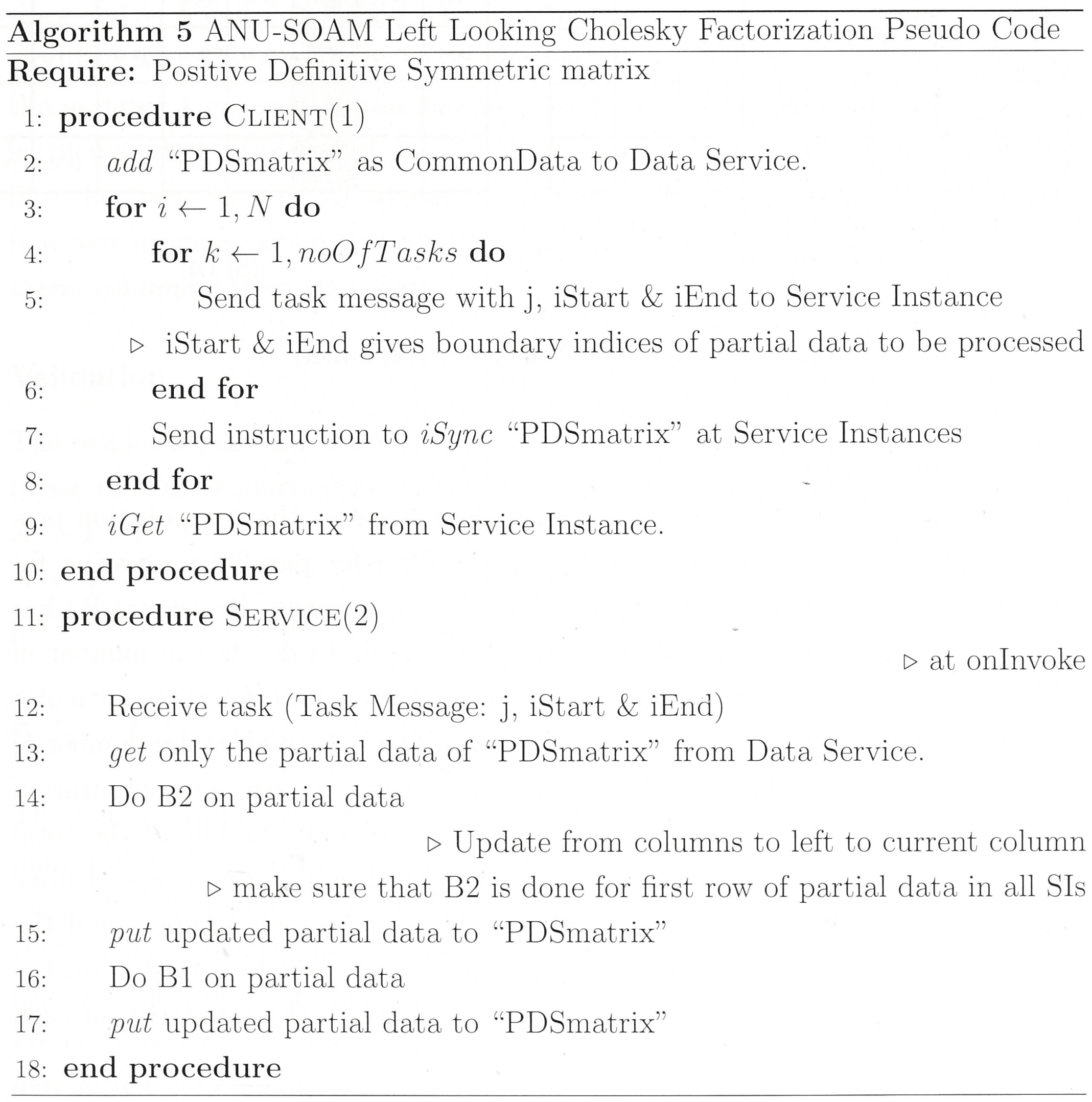




\begin{tabular}{|c|c|c|c|c|c|c|c|}
\hline$(0,0)$ & & & & & & & \\
\hline$(1,0)$ & $(1,1)$ & & & & & & \\
\hline $\begin{array}{l}(2,0) \\
-\end{array}$ & $(2,1)$ & $(2,2)$ & & & & & \\
\hline$(3,0)$ & $(3,1)$ & $(3,2)$ & $(3,3)$ & & & & \\
\hline$(4,0)$ & $(4,1)$ & $(4,2)$ & $(4,3)$ & $(4,4)$ & & & \\
\hline$(5,0)$ & $(5,1)$ & $(5,2)$ & $(5,3)$ & $(5,4)$ & $(5,5)$ & & \\
\hline$(6,0)$ & $(6,1)$ & $(6,2)$ & $(6,3)$ & $(6,4)$ & $(6,5)$ & $(6,6)$ & \\
\hline$(7,0)$ & $(7,1)$ & $(7,2)$ & $(7,3)$ & $(7,4)$ & $(7,5)$ & $(7,6)$ & $(7,7)$ \\
\hline
\end{tabular}

(a) $\mathrm{B} 2$

\begin{tabular}{|l|l|l|l|l|l|l|l|}
\hline$(0,0)$ & & & & & & & \\
\hline$(1,0)$ & $(1,1)$ & & & & & & \\
\hline$(2,0)$ & $(2,1)$ & $(2,2)$ & & & & & \\
\hline$(3,0)$ & $(3,1)$ & $(3,2)$ & $(3,3)$ & & & & \\
\hline$(4,0)$ & $(4,1)$ & $(4,2)$ & $(4,3)$ & $(4,4)$ & & & \\
\hline$(5,0)$ & $(5,1)$ & $(5,2)$ & $(5,3)$ & $(5,4)$ & $(5,5)$ & & \\
\hline$(6,0)$ & $(6,1)$ & $(6,2)$ & $(6,3)$ & $(6,4)$ & $(6,5)$ & $(6,6)$ & \\
\hline$(7,0)$ & $(7,1)$ & $(7,2)$ & $(7,3)$ & $(7,4)$ & $(7,5)$ & $(7,6)$ & $(7,7)$ \\
\hline
\end{tabular}

(b) B1

Figure 5.5: CFS Algorithm Explained

\section{Varying Task Numbers}

Since the size of data to be processed in CFS changes as the computation progresses, care must taken to equally divide the data for parallel processing for successive generations of tasks. It can be done at two levels. Procedure-B2 has the major chunk of workload in CFS. The first one is to divide the number of rows to be processed equally among the number of tasks used for that particular generation for the computation of B2. The second level is to alter the number of tasks during the iterations itself. It is noted that the data size for computation (thereby workload) for B2 tends be maximum towards the middle of the total number of iterations and least at the beginning and end of iterations. Unlike the three other application/kernels discussed before, the task numbers and the computational load (number of rows to be dealt with) shall be adjusted on the run for better performance by finding out the relative task granularity of each generation/iteration, in CFS.

\subsubsection{Application Programming}

Client: The PDS matrix is declared $(a d d)$ as Common Data. It is available at the Data Service at the client and service processes. Tasks with messages (boundary indices of parallelized data) are sent for parallel computation by Service Instances. After each generation of tasks the matrix is iSynced. The updated 
matrix is accessed from the service side using $i G e t$ command, at the last iteration.

Service: The Service Instance receives the task at the onInvoke method and determines the boundaries of the partial job to be done. It completes the partial computation B2 and results are updated to the matrix at the Data Service using put. Since this updated matrix is needed for the next compute operation B1, and since put is a deferred action, the local copy of the matrix at onInvoke is also modified with these updates. Now the partial computation of B1 is done using the updated local copy of the matrix. Care is taken to make sure that $\sqrt{A[j][j]}$ is calculated at all tasks before calculating the rest of B1. All B1 updates are put into the Data Service. All global updates of B2 and B1 to the Data Service are now synchronized among all SIs for next generation of tasks, after receiving the iSync command from the client.

\section{Validation}

The resultant matrix $L$ is assigned to the left hand side of the equation $A=L L^{T}$ to see that the equation satisfies the conditions. It can be- expressed as:

$A \rightarrow L * L^{T}$

$A^{\prime} \leftarrow L * L^{T}$

$\left\|A-A^{\prime}\right\| /\|A\| \Leftarrow 1.0 e-6$

\subsection{Chapter Conclusions}

The four scientific problems chosen to evaluate the programmability and performance of ANU-SOAM actually stretched the capabilities of ANU-SOAM, requiring its functionalities to be extended. Development of these scientific kernels - N Body Solver, Heat Transfer Equation, Conjugate Gradient and Cholesky Factorization - have brought about new functionalities like two dimensional addressing system (needed for HTS and CGS, refer to section 4.1.6), put_b function to update only the boundaries of a two dimensional data-set (needed for HTS, refer to section 5.2.2), and the dynamic task numbers (needed for CFS, refer to section 5.4). The pseudo codes of their ANU-SOAM implementations are discussed in this chapter and row algorithms appear in Appendix $\mathrm{C}$ for further reference. The validation techniques of these problems are also discussed in this chapter. 


\section{Chapter 6}

\section{Evaluation}

This chapter explains the experiments conducted to determine the scalability and performance of ANU-SOAM under various conditions and discusses their results. The experiments were conducted mainly under three test conditions:

1. Homogeneous Environment

2. Heterogeneous Environment

3. Cloud Environment

These experiments were conducted using four scientific kernels explained in the last chapter. In order to shed more light on the performance of ANU-SOAM, some of those kernels were compared against their MPI counterparts. Efforts were also taken to compare the performance of kernels using the ANU-SOAM programming model against that of the conventional SOA/Symphony programming model.

Each of these scenarios was executed 3 to 5 times and the average is used to produce the graphs in this chapter. In these experiments, the loading time or Common Data loading time is the time taken to set Common Data array(s) at the client and SIs within the Data Service. This is the initial setting time which has no influence on the rest of the computations and it is only a serious issue for computations of short durations with lower number of iterations/generations. The compute time consists of the time taken to process all generations of tasks by SIs and to receive and present the results at the client. 


\subsection{Homogeneous Environment}

\subsubsection{Homogeneous Experimental Environment}

The homogeneous experiments were conducted on the National Computational Infrastructure (NCI - Australia) Vayu cluster situated on the ANU campus, Canberra. Vayu is a Oracle/Sun constellation cluster which runs up to 1492 nodes on Sun X6275 blades. Each node consists of two quad-core $2.93 \mathrm{GHz}$ Intel Nehalem cpus with a 6.4GTs QPI bus. L1, L2 and L3 caches are 32KB (I) + 32KB (D), $256 \mathrm{~KB}$ and $8 \mathrm{MB}$ per quad-core cpu, respectively. It has a total of $37 \mathrm{~TB}$ of memory (RAM) on compute nodes, interconnected using an on-board QDR InfiniBand adapter. The nodes run the CentOS 5.6 Linux distribution (based on RHEL 5.6) and the batch queuing system is supported by OpenPBS [136].

ANU-SOAM middleware libraries and all kernel executables used for these experiments were compiled with level 3 optimization (-O3) and deployed on the Vayu cluster as explained in section 4.3. Each experiment was submitted using the OpenPBS queuing system available on Vayu.

\subsubsection{Performance and Speedup}

The aim of these sets of experiments was to analyze the performance and scaling capabilities of ANU-SOAM infrastructure using the scientific kernels under consideration. Each experiment reserved up to 32 cpus and 40 GB of memory using a job description on OpenPBS. The round-robin scheduling policy - which considers all nodes are equal in performance - was adopted for all these experiments because a homogeneous environment is assumed within the Vayu cluster. The OpenPBS queuing system in Vayu deploys one process (SI) each on each node. Generally, the number of tasks were equal to the number of SIs in these experiments to ensure proper load balancing under homogeneous conditions. The results were collected and compiled into performance and speedup graphs. The curves in these graphs are based only on compute time, for the loading time is always a constant for any number of iterations. The scaling or speedup is defined as $T(1) / T(n)$ where $T(1)$ is the compute time to execute the particular code using single SI and $T(n)$ is the compute time to execute the same code using $n$ SIs. To study the effects of the number of tasks, experiments were conducted with varying task numbers and reported separately. 


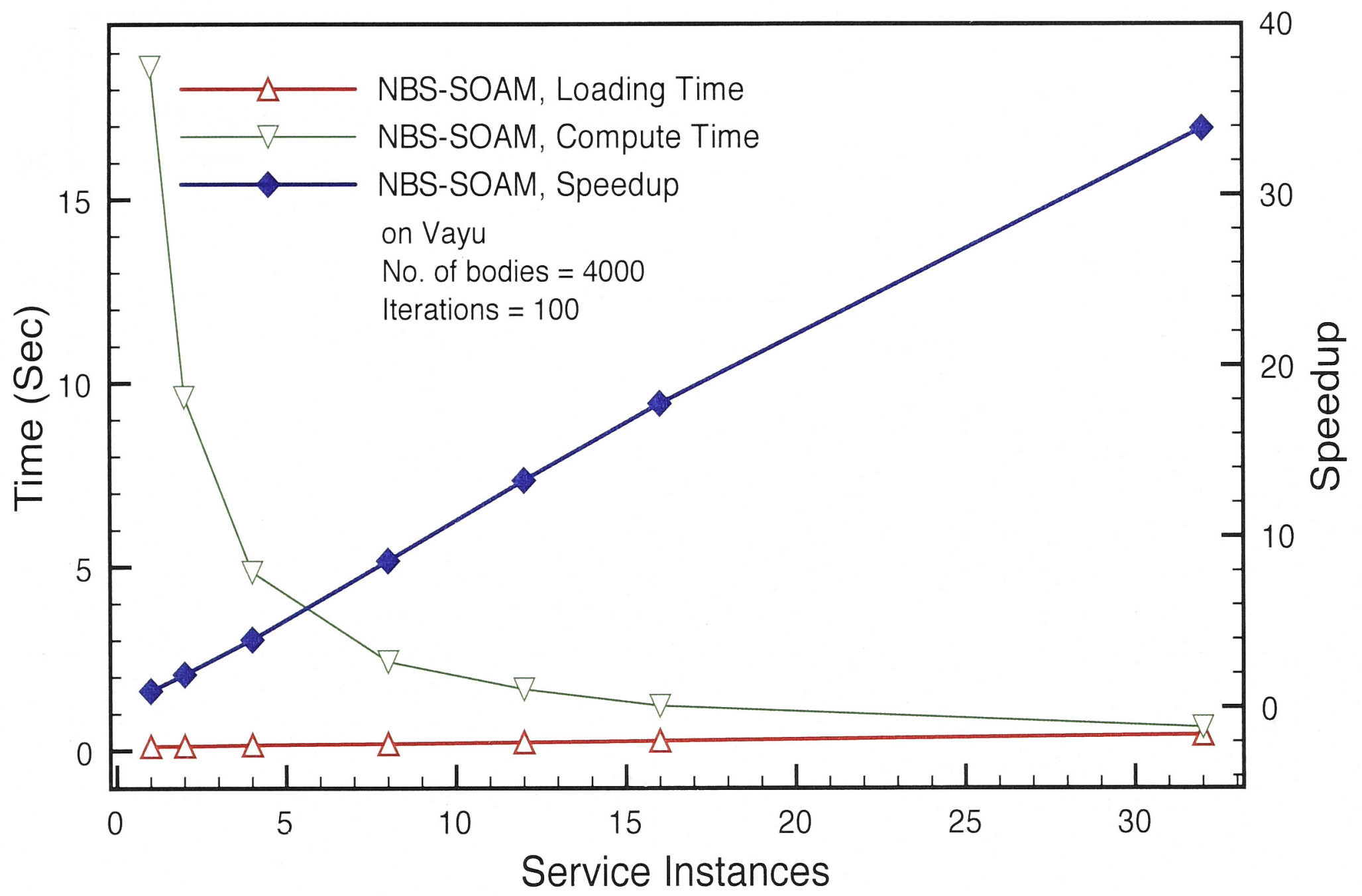

Figure 6.1: NBS: Performance and Speedup

\section{N Body Problem}

The ANU-SOAM NBS kernel explained in section 5.1 was submitted to the NCI Vayu cluster. A computation of positions and velocities for 100 iterations for 4000 bodies was carried out in all experiments. Figure 6.1 shows an acceptable level of performance improvement for up to 32 SIs on 32 Vayu nodes. ANUSOAM scales well in almost a geometric fashion. It was noticed that the naive NBS algorithm used in this implementation has a task granularity of average 0.22 seconds which turns out to be the optimum task granularity for ANU-SOAM to produce ideal scaling of its applications. The results show that algorithms with optimum computation to communication ratio (or optimum task granularity) can be effectively processed with ANU-SOAM.

Loading Time: The results in Figure 6.1 also shows that the ANU-SOAM NBS has significant initiation time or Common Data loading time, especially as the number of SIs increase. This increase is expected because the initial data (Common Data) has to be transferred to more compute nodes as number of SIs increase and there is a cost involved in doing so. However this overhead 


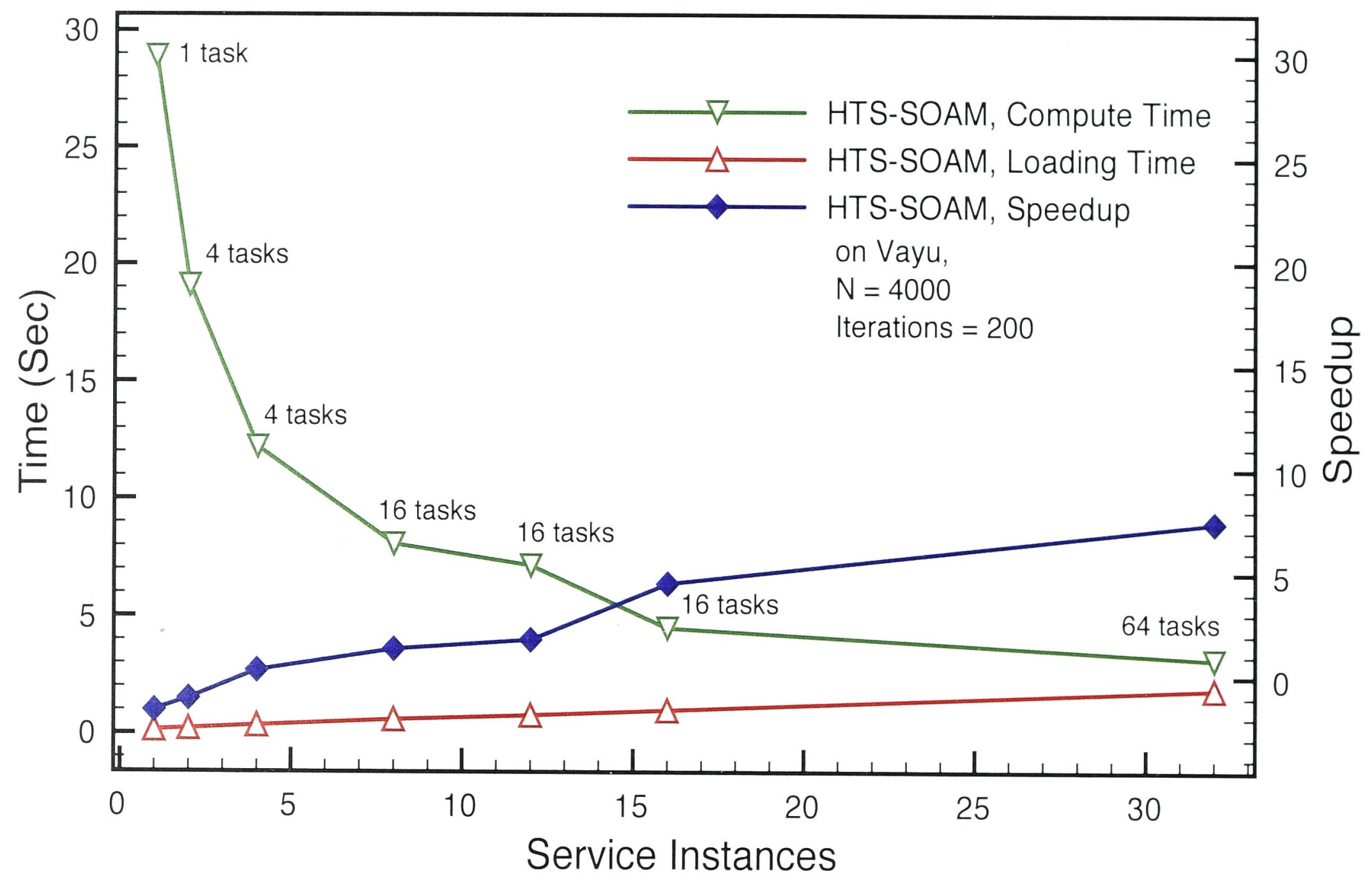

Figure 6.2: HTS: Performance and Speedup

is relatively lower for NBS than than that of other kernels like CGS (refer to Figure: 6.3). It is because the Common Data is set as a single dimensional array in the Data Service (API call is: addCommonData(name, PtrToDataTypeArray, numberOfElements)) and there is only one Common Data array declared in the Data Service in this case. 100 iterations are used only in experimental conditions. In real life there will be significantly higher number of iterations and the loading time will have very less effect on the total compute time.

\section{Heat Transfer Equation}

The ANU-SOAM HTS kernel explained in section 5.2 was submitted to the NCI Vayu cluster. The heat transmission within a two dimensional plane of order 4000 is computed for 200 iterations for all experiments. The performance as shown in Figure 6.2 shows an acceptable level of speedup to be considered to be a high performance outcome. However, the scaling is not as good as NBS because the computation to communication ratio is not optimum for ANU-SOAM in the HTS algorithm. For programming convenience, the two dimensional data grid is partitioned with an equal number of rows and columns (as explained in Figure 
5.2). This means that the number of partitions and therefore the number of tasks will be the square of 1, 2, 4, 6, 8 etc. The apparent performance/scaling anomaly using 12 SIs in the graph is due to this limitation of the algorithm, where we have 16 tasks to be divided among 12 SIs, which creates a load imbalance.

Loading Time: The loading time is higher in HTS compared to NBS. The higher loading time can be attributed to the fact that Common Data in HTS is expressed as a two dimensional (2D) array (matrix) (API call is addCommonData(name, PtrPtrToDataTypeArray, int ndim, int* dims)), unlike the one dimensional Common Data array in NBS. There are computational costs involved in using a two dimensional array to define Common Data within ANU-SOAM as explained in section 4.1.6. Expressing a matrix as a two dimensional array is convenient from the programming perspective, but this result shows that there will be a trade-off in terms of higher loading time between convenience and performance in using two dimensional Common Data.

\section{Conjugate Gradient}

Two versions of the CGS kernel were developed for experimental purposes. Version 1 uses the Data Service but with smaller number of tasklets. That is, tasklets 2 to 5 described in the CGS algorithm (refer to section 5.3.1) are grouped into a single tasklet (the vector-vector operations are not parallelized in this case). The idea is to increase the average task granularity of each tasklet and to reduce the total number of tasklets. In Version 2, the whole CGS algorithm is parallelized to the maximum possible way having a total of 6 tasklets in its implementation. This is achieved by not only parallelizing the matrix-vector operations of CGS but alṣo all the vector-vector operations as well. The first and the last tasklet in both the versions are used only at the first and last iterations respectively for the logical consistency of the algorithm. So the analysis of tasklet loads can be based only on the middle tasklet(s), ignoring the time taken to process first and last tasklets in both the versions.

A matrix size of 4000 was used to test the performance of both versions for up to 32 nodes on the Vayu cluster. A constant number of iterations - 441 was used for all the experiments to ensure consistency in results (it was noted that 441 iterations produced enough fineness for the CGS residual for most of the experimental scenarios). The results show that (refer to Figure 6.3) Version 1 produces better results than Version 2, which is expected. It is because Version 


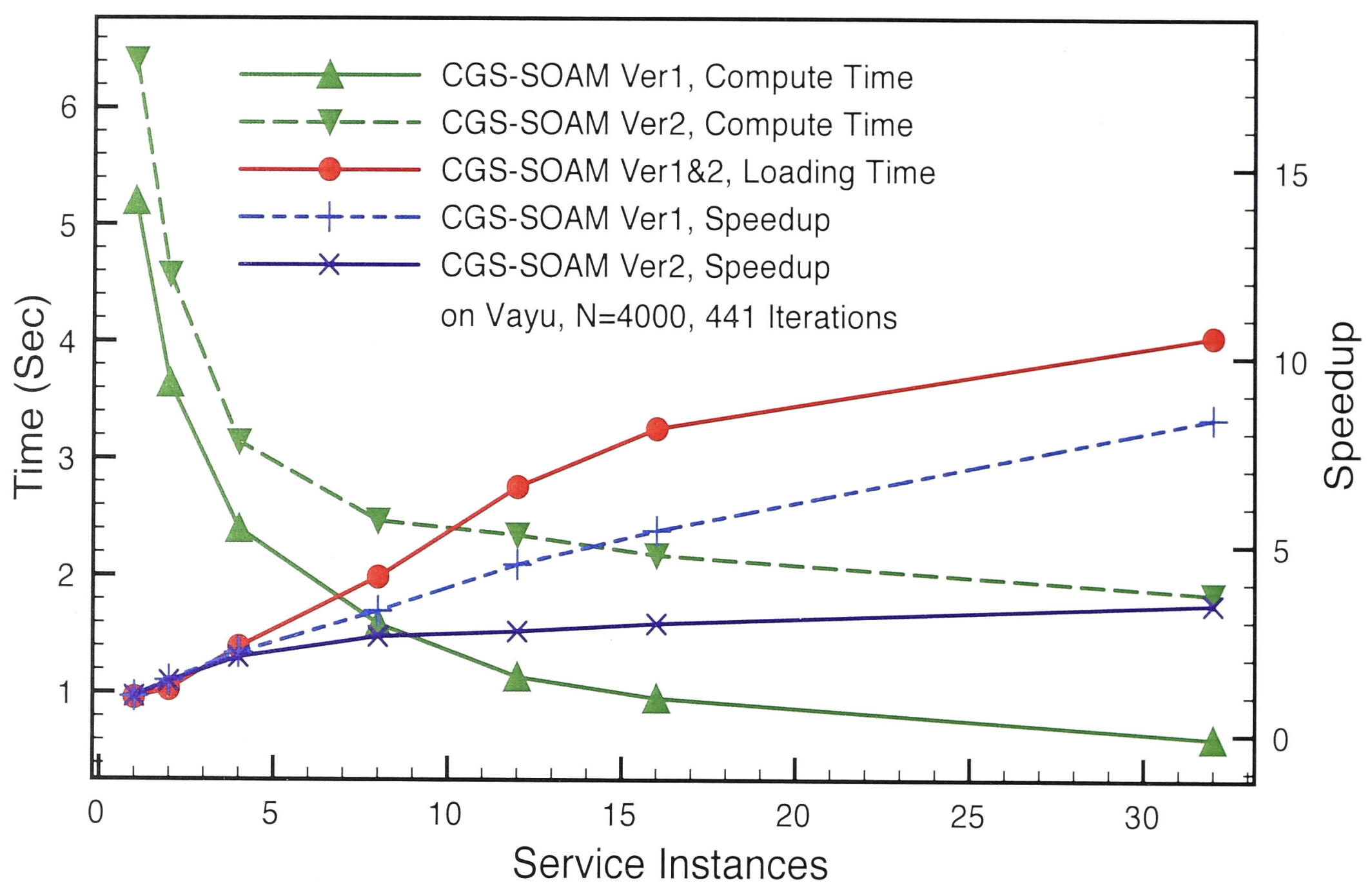

Figure 6.3: CGS - Version 1\&2: Performance and Speedup

1 cuts down the communication overheads with a lower number of tasklets and thereby increasing the average tasklet load (refer to Table 6.1). The scaling results are not as good as the NBS for the computation to communication ratio is not quite optimum for ANU-SOAM in parallelizing CGS algorithm. Still the experiment results can be considered acceptable in producing near enough high performance outcomes, within the limitations of an SOA model.

Effect of using more Tasklets: Version 2 uses more tasklets - 6 in number - than Version 1 which uses only 3 tasklets. An analysis of the tasklet compute time (tasklet granularity) at SIs (by putting time stamps in all the tasklets at the service code) of Version 2 shows that granularity of tasklets 2 to 4 (parallelized vector-vector operations) are negligibly smaller compared to that of tasklet 5 (parallelized matrix-vector operation) (refer to Table 6.1). There is always a cost involved in creating more tasklets. Moreover, there are additional overheads involved in synchronizing the data after each set of tasklets in the Version 2 algorithm (refer to section 5.3.1). As we increase the number of SIs, these overheads accumulates and are responsible for the poor scaling performance of CGS Version 
2 compared to that of Version 1.

The CGS Version 2, however, demonstrates that we can develop sophisticated applications by using tasklets function in ANU-SOAM. If all the tasklets have optimum workloads in an application, we could assume that the computational advantages in generating those tasklets will surpass the communication and administrative overheads in generating them and improve the total performance of the application, which may not have been achieved without the tasklet functionality.

Table 6.1: CGS-SOAM Tasklet Time*

\begin{tabular}{|c|c|c|c|c|}
\hline $\begin{array}{c}\text { Version 1 } \\
\text { Ave. (Sec) }\end{array}$ & \multicolumn{4}{|c|}{ Version 2} \\
tasklets 2-5 & tasklet 2 & tasklet 3 & tasklet 4 \\
& v-v addition & v-v subtraction & v-v subtraction & tasklet 5 \\
m-v multi \\
\hline 0.00431 & $4.38 \mathrm{e}-05$ & $4.29 \mathrm{e}-05$ & $4.28 \mathrm{e}-05$ & 0.00404 \\
\hline \multicolumn{5}{|c|}{ *on Vayu cluster using 8 SIs \& N=4000 } \\
\hline
\end{tabular}

Loading Time: In both CGS implementations, two Common Data arrays are used. One is used for the original matrix, which remains the same throughout the operations, and the other is a holder for the vector which is updated and synchronized after each generation of tasks/tasklets. The higher loading time of both CGS versions are due to this increased number of (and corresponding volume) Common Data to be communicated to all SIs compared to NBS implementation. This result shows the importance of using Common Data in moderation in developing applications for achieving overall better performance of ANU-SOAM applications or kernels.

\section{Cholesky Factorization}

The CFS kernel was developed using ANU-SOAM APIs as explained in section 5.4 and was submitted to the NCI Vayu cluster. The input matrix was expressed as a two dimensional array (API call is addCommonData(name, PtrPtrToDataTypeArray, int ndim, int* dims)) and in the first version (Version 1), full access of the updated matrix from the Data Service was envisaged for all tasks. A matrix order of 4000 used for experiments which meant that it takes 4000 iterations to produce the final result (because in CFS, iterations $=N$ ). Even though this approach seems to scale well, it did not produce the high performance outcomes we 


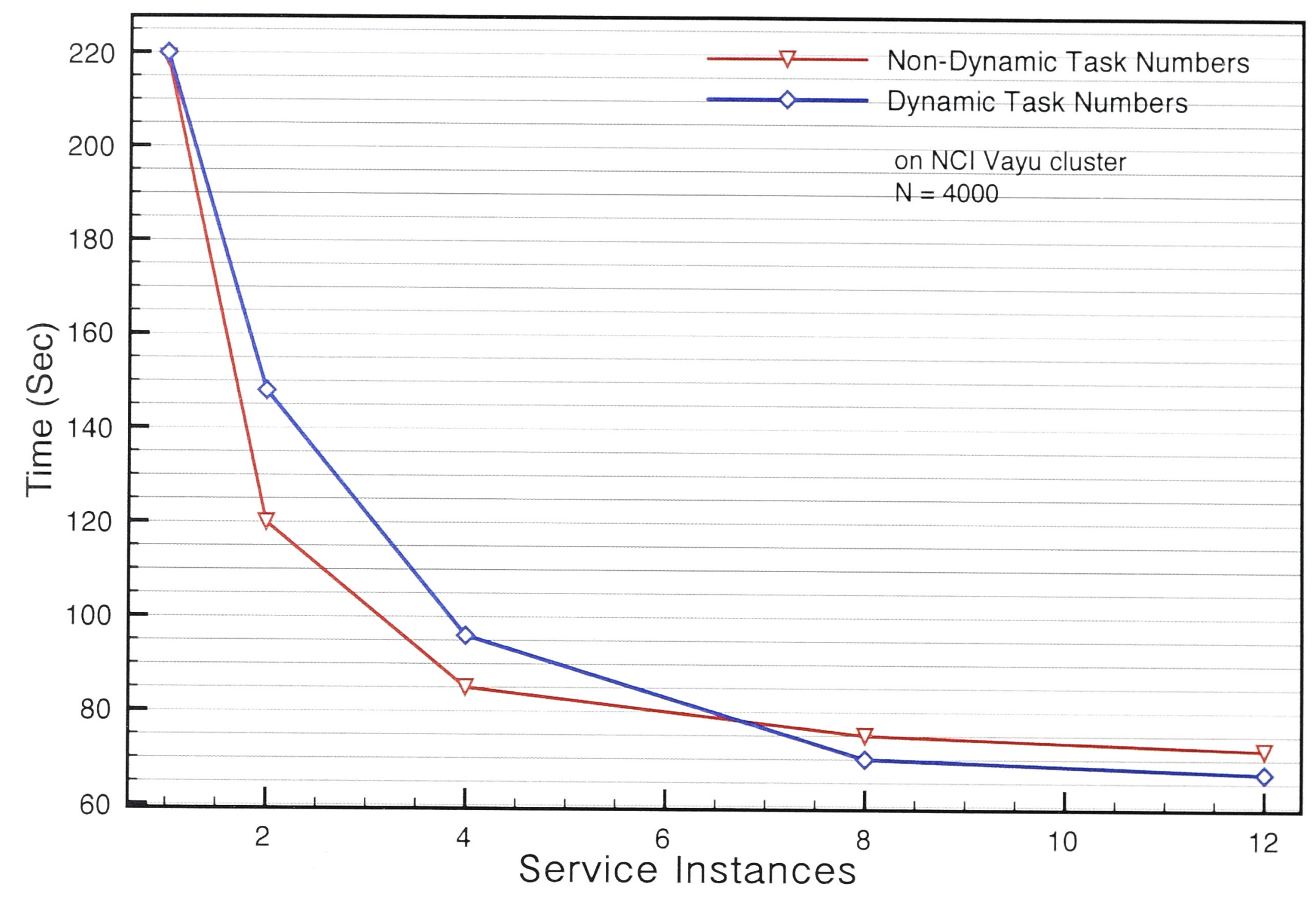

Figure 6.4: CFS (With Full Matrix Access): Performance

expected (refer to Figure 6.4). The following approaches were adopted to address this performance issue.

Dynamic Task Numbers: The nature of the CFS algorithm suggests that the compute load will be higher towards the middle of the iterations. The Version 1 approach was tweaked slightly to send varying number of tasks as we progress through the iterations. The task numbers for a particular iteration were calculated at run time based on its relative compute load to that of the maximum expected load, where the maximum task numbers were assigned at the middle of iterations and a minimum of one task assigned at the beginning and at the end of the iterations. The RM was modified accordingly to properly direct those varying tasks to SIs, for load balancing policies random and round-robin. This approach slightly changed the scaling behaviour of the kernel, but not without any considerable performance improvements (refer to Figure 6.4).

Improving Common Data Access Time: Later, ANU-SOAM CFS algorithm was improved by getting only the partial data (sub-matrix) by a task just 


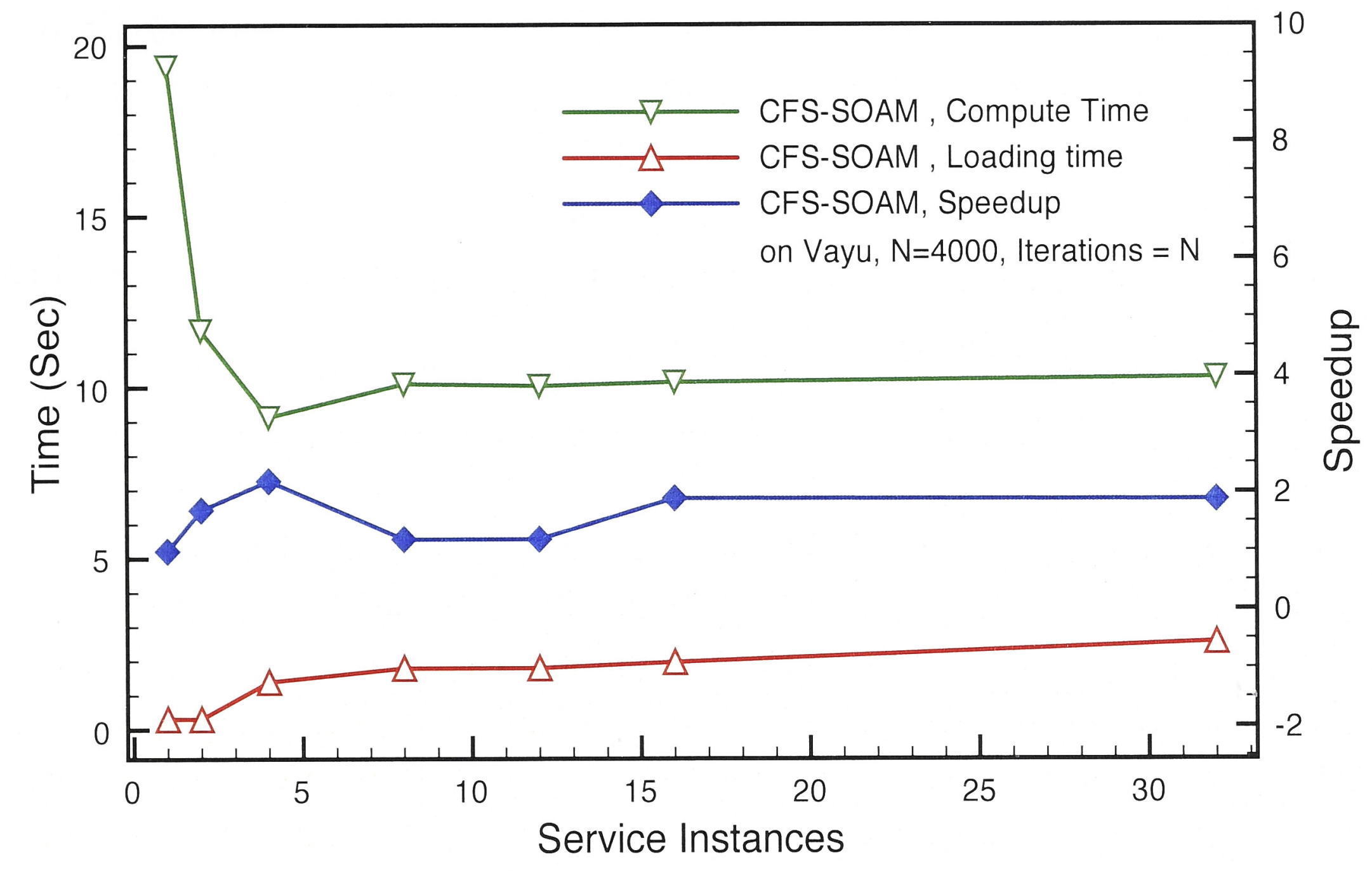

Figure 6.5: CFS (With Partial Matrix Access): Performance and Speedup

to do its part of the partial computation from the Data Service (CFS Version 2). The programming complexity increased slightly, but this dramatically cut down the Common Data accessing time and considerabley improved the performance for a similar set of matrix using Version 1 (refer to Figure 6.5). However, Version 2 failed to scale beyond 4 SIs. This can be attributed to the lower (and not optimal) task granularity of CFS parallelization. Our experiments showed that the average task granularity of CFS is 0.0006 seconds as compared to 0.22 seconds of NBS. That tends to suggest that CFS is a fine-grained parallel kernel rather than a medium-grained one for ANU-SOAM. The granularity of tasks is also a function of the data size each task is assigned to process. But in this case we were not able to increase it because it will hit the memory limit of the compute nodes that support the Data Service, which relies on its memory.

\section{Inference}

The results generally indicate that ANU-SOAM can produce high performance outcomes for medium-grained scientific problems, even though the scaling behaviour may vary depending upon the algorithms and ANU-SOAM functionali- 
ties used. These high performance outcomes were not possible using conventional SOA middleware like Platform Symphony which did not have a Data Service and relied on tools other than MPI for communication infrastructure (refer to sections $2.4 .2,4.1 .1$ and [89]).

\subsubsection{Performance Comparison Against Conventional SOA Programming Model}

In a conventional SOA programming model, there is no data service. It means SIs cannot manipulate Common Data at compute nodes. It may result in increased data communications between the client and SIs, which could have been avoided using a data service. Experiments were conducted to investigate this assumption.

The Platform Symphony model is a conventional SOA programming model which has no data service extension. ANU-SOAM implements the architecture and APIs of Platform Symphony which makes it possible to develop applications in ANU-SOAM that resemble conventional the SOA/Platform Symphony programming model. As a result, conventional SOA/Symphony style NBS and CGS were developed using ANU-SOAM (but without using the Data Service functionalities) and compared them against similar ANU-SOAM applications that use the Data Service functionalities.

\section{N Body Problem}

In Symphony style NBS, SIs communicate all updates of positions and velocities to the client after each task is received and processed, and then perform a sync at the client. This process is repeated for all generations of tasks. A pseudo-code of this conventional SOA model is given in Appendix C, Algorithm 7.

In ANU-SOAM NBS, tasks for all generations are continuously processed at SIs by accepting sync commands from the client (refer to section 6.1.2). The sync command acts as a barrier between all SIs after each generation of tasks and it compels all SIs to share and update Common Data (positions and velocities) among themselves to reflect the changes for the next generation of tasks.

The ANU-SOAM NBS with Data Service performs well (especially when we consider the compute time curve, which is the result that matters) but not much better than the Symphony style NBS as shown in the Figure 6.6. This is because of the special nature of the NBS naive algorithm. In the naive NBS algorithm all bodies interact with all other bodies in each generation which involves large 


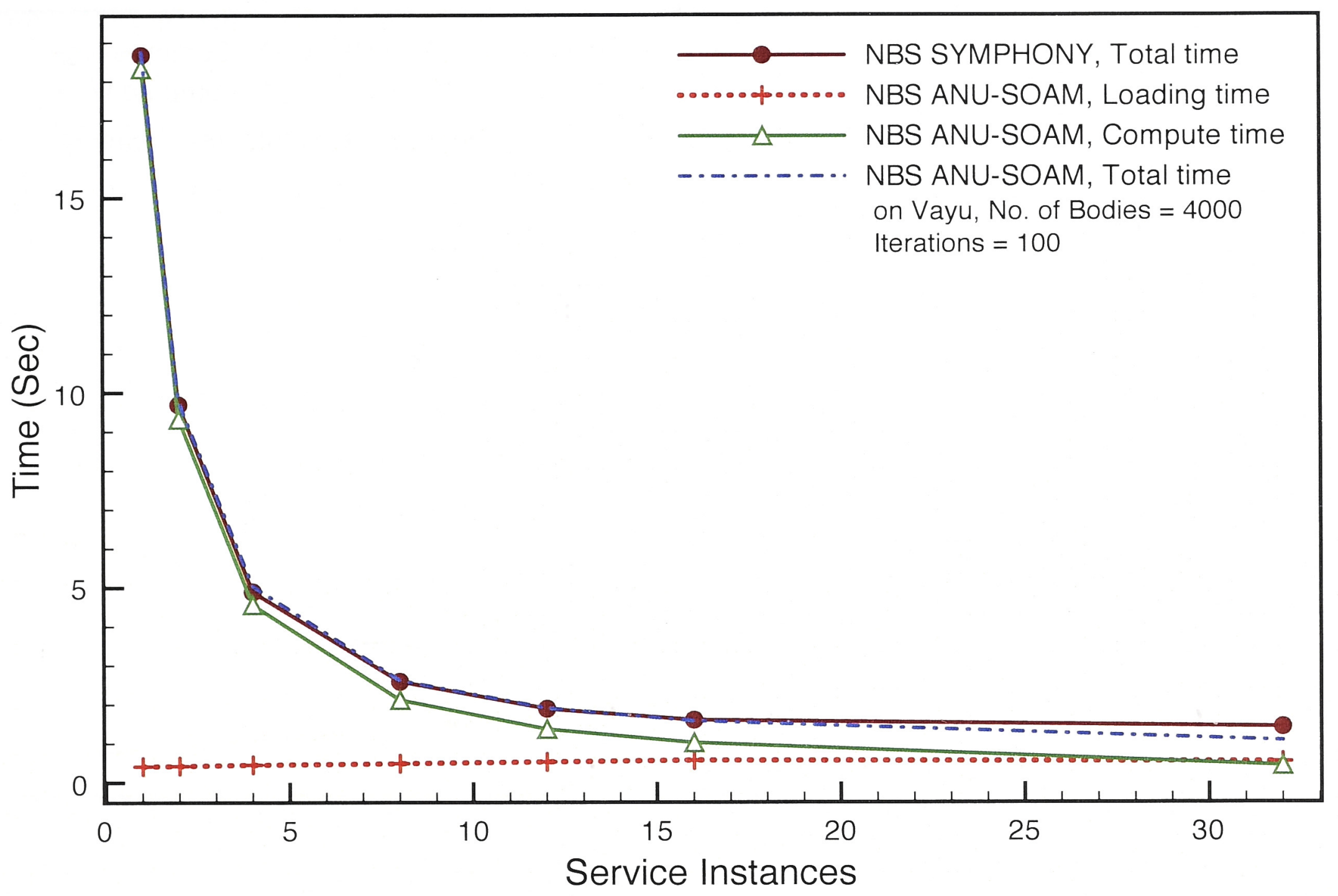

Figure 6.6: ANU-SOAM vs SOA/Symphony Programming Versions: NBS

data transfer after each generation of tasks. The All_Gather_v collective call in ANU-SOAM sync will communicate somewhat similar amount of data as in the gather-broadcast model of the Symphony style application for NBS. The performance advantages from a lower number of interactions between the client and SIs of ANU-SOAM model is less visible, since the large data transfer costs of NBS algorithm impacts heavily both the models in a similar way. However, the contrast between these two styles and the advantages of the ANU-SOAM model for NBS will be more visible in different scenarios like the cloud as we will see in section 6.3.1.

\section{Conjugate Gradient}

The matrix in CDS algorithm can be initiated as a Common Data in both Symphony style and ANU-SOAM style implementations (unlike in NBS), because the matrix remain the same throughout the life of the CGS algorithm. In Symphony style CGS, the matrix-vector multiplication operation is parallelized by sending the vector as task message to all SIs. After each generation of tasks, partial updates of the matrix-vector multiplication operation from all SIs are sent back 


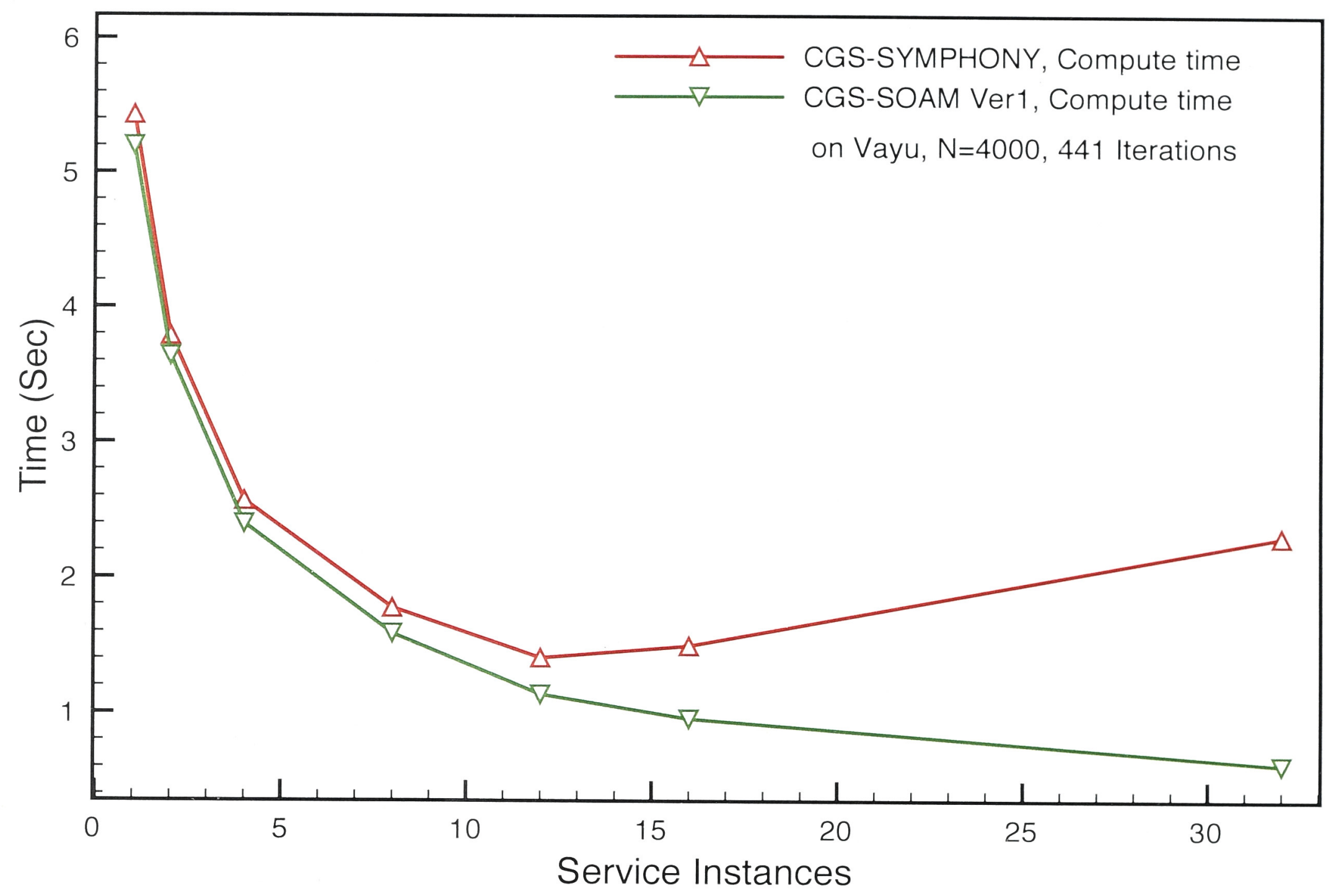

Figure 6.7: ANU-SOAM vs SOA/Symphony Programming Versions: CGS

to the client to synchronize the results there. The variables $\alpha, \beta$, etc are calculated and the residual and other vectors are updated at the client. The updated vector is sent back to SIs (as task messages) for computing next generation of the matrix-vector operations. A pseudo code of this conventional SOA model is given in Appendix C, Algorithm 10.

In ANU-SOAM CGS Version 1, two Common Data arrays are declared - one for the vector and the other for the matrix. The matrix remains the same during the life of the application, whereas SIs can update the vector after processing the parallel computations requested by tasks. The updates are synchronized among SIs after each generation of tasks at the request of the client. This approach allows application programmers to transfer most of the client code to the service side (computation of $\alpha, \beta$, residual and all vector updates in a single tasklet) along with the parallelization of matrix-vector multiplication as we have discussed in in section 6.1.2 on the implementation of ANU-SOAM CGS Version 1.

The results presented in Figure 6.7 show that Symphony style CGS fails to scale after 8 SIs. This is because the increased task communications between the client and SIs were eclipsing the computational advantages in parallelizing 
the application. On the other hand, ANU-SOAM CGS continued to produce good scaling up to 32 SIs. This result shows the advantages of the ANU-SOAM model against the conventional SOA model to deal with these types of scientific applications. Unlike in NBS, large data transfer is not the critical issue in CGS. Rather, it was the increased number of communications between the client and SIs - which arise from the inter-dependency of tasks - that slows down CGS using the conventional SOA/Symphony model. The ANU-SOAM Data Service is able to cut down those communications and thereby costs associated with it to improve the application performance consistently.

Inference Analysis of these results show that the Data Service model adopted in ANU SOAM is superior that of the conventional SOA model for communication intensive and and task interdependent kernels like CGS. It is as efficient as the conventional SOA model for data-transfer intensive kernels like NBS in high performance homogeneous environments. Most importantly we have to note that this was not a comparison between the original Platform Symphony middleware and the ANU-SOAM. ANU-SOAM - with its MPI communication backbone - has already produced high performance results which was not possible with the original implementation of Platform Symphony with its ACE communication system (refer to sections 2.4.2, 4.1.1 and 6.1.2). We were comparing the merits of the ANU-SOAM programming model with that of the conventional SOA model in this section.

\subsubsection{Performance Comparison Against a Native MPI Ap- plication}

Efforts were taken to test the ANU-SOAM NBS application against a native MPI NBS application. A sample MPI NBS application code* is provided by William Gropp et al in their foundational book on OpenMPI - "Using MPI: Portable Parallel Programming with the Message-Passing Interface" [90]. It uses a native pipeline algorithm which uses MPI non-blocking point-to-point communications to rotate the data and All_gather to distribute sizes and displacements of those data sets.

Experiments were conducted for 4000 particles for 100 iterations using ANUSOAM and MPI-Gropp NBS applications. The results show that ANU-SOAM

\footnotetext{
*Available for download at: http://www.mcs.anl.gov/research/projects/mpi/usingmpi/
} 


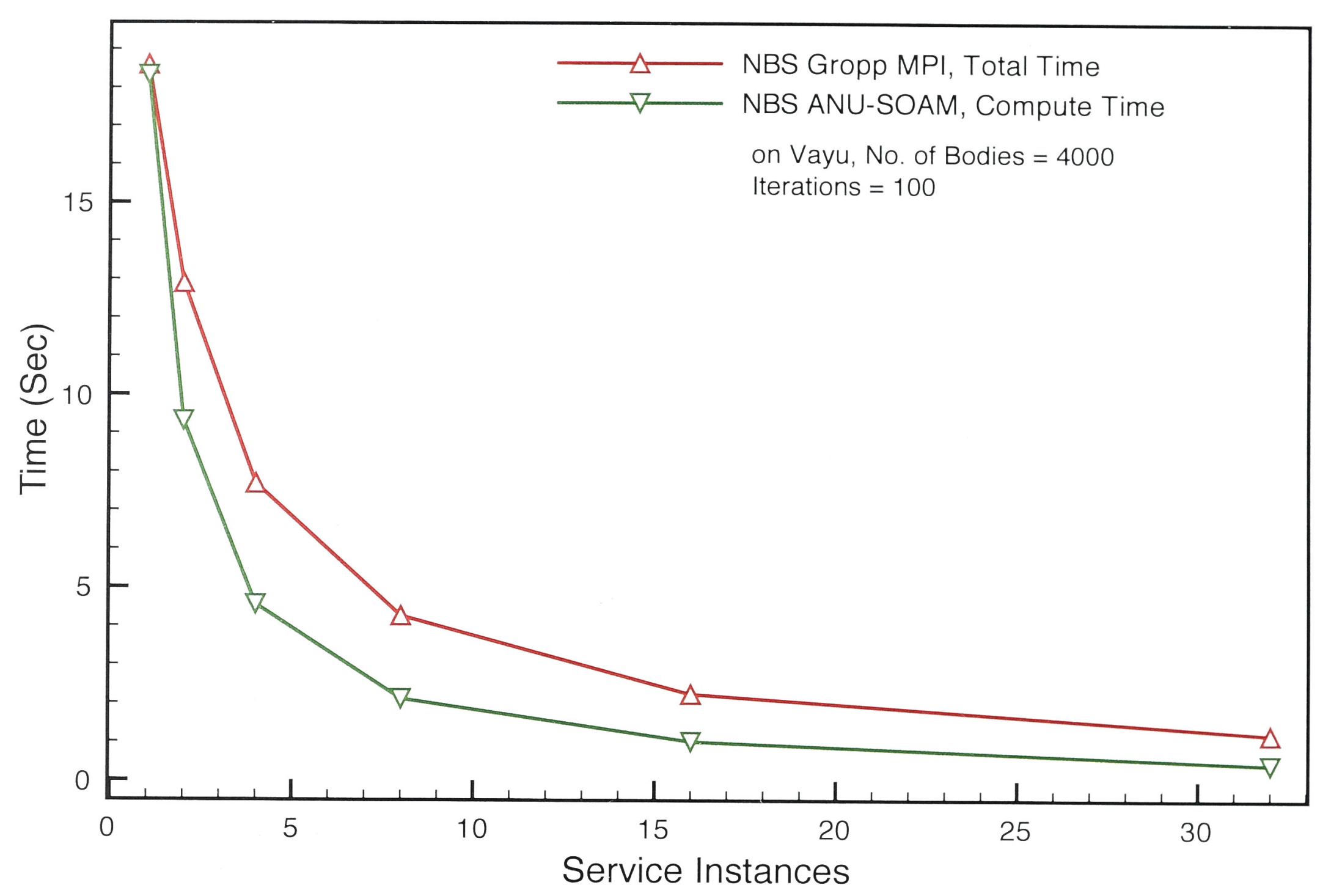

Figure 6.8: ANU-SOAM vs MPI Native (Gropp) Application: NBS

\section{Communication (\% of MPI Time)}

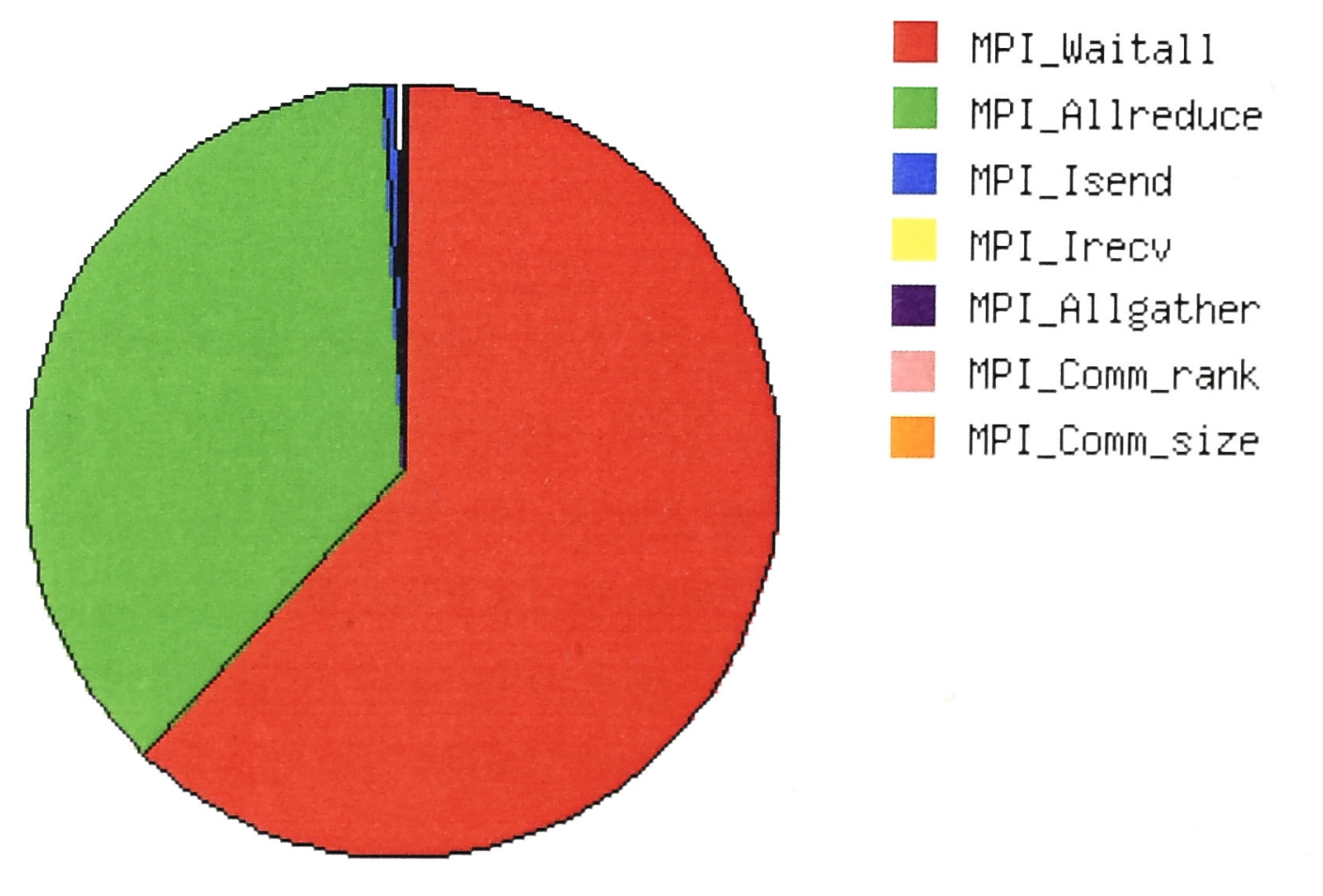

Figure 6.9: MPI (Gropp) NBS: IPM Profile 


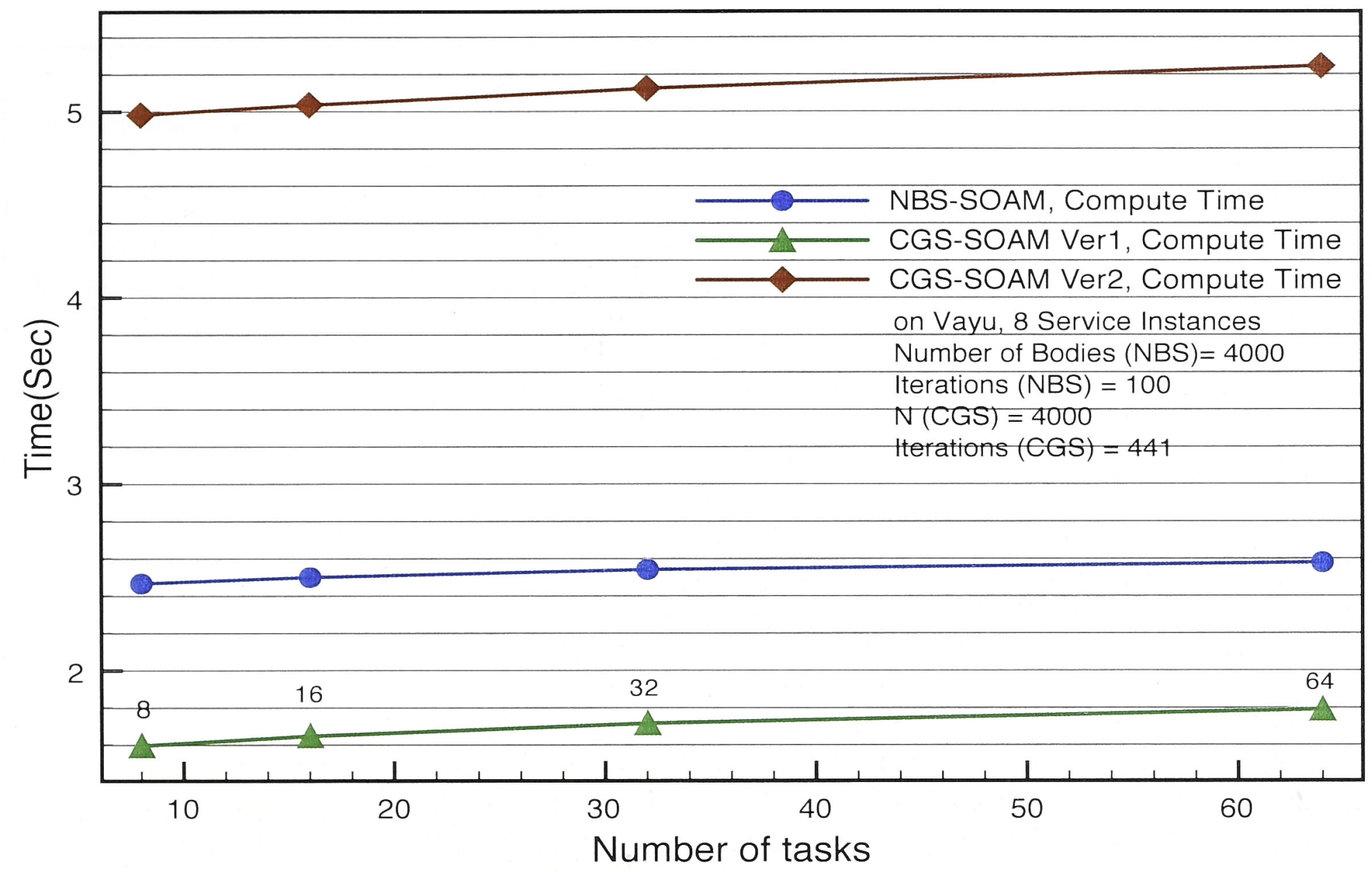

Figure 6.10: NBS and CGS - Varying the Number of Tasks

NBS is performing little better than the Gropp version (refer to Figure 6.8). ANUSOAM uses MPI_All_gatherv to implement its sync function. It was a surprising result. It was noted that All_gather function in MPI - which is used for the sync function in ANU-SOAM - is implemented using efficient algorithms like recursive doubling or Bruck algorithms [137][123]. This may be the advantage of ANUSOAM over the Gropp algorithm which uses a pipeline algorithm with All_reduce collective communications in implementing NBS application. The article [138] also suggests that different internal algorithms of All_reduce may produce below par performances if not used with its advanced properties. An analysis of the Gropp NBS application using the IPM (Integrated Performance Monitoring [139]) profiling tool on Vayu suggests the Gropp implementation is having significant overheads. The profiling results show that the NBS Gropp NBS application spend most of the time on MPI_Waitall which drags its performance down against the ANU-SOAM NBS application (refer to Figure 6.9).

\subsubsection{Effects of Varying Task Numbers}

In a homogeneous environment, we would expect ANU-SOAM to load balance properly when tasks are distributed equally among its SIs (that is when task numbers are equal to multiples of the number of SIs). Experiments were conducted 


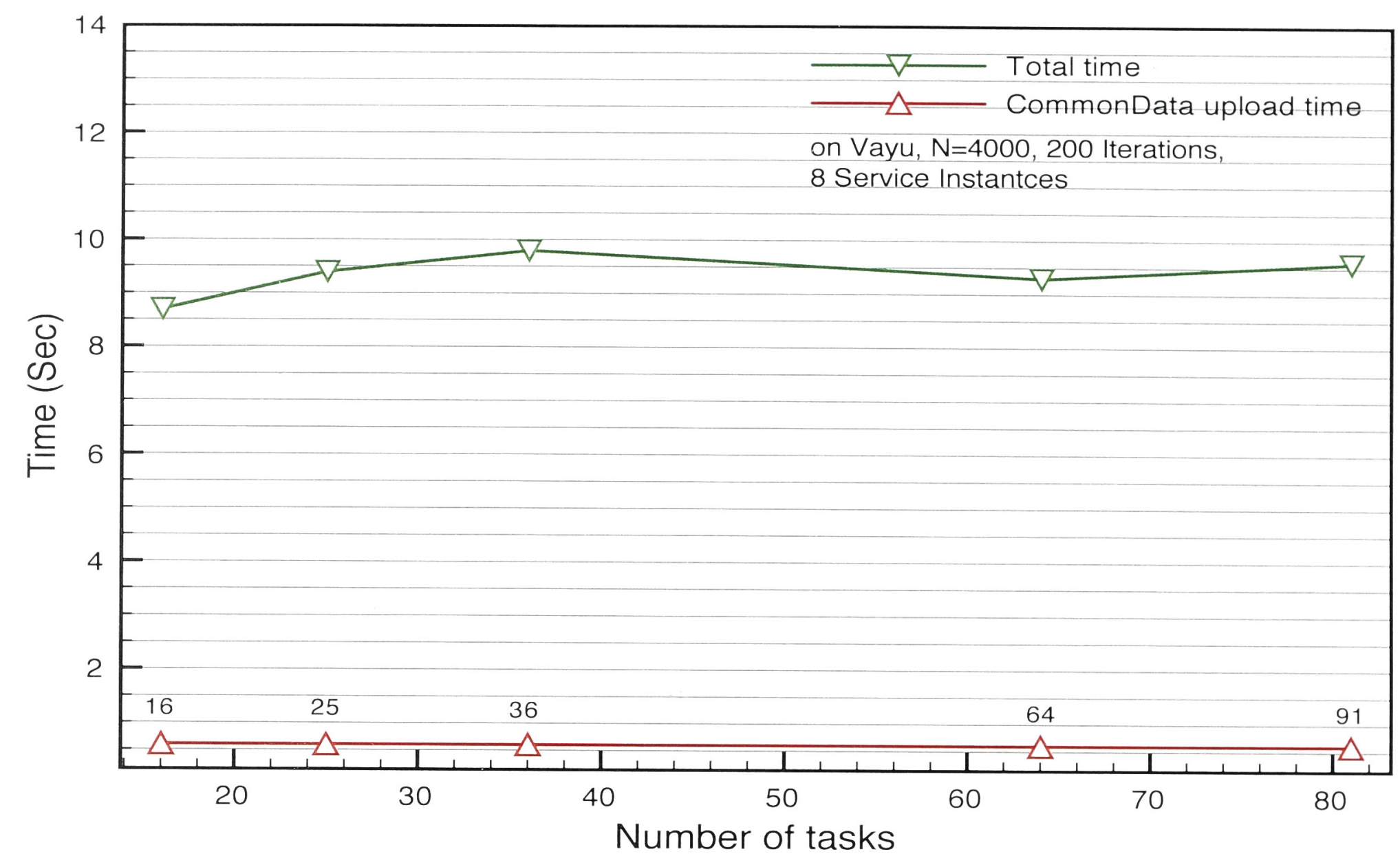

Figure 6.11: HTS - Varying the Number of Tasks

using 8 SIs to verify this assumption on the Vayu cluster. Both NBS and CGS perform fairly well as the task numbers vary from 8 to 64 tasks on 8 SIs (refer to Figure 6.10). There is a slight decrease in performance, which can be attributed to the cost involved in creating and communicating more tasks. However, the increase in compute time is very little and can be neglected.

HTS also performs similarly. The apparent anomalies in HTS with task numbers 25, 36 and 91 are due to improper load balancing. Because of the limitations of the HTS kernel developed for this research, the data matrix can only be partitioned into an equal number of rows and tasks, which means the task numbers are always square of number of rows/columns. However, those experiments with task numbers that are not multiples of 8 (because 8 SIs are used for these experiments), will be poorly load balanced. That is the reason for apparent anomalies in the HTS graph shown in Figure 6.11. CFS was not tested for varying task numbers for it failed to scale beyond 4 SIs, as reported in the previous sections.

Another important observation is that the performance does not improve as the number of tasks increases in any of these experiments. Increased task numbers are used in experiments on heterogeneous environment and this indicate that any performance improvement achieved in those experiments cannot be attributed to increased task numbers, but only to the merit of load balancing techniques used 


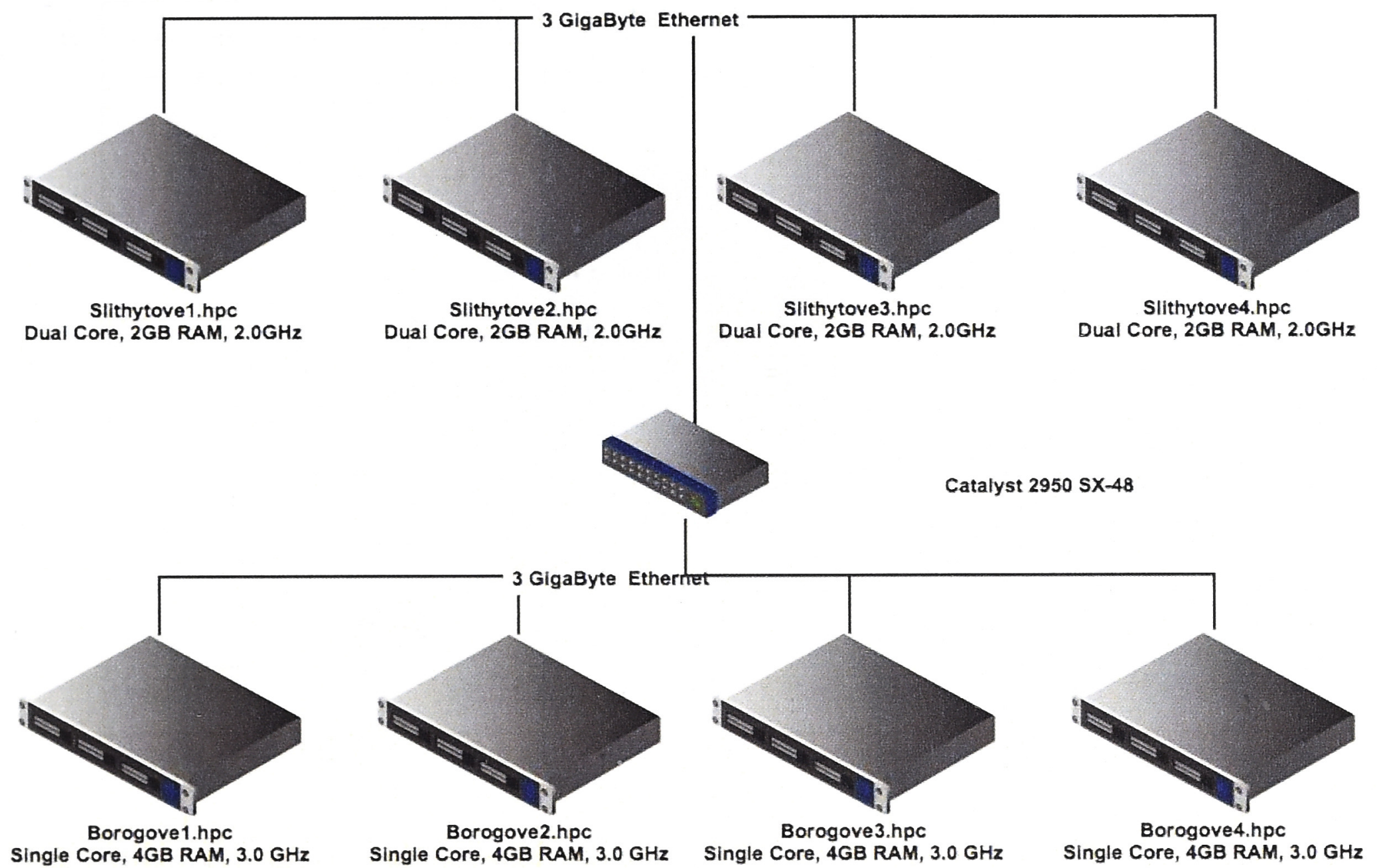

Figure 6.12: ANU CSIT Cluster: Heterogeneous Environment

in ANU-SOAM.

\subsection{Heterogeneous Environment}

\subsubsection{Heterogeneous Experimental Environment}

The heterogeneous experiments were conducted on selected machines at ANU CSIT cluster (refer to Figure 6.12). The fast machines (named Borogoves 1 to 4) have AMD Phenom II X4 single core cpu machines running Debian 6 Squeeze with $4 \mathrm{~GB}$ memory and $3.0 \mathrm{GHz}$ processor speed. The slow machines (named Vorpalblades 1 to 4) have AMD Athlon 64 X2 dual core cpu machines running Ubuntu 8 Hardy with 2 GB memory and $2.0 \mathrm{GHz}$ processor speed. They are networked together using a 3 GigaByte Ethernet switch.

\section{Relative Performance of Machines}

Independent experiments were conducted on each machine of the Borogove and Slithytove cluster using ANU-SOAM NBS kernel and the relative performance of 


\section{Relative Performance of Machines}

NBS

$N=2000$

100 Iter

Total time taken

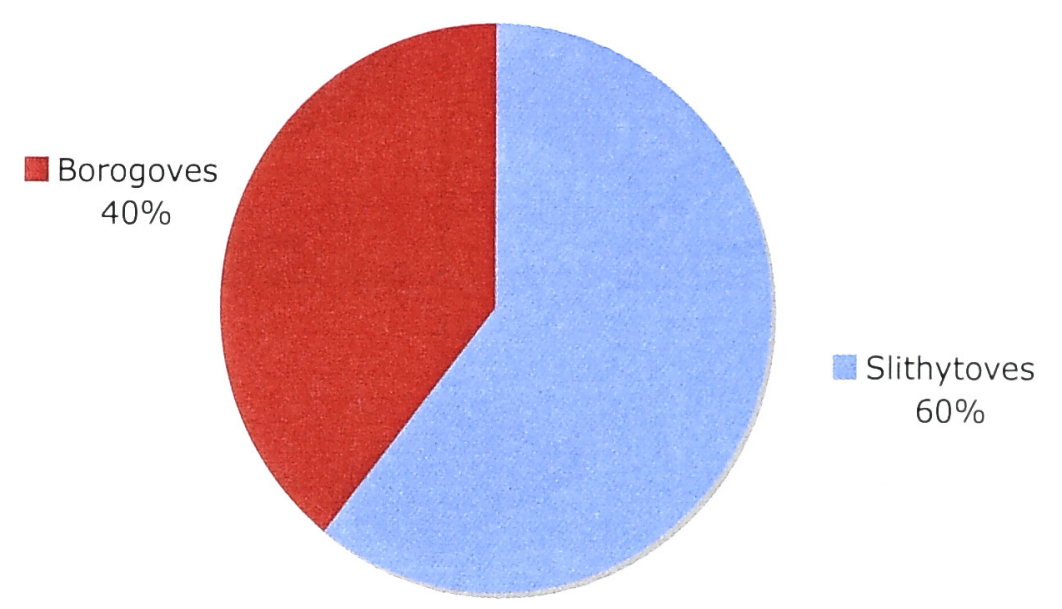

Figure 6.13: ANU CSIT Cluster: Relative Performance of Machines

two sets of machines are collected. The results are expressed in Figure 6.13. The ratio of total time taken $(40 \%-60 \%)$ to complete the NBS between Borogoves and Slithytoves can be converted to a performance ratio of 3:2 between them with was further rounded to 2:1 for convenience.

\subsubsection{Performance Analysis of Load Balancing Efforts}

In order to understand the effect of using load balancing capabilities of ANUSOAM, the four scientific kernels discussed earlier were experimented in this heterogeneous environment with and without load balancing enabled. Load balancing is activated by defining "weighted round robin" as the load balancing policy in RM using the client API. A policy ration of 2:1 is (manually) fed to RM which in turn distributes tasks among the compute nodes using the same ratio among the Borogoves and Vorpalblades. For experiments without load balancing, the resource manager policy is set to "round robin", and the default policy ratio is $1: 1$.

\section{N Body Problem}

Figures 6.14 and 6.15 present the result of NBS experiments. Figure 6.14 presents the comparison of NBS with and without load balancing, using varying data sets. The number of elements to be processed (the data set) was increased from 1000 to 


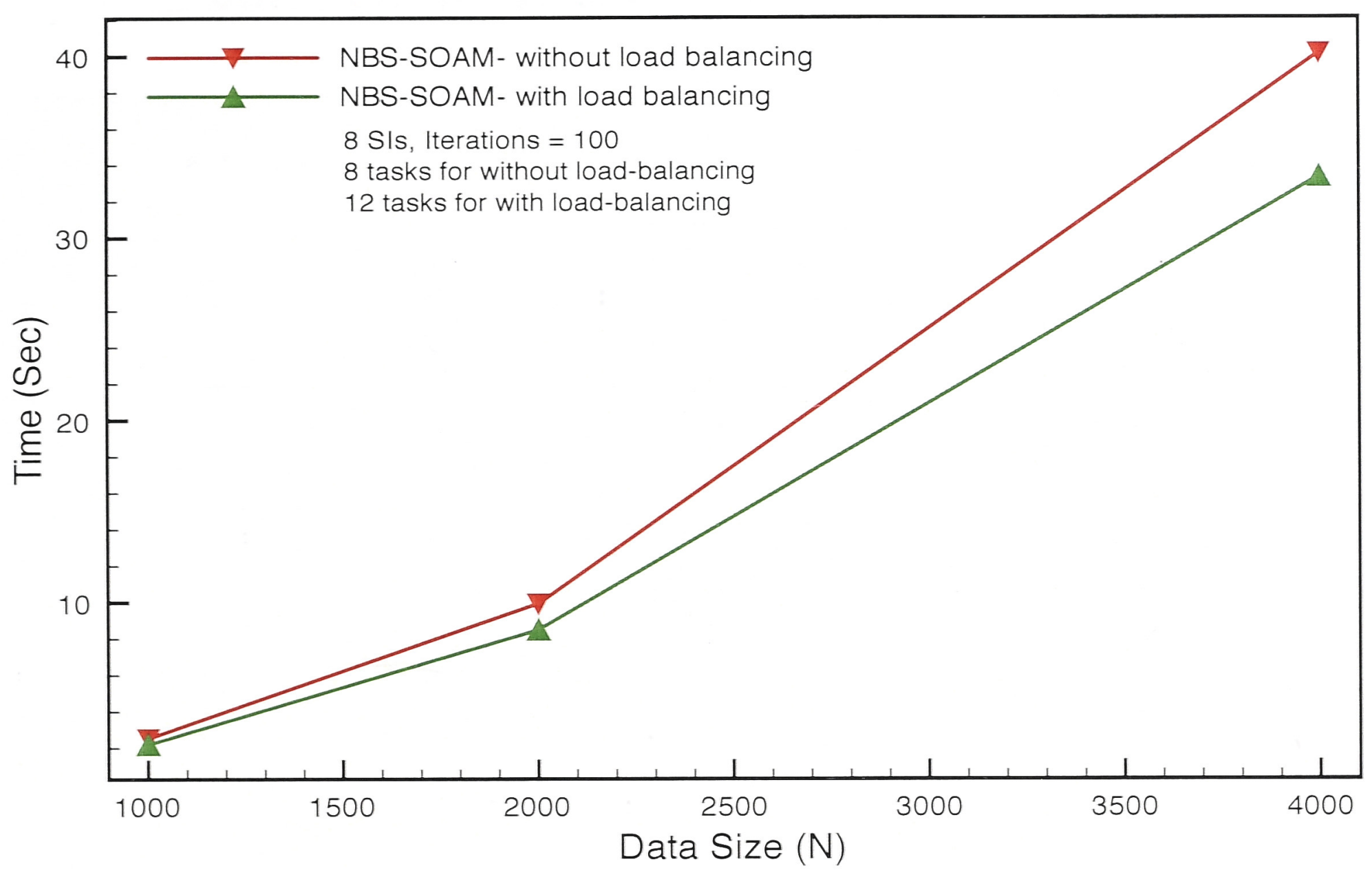

Figure 6.14: NBS on Heterogeneous Environment

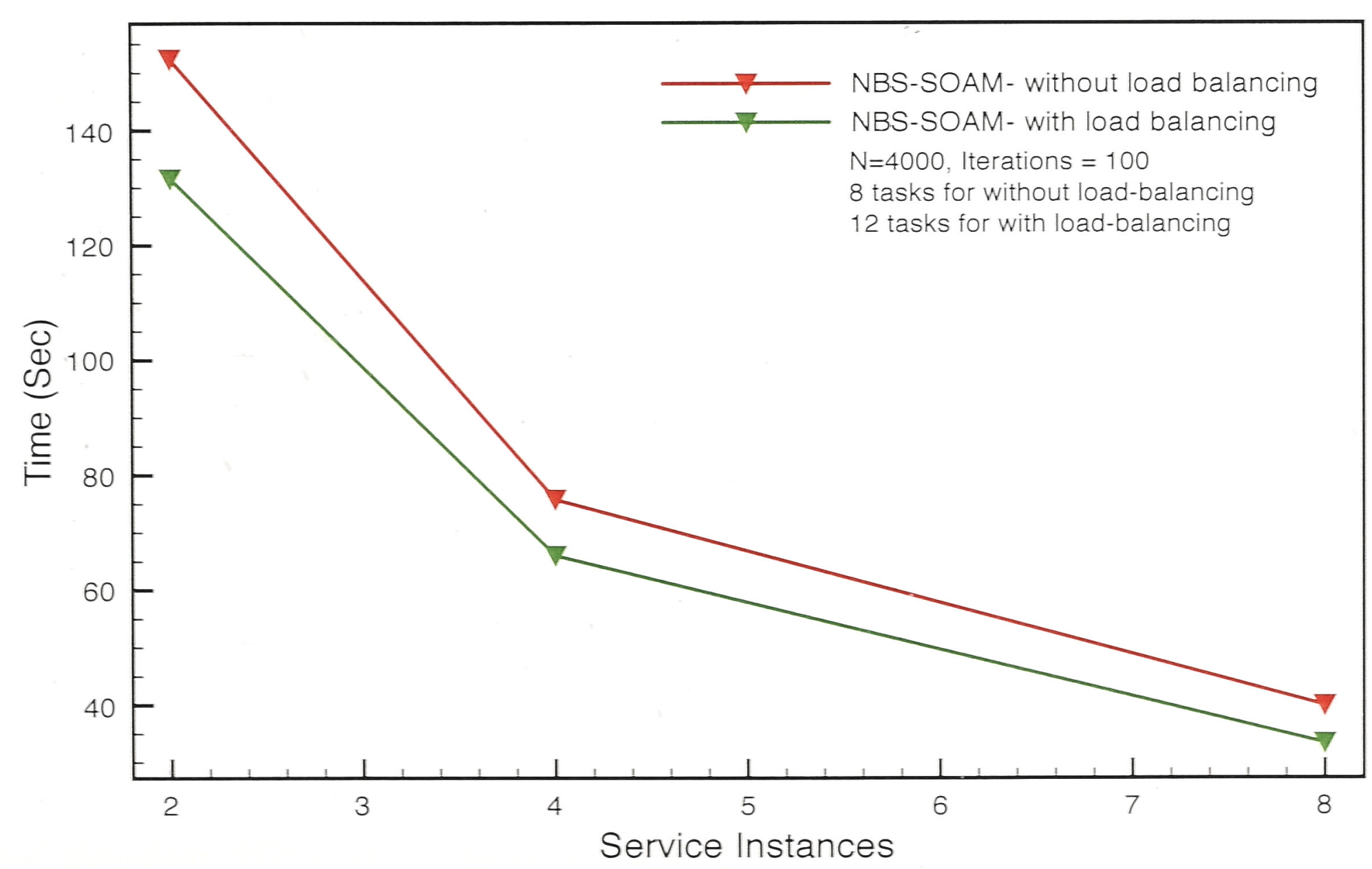

Figure 6.15: NBS Scaling on Heterogeneous Environment 


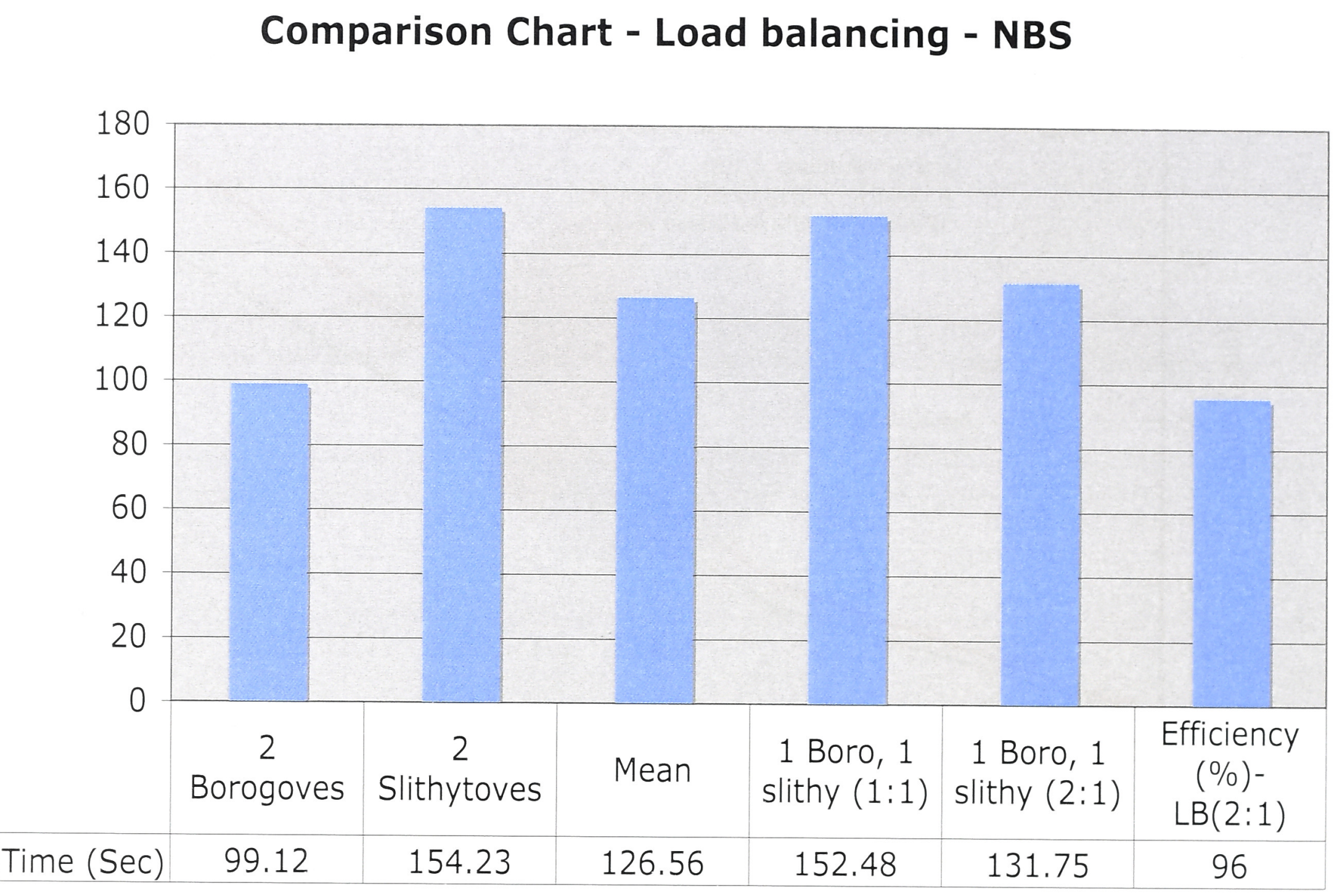

Figure 6.16: NBS on Heterogeneous Environment: Performance Comparison

4000. The results show that when load balancing was enabled the performance improved consistently and proportionately over varying data sets. There is roughly $20 \%$ performance improvement when load balancing is enabled in ANU-SOAM for NBS using 8 SIs.

Figure 6.15 presents the scalability with load balancing and compare it compares it to the same results without load balancing. The data set remained the same during the experiments (4000) but the number of SIs changed from 2 to 8. In all scenarios load balancing gives better results. It is also evident that as the number of SIs increase we get proportionately lower performance on ANU-SOAM with load balancing than with reduced SIs. This is in line with what we would expect because as the number of SIs increase, slower machines could compel faster machines to wait on them more often.

Number of Tasks The number of tasks used in experiments without load balancing were 8 which is equivalent to the number of SIs. For experiments with load balancing 12 tasks were used because the policy ratio of 2:1 among 4 Borogoes and 4 Slithytoves would imply at least 12 tasks to properly load balance 


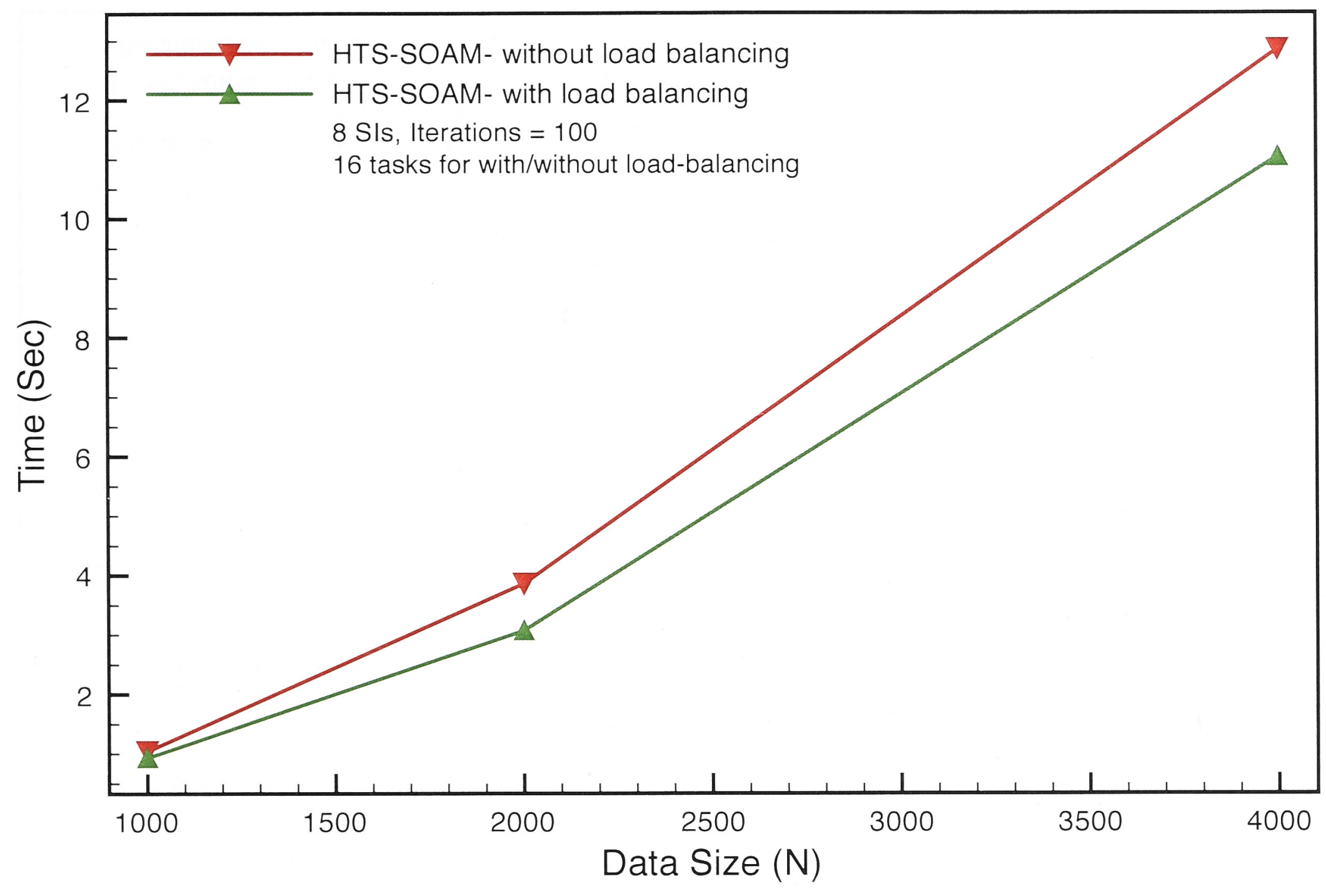

Figure 6.17: HTS on Heterogeneous Environment

tasks between these machines. However, the increasing number of tasks is not a contributing factor in the increase in performance of these experiments as we have discussed in section 6.1.5.

Performance Comparison Another interesting question investigated was how good is the load balancing mechanism of ANU-SOAM comparing to the theoretical projections. In order to answer this question, experiments were conducted separately on Borogove (faster) and Slithytove (slower) clusters. From those results the mathematical average of performance in grouping these two clusters in an ideal world was derived. This mathematical average was compared against the actual results we got on a mix of these two cluster machines.

Experiments were conducted with NBS using a data size of 4000 for 100 iterations, using two SIs and results are presented in Figure 6.16. Two Borogove machines were $155 \%$ times faster than two Slithytove machines in computing NBS and the mathematical average of combining those two is calculated as 126.56 seconds. From the graph shown in Figure 6.16, it becomes evident that when the load balancing was enabled using 2:1 policy ratio between a Borogove and a 


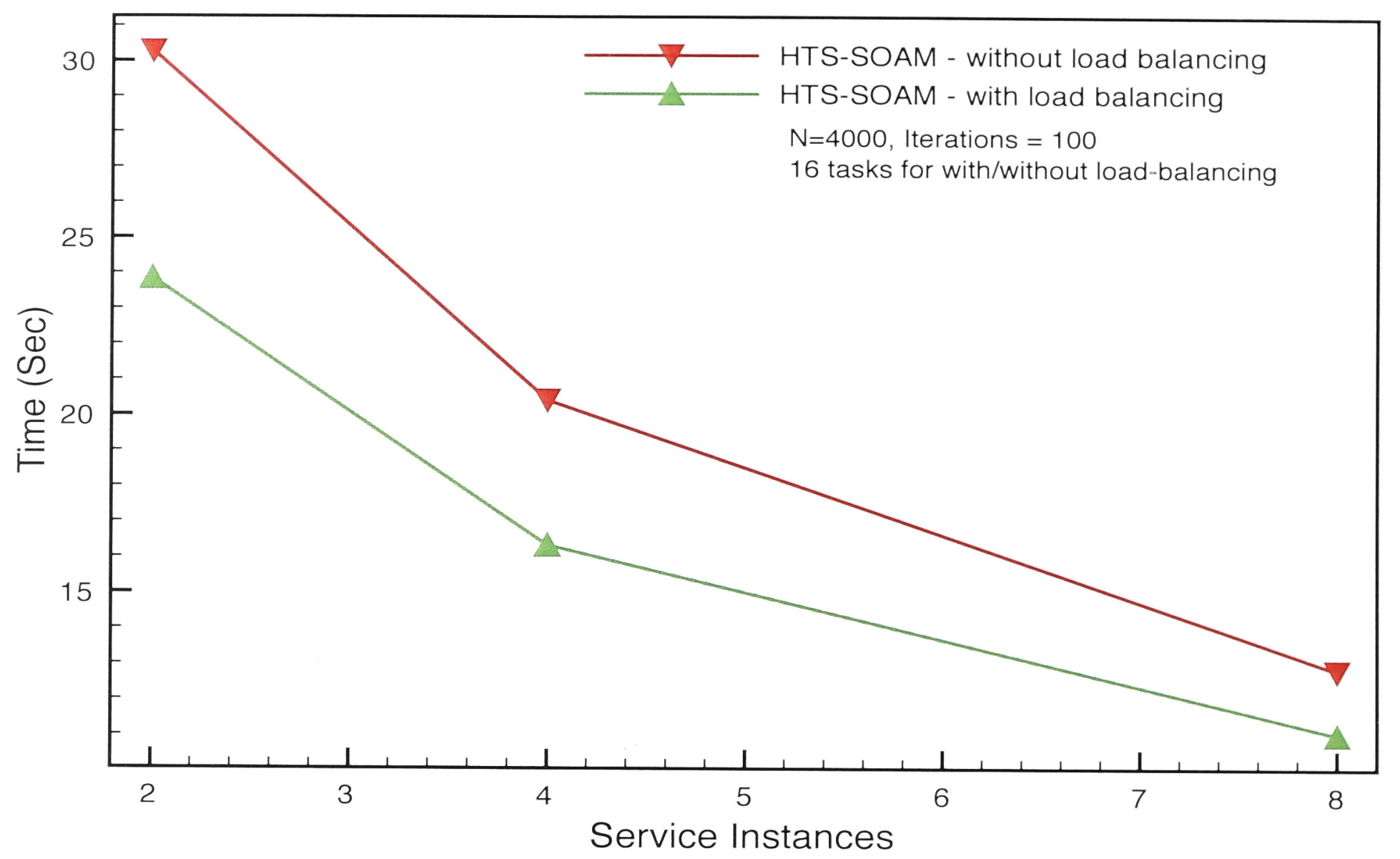

Figure 6.18: HTS Scaling on Heterogeneous Environment

Slithytove machine, ANU-SOAM completed the computation in 131.75 seconds. That means the ANU-SOAM load balancing technique produced $96 \%$ efficiency compared to the expected theoretical result in this experimental heterogeneous condition.

\section{Heat Transfer Equation}

Figure 6.17 presents the comparison of HTS with and without load balancing, with varying data sets. Since the data is divided into near-cube subsets (refer to section 5.2), the number of tasks for 8 SIs in all cases remains at 16 (4 rows*4 columns =16). This limitation of the algorithm brings a load imbalance for experiments with load balancing, where we need 12 tasks or multiples of 12 tasks. The result shows that HTS still performs well with load balancing turned on than off and the performance improvement is also around $20 \%$. It shows that the Borogoves are capable of handling more tasks than the slower Slithytoves, as we expected.

Figure 6.18 presents the scalability with load balancing and compare it compares it to the same results without load balancing. The data set remained the same during the experiments (4000) but the number of SIs changed from 2 to 8. As in the case of NBS, the load balancing produce better results in all scenarios 


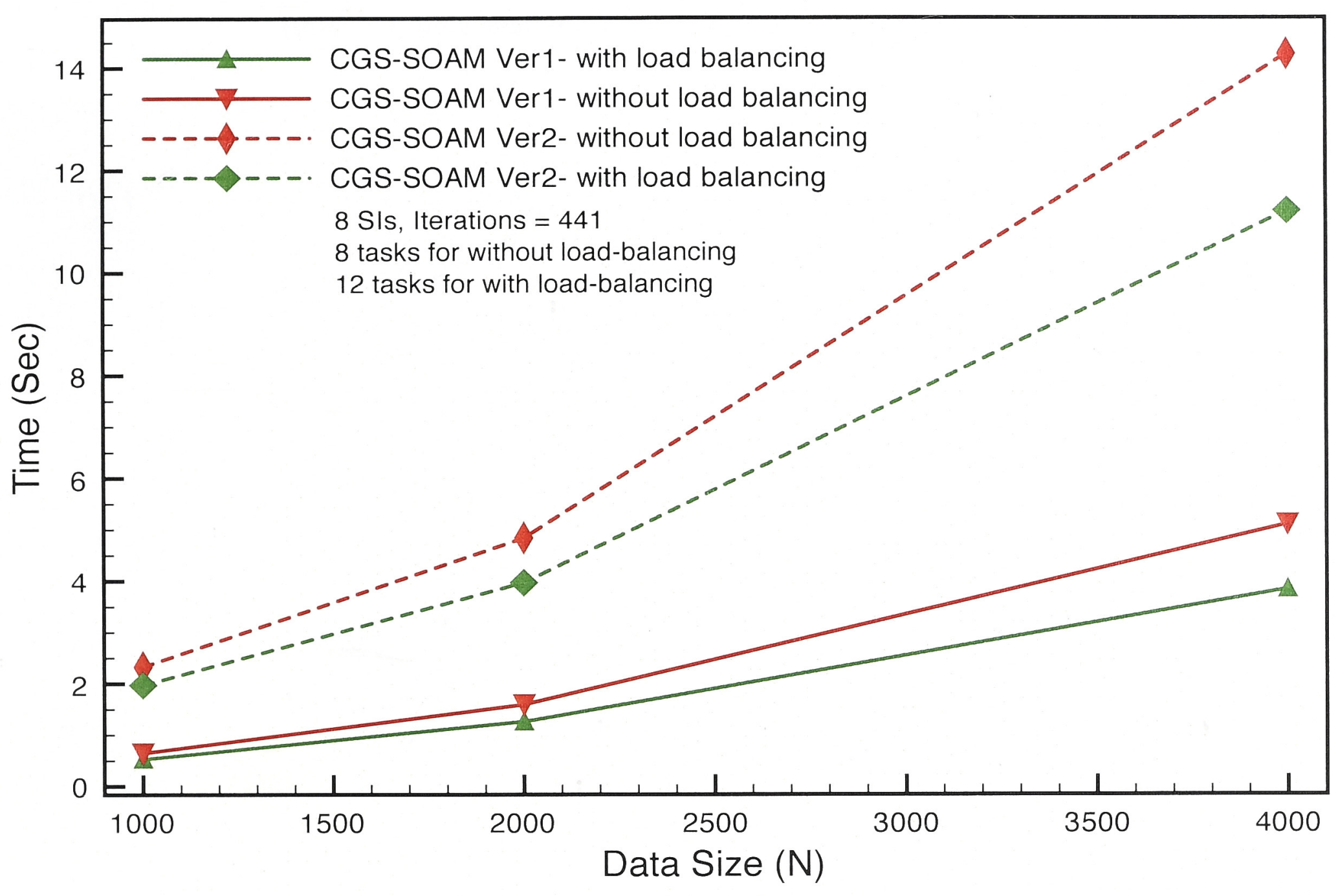

Figure 6.19: CGS on Heterogeneous Environment

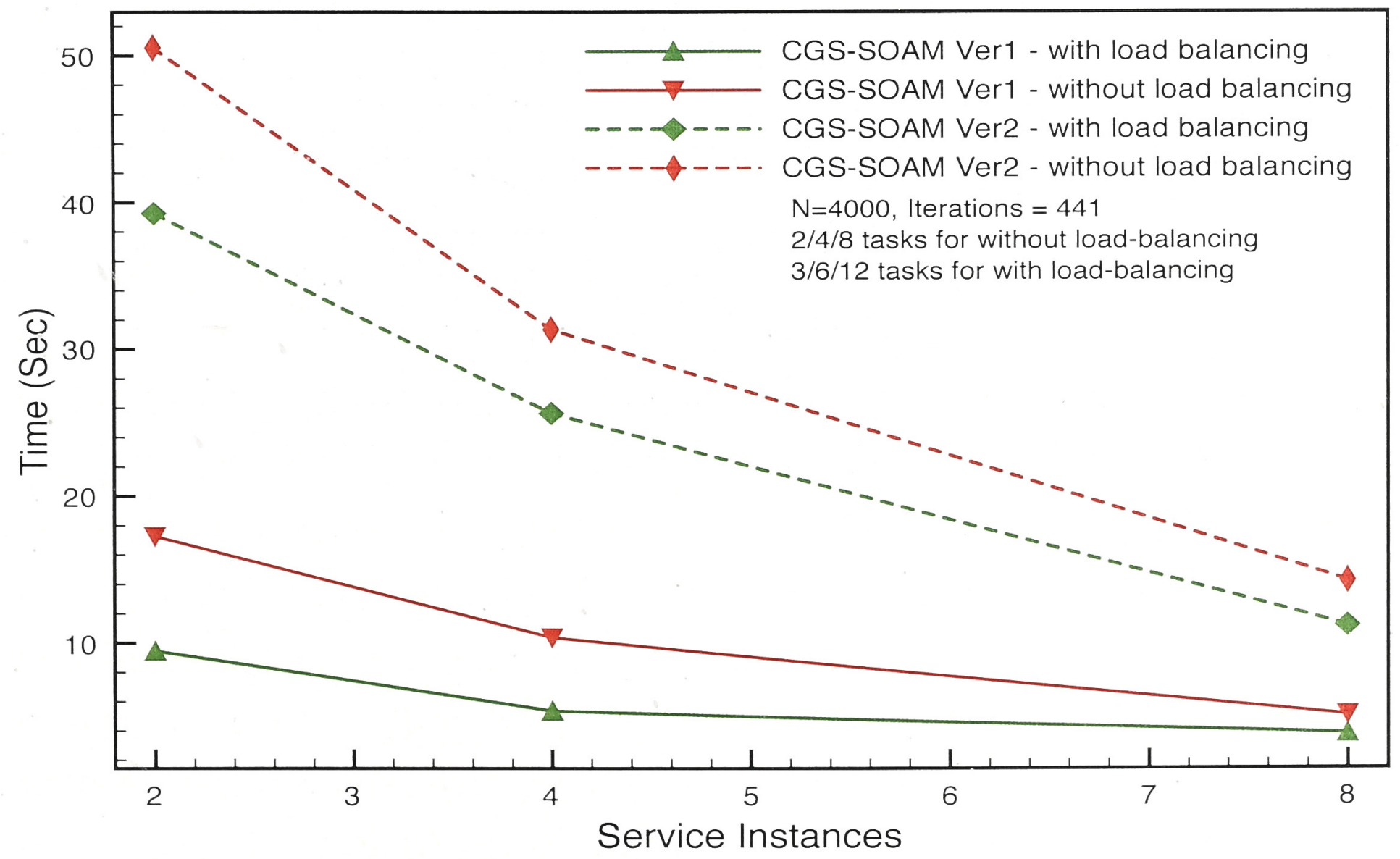

Figure 6.20: CGS Scaling on Heterogeneous Environment 


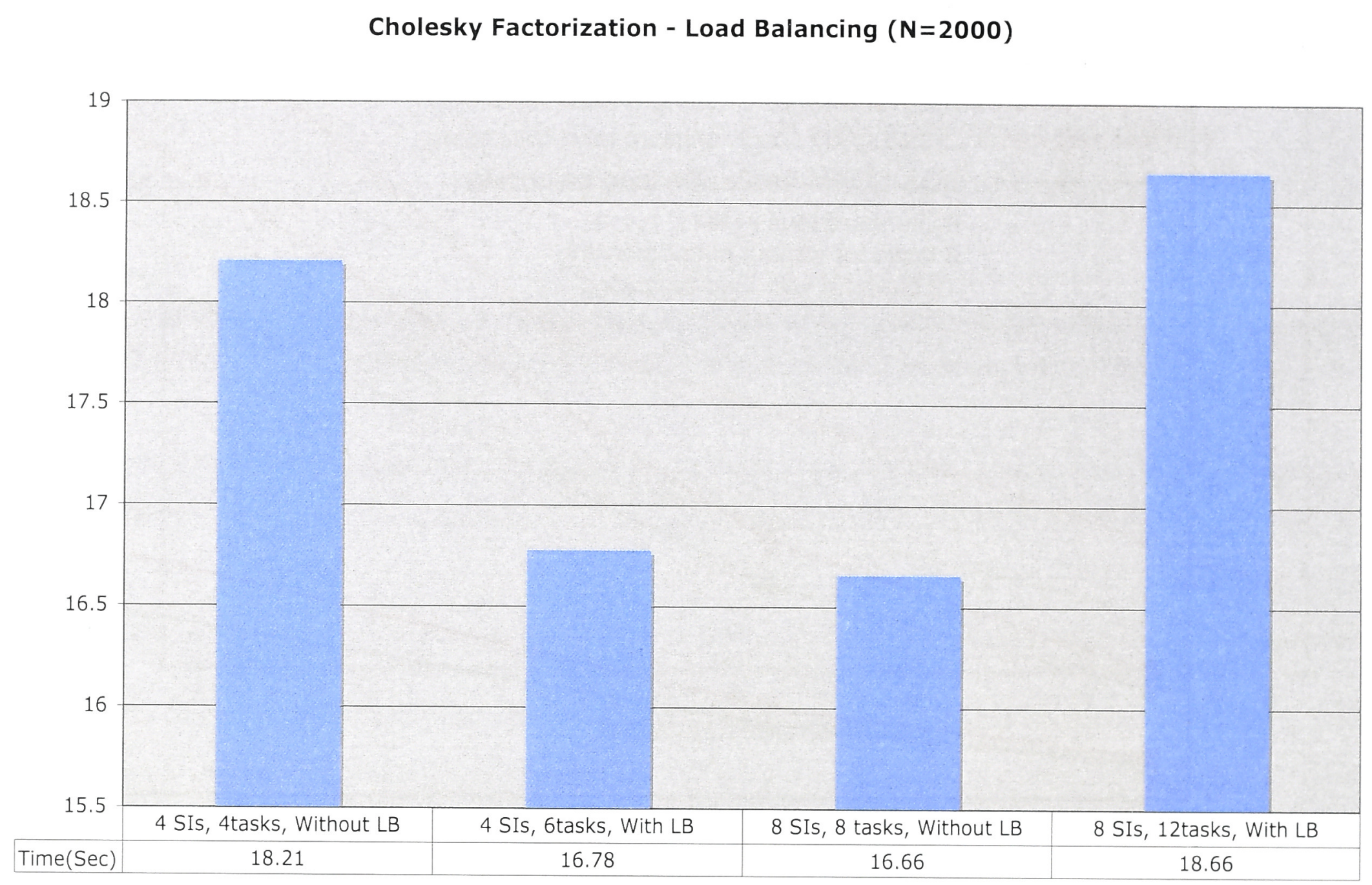

Figure 6.21: CFS on Heterogeneous Enviornment

but lesser number of SIs take more time to finish the work, which is quite an obvious outcome.

\section{Conjugate Gradient}

Two versions of Conjuagte Gradient have been tested for performance under heterogeneous conditions. The advanced version (Version 2), as explained in section 5.3.1, parallelises not only the matrix-vector operation, but also the vectorvector operations whose task granularity turned out to be not that optimum (or too small). The other version of CGS (Version 1 as discussed in section 6.1.2) parallelises only the matrix-vector operation which has a more optimum task granularity. Both these versions perform better with the ANU-SOAM load balancing function on heterogeneous conditions. The graph 6.19 shows that with 8 SIs and for varying matrix sizes, load balancing technique produce a little over $20 \%$ performance improvement, for both versions of CGS.

Figure 6.20 presents the scalability with load balancing and compare it compares it to the same results without load balancing. The data set remained the same during the experiments (4000) but the number of SIs changed from 2 to 8. 
As in the case of NBS and HTS, the load balancing produce better results in all scenarios but lesser number of SIs take more time to finish the work.

\section{Cholesky Factorization}

Results using CFS in a heterogeneous environment is different to that of all other experiments discussed above, but still consistent with the results we have received previously in homogeneous conditions. The highly refined CFS kernel (Version 2) on ANU-SOAM was used for experiments and scales well up to 4 SIs in homogeneous conditions but not beyond that. The reasons for this behaviour can be explained with the results in section 6.1.2. Section 6.1.2 suggests that we experience performance improvement for CFS only up to 4 SIs, but beyond that it may not scale. In a similar way, the load balancing results of CFS deteriorates beyond 4 SIs (refer to Figure 6.21). As explained in section 6.1.2, since the task granularity is already too small to be optimum in the refined version (Version 2) of CFS kernel, any increase in task numbers and thereby overheads involved in reduced task granularity will outweigh any performance advantage gained from load balancing efforts. In order to take full advantage of load balancing we need at least 12 tasks with 8 SIs in the experiments we conducted, which is up 4 tasks used for experiments without load balancing. This could also contribute to this performance anomaly beyond 4 SIs in the CFS implementation.

\subsection{Cloud Environment}

The experiments were conducted to evaluate the deployability of ANU-SOAM in an IaaS cloud and also to test its performance under various conditions. The experiment was based on the presumption that ANU-SOAM - as an application middleware - should be able to function as a PaaS hosted on an IaaS cloud as explained in section 4.4 and section 2.3. The cloud settings were arranged and experiments were conducted by Mr Boby Jose Thekkanath, as part of an extended project of this research work (ANU research programmer, position number: 5089913/17282) with my guidance and assistance.

\subsubsection{Cloud Experimental Environment}

Two types of cloud experimental scenarios were envisaged. The first one was to run ANU-SOAM within a public cloud IaaS. That is, both the client and SIs to 


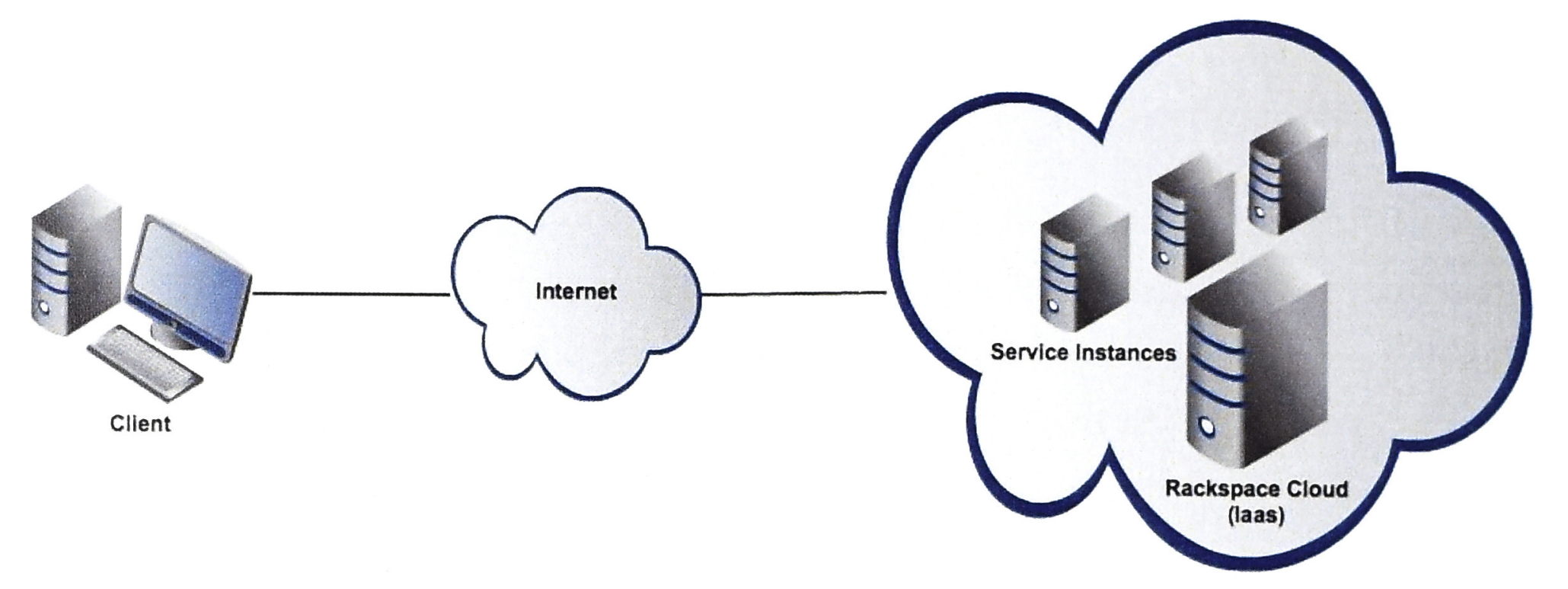

Figure 6.22: ANU-SOAM: Cloud over the Internet

run within a public cloud IaaS. The second one is to access a public cloud IaaS across Internet, using ANU-SOAM. That is to run the client from a home PC and access the cloud computing resources over the Internet by running the SIs in a cloud (refer to Figure 6.22). The assumption is that SIs within the cloud interact with each other internally.

Selection of the right cloud provider turned to be a critical issue, for ANUSOAM uses OpenMPI as its communication backbone which does not support Network Address Translation (NAT). Since the Amazon cloud uses NAT to translate the public IP addresses of its compute nodes [140], Rackspace (which doesn't use NAT technology) was chosen to deploy ANU-SOAM, especially to enable the second set of experiments.

A Rackspace cloud node of Quad-Core AMD Opteron Processor 2374 HE with $4 \mathrm{~GB}$ memory is used for the experiments. For experiments over the Internet, the client was run on a home PC and accessed the cloud service using ADSL $2+$ provided by a commercial Internet Service Provider with a maximum of 1.5 Mbps speed from a heavy traffic geographical region.

All experiments were conducted on a single node for up to 4 Service Instances running on it. There is a reason why the experiments were limited to a single node on Rackspace. To access Rackspace nodes from a home PC, we have to use the public IP addresses of the Rackspace nodes in ANU-SOAM. This is because ANU-SOAM uses OpenMPI as its communication backbone, which in turn reads the IP addresses of the host machines from a client 'hostfile'. Using more than a single Rackspace node would cause the nodes to communicate with each other 


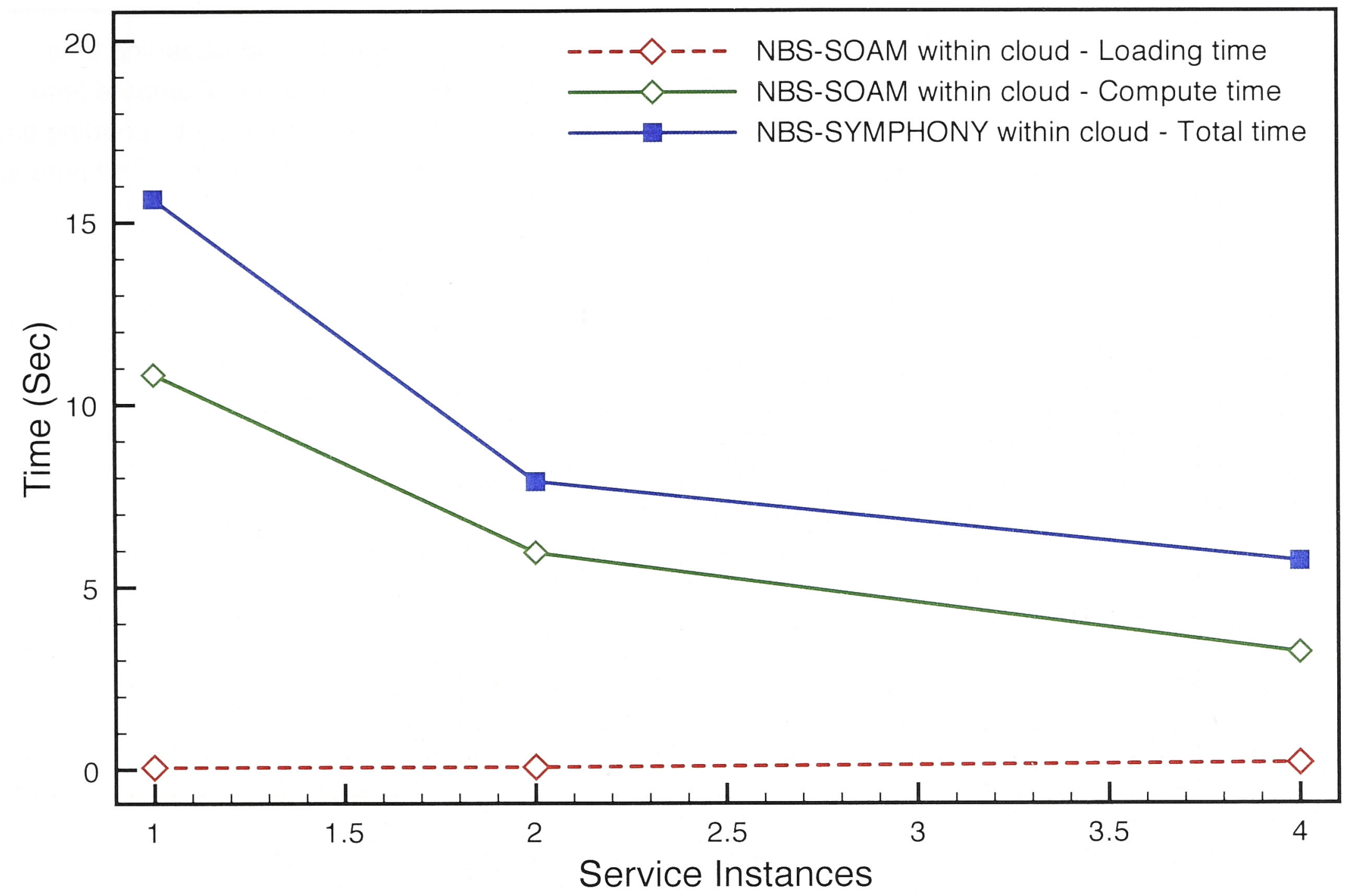

Figure 6.23: NBS SOAM \& Symphony Versions within the Rackspace Cloud

over the Internet, rather than taking advantage of the internal IP addresses which are networked using fast interconnects.

This research is interesting in a scenario where the SIs interact with each other within the cloud, but a client may sit outside to access those service resources at runtime (refer to Figure 6.22). In this scenario, it can be tested realistically only if there is only one node, which has as many SIs that can run on it. From experience we decided that the maximum number of SIs that can run on a single Rackspace cloud machine without compromising performance would be 4 .

We could have solved this problem if we were able to provide a separate hostfile with internal hostnames of SIs to the OpenMPI communicator connecting SIs alone. But there is no provision in OpenMPI - at this stage - to supply a separate hostfile to this communicator. This is a limitation of OpenMPI.

Cloud experiments were conducted for both the conventional SOA and ANUSOAM versions of NBS kernel. Since the Data Service is absent in the conventional SOA/Symphony version, SIs have to communicate the results back to the client after computing the workload of each task (refer to section 6.1.3). In ANUSOAM NBS, tasks can update Common Data in the Data Service (at service 


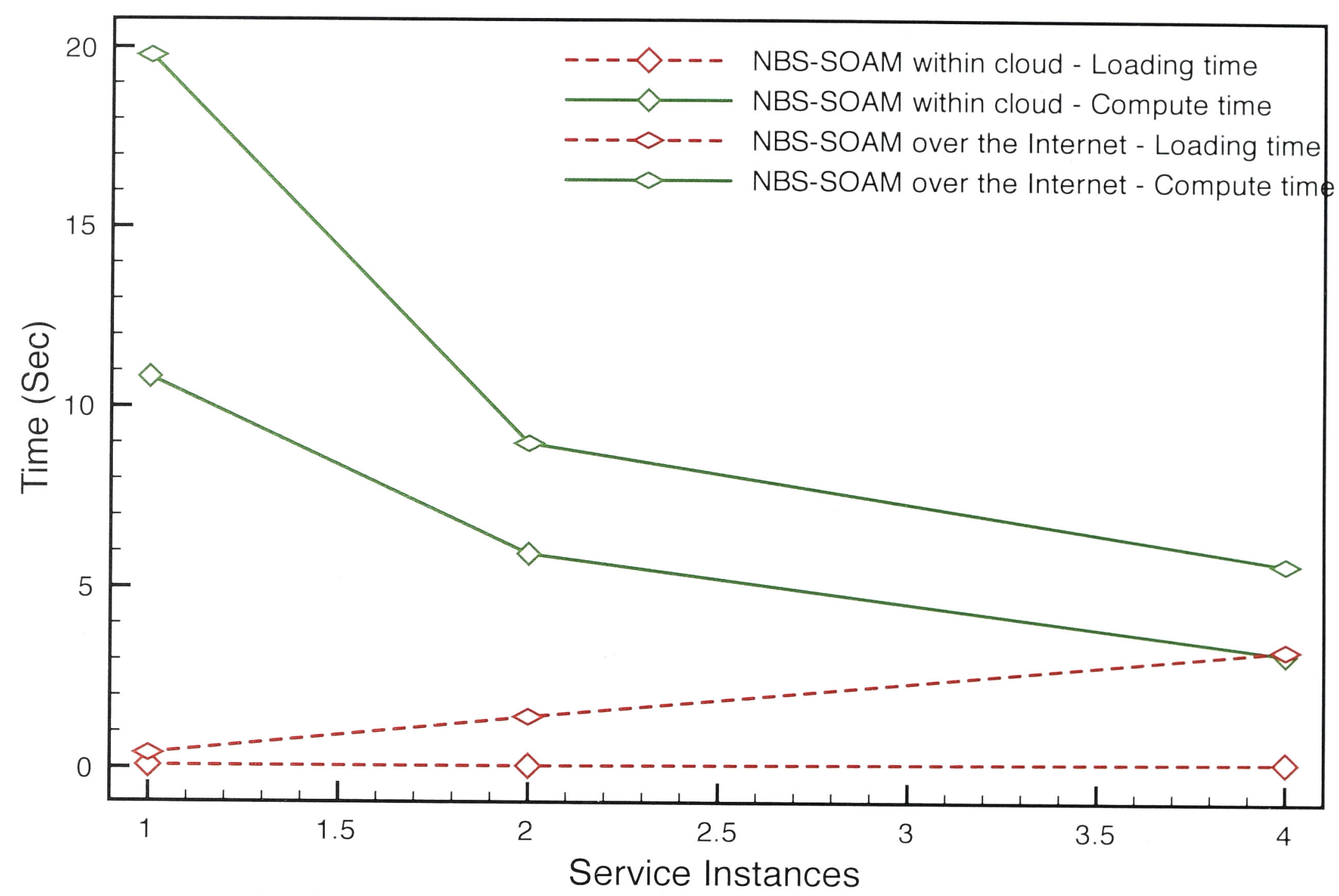

Figure 6.24: NBS SOAM Version within Cloud \& over the Internet

side) and continue to move to next generations without communicating back to the client. By comparing these two versions, we aim to understand the effects of Data Service (and thereby the effects of ANU-SOAM architecture) in a cloud environment. A data set of 2000 bodies and 100 iterations were used for all these experiments.

\subsubsection{Performance within Cloud}

The graph shown in Figure 6.23 presents the results of Symphony and ANUSOAM versions of NBS running within the Rackspace cloud. Both versions performs fairly well in this scenario. The ANU-SOAM version performs with comparable or even better results to that shown on the Vayu cluster in this case (refer to Figure 6.1). The minimal Common Data loading time can be attributed to the fact that the client and SIs run on the same machine in this case. It naturally reduces loading time as compared to the previous cases (in heterogeneous experiments) where separate machines were used to host each SI. 


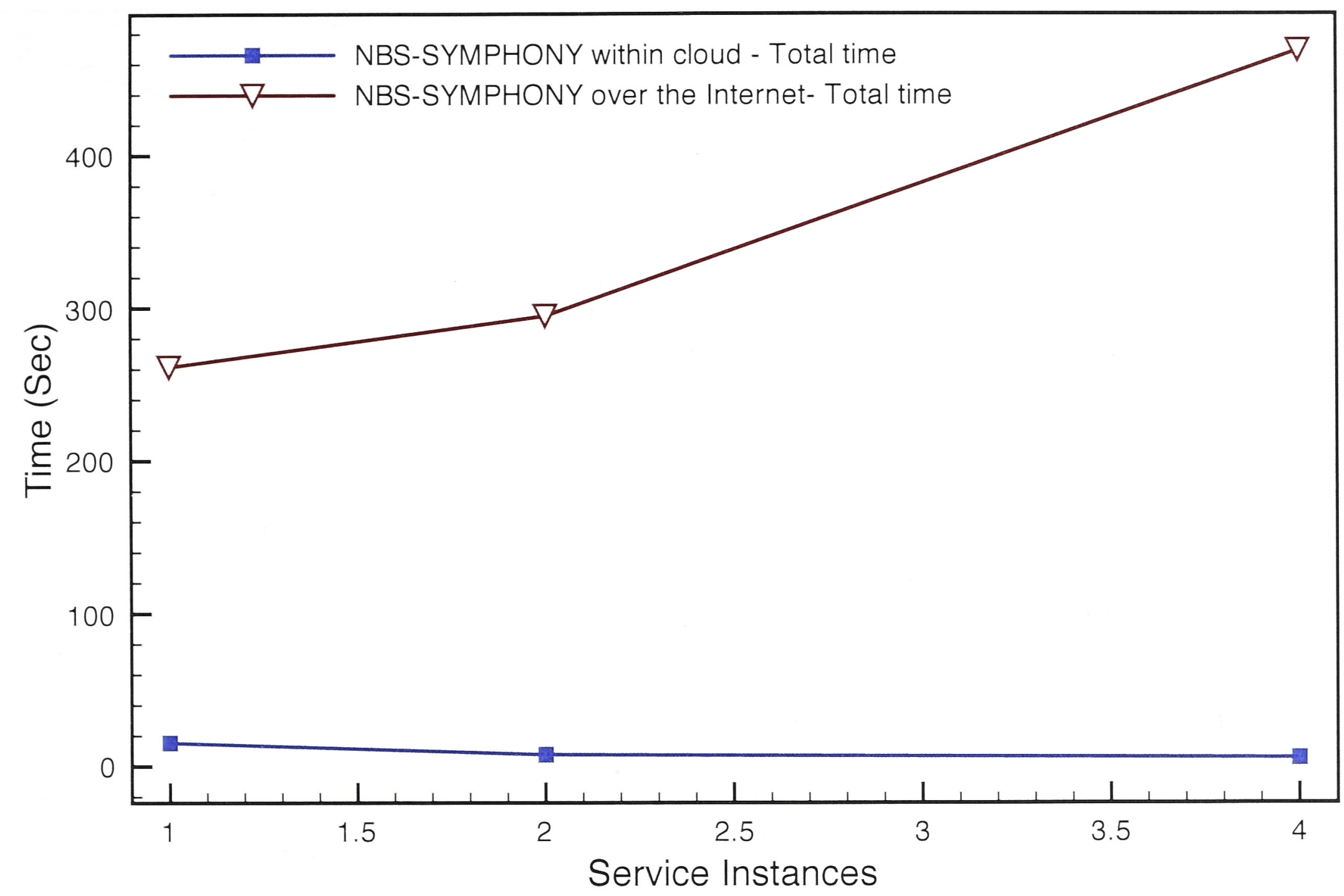

Figure 6.25: NBS Symphony Version within Cloud \& over the Internet

\subsubsection{Performance by Accessing Cloud over the Internet}

Both Symphony and ANU-SOAM versions of NBS were tested in this scenario. The graph shown in Figure 6.24 presents the results of ANU-SOAM NBS over the Internet against its performance within the Rackspace cloud. The computational time increases, but scales as we increase the number of SIs from 1 to 4 . There is a progressive increase in the Common Data loading time. It was noted that, this loading time is almost halved, when separate Rackspace node is used for each SI. But more than one Rackspace node cannot be used to evaluate the performance, as explained before (limitation of OpenMPI).

The results of conventional SOA/Symphony version of NBS over the Internet is presented in graph shown in Figure 6.25. The total compute time jumps to significantly higher levels and we are getting a reverse scaling. A very noisy Internet condition in which the experiments were conducted might have exponentially affected the bad performance of this model (however, similar conditions have been used for ANU-SOAM NBS experiments). This result is in sharp contrast with the results we got when we ran the same version of NBS within cloud (refer to Figure 6.23) or within the homogeneous environment in Vayu cluster (refer to 


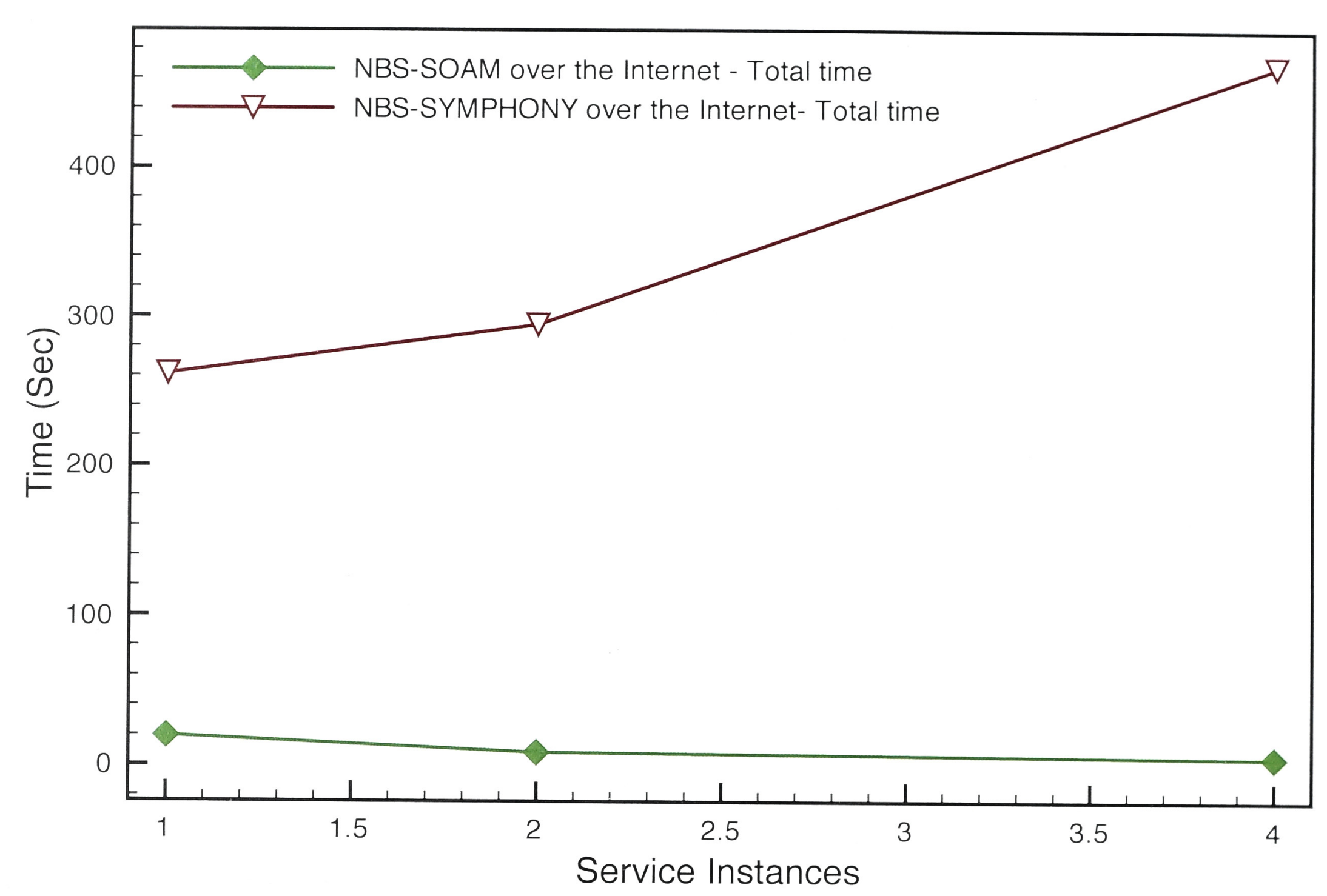

Figure 6.26: NBS SOAM \& Symphony Versions over the Internet

Figure 6.1). It suggests that the conventional SOA/Symphony model is incapable of producing acceptable performance outcomes in this cloud scenarios for kernels like NBS. The large number of communications and data transfer between the client and SIs are adversely affecting its performance. On the other hand, the comparison of both SOA/Symphony and ANU-SOAM versions of NBS over the Internet in Figure 6.26 suggests that the ANU-SOAM programming model is much more equipped to perform well in a cloud environment than the conventional SOA model, if the cloud resources have to be accessed over the Internet by a client. Even though it is not a perfect example of a real time scenario, these results shed light into the advantages of using ANU-SOAM for cloud enabling real time applications over the Internet.

\subsection{Chapter Conclusions}

The experiments on homogeneous conditions show that ANU-SOAM can produce high performance results for medium-grained parallel algorithms. NBS, HTS and CGS show acceptable scaling properties up to 32 nodes on a high performing 
homogeneous network of Vayu cluster at NCI. This is achieved by empowering the application programmer to employ clever algorithms in reducing the communication and data transfer costs with the help of the Data Service extension of ANU-SOAM. It shows comparable or even better performance results to the conventional SOA/Symphony model or even the conventional MPI model of application in some cases. If the individual task granularity slips below optimum level, or in the case of fine-grained parallelism, ANU-SOAM may fail to produce good scaling performance as we have seen in CFS experiments. The inference is that ANU-SOAM is successful in stretching the capabilities of conventional SOA to enable high performance outputs for medium-grained scientific problems, but fine-grained parallel problems may still be beyond the scope of SOA because of the intrinsic limitations of this model. This is consistent with the original assumption of the research work (refer to section 1.3). The load balancing and scheduling efforts of RM module has helped ANU-SOAM to perform better in heterogeneous conditions. Around $20 \%$ performance improvement is achieved by enabling load balancing function in most cases. ANU-SOAM performs exceptionally well compared to its conventional SOA/Symphony counterpart in making use of cloud resources from a remote PC over Internet, when its Data Service extension is used to develop applications that reduce communications between the client and the SIs. In this age of cloud computing this property of ANU-SOAM may open up the possibilities of transforming consumer and mobile devices to supercomputing tools which can respond to real-time needs of high performance applications. 


\section{Chapter 7}

\section{Conclusions \& Future Work}

\subsection{Conclusions}

The aim of this Phd research was to produce a viable and efficient SOA solution to deal with scientific problems that require high performance results. The interdependency of tasks which resulted in finer granularity of tasks was the greatest challenge faced in parallelizing scientific applications using SOA model. There are existing solutions to deal with coarse-grained (or "embarrassingly parallel") scientific applications. So, the scope of this thesis was limited to address the challenges of medium-grained scientific problems, with an assumption that finegrained problems may be too difficult to be dealt with an SOA model.

\subsubsection{High Performance SOA for Scientific Computing}

The research has extended the capability of SOA model to address the challenges in parallelizing medium-grained scientific kernels to produce high performance outcomes. The creative use of MPI in the development of ANU-SOAM has resulted in improving the performance results compared to Platform Symphony (refer to chapter 6). The Data Service extension further enhanced performance of ANU-SOAM applications/kernels by allowing tasks to add, get, put and sync Common Data at client and service instances. The deferred implementation of put functionality allows generations of tasks to modify Common Data within the Data Service without compromising data consistency. Those Data Service functionalities have inspired a paradigm shift in application programming by allowing programmers to transfer most of the application code to the service side and thereby apparently increase the task granularity of otherwise fine grained sci- 
entific parallel problems. The Data Service functionalities were also successful in reducing communications and data transfers between client and service instances.

The efforts to use ANU-SOAM to implement four major and diverse scientific kernels have resulted in extending many functionalities of ANU-SOAM to better suit for scientific computing. The NBS is a compute as well as data transfer intensive kernel. Every data element has to interact with the rest of elements and update it for next generation of tasks, in NBS. The Data Service functions - add, get, put and sync - are designed as a response to these challenges. HTS compelled ANU-SOAM to have a put_b functionality (refer to section 5.2.2), two dimensional Common Data and global indexing of that data (refer to section 4.1.6). CGS problem opened up the possibility of multiple procedures within a task and was instrumental in introducing tasklets functionality (refer to section 5.3.2) and the effective use of non-replicated Common Data. CFS provided challenges of constantly changing boundaries of sub-sets of Common Data on the runtime which resulted in providing varying task number capability to ANUSOAM (refer to section 6.1.2).

ANU-SOAM kernels perform better than its conventional SOA counterparts in most cases. The ANU-SOAM performance is also comparable with that of existing paradigms like MPI under test conditions. The results suggest that medium-grained applications can scale well on ANU-SOAM under homogeneous conditions.

\subsubsection{Efficiency under Heterogeneous Conditions}

On heterogeneous conditions, ANU-SOAM can improve performance of its applications up to $20 \%$ with the help of its load balancing functionalities. This can be achieved without any additional coding efforts for the application programmer. These load balancing capabilities of ANU-SOAM make it heterogeneity-oblivious and different from many conventional HPC tools like MPI that are agnostic about underlying heterogeneous environments (unless additional efforts are taken to address them). ANU-SOAM load balancing technique can produce up to $96 \%$ efficiency of the theoretical expectations in heterogeneous environments under test conditions. 


\subsubsection{Cloud Compatibility}

Even though cloud computing was barely mainstream when this research started, it was assumed that the unique architecture and the Data Service of ANU-SOAM will make it suitable for cloud computing paradigm. This research explored these possibilities and proved that ANU-SOAM programming model can significantly improve performance in accessing cloud IaaS resources over the Internet. ANUSOAM has the potential to function as a PaaS for scientific computing with further refinement.

\subsubsection{Programmability for Scientific Application Develop- ment}

Important features and APIs of Platform Symphony are preserved in ANUSOAM. This decision had helped to retain convenience, simplicity, programmability and other SOA properties of Platform Symphony in ANU-SOAM. It also enables any Symphony application to be easily ported to ANU-SOAM with very little modifications. This portability increases the impact factor of this research, because Platform Computing - the parent company of Platform Symphony - has been recently acquired by IBM to make Symphony a part of IBM cloud computing suite.

\subsection{Future Work}

\subsubsection{Fault Tolerance}

One of the major characteristics of SOA is its capacity to ensure fault-tolerance. In conventional SOA model, it is enabled with the help of hierarchical system of management modules that monitor each other and step in when a crisis arises. In a typical scenario, for example in Platform Symphony, Symphony Session Manager (SSM) monitors the Service Instance Managers (SIM) and The Session Managers are again monitored by a top level Session Director (SD) (refer to Figure 2.4). If any of the SIs fails, it is identified by the SSM, if an SSM fails it is identified by the SD and protocols are initiated to correct, restore or restart the failed unit. If the SD fails, it will be picked up by an SSM and efforts will be made to restart the SD or initiate an appropriate protocol for damage control (refer to section 2.4.2). 
Since ANU-SOAM has retained Platform Symphony architecture, similar fault tolerant mechanisms and protocols can be built in it. SI modules in ANU-SOAM has to be provided with SIMs. The SSMs has to be introduced to monitor those SIMs for every application. The RM module in ANU-SOAM can be empowered with the fault tolerance functionalities of the SD. Implementing partially replicated Common Data, that can be replicated in more than one SIs will be a part of bringing this this additional functionality. Automatic caching of this replicated Common Data will not only enhance the fault tolerance capabilities but also the performance outcomes. Building fault tolerance capabilities to ANU-SOAM is a non-trivial work, but it has to be done to make it an enterprise grade middleware in the long run.

\subsubsection{Dynamic Load balancing}

The load balancing capabilities of the ANU-SOAM make it attractive to deal with heterogeneous conditions. At present, only static load balancing capabilities are enabled in ANU-SOAM. That is, ANU-SOAM assumes a prior knowledge of relative capacities of the compute nodes (in terms of their computing power, network strength, memory capabilities). Dynamic load balancing capability can be added to the RM, if the ANU-SOAM is capable of self-learning the compute environment in which it is functioning. There are various ways in which it can be done. One of those ways is to conduct a pilot test by RM to assess the compute environment and then to update this information as the compute process progress by receiving feedbacks from SIs.

\subsubsection{Support for Simultaneous Multiple Applications}

As an experimental work, ANU-SOAM is capable of running only a single application at a time, at the moment. It can be extended to support simultaneous multiple applications. This can be achieved by introducing SSM modules for each of the applications running on it and an SD to monitor those SSMs. 


\section{Bibliography}

[1] G. E. Mueller, "The Hegel legend of thesis-antithesis-synthesis," Journal of the History of Ideas, vol. 19, no. 3, pp. 411-414, June 1958.

[2] "The modern history of computing," Stanford Encyclopedia of Computing, 2012. [Online]. Available: http://plato.stanford.edu/entries/ computing-history/

[3] W. Hoffman, "Cray's mark remains speed with simplicity," Webpage. [Online]. Available: http://mbbnet.umn.edu/hoff/hoff_sc.html

[4] G. Bell and J. Gray, "What's next in high-performance computing?" Communications of $A C M$, vol. 45, no. 2, pp. 91-95, 2002. [Online]. Available: http://doi.acm.org/10.1145/503124.503129

[5] M. P. Papazoglou, P. Traverso, S. Dustdar, and F. Leymann, "Serviceoriented computing: State of the art and research challenges," Computer, vol. 40, no. 11, pp. 38-45, 2007.

[6] "Grid-enabling and virtualizing mission-critical financial services' applications," Platform Computing, Tech. Rep., August 2006.

[7] X. Chu, K. Nadiminti, C. Jin, S. Venugopal, and R. Buyya, "Aneka: Next-generation enterprise grid platform for e-science and e-business applications," in Proceedings of the Third IEEE International Conference on e-Science and Grid Computing. IEEE Computer Society, December 2007, pp. 151-159. [Online]. Available: www.gridbus.org/ papers/AnekaNextGenGrid2007.pdf

[8] M. P. Papazoglou, P. Traverso, S. Dustdar, and F. Leymann, "Serviceoriented computing: A research roadmap," International Journal of Cooperative Information Systems, vol. 17, no. 2, 2008. 
[9] J. C. Werner. (2009, January) Data and functional gridification for high energy physics. [Online]. Available: www.hep.man.ac.uk/u/jamwer/ Grid2006.doc

[10] A. Khan, T. Adye, C. A. J. Brew, F. Wilson, B. Bense, R. D. Cowles, D. A. Smith, D. Andreotti, C. Bozzi, E. Luppi, P. Veronesi, R. Barlow, M. P. Kelly, J. C. Werner, A. Forti, G. Grosdidier, E. Feltresi, A. Petzold, H. Lacker, and J. E. Sundermann, "Grid applications for high energy physics experiments," in Proceedings of the 6th IEEE/ACM International Workshop on Grid Computing. Washington, DC, USA: IEEE Computer Society, 2005, pp. 274-277.

[11] J. C. Werner, "Grid computing in high energy physics using LCG: the BaBar experience," in Proceedings of UK e-Science All Hands Conference, January 2009.

[12] D. Baker and A. Sali, "Genomics protein structure prediction and structural genomics," Science, vol. 294, no. 93, pp. 93-96, 2001.

[13] B. Segal, L. Robertson, F. Gagliardi, and F. Carminati, "Grid computing: the european data grid project," in Nuclear Science Symposium Conference, vol. 1. Lyon, France: IEEE, 2000.

[14] W. Hoschek, J. J. Martinez, A. Samar, H. Stockinger, and K. Stockinger, "Data management in an international data grid project," in Proceedings of the First IEEE/ACM International Workshop on Grid Computing, vol. 1. Springer-Verlag London, UK, January 2000, pp. 77-90.

[15] A. Chervenak, E. Deelman, C. Kesselman, B. Allcock, I. Foster, V. Nefedova, J. Lee, A. Sim, A. Shoshani, B. Drach, D. Williams, and D. Middleton, "High-performance remote access to climatesimulation data: a challenge problemfor data grid technologies," Parallel Computing, vol. 29, pp. $1335-1356,2003$.

[16] CERN, "Cern - lcg," January 2009. [Online]. Available: http: //lcg.web.cern.ch/LCG/

[17] — "Cern - lhc," January 2009. [Online]. Available: http://lhc.web.cern. ch/lhc/ 
[18] — - "CERN," January 2009. [Online]. Available: http://public.web.cern. ch/Public/Welcome.html

[19] B. Allcock, J. Bester, J. Bresnahan, A. L. Chervenak, I. Foster, C. Kesselman, S. Meder, V. Nefedova, D. Quesnel, and S. Tuecke, "Data management and transfer in high-performance computational grid environments," Parallel Computing, vol. 28, no. 5, pp. 789-771, May 2002.

[20] D. Cameron, J. Casey, L. Guy, P. Kunszt, S. Lemaitre, G. McCance, H. Stockinger, K. Stockinger, G. Andronico, W. Bell, I. Ben-Akiva, D. Bosio, R. Chytracek, A. Domenici, F. Donno, W. Hoschek, E. Laure, L. Lucio, P. Millar, L. Salconi, B. Segal, and M. Silander, "Replica management in the european datagrid project," Journal of Grid Computing, vol. 2, pp. 341-351, April 2005. [Online]. Available: http://www.springerlink.com/content/hn407t1803037565/

[21] G. Aloisioa, V. Breton, M. Mirto, A. Murli, and T. Solomonides, "Special section: Life science grids for biomedicine and bioinformatics," Future Generation Computer Systems, vol. 23, no. 3, pp. 367-370, March 2007.

[22] X. Chu, A. Lonie, P. Harris, S. R. Thomas, and R. Buyya, "A service-oriented grid environment for integration of distributed kidney models and resources," Concurrency and Computation: Practice and Experience, vol. 20, no. 9, pp. 1095-1111, June 2008. [Online]. Available: http://dx.doi.org/10.1002/cpe.1285

[23] C. Boeres and V. E. F. Rebello, "Easygrid: towards a framework for the automatic grid enabling of legacy MPI applications," Concurrency and Computation: Practice and Experience, vol. 16, no. 5, pp. 425 - 432, April 2004.

[24] M. Baertschy and X. Li, "Solution of a three-body problem in quantum mechanics using sparse linear algebra on parallel computers," in Supercomputing '01: Proceedings of the 2001 ACM/IEEE conference on Supercomputing (CDROM). NY, USA: ACM, 2001, pp. 47-78.

[25] M. R. Hestenes and E. Stiefel, "Methods of conjugate gradient for solving linear systems," Journal of Research of the National Bureau of Standards, vol. 49, no. 6, pp. 410-436, December 1952. 
[26] M. Suarjana and K. H. Law, "Successive conjugate gradient methods for structural analysis with multiple load cases," International Journal for $\mathrm{Nu}$ merical Methods in Engineering, vol. 37, no. 24, pp. 4185 - 4203, 2005.

[27] U. Kirsch, M. Kocvara, and J. Zowe, "Accurate reanalysis of structures by a preconditioned conjugate gradient method," International Journal for Numerical Methods in Engineering, vol. 55, no. 2, pp. 233-251, 2002.

[28] F. Chao-jiang and Z. Wu, "Parallel computing for finite element structural analysis using conjugate gradient method based on domain decomposition," Journal of Shanghai University (English Edition), vol. 10, no. 6, pp. 517521, December 2007.

[29] J. Hoeflinger, P. Alavilli, and T. Jackson, "Producing scalable performance with OpenMP: Experiments with two CFD applications," Parallel Computing, vol. 27, no. 582-593, 2001. [Online]. Available: http://citeseerx.ist.psu.edu/viewdoc/summary?doi=10.1.1.9.6856

[30] D. Woollard and N. Medvidovic, "High performance software architectures: A connector oriented approach," in Proceedings of the Institute for Software Research Symposium, Irvine, California, June 2006. [Online]. Available: www.isr.uci.edu/events/GSRS-2006/papers/Woollard.pdf

[31] B. Mohr, F. Bassetti, K. Davis, S. Huttemann, P. Launay, D. C. Marinescu, D. J. Miller, R. L. Vandewart, M. Muller, and A. Prodan, "Parallel highperformance object-oriented scientific computing," in Proceedings of the Workshop on Object-Oriented Technology. London, UK: Springer-Verlag, 1999, pp. 222-239.

[32] S. Miles, E. Deelman, P. Groth, K. Vahi, G. Mehta, and L. Moreau, "Connecting scientific data to scientific experiments with provenance," in Proceedings of the Third IEEE International Conference on eScience and Grid Computing (e-Science 2007). Washington, DC, USA: IEEE Computer Society, 2007, pp. 179-186. [Online]. Available: http://dx.doi.org/10.1109/E-SCIENCE.2007.22

[33] P. H. Worley, "Performance and performance engineering in climate modeling," Online powerpoint material. [Online]. Available: www.sdsc. edu/pmac/workshops/geo2006/pubs/Worley.pdf 
[34] D. Puppin, N. Tonellotto, and D. Laforenza, "How to run scientific applications over web services," in Proceedings of the 2005 International Conference on Parallel Processing Workshops (ICPPW '05), Institute for Information Science \& Technology, Pisa, Italy. IEEE Computer Society, 2005, pp. 29- 33. [Online]. Available: http://dx.doi.org/10.1109/ICPPW. 2005.39

[35] M. Bichier and K. J. Lin, "Service-oriented computing," Computer, vol. 39, no. 3, pp. 99-101, March 2006.

[36] D. Gannon, B. Plale, M. Christie, L. Fang, Y. Huang, S. Jensen, G. Kandaswamy, S. Marru, S. L. Pallickara, S. Shirasuna, Y. Simmhan, A. Slominski, and Y. Sun, "Service oriented architectures for science gateways on grid systems," in Proceedings of Third International Conference on Service Oriented Computing (ICSOC 2005), ser. LNCS, B.benatallah, F.Casati, and Traverso, Eds., ICSOC. Amsterdam, The Netherland: Springer-Verlag, December 2005, pp. 21-32.

[37] M. P. Papazoglou and W.-J. van den Heuvel, "Service oriented architectures: approaches, technologies and research issues," The International Journal on Very Large Data Bases, vol. 16, no. 3, pp. 389-415, July 2007.

[38] M. N. Huhns and M. P. Singh, "Service-oriented computing: Key concepts and principles," IEEE Internet Computing, vol. 9, no. 1, pp. 75-81, January-February 2005.

[39] "Symphony application development guide," Platform Computing Inc, Tech. Rep., 2008.

[40] "Symphony reference," Platform Computing Inc, Tech. Rep., 2009.

[41] I. Foster, "Service-oriented science," Science, vol. 308, no. 5723, pp. $814-$ 817, May 2005.

[42] J. Dongarra and A. Lastovetsky, "An overview of heterogeneous high performance and grid computing," Engineering the Grid: Status and Perspective, February 2006.

[43] A. Sheth, J. Cardoso, J. Miller, and K. Kochut, "QoS for service-oriented middleware," in Proceedings of the 6th World Multiconference on Systemics, Cybernetics and Informatics (SCI02), 2002, pp. 528-534. 
[44] S. Krishnan and K. Bhatia, "SOAs for scientific applications: Experiences and challenges," Future Generations Computer Systems, vol. 25, no. 4, pp. 466-473, April 2009.

[45] S. Gerić, "The potential of service-oriented architectures," in 32nd International Conference on Information Technology Interfaces (ITI). IEEE, June 2010, pp. 471- 476 .

[46] L. Srinivasan and J. Treadwell, "An overview of SOA, web services and grid computing," HP Software Global Business Unit, Tech. Rep., 2005.

[47] J. Zhou, "BLAST Symphony implementation," Platform Computing Internal Document, August 2007, (proprietary and confidential).

[48] I. Foster and C. Kesselman, "Globus: A metacomputing infrastructure toolkit," International Journal of Supercomputer Applications, vol. 11, pp. 115-128, 1997. [Online]. Available: http://citeseerx.ist.psu.edu/viewdoc/ summary?doi=10.1.1.41.6688

[49] I. Foster, C. Kesselman, J. Nick, and S. Tuecke, "The physiology of the grid: An open grid services architecture for distributed systems integration," Open Grid Service Infrastructure Working Group - Global Grid Forum, Tech. Rep., 2002. [Online]. Available: www.globus.org/ alliance/publications/papers/ogsa.pdf

[50] I. Foster, C. Kesselman, J. M. Nick, and S. Tuecke, "Grid services for distributed system integration," Computer, vol. 35, no. 6, pp. 37-46, 2002.

[51] M. Baker, R. Buyya, and D. Laforenza, Grids and Grid technologies for wide-area distributed computing. England: John Wiley \& Sons, Ltd, 2002. [Online]. Available: www.gridbus.org/papers/gridtech.pdf

[52] M. P. Thomas, J. Burruss, L. Cinquini, G. Fox, D. Gannon, L. Gilbert, G. von Laszewski, K. Jackson, D. Middleton, R. Moore, M. Pierce, B. Plale, A. Rajasekar, R. Regno, E. Roberts, D. Schissel, A. Seth, and W. Schroeder, "Grid portal architectures for scientific applications," Journal of Physics, vol. Series 16, pp. 596 - 600, 2005. [Online]. Available: http://www.iop.org/EJ/abstract/1742-6596/16/1/083

[53] A. A. Tantar, N. Melab, E. G. Talbia, B. Parent, and D. Horvath, "A parallel hybrid genetic algorithm for protein structure prediction on the 
computational grid," Future Generation Computer Systems, vol. 23, no. 3, pp. 398-409, March 2007.

[54] P. Asadzadeh, R. Buyya, C. L. Kei, D. Nayar, and S. Venugopal, "Global grids and software toolkits: A study of four grid middleware technologies," in High Performance Computing: Paradigm and Infrastructure, L. Yang and M. Guo, Eds. Wiley Press, USA, 2005. [Online]. Available: www.gridbus.org/papers/gmchapter.pdf

[55] R. Buyya and S. Venugopal, "A gentle introduction to grid computing and technologies," Computer Society of India (CSI) Communicaitons, vol. 29, no. 1, pp. 9-19, July 2005. [Online]. Available: www.gridbus.org/papers/ GridIntro-CSI2005.pdf

[56] D. W. Erwin and D. F. Snelling, "Unicore: A grid computing environment," in Euro-Par 2001 Parallel Processing, ser. Lecture Notes in Computer Science, R. Sakellariou, J. Gurd, L. Freeman, and J. Keane, Eds. Springer-Verlag, January 2001, vol. 2150, pp. 825-834.

[57] D. Abramson, R. Buyya, and J. Giddy, "A computational economy for grid computing and its implementation in the Nimrod-G resource broker," Future Generation Computer Systems, vol. 18, no. 8, pp. 1061-1074, 2002.

[58] R. Buyya, D. Abramson, and J. Giddy, "A case for economy grid architecture for service-oriented grid computing," in Proceedings of the 10th Heterogeneous Computing Workshop in conjunction with IPDPS '01, vol. 2, IPDPS. Washington, DC, USA: IEEE Computer Society, 2001, p. 20083.1.

[59] J. Broberg, S. Venugopal, and R. Buyya, "Market-oriented grids and utility computing: The state-of-the-art and future directions," Journal of Grid Computing, vol. 6, no. 3, pp. 255-276, September 2008.

[60] "Common component architecture forum," 2012. [Online]. Available: http://www.cca-forum.org

[61] D. E. Bernholdt, B. A. Allan, R. Armstrong, F. Bertrand, K. Chiu, T. L. Dahlgren, K. Damevski, W. R. Elwasif, T. G. W. Epperly, M. Govindaraju, D. S. Katz, J. A. Kohl, M. Krishnan, G. Kumfert, J. W. Larson, S. Lefantzi, M. J. Lewis, A. D. Malony, L. C. McInnes, J. Nieplocha, B. Norris, S. G. Parker, J. Ray, S. Shende, T. L. Windus, 
and S. Zhou, "A component architecture for high-performance scientific computing," International Journal of High Performance Computing Applications, vol. 20, no. 2, pp. 163-202, 2006. [Online]. Available: http://hpc.sagepub.com/cgi/content/abstract/20/2/163

[62] R. Armstrong, D. Gannon, A. Geist, K. Keahey, S. Kohn, L. McInnes, S. Parker, and B. Smolinski, "Toward a common component architecture for high-performance scientific computing," in Proceedings of the 8th IEEE International Symposium on High Performance Distributed Computing (HPDC '99). IEEE Computer Society, 1999, pp. 115-124.

[63] L. Baresi, R. Heckel, S. Thone, and D. Varro, "Modeling and validation of service oriented architectures: Application vs style," in Proceedings of the 9th European software engineering conference held jointly with 11th ACM SIGSOFT international symposium on Foundations of software engineering. New York, NY, USA: ACM, 2006, pp. 68 - 77. [Online]. Available: http://doi.acm.org/10.1145/940071.940082

[64] L. Youseff, M. Butrico, and D. D. Silva, "Toward a unified ontology of cloud computing," in Proceedings of the Grid Computing Environments Workshop. Santa Barbara, CA, USA: IEEE, 2008, pp. 1 - 10.

[65] I. Foster, Y. Zhao, I. Raicu, and S. Lu, "Cloud computing and grid computing 360-degree compared," in Proceedings of the IEEE Grid Computing Environments Workshop, no. 1-10. IEEE, November 2008.

[66] M. Armbrust, A. Fox, R. Griffith, A. D. Joseph, R. H. Katz, A. Konwinski, G. L. D. A. Patterson, A. R. I. Stoica, and M. Zaharia, "Above the clouds: A Berkeley view of cloud computing," University of California at Berkeley, Technical Report UCB/EECS-2009-28, February 2009.

[67] R. G. A. D. J. MiChAEL Armburst, ARMAndO Fox, R. Katz, A. Konwinski, G. Lee, D. Patterson, A. Rabkin, I. Stoica, and M. Zaharia, "A view of cloud computing," Communications of ACM, vol. 53, no. 4, pp. 50-59, April 2010.

[68] P. Sempolinski and D. Thain, "A comparison and critique of Eucalyptus, OpenNebula and Nimbus," in In Proceedings of International Conference on Cloud Computing Technology and Science. IEEE, December 2010, pp. $417-426$. 
[69] "Reimagining IT: Insights from the 2011 gartner cio agenda," Report, Gartner Inc, Tech. Rep., 2011.

[70] R. Buyya, C. S. Yeo, S. Venugopal, J. Broberg, and I. Brandic, "Cloud computing and emerging IT platforms: Vision, hype, and reality for delivering computing as the 5th utility," Future Generation Computer Systems, vol. 25, no. 6, pp. 599-616, June 2009.

[71] P. Mell and T. Grance, "The NIST definition of cloud computing," National Institute of Standards and Technology, Special Publication 800-145, September 2011.

[72] R. Buyya, S. Pandey, and C. Vecchiola, "Cloudbus toolkit for marketoriented cloud computing," in Proceedings of the 1st International Conference on Cloud Computing, ser. CloudCom '09, vol. 5931/2009, LNCS. Berlin, Heidelberg: Springer-Verlag, 2009, pp. 24-44.

[73] C. Vecchiola, X. Chu, and R. Buyya, "Aneka: A software platform for .netbased cloud computing," in High Performance Computing Workshop, ser. Advances in Parallel Computing, W. Gentzsch, L. Grandinetti, and G. R. Joubert, Eds., vol. 18. IOS Press, 2009, pp. 267-295.

[74] K. Keahey, T. Freeman, R. Figueiredo, J. Fortes, and M. Tsugawa, "Science clouds: Early experiences in cloud computing for scientific application," Nimbus Cloud Project, Tech. Rep., 2008. [Online]. Available: http://nimbusproject.org/files/Science-Clouds-CCA08.pdf

[75] "The private cloud revolution: Fully utilizing your existing compute, storage and networking assets," Eucalyptus, Tech. Rep., 2011. [Online]. Available: http://www.eucalyptus.com/resources/whitepapers

[76] L. Wang, G. von Laszewski, A. Younge, X. He, M. Kunze, J. Tao, and C. Fu, "Cloud computing: a perspective study," New Generation Computing, vol. 28, no. 2, pp. 137-146, December 2008.

[77] M. A. Vouk, "Cloud computing - issues, research and implementations," Journal of Computing and Information Technology, vol. 16, no. 4, pp. 235 $246,2008$.

[78] T. W. Wlodarczyk and C. Rong, "An initial survey on integration and application of cloud computing to high performance computing," in Third 
IEEE International Conference on Cloud Computing Technology and Science. IEEE, 2011.

[79] E. Roloff, M. Diener, A. Carissimi, and P. O. A. Navaux, "High performance computing in the cloud: Deployment, performance and cost efficiency," in 4th International Conference on Cloud Computing Technology and Science. IEEE, December 2012, pp. 371-378.

[80] A. Iosup, S. Ostermann, M. N. Yigitbasi, R. Prodan, T. Fahringer, and D. H. Epema, "Performance analysis of cloud computing services for manytasks scientific computing," IEEE Transactions on Parallel and Distributed Systems, vol. 22, no. 6, pp. 931-946, June 2011.

[81] P. Jakovits, S. N. Srirama, and I. Kromonov, "Stratus: A distributed computing framework for scientific simulations on the cloud," in 14th International Conference on High Performance Computing and Communication and Ith International Conference on Embedded Software and Systems (HPCC-ICESS). IEEE, June 2012, pp. 1053 - 1059.

[82] M. Villamizar, H. Castro, and D. Mendez, "e-clouds: A saas marketplace for scientific computing," in Fifth International Conference onUtility and Cloud Computing (UCC). IEEE, 2012, pp. 13-20.

[83] Y. Zhao, Y. Zhang, W. Tian, R. Xue, and C. Lin, "Designing and deploying a scientific computing cloud platform," in 13th International Conference on Grid Computing (GRID). IEEE/ACM, September 2012.

[84] S. Zhou, "Performance studies of dynamic load balancing in distributed systems," Ph.D. dissertation, UC Berkley, 1987.

[85] "Platform computing - history," January 2012. [Online]. Available: www.platform.com/company/history

[86] G. Sissons, "A developer's guide to building high performance serviceoriented applications," Platform Computing Inc, Tech. Rep., March 2010.

[87] IBM, "IBM acquires platform computing," February 2012. [Online]. Available: http://www-03.ibm.com/systems/deepcomputing/platform.html

[88] G. Sissons and O. Parmar, "High performance SOA with Platform Symphony 5.1," Platform Computing Inc, Tech. Rep., April 2011. 
[89] J. Mulerikkal and P. Strazdins, "Service oriented approach to high performance scientific computing," in Proceedings of the Seventh IEEE International Symposium on Cluster Computing and the Grid (CCGRID '10), M. Parashar and R. Buyya, Eds. CPS, 2010, pp. 820-825.

[90] W. Gropp, E. Lusk, and A. Skjellum, Using MPI: Portable Parallel Programming with the Message-Passing Interface. MIT Press, 1999.

[91] A. C. Sena, A. P. Nascimento, C. Boeres, and V. E. F. Rebello, "Easygrid enabling of iterative tightly-coupled parallel mpi applications," in International Symposium on Parallel and Distributed Processing with Applications (ISPA '08). IEEE Computer Society, December 2008, pp. 199-206.

[92] I. T. Foster, "Globus toolkit version 4: Software for service-oriented systems." in Proceedings of the international conference on Network and Parallel Computing, ser. LNCS, H. Jin, D. A. Reed, and W. Jiang, Eds., vol. 3779. Berlin, Heidelberg: Springer-Verlag, 2005, pp. 2-13. [Online]. Available: www.globus.org/alliance/events/sc05/GT4.pdf

[93] C. Boeres, A. A. Fonseca, H. A. Mendes, L. T. Menezes, N. T. Moura, J. A. Silvaand, B. A. Vianna, and V. E. F. Rebello, "An Easygrid portal for scheduling system-aware applications on computational grids," Concurrency and Computation: Practice and Experience, vol. 18, no. 6, pp. $553-$ 566, May 2006.

[94] C. Boeres, V. E. F. Rebello, and A. P. Nascimento, "Dynamic selfscheduling for parallel applications with task dependencies," in Proceedings of the 6th international workshop on Middleware for grid computing, no. 1. NY, USA: ACM, 2008, pp. 1-6.

[95] A. P. Nascimento, C. Boeres, and V. E. F. Rebello, "Distributed and dynamic self-scheduling of parallel mpi grid applications," in $M G C$ '08: Proceedings of the 6th international workshop on Middleware for grid computing. New York, NY, USA: ACM, 2008, pp. 1-6.

[96] A. Nascimento, A. Sena, J. da Silva, D. Vianna, C. Boeres, and V. Rebello, "Managing the execution of large scale MPI applications on computational grids," in International Symposium on Computer Architecture and High Performance Computing, 2005, pp. 69-76. 
[97] "HeteroMPI," October 2010. [Online]. Available: http://hcl.ucd.ie/ project/HeteroMPI

[98] A. Lastovetsky and R. Reddy, "HeteroMPI: Towards a message-passing library for heterogeneous networks of computers," Journal of Parallel and Distributed Computing, vol. 66, no. 2, pp. 197-220, February 2006.

[99] A. Lastovetsky and V. Rychkov, "Optimization of collective communications in heteroMPI," in Recent Advances in Parallel Virtual Machine and Message Passing Interface. Springler, 2007, vol. 4757/2007, pp. 135-143.

[100] T. Desell, K. E. Maghraoui, and C. A. Varela, "Malleable applications for scalable high performance computing," Cluster Computing, vol. 10, no. 3, pp. 323-337, 2007.

[101] K. E. Maghraoui, T. J. Desell, B. K. Szymanski, and C. A. Varela, "Dynamic malleability in iterative MPI applications," in International Symposium on Cluster Computing and the Grid (CCGRID 2007). IEEE, May 2007, pp. 591-598. [Online]. Available: http://ieeexplore.ieee.org/ stamp/stamp.jsp?arnumber $=4215427 \&$ isnumber $=4215349$

[102] "About openMP ARB and openMP.org," openMP.org, Tech. Rep., January 2009. [Online]. Available: http://openmp.org/wp/about-openmp/

[103] C. Liao, O. Hernandez, B. Chapman, W. Chen, and W. Zheng, "OpenUH: an optimizing, portable OpenMP compiler," Concurrency and Computation: Practice and Experience, vol. 19, no. 18, pp. 2317-2332, December 2007. [Online]. Available: http://dx.doi.org/10.1002/cpe.v19:18

[104] "OpenMP application program interface - ver 2.5," OpenMP.org, Tech. Rep., 2008. [Online]. Available: www.openmp.org/mp-documents/spec30. pdf

[105] "How should I choose between using OpenMP or MPI for parallel computing?" March 2012. [Online]. Available: http://www.hpsc.csiro.au/ userguides/faq/parallel_choice.php

[106] J. Cai, P. E. Strazdins, , and A. P. Rendell, "Region-based prefetch techniques for software distributed shared memory systems," in Proceedings of the10th International Conference on Cluster, Cloud and Grid Computing (CCGrid), 2010. IEEE/ACM, 2010, pp. 113 - 122. 
[107] J. Cai, A. P. Rendell, P. E. Strazdins, and H. J. Wong, "Performance models for cluster-enabled OpenMP implementations," in Proceedings of the 13th Asia-Pacific Computer Systems Architecture Conference. IEEE, 2008, pp. $1-8$.

[108] "ScaleMP." [Online]. Available: http://www.scalemp.com/performance

[109] L. Adhianto and B. Chapman, "Performance modeling of communication and computation in hybrid MPI and OpenMP applications," in Proceedings of the 12th International Conference on Parallel and Distributed Systems (ICPADS '06), vol. 2. Washington, DC, USA: IEEE Computer Society, 2006, pp. 3-8.

[110] A. Smyk and M. Tudruj, "OpenMP/MPI Programming in a multi-cluster system based on shared memory/message passing communication," Advanced Environments, Tools, and Applications for Cluster Computing, vol. 2326/2002, pp. 157-160, 2002.

[111] "Global arrays," Website, 2009. [Online]. Available: http://acts.nersc.gov/ ga/index.html

[112] J. Nieplocha, R. J. Harrison, and R. J. Littlefield, "Global arrays: a portable shared-memory programming model for distributed memory computers," in Proceedings of the ACM/IEEE conference on Supercomputing (Supercomputing '94). New York, NY, USA: ACM, 1994, pp. 340-349. [Online]. Available: http://doi.acm.org/10.1145/602770.602833

[113] — - "The global array programming model for high performance scientific computing," Society for Industrial and Applied Mathematics Journal on Computing, vol. 28, 1995.

[114] "The GA toolkit," Webpage, 2008. [Online]. Available: http://www.emsl. pnl.gov/docs/global/

[115] G. Antoniu, E. Caron, F. Desprez, A. Fèvre, and M. Jan, "Towards a transparent data access model for the GridRPC paradigm," in Proceedings of the14th International Conference on High Performance Computing. Berlin, Heidelberg: Springer-Verlag, 2007, pp. 269-284. 
[116] J. Mulerikkal and P. Strazdins, "Efficient data service design for a soa approach to scientific computing," in Proceedings of the Tenth IASTED International Conference on Parallel and Distributed Computing and Networks, IASTED. Innsbruck: ACTA Press, February 2011, pp. 46-53.

[117] J. Nieplocha, B. Palmer, V. Tipparaju, M. Krishnan, H. Trease, and E. Apra, "Advances, applications and performance of the global arrays shared memory programming toolkit," International Journal of High Performance Computing Applications, vol. 20, no. 203, 2006.

[118] A. Chervenak, I. Foste, C. Kesselman, and C. Salisbury, "The data grid: Towards an architecture for the distributed management and analysis of large scientific datasets," Journal of Network and Computer Applications, vol. 23, pp. 187-200, 2000.

[119] S. Ghemawat, H. Gobioff, and S. T. Leung, "The Google file system," in Proceedings of the nineteenth ACM symposium on Operating systems principles, vol. 37, no. 5. ACM, 2003, pp. 29-43.

[120] D. Borthakur, "HDFS architecture," Hadoop 0.19 Documentation, Apache Hadoop, Tech. Rep., 2008.

[121] "What hadoop is not," May 2010. [Online]. Available: http://wiki.apache. org/hadoop/HadoopIsNot

[122] D. C. Schmidt, "The adaptive communication environment: An objectoriented network programming toolkit for developing communication software," in Sun User Group conference. St. Louis, MO 63130: Department of Computer Science, Washington University, 1993. [Online]. Available: www.cs.wustl.edu/ schmidt/PDF/SUG-94.pdf

[123] J. Pješivac-Grbović, T. Angskun, G. Bosilca, G. Fagg, E. Gabriel, and J. Dongarra, "Performance analysis of MPI collective operations," Cluster Computing, vol. 10, pp. 127-143, 2007. [Online]. Available: http://dx.doi.org/10.1007/s10586-007-0012-0

[124] L. Valiant, "Why BSP computers?" in Proceedings of Seventh International Parallel Processing Symposium, Newport, CA, USA, 1993, pp. 2-5.

[125] "Application development guide - Platform Symphony version 4.0," Platform Computing, Tech. Rep., January 2008. 
[126] Platform Symphony Knowledge Center, Platform Computing, February 2012. [Online]. Available: http://my.platform.com/docs/symphonyde/4.1/

[127] R. Spurze, "Direct N-Body simulations," Journal of Computational and Applied Mathematics, vol. 109, no. 1-2, Sept 1999.

[128] D. G. Saari, "Collisions, rings and other newtonian N-Body problems," in The Dynamical Behavior of the Newtonian N Body Problem, ser. NSFCBMS Regional Research Conference, no. 104, Conference Board of the Mathematical Sciences. Providence, Rhode Island, USA: American Mathematical Society, June 2002.

[129] B. Barney, D. Turner, G. L. Gusciora, and C. B. Connor, "Heat2D example - parallelized C version," October 2010. [Online]. Available: www.cas.usf.edu/ cconnor/parallel/2dheat/

[130] T. Saad, "Parallel computing with MPI - part iv: Programming the 2D heat equation in C using Visual Studio." [Online]. Available: http://pleasemakeanote.blogspot.com.au/2008/06/ parallel-computing-with-mpi-part-iv.html

[131] J. Mulerikkal and P. Strazdins, "An SOA approach to high performance scientific computing - early experiences," in Proceedings of International Conference on High Performance Computing 2010, Goa, India, December 2010, pp. 1-10.

[132] H. S. Kim and S. B. Baden, "A study on the performance of choleskyfactorization using MPI," Archieves of University of California San Diego, 2005.

[133] PlanetMath.org. Conjugate gradient algorithm. [Online]. Available: planetmath.org/encyclopedia/MethodOfConjugateGradients.html

[134] F. Domes and A. Neumair, "Directed choleksy factrizations and applications," ePrints for the Optimization Community, University of Vienna, Nordbergstrasse 15, A-1090 Vienna, Austria, 2008. [Online]. Available: http://www.optimization-online.org/DB_HTML/2008/02/1914.html

[135] N. Mateev, V. Menon, K. Pingali, A. Bode, T. Ludwig, W. Karl, and R. Wismüller, Left-Looking to Right-Looking and Vice Versa: An Application of Fractal Symbolic Analysis to Linear Algebra Code 
Restructuring. Springer Berlin / Heidelberg, 2000, vol. 1900, pp. 379-388. [Online]. Available: http://dx.doi.org/10.1007/3-540-44520-X_49

[136] "Sun constellation cluster, vayu: System details," May 2012. [Online]. Available: http://nf.nci.org.au/facilities/vayu/hardware.php

[137] R. Thakur, R. Rabenseifner, and W. Gropp, "Optimization of collective communication operations in MPICH," International Journal of High Performance Computing Applications, pp. 49-66, 2005.

[138] V. Turchenko, L. Grandinetti, G. Bosilca, and J. J. Dongarra, "Improvement of parallelization efficiency of batch pattern BP training algorithm using Open MPI," Procedia Computer Science, vol. 1, no. 1, pp. 525 - 533, 2010. [Online]. Available: http://www.sciencedirect.com/ science/article/pii/S1877050910000578

[139] "Integrated performance monitoring," July 2012. [Online]. Available: http://ipm-hpc.sourceforge.net/

[140] Amazon Elastic Compute Cloud: Public and Private Addresses, 2012 July. [Online]. Available: http://docs.amazonwebservices.com/AWSEC2/latest/ UserGuide/using-instance-addressing.html 


\section{Appendix A}

\section{API}

Important ANU-SOAM Application Programming Interfaces (APIs) are given below. ANU-SOAM implements most of the important Platform Symphony APIs and the corresponding explanations are reproduced from the Platform Symphony documentation given in [126] with necessary modifications. ANU-SOAM provides additional APIs related to the Data Service and RM functionalities.

\section{A.1 Client API}

\section{A.1.1 SoamFactory Class Reference}

void initialize()

Initializes the API. The initialize(...) method should be called before any other soam API method is called. This method is mandatory which means all other API calls will fail if this method is omitted. This should be called only once, since uninitialize(...) can only be called once.

\section{void uninitialize()}

Un-initializes the API. The uninitialize() method should be called at the end of all API calls. It should be called only once.

\section{A.1.2 SessionCreationAttributes Class Reference void setServiceCmd(char* serviceName)}

Sets the name of the service. 


\section{setNoOfChildren(int noOfChildren)}

Sets the number of service instances required.

setRMPolicy (char* rm-policy)

Sets the resource manager policy as "random", "round-robin" or "weightedround-robin". When "weighted-round-robin" policy is used, the relative weights of services instances are read from file "policy.soam" in the same folder.

\section{void setHostInfo()}

Sets a custom path to find service instances, which is read from file "profile.soam" in the same folder.

\section{void setDefaultComm()}

Sets default values for attributes, serviceName= "service", noOfChildren=2, rmpolicy = "round-robin" and service path $=$ "./Service".

\section{A.1.3 ConnectionPtr Class Reference}

SessionPtr* createSession (SessionCreationAttributes \&attributes)

Creates a session on a connection with the given attributes.

\section{A.1.4 SessionPtr Class Reference}

void addCommonData(char* name, dataType* inputBuf, int numberOfElements)

Adds common data (integer or double array) to data service list and sets the same at all SIs.

void getCommonData(char* name, dataType* inputBuf, int numberOfElements)

Gets common data (integer or double array) from common data list. 
void addCommonData(char* name, dataType** inputBuf, int ndim, int $^{*}$ dims)

Adds multi-dimentional (ndim ! >2) common data (integer or double array) to common data list and sets the same at all SIs.

void getCommonData(char* name, dataType** outputBuf, int ndim, int $^{*}$ dims)

Gets multi-dimentional (ndim ! >2) common data (integer or double array) from common data list.

void addCommonData(char* name, dataType* inputBuf, int numberOfElements, int taskTag)

Adds partial common data (integer or double array) to data service list and sets the same at corresponding SI.

void getCommonData(char* name, dataType* inputBuf, int numberOfElements, int taskTag)

Gets partial common data (integer or double array) from common data list from corresponding SI.

void addCommonData(char* name, dataType** inputBuf, int ndim, int* dims, int taskTag)

Adds multi-dimentional (ndim ! >2) common data (integer or double array) to common data list and sets at corresponding SI.

void getCommonData(char* name, dataType** outputBuf, int ndim, int* dims, int taskTag)

Gets multi-dimentional (ndim!>2) common data (integer or double array) from common data list from the corresponding SI.

void syncCommonData(char* name)

Send command to all SIs to synchronize common data uniquely identified by its name and to update it back to client. 
void iSyncCommonData(char* name)

Send command to all SIs to synchronize common data uniquely identified by its name among themselves alone.

void iGetCommonData(char* name, dataType* outputBuf, int numberOfElements)

Send command to an SI to update a common data uniquely identified by its name back to its client common data list and gets it (integer or double array) from the list.

\title{
A.1.5 TaskSubmissionAttributes Class Reference \\ void setTaskInput(Message *taskInput)
}

Sets the input message for the task.

void setTaskTag(int taskTag)

Sets the task tag.

void setTaskletTag(int taskletTag)

Sets the tasklet tag.

void setTaskInput(Message *taskInput)

Sets the input message for the task.

\author{
A.1.6 TaskInputHandlePtr Class Reference \\ int $\operatorname{get} \operatorname{Id}()$ \\ Get the ID of a Task returned by SOAM.
}

\section{A.1.7 TaskOutputHandlePtr Class Reference int $\operatorname{get} \operatorname{Id}()$}

Gets the task ID of a TaskOutputHandle returned by SOAM. 
bool isSuccessful()

Check if a message has been successfully processed by SOAM.

void populateTaskOutput (Message *outputMessage)

Retrieves the output associated with the task.

\section{A.1.8 EnumItemsPtr Class Reference bool getNext(TaskOutputHandlePtr \&outputHandlePtr)}

Returns the next item in the enumeration. If the enumeration's current position is at the end, false will be returned and the taskOutputHandle will be invalid, otherwise true will be returned and the taskOutputHandle will be valid.

int getCount ()

Retrieve a count of the number of items in the enumeration.

\section{A.2 Service API}

\section{A.2.1 ServiceContainer Class Reference void onSessionEnter(SessionContextPtr \&sessionContext)}

The middleware triggers the invocation of this handler to bind the service Instance to its owning session and common data can be accessed here. The application developer must put any session-specific initialization into this handler. Default implementation does nothing.

\section{void onSessionLeave()}

The middleware triggers the invocation of this handler to unbind the service Instance from its owning Session. This handler should do any cleanup for any resources that were used in the onSessionEnter() method. Default implementation does nothing. 


\section{void onInvoke(TaskContextPtr \&taskContext)}

The middleware triggers the invocation of this handler every time a task input is sent to the service to be processed. The actual service logic should be implemented in this method. Common data can be accessed here. This is the only method that is mandatory for the application developer to implement.

\section{A.2.2 SessionContextPtr Class Reference \\ void addCommonData(char* name, dataType* inputBuf, int numberO- fElements)}

Adds common data (integer or double array) to common data list at that particular SI, where this function is called.

void getCommonData(char* name, dataType* inputBuf, int numberOfElements)

Gets common data (integer or double array) from common data list at SI.

void putCommonData(char* name, dataType* startPosition, int numberOfElements, int* inputBuf)

Puts data type (integer of double) array of length "numberOfElements" to common data uniquely identified by its name, beginning from "startPositon". This is a deferred put. The update is stored in a data service list with its meta data and will be updated to common data list and synchronized with other SIs only after a "sync" call from client.

void addCommonData(char* name, dataType* inputBuf, int numberOfElements, int taskTag)

Adds common data (integer or double array) to non-replicated common data list at that particular SI, where this function is called.

void getCommonData(char* name, dataType* inputBuf, int numberOfElements, int taskTag)

Gets common data (integer or double array) from non-replicated common data list at SI. 
void addCommonData(char* name, dataType* inputBuf, int numberOfElements, int taskTag)

Adds partial common data (integer or double array) to data service list and sets the same at corresponding SI.

void getCommonData(char* name, dataType* inputBuf, int numberOfElements, int taskTag)

Gets partial common data (integer or double array) from common data list from corresponding SI.

void addCommonData(char* name, dataType** inputBuf, int ndim, int* dims, int taskTag)

Adds multi-dimentional (ndim ! >2) common data (integer or double array) to common data list and sets at corresponding SI.

void getCommonData(char* name, dataType** outputBuf, int ndim, int* dims, int taskTag)

Gets multi-dimentional (ndim ! >2) common data. (integer or double array) from common data list from the corresponding SI.

void putCommonData(char* name, dataType* startPosition, int numberOfElements, int* inputBuf, int taskTag)

Puts data type (integer of double) array of length "numberOfElements" to nonreplicated common data uniquely identified by its name, beginning from "startPositon". This is not a deferred put rather updates non-replicated common data list immediately.

void addCommonData(char* name, dataType** inputBuf, int ndim, int* dims)

Adds multi-dimentional (ndim! 2) common data (integer or double array) to common data list at that particular SI, where this function is called. 
void getCommonData(char* name, dataType** outputBuf, int ndim, int* dims)

Gets multi-dimentional (ndim! $>2$ ) common data (integer or double array) from common data list at SI.

void getCommonData(char* name, dataType** outputBuf, int ndim, int* dims, int* lo, int* hi)

Gets multi-dimentional (ndim! >2) common data (integer or double array) between higher indices (represented by array 'hi') to lower indices (represented by array 'hi') where lowest indeces starts from $(0,0)$, from common data list at SI.

void putCommonData(char* name, dataType** startPosition, int ndim, int* dims, int* lo, int* hi)

Puts multi-dimentional (ndim ! >2) data type array (integer of double) between higher indices (represented by array ' $h i$ ') to lower indices (represented by array 'hi') where lowest indeces starts from $(0,0)$, common data. The update is stored in a data service list with its meta data and will be updated to common data list and synchronized with other SIs only after a "sync" call from client.

void putCommonData_b(char* name, dataType** startPosition, int ndim, int* dims, int* lo, int* hi)

Puts the inner boundary elements of the multi-dimentional (ndim ! >2) data type array (integer of double) between higher indices (represented by array 'hi') to lower indices (represented by array ' $h i$ ') where lowest indeces starts from $(0,0)$, common data. The update is stored in a data service list with its meta data and will be updated to common data list. Only the inner boundary elements will be synchronized with other SIs after a "sync" call from client.

void putCommonData_i(char* name, dataType** startPosition, int ndim, int* dims, int* lo, int* hi)

Updates multi-dimentional (ndim ! >2) data type array (integer of double) between higher indices (represented by array 'hi') to lower indices (represented by array 'hi') where lowest indeces starts from $(0,0)$, to common data list of that specific SI instantaneously. This is not a deferred action rather updates common data list immediately. 


\section{A.2.3 Task Class Reference \\ int getTaskId ()}

Gets the ID of a Task returned by SOAM.

void populateTaskInput(Message \&inputMessage)

Populates a message objecte with the input of a Task.

void setTaskOutput(Message \&outputMessage)

Sets an output message of a Task.

\section{A.3 Message API}

\section{A.3.1 Message Class Reference}

void onSerialize(OutputStreamPtr \&stream)

This method will be implemented by the SOAM developer. It will serialize its content to the stream object provided.

void onDeserialize(InputStreamPtr \&stream)

This method will be implemented by the SOAM developer. It will de-serialize the contents of the stream provided and populate the object.

\section{A.3.2 InputStreamPtr Class Reference}

void $\operatorname{read}($ int $\& x)$

This method will read an integer from the input stream.

void read(double $\& \mathbf{x})$

This method will read a double from the input stream.

void $\operatorname{read}(\operatorname{char} \& \mathrm{x})$

This method will read an char from the input stream. 
void $\operatorname{read}\left(\operatorname{void} \operatorname{readBytes}\left(\right.\right.$ unsigned $\operatorname{char}^{*} \mathrm{x}$, int \&len))

This method will read a byte array of length 'len' from the input stream.

\section{A.3.3 OutputStreamPtr Class Reference}

void write(int $\mathrm{x}$ )

This method will write an integer to the output stream.

void write(double $\mathrm{x}$ )

This method will write a double to the output stream.

void write $(\operatorname{char} \mathrm{x})$

This method will write a char to the output stream.

void $\operatorname{read}(\operatorname{void}$ writeBytes(unsigned char* $\mathrm{x}$, int len))

This method will write a byte array of length 'len' to the output stream. 


\section{Appendix B}

\section{Code Snippets}

\section{B.1 Client Module}

Listing B.1: Initialize and Uninitialize ANU-SOAM

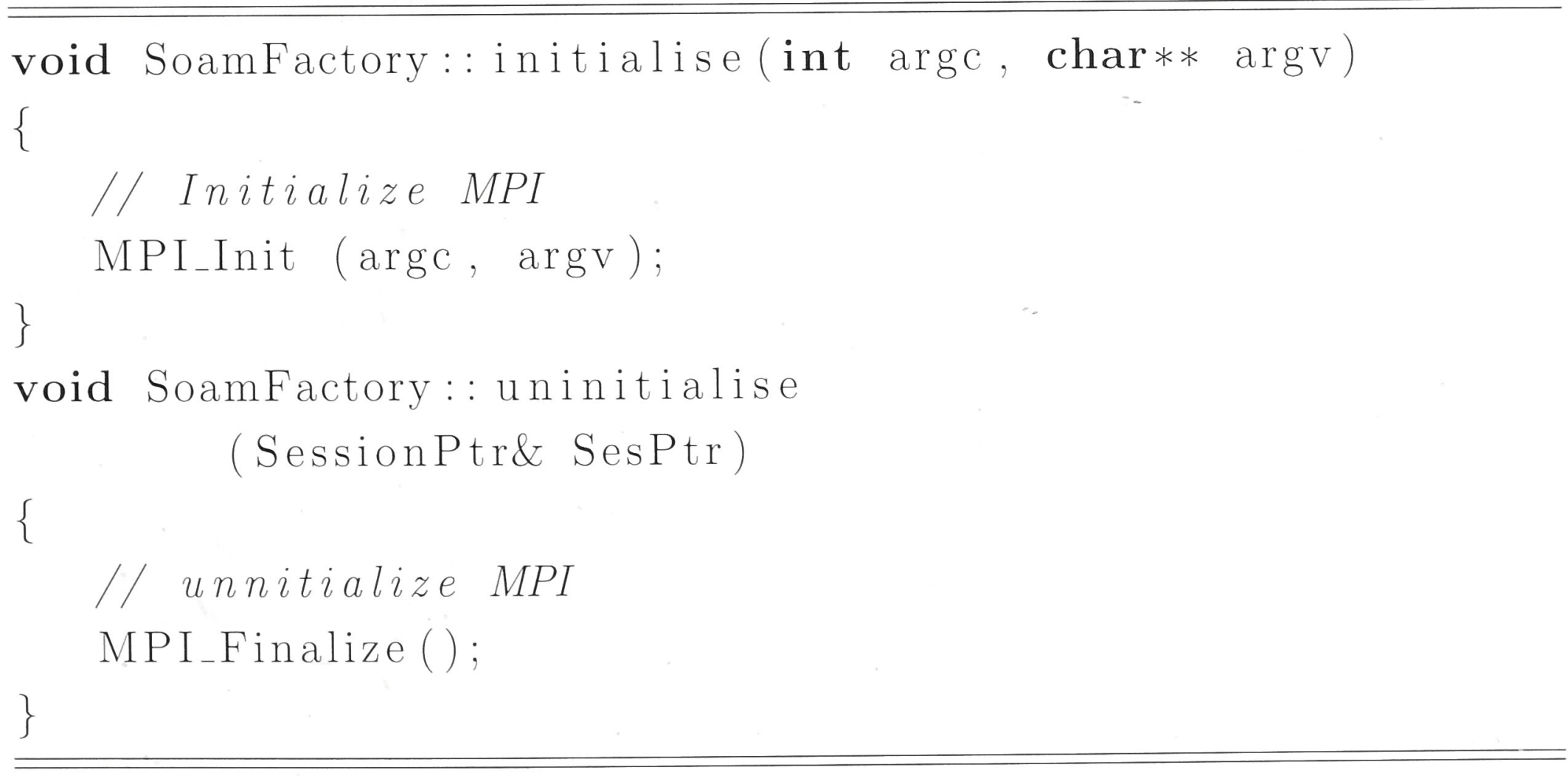

\section{B.1.1 Session Creation}

Listing B.2: Create Session \& Send Common Data

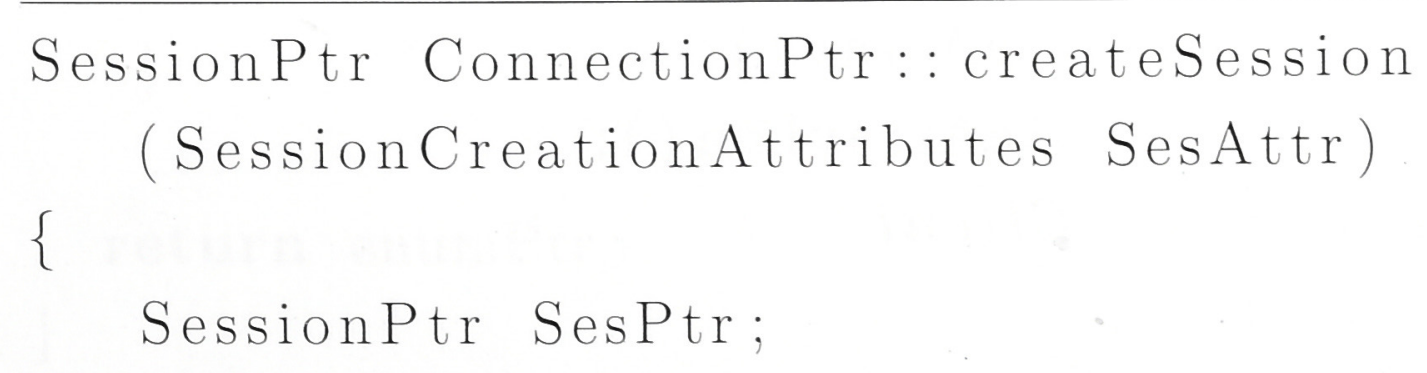




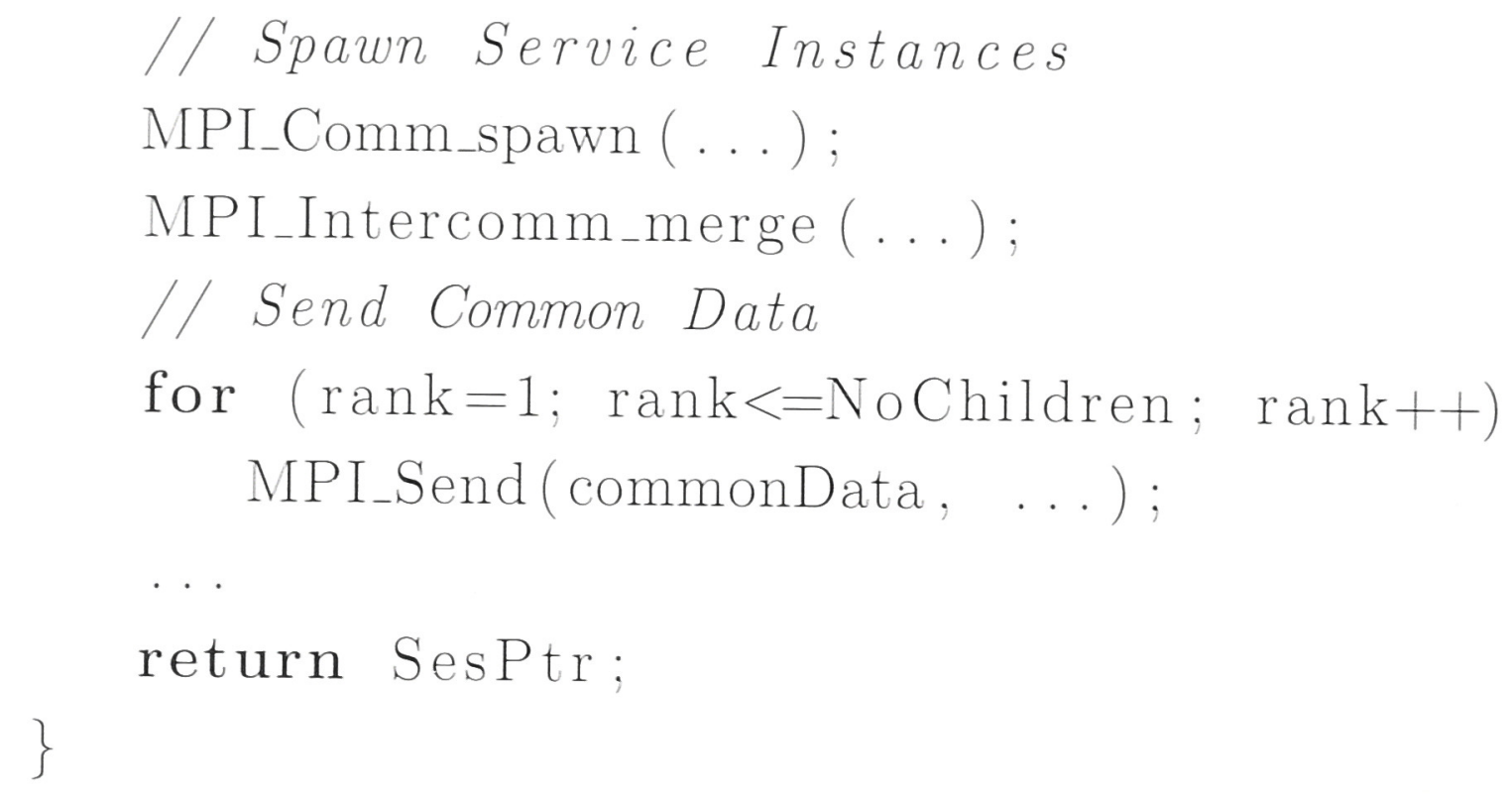

\section{B.1.2 Message Creation}

Listing B.3: Serialization \& (De-serialization

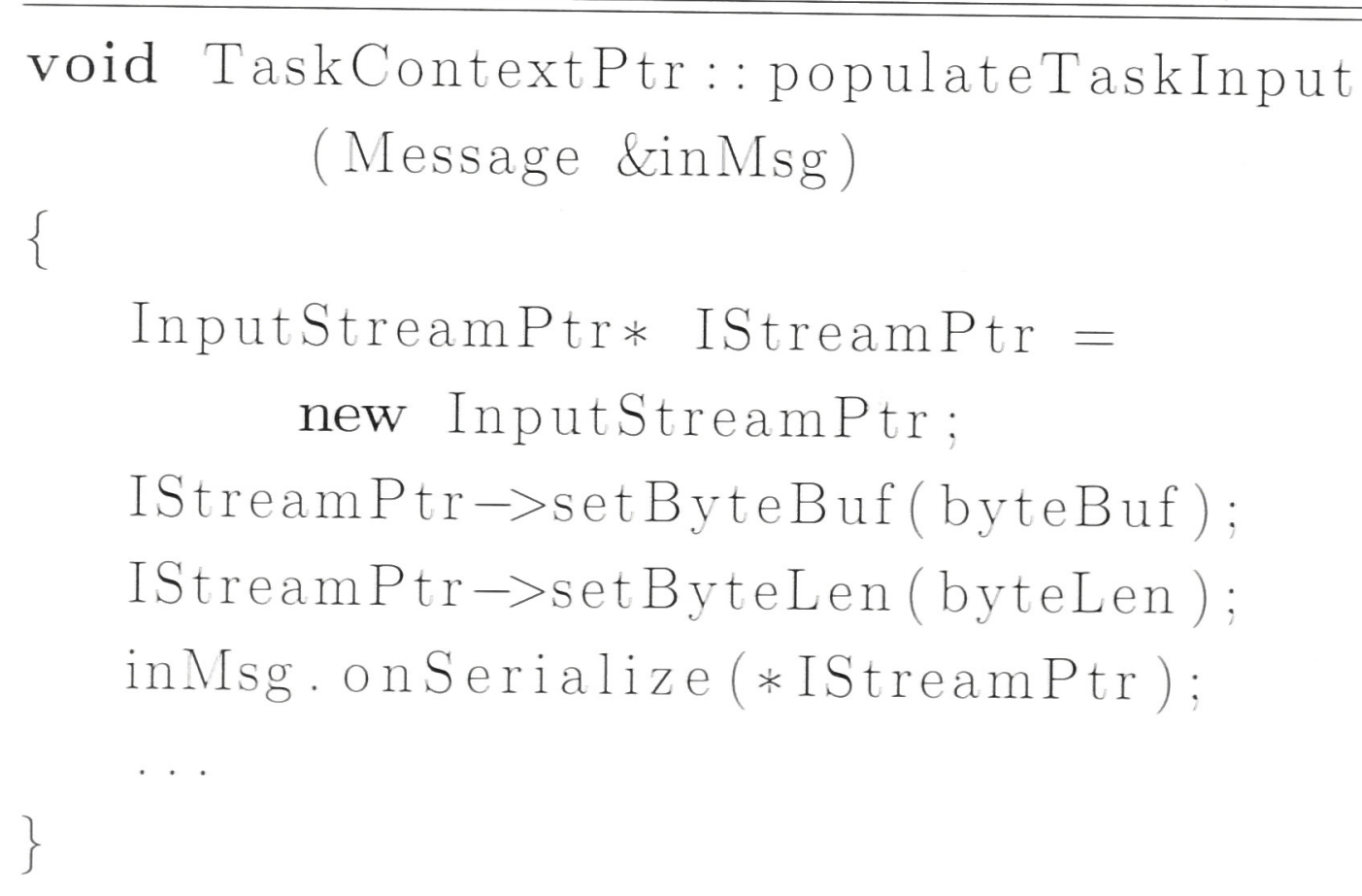


\}

\section{B.1.3 Task Communication}

Listing B.4: Task Creation

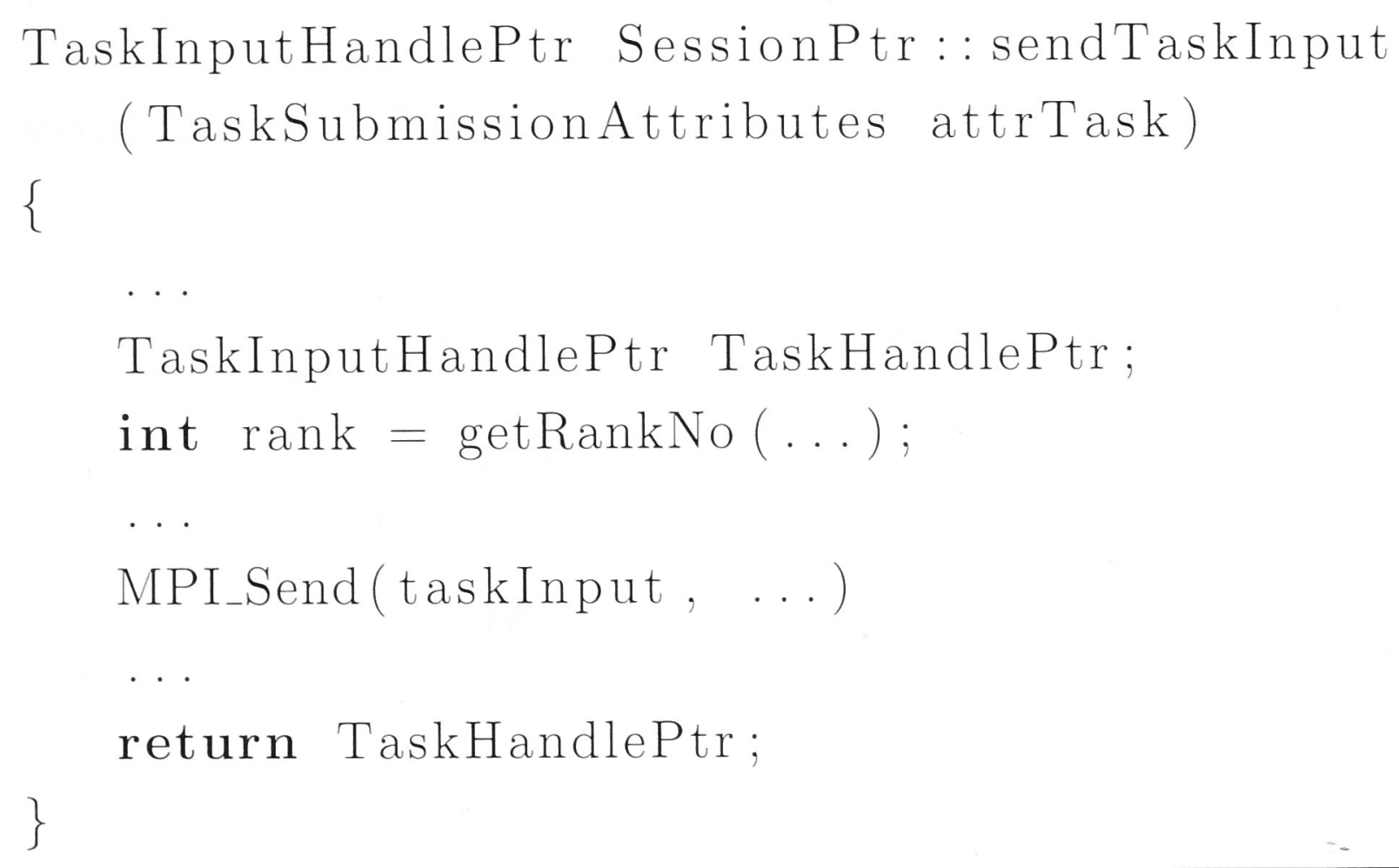

Listing B.5: Task Results

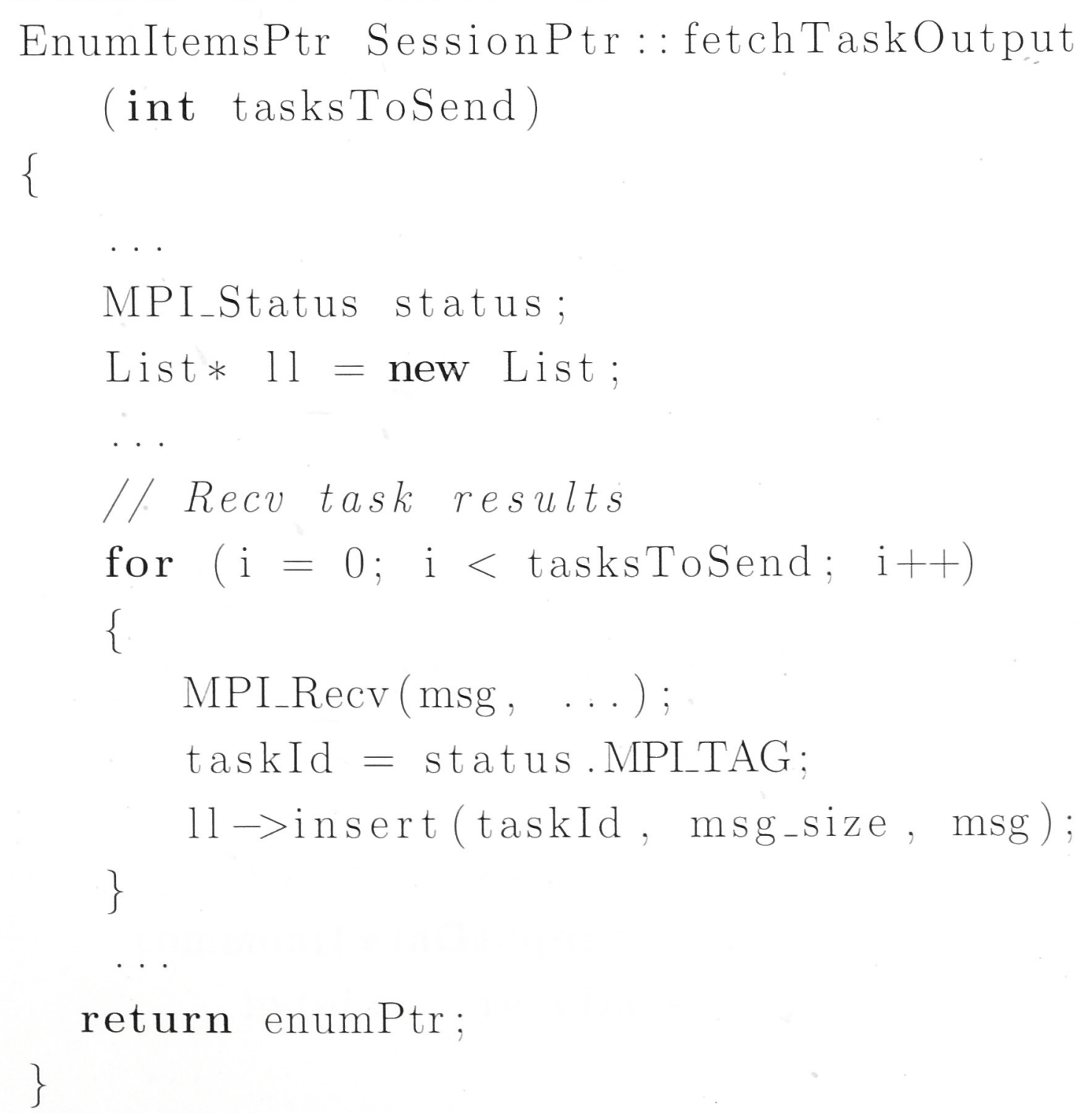




\section{B.2 Service Module}

Listing B.6: Common Data \& Task Management at Service

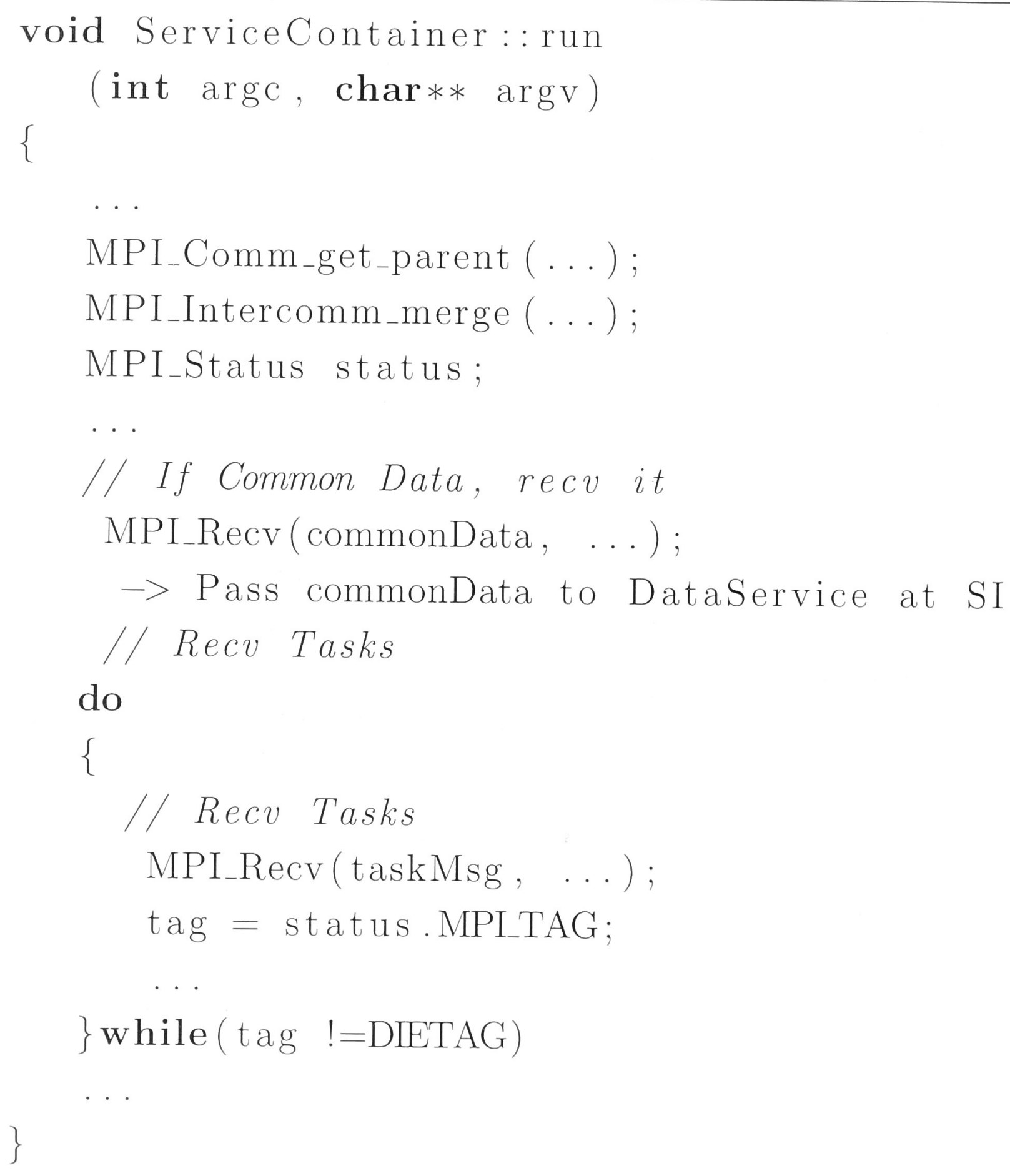

\section{B.3 Data Service Module}

Listing B.7: Common Data Functions

\section{void SessionContestPtr : : addCommonData}

commonDataList $\rightarrow$ insert (name, ..., inputData); 


\section{void SesionContestPtr: : getCommonData}

\{

commonDataList $\rightarrow$ getCommonData (name, ..., outputDataPtr);

\}

void SessionContestPtr: : putCommonData

\{

$\cdots$

dataUpdateList $\rightarrow$ insert (name, startPtr, len, data,...);

\}

void SessionContestPtr : : syncCommonData (name)

\{

.

// Get info (no of updates \& their length)...

// of all updates for 'name', in the SI

do (till finish) \{

bufSize=+dataUpdateList $\rightarrow$ get BufSize ();

noOfupdates ++ ;

\}

. .

// Share no of updates 6 length of updates per SI,

I/ among all SIs

MPI_Allgather (myUpdateLen, ... displacement , ...);

Share updates among all SIs with metadata

MPI_Allgatherv(myUpdateData, byteLen, MPI_BYTE,

recvData, recvDataLen, displacements, ...);

$\cdots$

// update common data list

commonDataList $\longrightarrow$ updateNodeBuf(name, startBytePtr, byteLen, recvData);

$1 /$ delete all synced updates from list 
dataUpdateList $\rightarrow$ deleteNameList (name);

// send updated CommonData to the client Data Service // (for iSync this step is omitted)

MPI_Send (sendBuf, ... , rankClient, ...) ;

\} 
Appendix C

Algorithms 


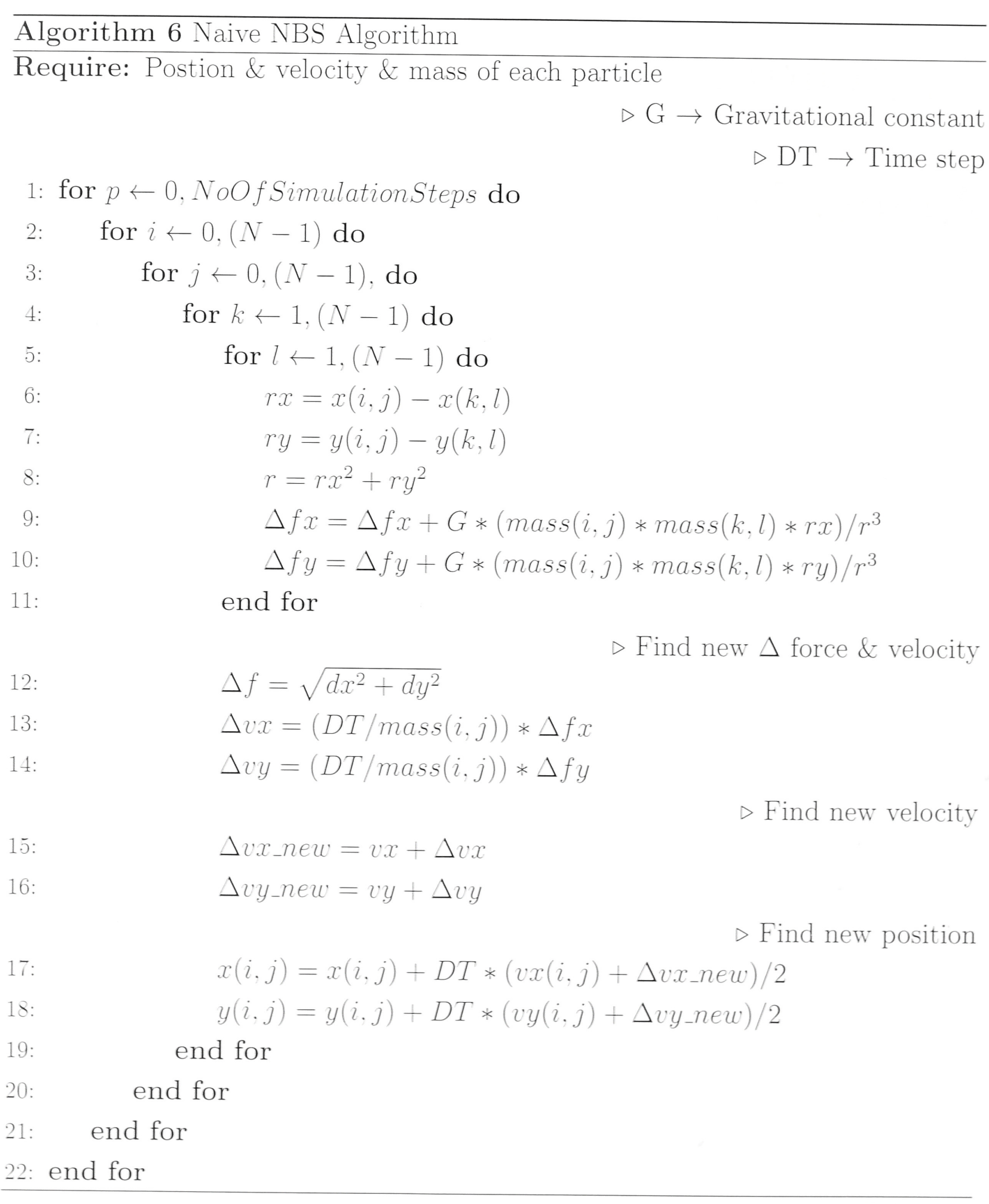



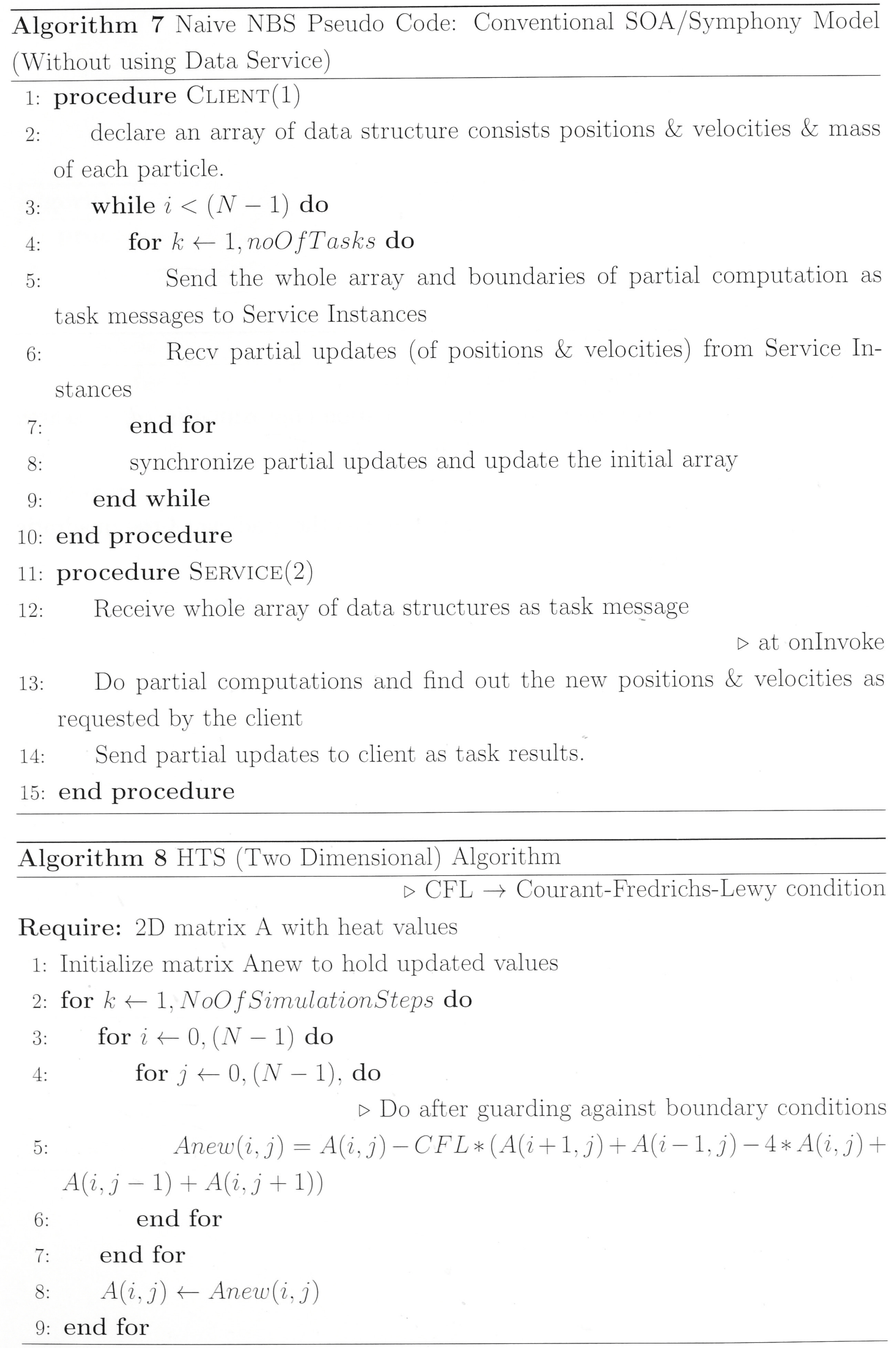


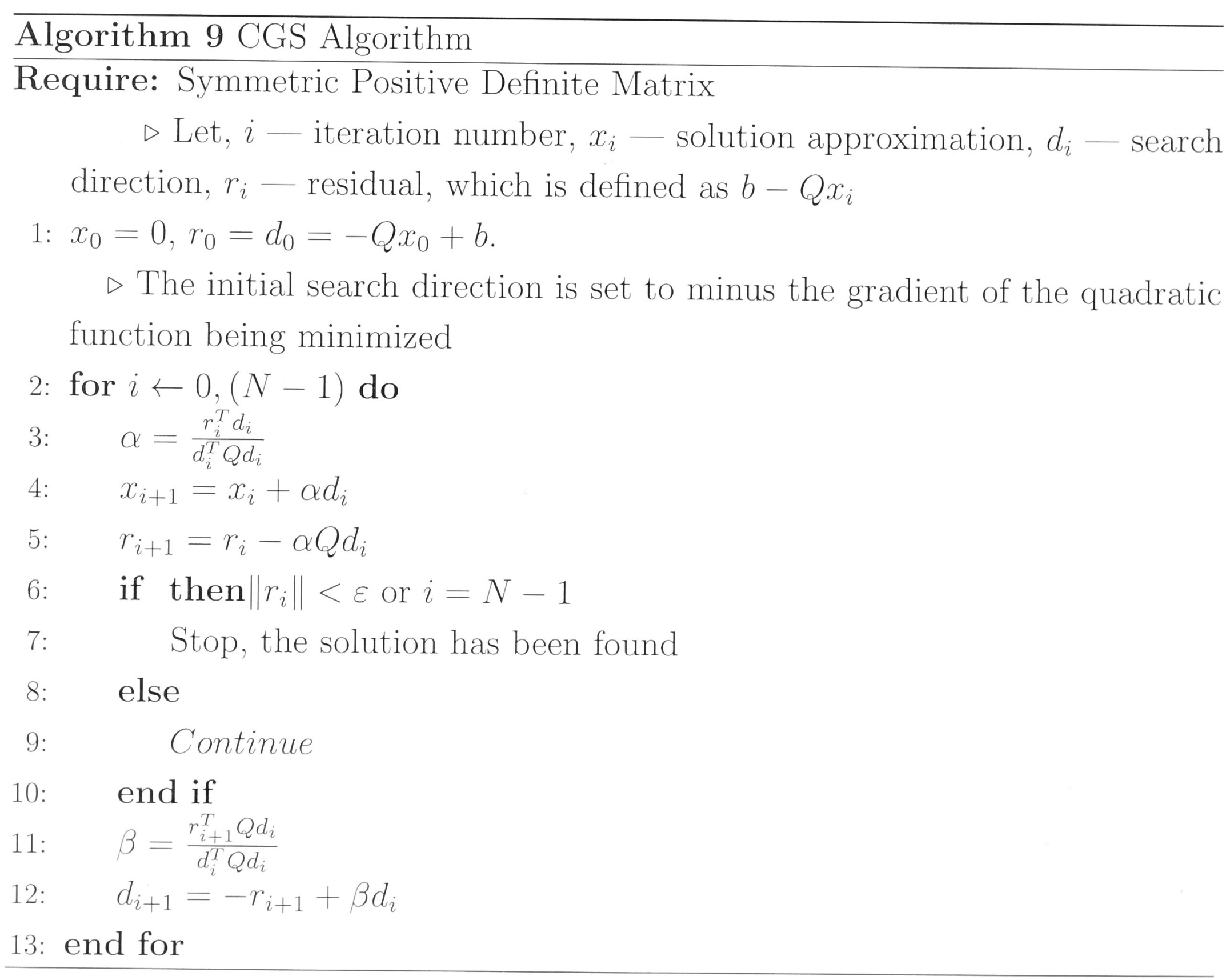




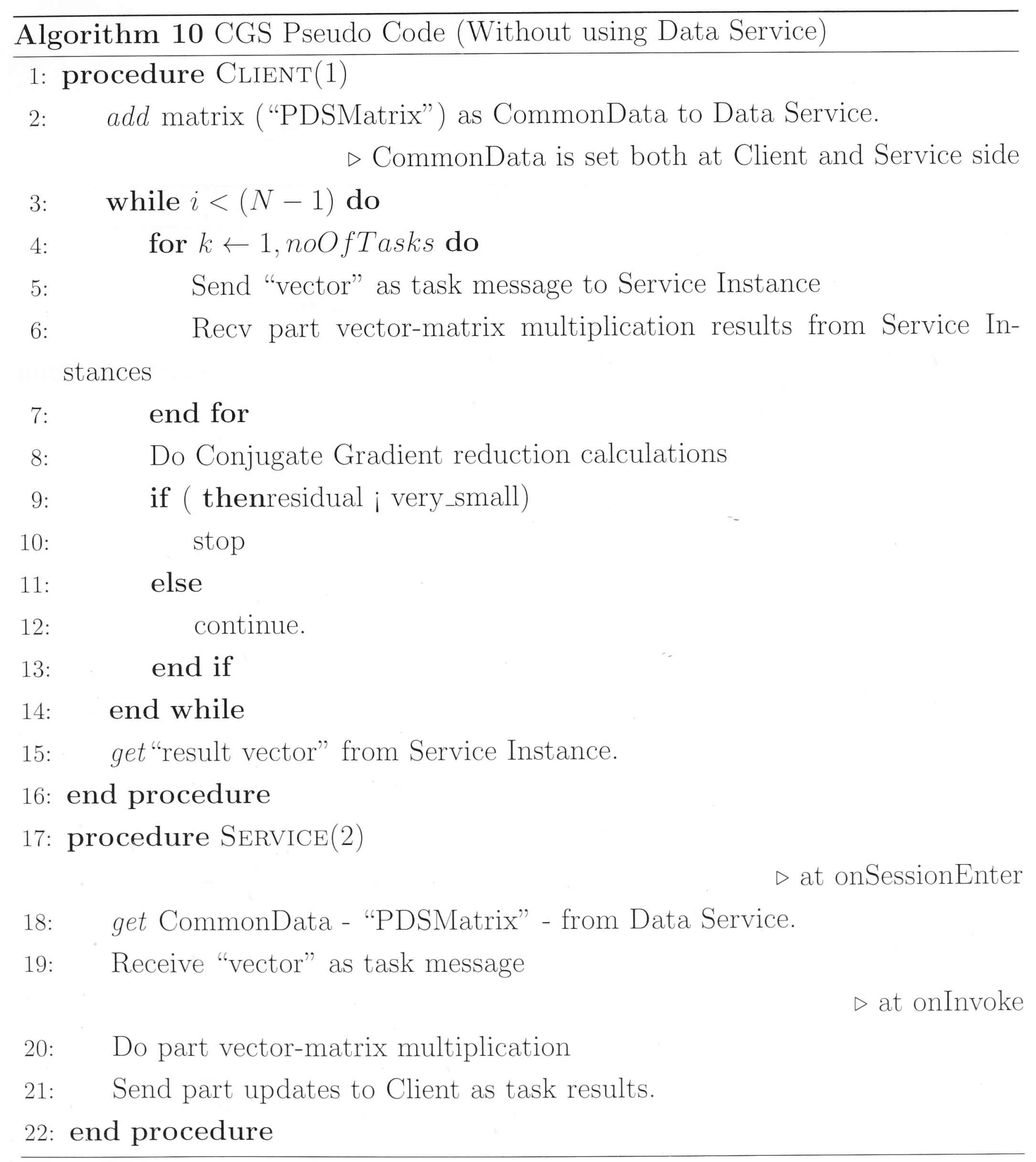




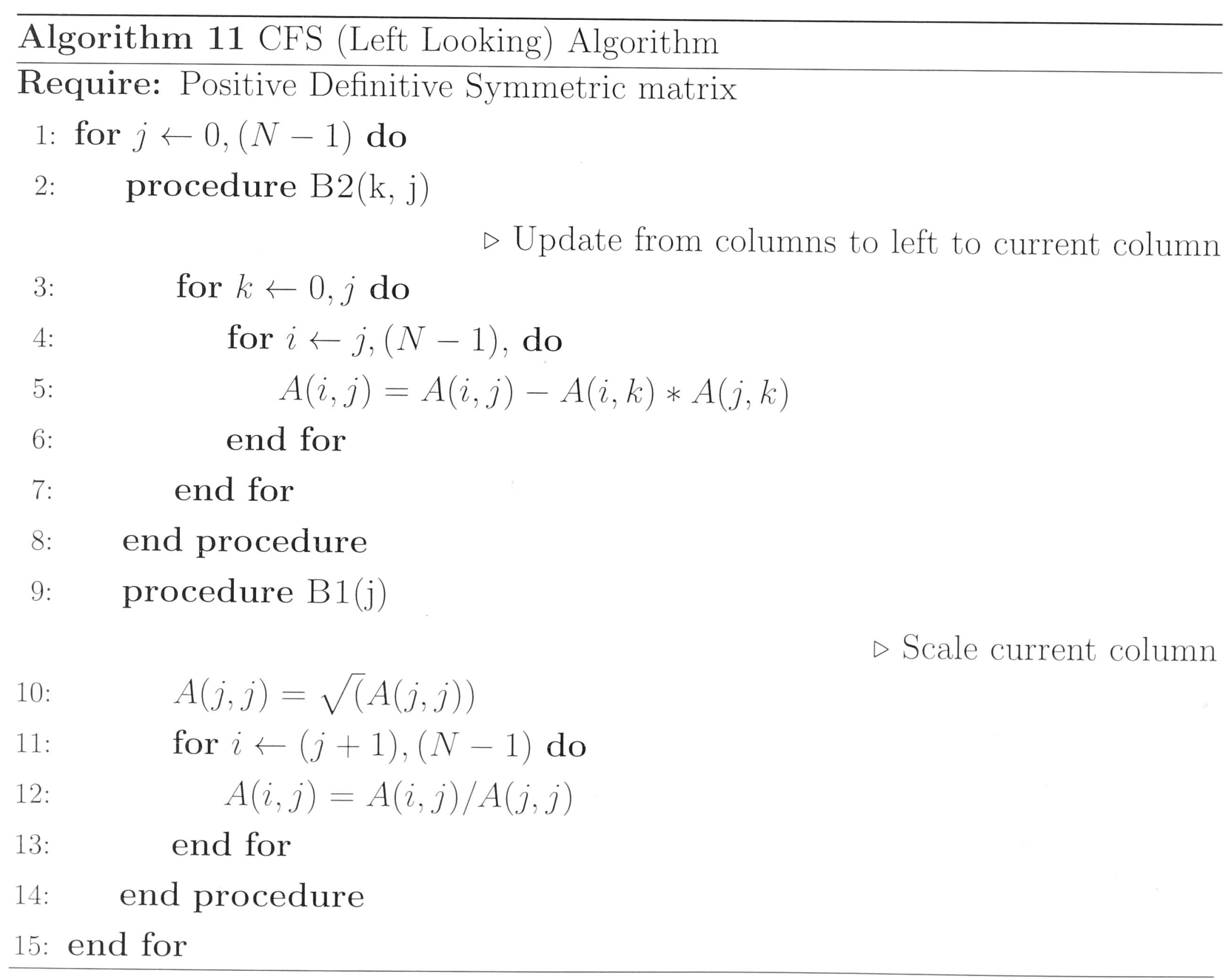

